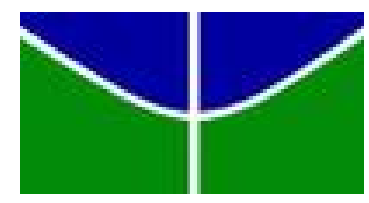

Universidade de Brasília

IE - Instituto de Exatas

Departamento de Estatística

\title{
Distribuição de Funções de Variáveis Aleatórias Dependentes e R-Vines Cópulas
}

Yuri Sampaio Maluf 

Yuri Sampaio Maluf

\section{Distribuição de Funções de Variáveis Aleatórias Dependentes e R-Vines Cópulas}

Dissertação de Mestrado apresentada ao Programa de Pós-Graduação em Estatística da Universidade de Brasília - UnB, como requisito parcial para a obtenção do Título de Mestre em Estatística.

Orientadora: Dra. Cira Etheowalda Guevara Otiniano.

Brasília

2015 

Maluf, Yuri S.

Distribuição de Funções de Variáveis Aleatórias Dependentes e R-Vines Cópulas.

159 páginas

Dissertação - Instituto de Exatas da Universidade de Brasília. Departamento de Estatística.

1. Distribuição de Soma

2. Distribuição de Produto

3. Distribuição de Razão

4. C e D-Vines Cópulas

5. Value-at-Risk

I. Universidade de Brasília. Instituto de Exatas. Departamento de Estatística. 



\section{Agradecimentos}

Agradeço a minha professora e orientadora, Cira Etheowalda, pela atenção e pela imensa ajuda e conhecimentos transmitidos, sem os quais não seria possível a construção deste trabalho. A toda equipe de professores da estatística. Adicionalmente ao professor e amigo Abimael por sua ajuda. A CAPES pelo suporte financeiro. A toda minha família, Ana Helena, minha mentora na Terra, Fares, Ana Júlia, Ana Cristhina, Berti, Andréa, Lindinalva, Bandit, Sivuca, e ao meu grande amor, Denise. E finalmente ao meu mentor espiritual. 


\section{Resumo}

Neste trabalho, estudamos a formulação da distribuição de funções de variáveis aleatórias contínuas dependentes. O mecanismo de modelagem da dependência é feita via funções cópulas. Dentre os resultados obtidos formulamos a expressão geral da distribuição da soma de $n$ variáveis aleatórias dependentes. Expandimos a abordagem para a distribuição de outras funções de variáveis aleatórias tais como o quociente, produto e uma combinação convexa. Por meio das R-Vines Cópulas, obtivermos também a expressão da soma de $n$ variáveis aleatórias em que cada componente é governada por um processo $\operatorname{GARCH}(\mathrm{p}, \mathrm{q})$. A partir deste resultado, calculamos o Value-at-Risk (VaR) e Expected Shortfalls (ES) da soma dessas variáveis. Em função desta estrutura, as medidas de risco passam a adquirir um comportamento dinâmico. Ao final do trabalho exibimos algumas ilustrações numéricas via simulação de Monte Carlo. Apresentamos também uma aplicação com dados reais provenientes de bolsas de valores da América Latina.

Palavras-chave: Distribuição da soma, produto e quociente de variáveis aleatórias; Cópulas; R-Vines; VaR; ES. 


\begin{abstract}
In this thesis, we studied the distribution of function of dependents continuous random variables. The modeling dependencies structures are made via copula functions. We obtain the general expression of the distribution of the sum of $n$ dependents random variables. This approach is expanded for other functions such as ratio, product and a convex combination. Using R-Vines Copulas, we also derive an expression of the sum of $n$ dependents random variables, being each component governed by $\operatorname{AR}(\mathrm{b})-\mathrm{GARCH}(\mathrm{p}, \mathrm{q})$ process. From these results, we assess the Value-at-Risk (VaR) and Expected Shortfalls (ES) of the sum of these variables. According to this structure, the VaR takes a dynamic behavior. At the end of this thesis, we show some numerical illustrations via Monte Carlo simulation. An application with real data from Latin American stock markets is also presented.
\end{abstract}

Keywords: Distribution of sum, product and quotient of random variables; Copulas; R-Vines; VaR; ES. 



\section{Lista de Figuras}

1.1 Gráfico da cópula $\Pi$. . . . . . . . . . . . . . . . . . . . 34

1.2 Gráfico da cópula $C_{U} \ldots \ldots \ldots$. . . . . . . . . . . . . . . 34

1.3 Gráfico da cópula $C_{L} \ldots \ldots \ldots \ldots \ldots$

1.4 Curvas de nível - cópula Gaussiana $\rho=0,6 \ldots . \ldots .38$

1.5 Curvas de nível - Cópula-t $\rho=0,6$ e $\nu=3 \ldots \ldots$. . . . . . 42

1.6 Dispersão $c_{\rho_{i}}^{G a}\left(F\left(x_{1}\right), F\left(x_{2}\right)\right), i=1,2,3$, marginal normal padrão . . . 43

1.7 Dispersão $c_{\rho_{i}, \nu}^{t}\left(F\left(x_{1}\right), F\left(x_{2}\right)\right), i=1,2,3$, marginal normal padrão $\ldots . \quad 44$

1.8 Clayton $c_{\theta_{i}}\left(F\left(x_{1}\right), F\left(x_{2}\right)\right), i=1,2,3$, marginal normal padrão . . . . 52

1.9 Joe $c_{\theta_{i}}\left(F\left(x_{1}\right), F\left(x_{2}\right)\right), i=1,2,3$, marginal normal padrão $\ldots . . .52$

1.10 Frank $c_{\theta_{i}}\left(F\left(x_{1}\right), F\left(x_{2}\right)\right), i=1,2,3$, marginal normal padrão . . . . . . 52

1.11 Gumbel $c_{\theta_{i}}\left(F\left(x_{1}\right), F\left(x_{2}\right)\right), i=1,2,3$, marginal normal padrão $\quad \ldots . \quad 53$

1.12 Ali-Mikhail-Haq $c_{\theta_{i}}\left(F\left(x_{1}\right), F\left(x_{2}\right)\right), i=1,2,3$, marginal normal padrão 53

1.13 Tawn $c_{\theta_{i}, \alpha, \beta}\left(F\left(x_{1}\right), F\left(x_{2}\right)\right), i=1,2,3$, marginal normal padrão $\quad \ldots . \quad 59$

1.14 Husler-Reiss $c_{\theta_{i}}\left(F\left(x_{1}\right), F\left(x_{2}\right)\right), i=1,2,3$, marginal normal padrão . . 60

1.15 Galambos $c_{\theta_{i}}\left(F\left(x_{1}\right), F\left(x_{2}\right)\right), i=1,2,3$, marginal normal padrão . . . 60 
1.16 Galambos (vermelho), Husler-Reiss (lilás), Tawn (verde) e Gumbel

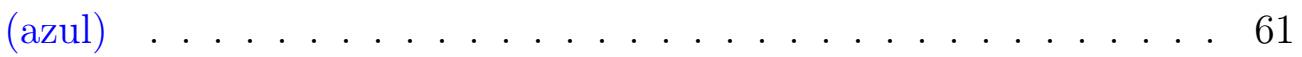

1.17 Mistura: $w \times \operatorname{Gumbel}(\theta=2)+(1-w) \times \operatorname{Frank}(\theta=-4,5) \ldots . . .65$

1.18 Mistura: $w \times \operatorname{Joe}(\theta=2)+(1-w) \times \operatorname{Clayton}(\theta=2,3) \ldots . . . .66$

2.1 Diagrama R-Vine para Drawable (D) e Canonical (C) Vines cópulas com $n=5 \ldots \ldots \ldots \ldots \ldots \ldots \ldots \ldots$

2.2 Diagrama D-Vine $m=3 \ldots \ldots \ldots \ldots$

2.3 Exemplo diagrama D-Vine $m=3 \ldots \ldots$. . . . . . . . . . 75

3.1 C-Convolução de uma Clayton, $\operatorname{com} \theta=0.5, \lambda_{F}=\lambda_{G}=2$ (preto) e distribuição Gama $W \sim \Gamma(2,2)$ (azul) . . . . . . . . . . . . . . 83

3.2 Convolução- $C$ de uma Clayton, $\operatorname{com} \theta=2, \lambda_{F}=2$ e $\lambda_{G}=2$ (preto) e distribuição Gama $W \sim \Gamma(2,2)$ (azul) . . . . . . . . . . . . . . . 84

6.1 Clayton: curvas de nível com marginais t-Student . . . . . . . . . . 116

6.2 Clayton: Value-at-Risk 1\% (tracejada) e 5\% (contínua). . . . . . . . 117

6.3 Clayton: Value-at-Risk 1\% (tracejada) e 5\% (contínua). . . . . . . . 118

6.4 Curva Sharpe Ratio. . . . . . . . . . . . . . . . . . . . . . . 119

6.5 Realização do processo $\operatorname{GARCH}(1,1)$ para $X_{1 t}$ e $X_{2 t}$. . . . . . . 120

6.6 Value-at-Risk $\operatorname{VaR}_{\alpha}\left(X_{1 t}+X_{2 t}\right) \ldots \ldots \ldots \ldots$

6.7 Diagramas D-Vine e C-Vine $n=3 \ldots \ldots$. . . . . . . . . 123

6.8 D-Vine: $F_{W_{3}}(t)$ Value-at-Risk $1 \%$ (tracejada) e 5\% (contínua). . . . 126

6.9 Realização do processo $\operatorname{GARCH}(1,1)$ para $X_{1 t}, X_{2 t}$ e $X_{3 t} \ldots \ldots 127$ 
6.10 Value-at-Risk: $\operatorname{VaR}_{\alpha}\left(X_{1 t}+X_{2 t}+X_{3 t}\right) \alpha=5 \%$ (contínua) $\alpha=1 \%$ (tracejada) . . . . . . . . . . . . . . . . . . . 129

6.11 Curvas de níveis das conjuntas $X_{1}$ e $X_{2}$ com marginais Gama . . . 132

6.12 Distribuição da razão $Y=\frac{X_{1}}{X_{1}+X_{2}}$ - cópula (azul) e independente (vermelho) . . . . . . . . . . . . . . . . . 133

6.13 Série histórica índices Merval (azul), Ibovespa (verde) e IPSA (vermelho) . . . . . . . . . . . . . . . . . . . 134

6.14 Série retornos dos índices. . . . . . . . . . . . . . . . . . 135

6.15 Diagrama D-Vine cópula . . . . . . . . . . . . . . . 136

6.16 Densidade t-Student, Normal (roxo) e estimador Kernel (preto). . . 137

6.17 Histograma $\hat{u}_{i}=F_{X_{i}}\left(x_{i}: \hat{\mu}, \hat{\sigma}, \hat{\nu}\right) \ldots \ldots . \ldots \ldots 138$

6.18 Matriz de dispersão e cópula empírica dos retornos dos índices . . . 138

6.19 Função $\lambda$ : teórico (cinza) empírico (preto) . . . . . . . . . . . . . 140

6.20 Value-at-Risk: $\operatorname{VaR}_{\alpha}\left(X_{1}+X_{2}+X_{3}\right) 1 \%$ (tracejada) $5 \%$ (contínua). 141

$6.21 \operatorname{VaR}_{\alpha}\left(X_{1 t}+X_{2 t}+X_{3 t}\right) \alpha=5 \%$ e variância condicionais dos índices 143

22 Função autocorrelação dos retornos . . . . . . . . . . . . . . . . . . 158

23 Função autocorrelação dos resíduos AR(1)-GARCH(1,1) . . . . . 159 



\section{Lista de Tabelas}

1.1 Algumas famílias de cópulas Arquimedianas . . . . . . . . . . . . 51

6.1 Parâmetros distribuição marginal $X_{1}$ e $X_{2}$. . . . . . . . . . . 115

6.2 Parâmetros cópula Clayton . . . . . . . . . . . . . . . . . 116

6.3 Estimação parâmetros: cópula Clayton e marginais t-Student. . . . 117

6.4 Medidas de risco de $X_{1}+X_{2} \ldots \ldots \ldots$. . . . . . . . . 118

6.5 Medidas de risco de $w X_{1}+(1-w) X_{2} \ldots \ldots . \ldots 118$

6.6 Parâmetros: distribuição marginal $X_{1}$ e $X_{2}$. . . . . . . . . . . . . . 119

6.7 Estimação parâmetros: cópula Clayton e GARCH com inovações t-Student. . . . . . . . . . . . . . . . . . 121

6.8 Parâmetros: D-Vine e C-Vine cópula $n=3 . \quad$. . . . . . . . . . 123

6.9 Parâmetros distribuição marginal. . . . . . . . . . . . . . . . . . . 124

6.10 Estimação parâmetros: D-Vine cópula e marginais t-Student. . . . . 124

6.11 Estimação parâmetros: C-Vine cópula e marginais t-Student. . . . . 125

6.12 Medidas de risco de $W_{3} \ldots \ldots \ldots$. . . . . . . . . . 126

6.13 Parâmetros: distribuição marginal $X_{1}, X_{2}$ e $X_{3}$. . . . . . . . 126 
6.14 Estimação parâmetros: cópula D-Vine e GARCH com inovações t-Student. . . . . . . . . . . . . . . . . . . 128

6.15 Parâmetros distribuição Gama. . . . . . . . . . . . . . . . . 131

6.16 Parâmetros Cópula-t. . . . . . . . . . . . . . . . . . . 131

6.17 Estimação: Cópula-t e marginais Gama. . . . . . . . . . . . . . . 133

6.18 AIC e BIC marginais . . . . . . . . . . . . . . . . 137

6.19 Parâmetros estimado distribuição t-Student . . . . . . . . . . . . 137

6.20 Seleção cópulas baseada no teste de Vuong . . . . . . . . . . . . . 139

6.21 Estimação parâmetros cópulas . . . . . . . . . . . . . . . . . . . 140

6.22 Medidas de risco de $W_{3} \ldots \ldots \ldots$. . . . . . . . . . . . . 141

6.23 Estimação parâmetros marginais $X_{1}, X_{2}$ e $X_{3}$. . . . . . . . . 142

6.24 Estimação parâmetros cópulas . . . . . . . . . . . . . . . . . . . . . 142

25 Teste estacionaridade ADF dos retornos . . . . . . . . . . . . 158

26 Teste de autocorrelação Ljung-Box retornos . . . . . . . . . . . . . 158

27 Teste de autocorrelação Ljung-Box resíduos AR(1)-GARCH(1,1) . 159 


\section{Sumário}

$\begin{array}{ll}\text { Introdução } & 1\end{array}$

1 Cópulas e Dependência $\quad 7$

1.1 Distribuição Multivariada . . . . . . . . . . . . . . . . 7

1.2 Funções Cópulas . . . . . . . . . . . . . . . . . . . 11

1.3 Algumas Propriedades de Cópulas . . . . . . . . . . . . . . . . . 16

1.3.1 Cópula Sobrevivência . . . . . . . . . . . . . . . . 16

1.3.2 Cópula Condicional . . . . . . . . . . . . . . . . . . 20

1.4 Medidas de Dependência . . . . . . . . . . . . . . . . 24

1.5 Principais Famílias de Cópulas Bivariadas . . . . . . . . . . . 33

1.5.1 Cópulas Elementares . . . . . . . . . . . . . . 33

1.5.2 Cópulas Elípticas . . . . . . . . . . . . . . . . . 35

1.5.3 Cópulas Arquimedianas . . . . . . . . . . . . . . . . . 44

1.5.4 Cópulas de Valores Extremos . . . . . . . . . . . . . . . 54

1.6 Misturas de Cópulas . . . . . . . . . . . . . . . . . . . . 62

2 R-Vines Cópulas $\quad 67$ 
2.1 Distribuição Multivariada por Pares de Cópulas . . . . . . . . . . . 68

2.2 Vine Cópulas . . . . . . . . . . . . . . . . . . . 73

3 Distribuição de Funções de Variáveis Aleatórias via Funções Có$\begin{array}{ll}\text { pulas } & 77\end{array}$

3.1 Convolução via Cópulas . . . . . . . . . . . . . . . . . 78

3.2 Distribuição do produto e do quociente . . . . . . . . . . . . . 91

4 Distribuição da Soma com Modelos AR-GARCH 97

5 Estimação de Cópulas $\quad 107$

5.1 Máxima Verossimilhança Exato . . . . . . . . . . . . . . . . 108

5.2 Máxima Verossimilhança em Dois Passos . . . . . . . . . . . . 111

6 Ilustrações Numéricas

6.1 Exemplos por Simulação de Monte Carlo . . . . . . . . . . . . . . . 114

6.1.1 Caso Bivariado .................. 115

6.1.2 Caso Multivariado .................. . . 122

6.1 .3 Distribuição da Razão . . . . . . . . . . . . . . . . . . . 130

6.2 Aplicação com Dados Reais . . . . . . . . . . . . . . . . . 134

Considerações Finais 
Apêndice B 



\section{Introdução}

A distribuição de funções de variáveis aleatórias é utilizada frequentemente em muitas áreas da estatística. A importância destas distribuições surge em razão da grande aplicabilidade em diversos ramos da estatística. Na inferência estatística, por exemplo, as distribuições Qui-Quadrado, Gama e Estáveis originam-se da soma $X_{1}+\cdots+X_{n}$ das $X_{i}$ 's variáveis aleatórias independentes. Já as distribuições t-Student, F de Snedecor, Slash, Beta e Dirichlet são provenientes de quocientes de variáveis aleatórias independentes.

Em diversas aplicações nas áreas de finanças, atuária, hidrologia, engenharia de confiabilidade, análise de sobrevivência, entre outras, aparecem com recorrência também as distribuições de funções do tipo soma $\sum_{i=1}^{n} X_{i}$, combinação convexa $\sum_{i=1}^{n} \pi_{i} X_{i}$, produto $X_{1} X_{2}$, quocientes $X_{1} / X_{2}$ e $X_{1} /\left(X_{1}+X_{2}\right)$, aqui denominados de quociente ou razão do tipo 1 e tipo 2, respectivamente. Sob suposição de independência, muitos estudos na literatura tratam dessas funções de variáveis aleatórias com as mais diversas distribuições. Nadarajah e Dey (2006) analisaram a distribuição do módulo do produto e do quociente do tipo 1 de variáveis aleatórias t-Student. Idrizi (2014) estudou a distribuição do produto e do quociente tipo 1 de variáveis aleatórias Pareto e Kumaraswamy. Com a distribuição Beta, PhamGia (2000) formulou a distribuição da razão do tipo 1 e 2 e com esses resultados aplicou na área de engenharia de confiabilidade. Shakil (2006) deriva a distribuição da razão do tipo 1 de variáveis aleatórias gamas. Já Ali et al. (2007) obtiveram a 
distribuição e os momentos do quociente tipo 2 entre Gamas invertidas em termos de funções especiais.

No entanto, poucos são os estudos para a distribuição das funções acima citadas, quando as variáveis aleatórias não são independentes. Dentre alguns deles, podemos destacar Press (1969) que tratou do quociente do tipo 1 de t-Student bivariada motivado por aplicações em econometria bayesiana. A partir da distribuição Beta bivariada, proposta por Libby e Novick (1982), Nagar et al. (2009) formularam a distribuição do produto e do quociente tipo 1 e 2 em termos da função hipergeométrica de Gauss. Com a distribuição Lomax bivariada, Nadarajah (2005b) obteve a distribuição da soma, produto e razão do tipo 2 em termos da função hipergeométrica de Gauss. Essas mesmas distribuições foram derivadas por Nadarajah (2005a) deriva a distribuição a partir da Gumbel bivariada. Vale a pena notar que em todos esses as estruturas de dependência adotadas são distribuições bivariadas específicas.

A obtenção de uma distribuição multivariada apropriada a um modelo probabilístico pode ser de difícil determinação. Uma alternativa para sua construção é através de função de cópulas. A vantagem de sua utilização reside na segregação das distribuições marginais com a função de dependência. Em função disso, as cópulas disponibilizam uma gama de estruturas de dependência, o que permite uma elaboração bem flexível de distribuições multivariadas. A teoria de cópulas iniciou-se com Sklar (1973) sendo extensivamente estudada por outros pesquisadores. Um livro clássico de teoria de cópulas, citado recorrentemente na literatura, é o do Nelsen (2006).

Apesar da ampla variedade de famílias de funções cópulas, elas se restringem majoritariamente a cópulas bivariadas. As cópulas multivariadas são, em geral, determinadas por um único parâmetro, o que limita a estrutura de dependência mútua entre as variáveis. Uma alternativa para a construção de distribuições 
multivariadas é por meio de cópulas bivariadas conhecida por PCC (Pair Copulas Constructions). Este método tem origem no trabalho de Joe (1996), em que a distribuição $n$-dimensional é dada a partir das marginais e de $n(n-1) / 2$ parâmetros de dependência, um para cada par de marginais. Posteriormente, Bedford e Cooke (2002) introduziram uma estrutura gráfica chamada de Regular (R-) Vines Cópulas para organizar a decomposição entre as variáveis. A decomposição pode ser de duas especies: Drawable (D-) Vines ou Canonical (C-)Vines Cópulas.

As cópulas têm-se mostrado muito úteis em uma série de aplicações. Na área de finanças, em especial, Cherubini et al. (2004) tratam de uma ampla variedade de utilização de cópulas. Uma aplicação recorrente é a modelagem de dependência entre ativos. Um ponto interessante que surge a partir daí, é a mensuração do risco de um portfólio pelo Value-at-Risk de $W_{n}=X_{1}+\cdots+X_{n}$ com nível de $\alpha \times 100 \%$ de confiança dado por

$$
\operatorname{VaR}_{\alpha}\left(W_{n}\right)=-\sup \left\{w \in \mathbb{R} \mid P\left(W_{n} \leq w\right) \leq \alpha\right\}
$$

em que $W_{n}$ representa o retorno de uma carteira de investimento contendo $n$ ativos. Em síntese, o $\operatorname{VaR}_{\alpha}\left(W_{n}\right)$ capta o máximo das perdas potenciais que ocorrem com $\alpha \times 100 \%$ para um dado período. O Value-at-Risk se tornou muito popular, sobretudo em virtude do comitê de supervisão bancária de Basileia adotá-lo como medida padrão (Choudhry, 2006). Uma dificuldade é determinar a distribuição de $W_{n}$ levando-se em conta a dependência entre os ativos.

A formulação da distribuição da soma de duas variáveis aleatórias dependentes induzida por uma função cópula bivariada foi desenvolvida por Cherubini et al. (2011). A generalização desta formulação, para o caso de $n$ variáveis aleatórias, foi proposta por Irene e Klaus (2014). Estes autores sugerem ainda aplicações na área de finanças com o cálculo do Value-at-Risk. 
Neste trabalho estudamos a distribuição da soma, combinação convexa, produto e quociente tipo 1 e 2 de variáveis aleatórias, considerando uma estrutura de dependência dada por um função cópula. No que tange a distribuição da soma, estendemos a abordagem de Cherubini et al. (2011) para o caso $n$-dimensional, ampliando também para a combinação convexa. A expressão obtida é análoga a encontrada por Irene e Klaus (2014), porém com uma demonstração alternativa. Examinamos também o caso da distribuição da soma onde a função cópula é gerada a partir da mistura de outras cópulas, o que permite uma estrutura de dependência mais flexível. Outro ponto tratado neste trabalho foi a dedução das expressões gerais para a distribuição do produto e dos quocientes $X_{1} / X_{2}$ e $X_{1} /\left(X_{1}+X_{2}\right)$.

Motivado por aplicações na área de finanças, estendemos o estudo também para a distribuição da soma e da combinação convexa quando o vetor de variáveis aleatórias $\left(X_{1 t}, \ldots, X_{n t}\right)$ é governado por um processo $\mathrm{AR}(\mathrm{b})-\mathrm{GARCH}(\mathrm{p}, \mathrm{q})$, em que a distribuição multivariada das inovações de cada processo possui dependência dada por uma função cópula. A incorporação dessa característica confere um caráter dinâmico ao Value-at-Risk, pois para cada tempo $t$ obtém-se valores distintos do $\operatorname{VaR}_{\alpha, t}\left(W_{n t}\right)$.

Assim, no primeiro capítulo realizamos uma ampla revisão da teoria de Cópulas, incluindo as principais famílias de distribuições, medidas de dependência, cópula condicional, bem como as misturas de cópulas. No Capítulo 2 introduzimos o mecanismo de construção de distribuições multivariada via PCC e as classes das R-Vines cópulas, mais especificamente as C-Vine e D-Vine.

A partir dos elementos destacados nos capítulos anteriores, no Capítulo 3 apresentamos os principais resultados do trabalho seguidos de alguns exemplos. Na seção 3.1, tratamos da distribuição da soma e combinações convexas de variáveis aleatórias dependentes. Na Proposição 4 e no Corolário 7 exibimos a formulação da distribuição da soma e combinação convexa de $n$ variáveis aleatórias, 
respectivamente. Ainda na mesma seção, no Corolário 5, obtemos a distribuição da soma de duas variáveis aleatórias dependentes quando a função cópula é baseada na mistura de duas outras cópulas. Na seção 3.2, a Proposição 5 apresenta a expressão da distribuição do produto $X_{1} X_{2}$ e quociente $X_{1} / X_{2}$ e na Proposição 6 a expressão da distribuição do quociente $X_{1} /\left(X_{1}+X_{2}\right)$.

No Capítulo 4 exploramos o caso de variáveis aleatórias envolvendo processo $\operatorname{AR}(\mathrm{b})-\mathrm{GARCH}(\mathrm{p}, \mathrm{q})$. Na Proposição 10 enunciamos a formulação da distribuição da soma de variáveis aleatórias onde as inovações de cada processo AR(b)$\operatorname{GARCH}(p, q)$ possui dependência induzida por funções cópulas. Seguindo esta mesma estrutura, a Proposição 10 exibe a expressão para o caso da combinação convexa. No Capítulo 5 abordamos brevemente os métodos de estimação dos parâmetros das cópulas.

Por fim, no Capítulo 6 exibimos ilustrações numéricas a fim de analisar os resultados tratados nos capítulos anteriores. Na seção 6.1 construímos alguns exemplos via simulação de Monte Carlo. A partir das expressões enunciadas nos Capítulos 3 e 4 e dos parâmetros estimados, a partir dos dados gerados pela simulação, traçamos a distribuição da soma, combinação convexa com e sem estrutura GARCH, tanto para o caso bivariado quanto para o trivariado. Ao fim desta seção, realizamos o mesmo procedimento para a distribuição de $X_{1} /\left(X_{1}+X_{2}\right)$. Na seção 6.2 utilizamos dados reais referentes às séries históricas dos principais índices das bolsas de valores das três maiores economias da América Latina: Brasil, Argentina e Chile. A partir dos retornos dos índices, modelamos a distribuição multivariada via D-Vine cópula e construímos a distribuição da soma dos log's retornos e as respectivas medidas de risco $\operatorname{VaR}_{\alpha}\left(W_{3}\right)$. Na sequência, incluímos a característica heterocedástica dos log's retornos via $\mathrm{AR}(1)-\mathrm{GARCH}(1,1)$ e modelamos novamente a distribuição da soma. Ao final, geramos uma série histórica $\operatorname{dos} \operatorname{VaR}_{\alpha, t}\left(W_{n t}\right)$ ao longo do ano de 2015 . 



\section{Capítulo 1}

\section{Cópulas e Dependência}

Nas últimas décadas, o estudo e aplicabilidade das funções cópulas tem-se tomado bastante popular em diversas áreas da estatística aplicada devido à grande quantidade disponível de funções cópulas que podem ser utilizadas como funções de distribuição multivariadas.

Neste capítulo, inicialmente abordamos alguns aspectos importantes acerca das distribuições multivariada e das cópulas. Na sequência, será tratada as medidas de dependência, assim como as principais famílias de funções cópulas bivariadas e suas propriedades. Ao longo deste capítulo as definições e as propriedades inseridas são baseadas nos trabalhos de Nelsen (2006) e Embrechts et al. (2001a), bem como boa parte da notação seguida por estes autores.

\subsection{Distribuição Multivariada}

Um ponto primordial deste trabalho fundamenta-se a partir da ideia de distribuição multivariada. A definição da função de distribuição multivariada e suas propriedades foram retiradas de James (2011) e Embrechts et al. (2001a). Todavia, antes de definir uma função de distribuição multivariada introduziremos 
alguns conceitos preliminares que serão utilizados na seção subsequente.

Primeiro introduzimos o operador diferença $\Delta_{a_{k}}^{b_{k}}$ aplicado a uma função real $H: \mathbb{R}^{n} \rightarrow \mathbb{R}$ em um intervalo $I_{k}=\left(a_{k}, b_{k}\right] \operatorname{com} k=1, \ldots, n$ que é caracterizada pelas seguinte operação,

$$
\Delta_{a_{k}}^{b_{k}} H\left(x_{1}, \ldots, x_{n}\right)=H\left(x_{1}, \ldots, x_{k-1}, b_{k}, x_{k+1}, \ldots, x_{n}\right)-H\left(x_{1}, \ldots, x_{k-1}, a_{k}, x_{k+1}, \ldots, x_{n}\right)
$$

Utilizamos a notação usual $D o m H$ e $R a n H$ para o domínio e o contra-domínio da função $H$, respectivamente. Também denotamos o conjunto $S=[0,1]^{n} \subseteq \mathbb{R}^{n}$ referente ao um hipercubo unitário de $\mathbb{R}^{n}$ e dizemos que é definido quase certamente se o conjunto de pontos de $\mathbb{R}^{n}$ que não pertence a $S$ possui medida nula.

A partir do operador (1.1) definimos $V o l_{H}$.

Definição 1 Sejam $S_{1}, S_{2}, \ldots, S_{n}$ subconjuntos não vazios de $\mathbb{R}, H$ uma função real de $n$ variáveis tal que $D o m H=S_{1} \times S_{2} \times \cdots \times S_{n}$ e seja também $B=[\boldsymbol{a}, \boldsymbol{b}]=$ $\left(\left[a_{1}, b_{1}\right] \times\left[a_{2}, b_{2}\right] \times \ldots \times\left[a_{n}, b_{n}\right]\right)$ um cubo $n$-dimensional em que os vértices estão contidos no DomH onde $\boldsymbol{a} \leq \boldsymbol{b}$ para todo $a_{k} \leq b_{k}, k=1,2, \ldots, n$. Então o HVolume sobre o cubo $n$-dimensional, $B=[\boldsymbol{a}, \boldsymbol{b}]$, é dado pela aplicação sucessiva do operador diferença,

$$
V_{o l}(B)=\Delta_{\boldsymbol{a}}^{\boldsymbol{b}} H(\boldsymbol{x})=\Delta_{a_{1}}^{b_{1}} \Delta_{a_{2}}^{b_{2}} \cdots \Delta_{a_{n}}^{b_{n}} H\left(x_{1}, x_{2}, \ldots, x_{n}\right),
$$

em que os pontos onde são aplicados as diferenças são os vértices do cubo $n$ dimensional.

Temos também que a função $H$ é não decrescente na $k$-ésima componente se $\left(t_{1}, t_{2}, \ldots, t_{k-1}, x, t_{k+1}, \ldots, t_{n}\right)$ e $\left(t_{1}, t_{2}, \ldots, t_{k-1}, y, t_{k+1}, \ldots, t_{n}\right)$ pertencente ao Dom $H$, com $x \leq y$ então ocorre $H\left(t_{1}, t_{2}, \ldots, t_{k-1}, x, t_{k+1}, \ldots, t_{n}\right) \leq H\left(t_{1}, t_{2}, \ldots, t_{k-1}, y, t_{k+1}, \ldots, t_{n}\right)$.

A partir deste ponto temos os elementos para definir uma função de distri- 
buição multivariada.

Definição 2 (Distribuição Multivariada): Uma função $F: \mathbb{R}^{n} \longrightarrow[0,1]$ é uma distribuição multivariada se satisfaz a seguintes condições:

1. $F\left(x_{1}, x_{2}, \ldots, x_{n}\right)$ é não-decrescente em cada uma das variáveis.

2. $F\left(x_{1}, x_{2}, \ldots, x_{n}\right)$ é contínua a direita em cada uma das variáveis.

3. Para qualquer índice $k=1,2, \ldots, n$,

$$
\lim _{x_{k} \rightarrow-\infty} F\left(x_{1}, x_{2}, \ldots, x_{k-1}, x_{k}, x_{k+1}, \ldots, x_{n}\right)=0
$$

e também

$$
\lim _{\forall k, x_{k} \rightarrow+\infty} F\left(x_{1}, x_{2}, \ldots, x_{k-1}, x_{k}, x_{k+1}, \ldots, x_{n}\right)=1
$$

4. $\operatorname{Vol}_{F_{\boldsymbol{X}}}\left(S_{1}, S_{2}, \ldots, S_{n}\right) \geq 0$, em que $\forall S_{k}=\left(a_{k}, b_{k}\right] \subseteq \mathbb{R}$ em que $k=1,2, \ldots, n$.

A condição 3 garante que $0 \leq F\left(x_{1}, x_{2}, \ldots, x_{n}\right) \leq 1$.

Neste trabalho, denotaremos as funções marginais da distribuição multivariada $F_{\boldsymbol{X}}$ por $F_{1}, F_{2}, \ldots, F_{n}$ ou por $F_{X_{1}}, F_{X_{2}}, \ldots, F_{X_{n}}$. Portanto, dada uma distribuição multivariada $F_{\boldsymbol{X}}$ é possível obter:

1. a distribuição marginal $F_{k}$ tomando o limite $\forall x_{j \neq k} \rightarrow \infty$. Em outras palavras,

$$
F_{k}\left(x_{k}\right)=\lim _{\forall j \neq k, x_{j} \rightarrow \infty} F\left(x_{1}, x_{2}, \ldots, x_{n}\right) .
$$

2. no caso em que a distribuição $F_{\boldsymbol{X}}$ é absolutamente contínua, a função de distribuição condicional é obtida por,

$$
F\left(x_{1}, \ldots, x_{k-1}, x_{k+1}, \ldots, x_{n} \mid x_{k}\right)=\frac{\partial}{\partial x_{k}} F\left(x_{1}, \ldots, x_{k}, \ldots, x_{n}\right)
$$


para $k=1,2, \ldots, n$.

Exemplos bastante conhecidos de distribuições multivariadas são as distribuições chamadas de limitantes inferior de Fréchet, $F_{L}$, e limititante superior de Fréchet, $F_{U}$, definidos respectivamente por,

$$
\begin{aligned}
& F_{L}\left(x_{1}, x_{2}, \ldots, x_{n}\right)=\max \left[\sum_{k=1}^{n} F\left(x_{k}\right)-n+1,0\right]=W\left(F_{1}\left(x_{1}\right), F_{2}\left(x_{2}\right), \ldots, F_{n}\left(x_{n}\right)\right), \\
& F_{U}\left(x_{1}, x_{2}, \ldots, x_{n}\right)=\min \left[F\left(x_{1}\right), F\left(x_{2}\right), \ldots, F\left(x_{n}\right)\right]=M\left(F_{1}\left(x_{1}\right), F_{2}\left(x_{2}\right), \ldots, F_{n}\left(x_{n}\right)\right) .
\end{aligned}
$$

Logo,

$$
W\left(F_{1}\left(x_{1}\right), F_{2}\left(x_{2}\right), \ldots, F_{n}\left(x_{n}\right)\right) \leq F\left(x_{1}, x_{2}, \ldots, x_{n}\right) \leq M\left(F_{1}\left(x_{1}\right), F_{2}\left(x_{2}\right), \ldots, F_{n}\left(x_{n}\right)\right),
$$

em que o limite superior $M$ é sempre uma distribuição conjunta e o inferior $W$ é sempre uma distribuição conjunta para $n=2$. Para $n>2$ são necessárias algumas condições adicionais (Trivedi e Zimmer, 2005). A inequação (1.6) é chamada de desigualdade de Fréchet-Hoeffding.

Outra função que cabe ressaltar é a distribuição $n$-variada produto (independência) dada por,

$$
F\left(x_{1}, x_{2}, \ldots, x_{n}\right)=F_{1}\left(x_{1}\right) F_{2}\left(x_{2}\right) \cdots F_{n}\left(x_{n}\right)
$$

O conceito a seguir será utilizado para mostrar algumas propriedades das cópulas.

Definição 3 (Inversa Generalizada):Seja $F$ uma função de distribuição univariada, defini-se a inversa generalizada ou quasi-inversa de $F$ como uma função 
$F^{-1}: \boldsymbol{I} \rightarrow \mathbb{R}$ tal que,

1. se $t$ está em $R a n F$, então $F^{-1}(t)$ é qualquer número $x \in \mathbb{R}$ tal que $F(x)=t$, isto é, $\forall x \in R a n F$ tem-se que,

$$
F\left(F^{-1}(t)\right)=t
$$

2. se $t \notin R a n F$, então,

$$
F^{-1}(t)=\inf \{x \mid F(x) \geq t\}=\sup \{x \mid F(x) \leq t\}
$$

Essa função será importante na construção de distribuições multivariadas com o uso de funções cópulas como apresentaremos imediatamente a seguir.

\subsection{Funções Cópulas}

A definição mais usual de cópula é uma função de distribuição $C:[0,1]^{n} \rightarrow$ $[0,1]$, isto é, uma função de distribuição multivariada $n$-dimensional definida em um hipercubo unitário de mesma dimensão.

Definição 4 (Função Cópula). Uma função cópula $n$-dimensional é uma função $C:[0,1]^{n} \rightarrow[0,1]$ que satisfaz as seguintes condições,

1. $C$ é não decrescente e contínua a direita em cada componente.

2. Para cada índice $k=1,2, \ldots, n$ tem-se que

$$
\lim _{u_{k} \rightarrow 0^{+}} C\left(u_{1}, u_{2}, \ldots, u_{k-1}, u_{k}, u_{k+1}, \ldots, u_{n}\right)=0
$$


e

$$
\lim _{\forall k, u_{k} \rightarrow 1^{-}} C\left(u_{1}, u_{2}, \ldots, u_{k-1}, u_{k}, u_{k+1}, \ldots, u_{n}\right)=1 .
$$

3. $\operatorname{Vol}_{C\left(S_{1}, S_{2}, \ldots, S_{n}\right)} \geq 0, \forall S_{k}=\left(a_{k}, b_{k}\right] \subseteq \boldsymbol{I}=[0,1], k=1,2, \ldots, n$

4. A $i$-ésima função marginal da cópula é escrita por,

$$
\begin{aligned}
C\left(u_{i}\right) & =\lim _{\forall k \neq i, u_{k} \rightarrow 1^{-}} C\left(u_{1}, \cdots, u_{i-1}, u_{i}, u_{i+1}, \cdots, u_{n}\right) \\
& =C\left(1,1, . .1, u_{i}, 1, \ldots, 1\right) \\
& =u_{i}
\end{aligned}
$$

para $k=1,2, \ldots, n$ e $u_{k} \in[0,1]$

A Condição 4 indica que a $i$-ésima distribuição marginal unidimensional da cópula é uniforme $[0,1]$. Para obtê-la bastar tomar o limite $u_{k} \rightarrow 1^{-}$para variáveis $k=1,2, \ldots i-1, i+1, \ldots n$. A Propriedade 2 estabelece que, se uma das variáveis é igual a zero, então a função $C$ é igual a zero, ou seja, a $C\left(S_{1} \times S_{2} \ldots \times S_{k-1} \times S_{k} \times\right.$ $\left.S_{k+1} \ldots \times S_{n}\right)=0$ se $S_{k}=\{0\}$ para algum $k=1,2, . ., n$. A última condição garante que a probabilidade, induzida por $C$, de qualquer intervalo $n$-dimensional contido no $D o m C$ é não negativo. Todas as propriedades listadas acima são equivalentes a da distribuição multivariada apresentada na seção anterior. Essa característica já era esperada visto que as funções cópulas também são distribuições multivariadas, porém restritas a $[0,1]^{n}$.

Uma relação importante utilizada para demonstrar o Lema 2 do Capítulo 3 é a seguinte inequação de uma cópula $n$-dimensional,

$$
|C(\boldsymbol{u})-C(\boldsymbol{v})| \leq \sum_{k=1}^{n}\left|u_{k}-v_{k}\right|
$$


onde $\boldsymbol{u}, \boldsymbol{v} \in[0,1]^{n}$. Logo, $C$ é uniformemente contínua em $[0,1]^{n}$. Este resultado é proveniente da desigualdade triangular e a demonstração da inequação pode ser vista em Nelsen (2006), p.11.

Um dos pontos que torna uma função cópula interessante é que se $X$ tem distribuição acumulada $F(X \sim F)$ então $F(X)=Y \sim U(0,1)$ (ver Teorema 13 no Apêndice A). Desse modo, um importante teorema que relaciona as distribuições multivariadas com as funções cópulas é o de Sklar.

Teorema 1 (Teorema de Sklar) Seja $F_{\boldsymbol{X}}$ a função de distribuição multivariada com marginais $F_{1}, F_{2}, \ldots, F_{n}$. Então existe uma cópula $C$ tal que para todo $\left(x_{1}, x_{2}, \ldots, x_{n}\right) \in \mathbb{R}^{n}$,

$$
F\left(x_{1}, x_{2}, \ldots, x_{n}\right)=C\left(F_{1}\left(x_{1}\right), F_{2}\left(x_{2}\right), \ldots, F_{n}\left(x_{n}\right)\right) .
$$

Se $F_{1}, F_{2}, \ldots, F_{n}$ são todas absolutamente contínuas, então $C$ é única, caso contrário, $C$ é unicamente determinada em $\operatorname{RanF}_{1} \times \operatorname{RanF}_{2} \times \cdots \times \operatorname{RanF}_{n}$. No sentido contrário, se $C$ é uma função cópula $n$-dimensional e $F_{1}, F_{2}, \ldots, F_{n}$ são funções de distribuição, então a função $F$ definida acima é uma distribuição multivariada $n$-dimensional com marginais $F_{1}, F_{2}, \ldots, F_{n}$.

A prova pode ser encontrada em Sklar (1973).

Por meio do Teorema de Sklar é possível estabelecer uma ligação entre a distribuição multivariada com suas distribuições marginais, a função cópula e suas marginais uniformes. Esta relação é destacada pelo seguinte corolário.

Corolário 1 Seja $F_{\boldsymbol{X}}$ uma função de distribuição multivariada com distribuições marginais contínuas $F_{1}, F_{2}, \ldots, F_{n}$ e $C$ a cópula associada a $F_{\boldsymbol{X}}$, então para qualquer 


$$
\begin{aligned}
& \left(u_{1}, u_{2}, \ldots, u_{n}\right) \in[0,1]^{n} \text { e } x_{1}=F_{1}^{-1}\left(u_{1}\right), x_{2}=F_{2}^{-1}\left(u_{2}\right), \ldots, x_{n}=F_{n}^{-1}\left(u_{n}\right) \text { tem-se que } \\
& \begin{aligned}
F\left(F_{1}^{-1}\left(u_{1}\right), F_{2}^{-1}\left(u_{2}\right), \ldots, F_{n}^{-1}\left(u_{n}\right)\right) & =\operatorname{Pr}\left(F_{1}^{-1}\left(U_{1}\right) \leq x_{1}, F_{2}^{-1}\left(U_{2}\right) \leq x_{2}, \ldots, F_{n}^{-1}\left(U_{n}\right) \leq x_{n}\right) \\
& =\operatorname{Pr}\left(U_{1} \leq u_{1}, U_{2} \leq u_{2}, \ldots, U_{n} \leq u_{n}\right) \\
& =C\left(u_{1}, u_{2}, \ldots, u_{n}\right),
\end{aligned}
\end{aligned}
$$

é a única cópula associada a distribuição $F_{\boldsymbol{X}}$.

Em outras palavras se $\boldsymbol{X} \sim F_{\boldsymbol{X}}$ e $F_{\boldsymbol{X}}$ é contínua então,

$$
\left(F_{1}\left(X_{1}\right), F_{2}\left(X_{2}\right), \ldots, F_{n}\left(X_{n}\right)\right) \sim C
$$

e no sentido contrário se $\boldsymbol{U} \sim C$ então,

$$
\left(F_{1}^{-1}\left(U_{1}\right), F_{2}^{-1}\left(U_{2}\right), \ldots, F_{n}^{-1}\left(U_{n}\right)\right) \sim F_{\boldsymbol{X}}
$$

Do Teorema 1 e do Corolário 1, temos que uma distribuição multivariada $F_{\boldsymbol{X}}$ associada a $\left(X_{1}, X_{2}, \ldots, X_{n}\right)$ com marginais $F_{1}, F_{2}, \ldots, F_{n}$ e cópula associada $C_{\theta}$ que pode ser construída através de (1.9),

$$
F\left(x_{1}, x_{2}, \ldots, x_{n}\right)=C\left(F_{1}\left(x_{1}\right), F_{2}\left(x_{2}\right), \ldots, F_{n}\left(x_{n}\right) ; \theta\right),
$$

em que $\theta$ é o parâmetro ou o conjunto de parâmetros da função cópula. Ele é responsável por configurar a estrutura de dependência entre as marginais. Por consequência disso, $\theta$ é chamado de parâmetro de dependência. Trivedi e Zimmer (2005) destacam que a vantagem do uso de cópulas reside no fato de que as funções marginais $F_{1}\left(x_{1}\right), F_{2}\left(x_{2}\right), \ldots, F_{n}\left(x_{n}\right)$ associadas a cópula $C_{\theta}$ podem pertencer a qualquer família de distribuição, independentemente da função cópula.

Os resultados apresentados acima permitem estabelecer um método de cons- 
truir cópulas a partir de uma distribuição conjunta. Este método pode ser visto por meio do seguinte exemplo.

Exemplo 1 (Nelsen, 2005). Considere a seguinte função bivariada $F_{\boldsymbol{X}}$ descrita por,

$$
F\left(x_{1}, x_{2}\right)= \begin{cases}\frac{\left(x_{1}+1\right)\left(e^{x_{2}}-1\right)}{x_{1}+2 e^{x_{2}}-1}, & \text { se }\left(x_{1}, x_{2}\right) \in[-1,1] \times[0, \infty) \\ 1-e^{-x_{2}}, & \text { se }\left(x_{1}, x_{2}\right) \in[1, \infty) \times[0, \infty) \\ 0, & \text { caso contrário. }\end{cases}
$$

Tomando os limites $\lim _{x_{i \neq j} \rightarrow \infty} F_{j}$ para $i=1,2$ obtém-se as marginais $F_{1}$ e $F_{2}$ dadas por,

$$
\begin{gathered}
F_{1}\left(x_{1}\right)= \begin{cases}0, & \text { se } x_{1}<-1, \\
\left(x_{1}+1\right) / 2, & \text { se } x_{1} \in[-1,1], \\
1, & \text { se } x_{1}>1 .\end{cases} \\
F_{2}\left(x_{2}\right)= \begin{cases}0, & \text { se } x_{2}<0 \\
1-e^{-x_{2},}, & \text { se } x_{2} \geq 0 .\end{cases}
\end{gathered}
$$

As inversas de $F_{1}$ e $F_{2}$ são expressas por,

$$
\begin{aligned}
& F_{1}^{-1}\left(u_{1}\right)=2 u_{1}-1 \\
& F_{2}^{-1}\left(u_{2}\right)=-\ln \left(1-u_{2}\right),
\end{aligned}
$$

em que $\left(u_{1}, u_{2}\right) \in[0,1]^{2}$ e substituindo na função $F_{\boldsymbol{X}}$, obtém-se a seguinte cópula a partir do Corolário 1 ,

$$
C\left(u_{1}, u_{2}\right)=\frac{u_{1} u_{2}}{u_{1}+u_{2}-u_{1} u_{2}} .
$$




\subsection{Algumas Propriedades de Cópulas}

Nesta seção apresentamos algumas propriedades das funções cópulas, dentre elas a simetria, cópula sobrevivência e a cópula condicional. Esta última propriedade será de vital importância para a construção dos resultados apresentados nos Capítulos 2, 3 e 4.

\subsubsection{Cópula Sobrevivência}

Em muitas aplicações, em especial em estudos atuariais e em análise de risco, a variável aleatória de interesse é representada pelo tempo de vida restante ou o risco de algum fenômeno ocorrer acima um determinado limiar. Por exemplo, poderia-se ter o interesse em estimar a probabilidade da perda do valor de um ativo ser maior que um determinado limiar $u$. A esta função denomina-se por função sobrevivência (survival function ou reliability function). No caso univariado, isto é $n=1$, a função sobrevivência, $\bar{F}_{\boldsymbol{X}}$, é expressa da seguinte forma,

$$
\bar{F}_{X}=\operatorname{Pr}(X>x)=1-F(x) .
$$

Para $n=2$,

$$
\bar{F}\left(x_{1}, x_{2}\right)=1-F\left(x_{1}\right)-F\left(x_{2}\right)+F\left(x_{1}, x_{2}\right) .
$$

Já para $n=3$,

$\bar{F}\left(x_{1}, x_{2}, x_{3}\right)=1-F\left(x_{1}\right)-F\left(x_{2}\right)-F\left(x_{3}\right)+F\left(x_{1}, x_{2}\right)+F\left(x_{1}, x_{3}\right)+F\left(x_{2}, x_{3}\right)-F\left(x_{1}, x_{2}, x_{3}\right)$.

As marginais de $\bar{F}_{\boldsymbol{X}}$ são representadas por $\lim _{\forall x_{k \neq j} \rightarrow \infty} \bar{F}_{\boldsymbol{X}}=\bar{F}_{j}$. A partir disso é possível estabelecer uma relação entre a função sobrevivência multivariada e a função cópula. Para isso, considere a cópula $C$ associada ao vetor de variáveis 
aleatórias $\left(X_{1}, X_{2}\right)$, então temos que,

$$
\begin{aligned}
\bar{F}\left(x_{1}, x_{2}\right) & =1-F_{1}\left(x_{1}\right)-F_{2}\left(x_{2}\right)+F\left(x_{1}, x_{2}\right) \\
& =\bar{F}_{1}\left(x_{1}\right)+\bar{F}_{2}\left(x_{2}\right)-1+C\left(F_{1}\left(x_{1}\right), F_{2}\left(x_{2}\right)\right) \\
& =\bar{F}_{1}\left(x_{1}\right)+\bar{F}_{2}\left(x_{2}\right)-1+C\left(1-\bar{F}_{1}\left(x_{1}\right), 1-\bar{F}_{2}\left(x_{2}\right)\right) .
\end{aligned}
$$

Assim sendo, substituindo $u_{1}=\bar{F}\left(x_{1}\right)$ e $u_{2}=\bar{F}\left(x_{2}\right)$ podemos definir a função cópula sobrevivência $C: \boldsymbol{I}^{2} \longrightarrow \boldsymbol{I}$ da seguinte forma,

$$
\bar{C}\left(u_{1}, u_{2}\right)=u_{1}+u_{2}-1+C\left(1-u_{1}, 1-u_{2}\right),
$$

Logo, tem-se que,

$$
\bar{F}\left(x_{1}, x_{2}\right)=\bar{C}\left(\bar{F}\left(x_{1}\right), \bar{F}\left(x_{2}\right)\right),
$$

$\bar{C}\left(u_{1}, u_{2}\right)=\operatorname{Pr}\left(U_{1} \geq u_{1}, U_{2} \geq u_{2}\right)$. Note que a função cópula $\bar{C}$ também é uma cópula visto que atende as condições da Definição 4. O mesmo raciocínio pode ser empregado na construção da cópula sobrevivência para $n \geq 3$.

Uma propriedade interessante das cópulas é a sua invariância em relação as transformações estritamente monotônicas nas suas variáveis. Para ilustrar melhor o caso segue-se o teorema.

Teorema 2 Sejam $X_{1}$ e $X_{2}$ variáveis aleatórias contínuas com a cópula denotada por $C_{X_{1}, X_{2}}$. Se $\alpha$ e $\beta$ são funções estritamente crescente em $\operatorname{Ran} X_{1}$ e em $\operatorname{Ran} X_{2}$ respectivamente, então $C_{\alpha\left(X_{1}\right), \beta\left(X_{2}\right)}=C_{X_{1}, X_{2}}$. Logo, $C_{X_{1}, X_{2}}$ é invariante a transformação estritamente crescente para $X_{1}$ e $X_{2}$.

Prova. Sejam $F_{1}, G_{1}, F_{2}$ e $G_{2}$ as funções de distribuição de $X_{1}, \alpha\left(X_{1}\right), X_{2}$ e $\beta\left(X_{2}\right)$, respectivamente. Como $\alpha$ e $\beta$ são estritamente crescente tem-se que, $F_{2}\left(x_{2}\right)=P\left[\alpha\left(X_{1}\right) \leq x_{1}\right]=P\left[X_{1} \leq \alpha^{-1}\left(x_{1}\right)\right]=F_{1}\left(\alpha^{-1}\left(x_{1}\right)\right)$ e da mesma forma 
tem-se também que $G_{2}\left(x_{2}\right)=G_{1}\left(\beta^{-1}\left(x_{2}\right)\right)$. Logo, para qualquer $x_{1}, x_{2} \in \mathbb{R}$,

$$
\begin{aligned}
C_{\alpha\left(X_{1}\right), \beta\left(X_{2}\right)}\left(F_{2}\left(x_{1}\right), G_{2}\left(x_{2}\right)\right) & =P\left[\alpha\left(X_{1}\right) \leq x_{1}, \beta\left(X_{2}\right) \leq x_{2}\right] \\
& =P\left[X_{1} \leq \alpha^{-1}\left(x_{1}\right), X_{2} \leq \beta^{-1}\left(x_{2}\right)\right] \\
& =C_{X_{1}, X_{2}}\left(F_{1}\left(\alpha^{-1}\left(x_{1}\right)\right), G_{1}\left(\beta^{-1}\left(x_{2}\right)\right)\right) \\
& =C_{X_{1}, X_{2}}\left(F_{2}\left(x_{1}\right), G_{2}\left(x_{2}\right)\right) .
\end{aligned}
$$

Como $X_{1}$ e $X_{2}$ são contínuas e $R a n F_{2}=R a n G_{2}=\boldsymbol{I}$ então segue que $C_{\alpha\left(X_{1}\right), \beta\left(X_{2}\right)}=$ $C_{X_{1}, X_{2}}$ em $\boldsymbol{I}^{2}$.

No caso das distribuições univariadas, dizemos que $X$ é simétrica em relação a $a$ se sua função de distribuição possuir valores equivalentes para $P[X-a \leq x]=$ $\operatorname{Pr}[a-X \leq x]$ e quando $X$ é contínua temos que,

$$
F(a+x)=\bar{F}(a-x)
$$

Entretanto, quando lidamos com o caso bivariado a noção de simetria muda. Nelsen (2006) destaca os seguintes três tipos de simetria.

Definição 5 Sejam $X_{1}$ e $X_{2}$ variáveis aleatórias e $(a, b) \in \mathbb{R}^{2}$,

1. $\left(X_{1}, X_{2}\right)$ é marginalmente simétrico sobre $(a, b)$ se $X_{1}$ e $X_{2}$ são simétricos com relação a $a$ e $b$ respectivamente.

2. $\left(X_{1}, X_{2}\right)$ é radialmente simétrico sobre $(a, b)$ se a distribuição conjunta $F_{\boldsymbol{X}}$ em $X_{1}-a$ e $X_{2}-b$ possui os mesmo valores que em $a-X_{1}$ e $b-X_{2}$.

3. $\left(X_{1}, X_{2}\right)$ é conjuntamente simétrico sobre $(a, b)$ se os seguintes pares de variáveis aleatórias possuem o mesmo valor em $\left(X_{1}-a, X_{2}-b\right),\left(X_{1}-a, b-X_{2}\right)$, $\left(a-X_{1}, X_{2}-b\right)$ e $\left(a-X_{1}, b-X_{2}\right)$. 
Lema 1 Sejam $X_{1}$ e $X_{2}$ variáveis aleatórias contínuas com distribuição conjunta dada por $F_{\boldsymbol{X}}$ e marginais $F_{1}$ e $F_{2}$ respectivamente e seja também $(a, b) \in \mathbb{R}^{2}$ então, $\left(X_{1}, X_{2}\right)$ são radialmente simétrico em relação a $(a, b)$ se e somente se,

$$
F\left(a+x_{1}, b+x_{2}\right)=\bar{F}\left(a-x_{1}, b-x_{2}\right) \forall\left(x_{1}, x_{2}\right) \in \mathbb{R}^{2}
$$

Teorema 3 Sejam $X_{1}$ e $X_{2}$ variáveis aleatórias contínuas com distribuição conjunta $F_{\boldsymbol{X}}$, marginais $F_{1}$ e $F_{2}$ respectivamente e cópula $C$. Além disso, suponha que $X_{1}$ e $X_{2}$ são simétricas em relação $a$ e $b$ respectivamente, então $\left(X_{1}, X_{2}\right)$ é radialmente simétrico em relação $(a, b)$, se e somente se,

$$
C\left(u_{1}, u_{2}\right)=u_{1}+u_{2}-1+C\left(1-u_{1}, 1-u_{2}\right) \forall\left(u_{1}, u_{2}\right) \in \boldsymbol{I}^{2}
$$

isto é, $C\left(u_{1}, u_{2}\right)=\bar{C}\left(u_{1}, u_{2}\right)$.

Prova. A partir da equação (1.14) do Lema 1 temos a seguinte equivalência,

$$
\begin{aligned}
& H\left(a+x_{1}, b+x_{2}\right) \\
& =\bar{H}\left(a-x_{1}, b-x_{2}\right) \forall\left(x_{1}, x_{2}\right) \in \mathbb{R}^{2} \text { (Aplicando a relação } 1.12 \text { no lado direito) } \\
& \Leftrightarrow C\left(F_{1}\left(a+x_{2}\right), F_{2}\left(b+x_{2}\right)\right)=\bar{C}\left(\bar{F}_{1}\left(a-x_{1}\right), \bar{F}_{2}\left(b-x_{2}\right)\right) \text { (Aplicando a relação 1.13) } \\
& \Leftrightarrow C\left(F_{1}\left(a+x_{2}\right), F_{2}\left(b+x_{2}\right)\right)=\bar{C}\left(F_{1}\left(a+x_{1}\right), F_{2}\left(b+x_{2}\right)\right) \text { (Aplicando o Teorema 2) } \\
& \Leftrightarrow C\left(u_{1}, u_{2}\right)=\bar{C}\left(u_{1}, u_{2}\right) \forall\left(u_{1}, u_{2}\right) \in \boldsymbol{I}^{2} .
\end{aligned}
$$




\subsubsection{Cópula Condicional}

De modo geral, toda cópula contínua $C$ pode ser escrita como:

$$
C\left(u_{1}, u_{2}, \ldots, u_{n}\right)=A_{C}\left(u_{1}, u_{2}, \ldots, u_{n}\right)+S_{C}\left(u_{1}, u_{2}, \ldots, u_{n}\right),
$$

em que sua parte absolutamente contínua é dada por,

$$
A_{C}\left(u_{1}, u_{2}, \ldots, u_{n}\right)=\int_{0}^{u_{1}} \int_{0}^{u_{2}} \ldots \int_{0}^{u_{n}} \frac{\partial^{n}}{\partial s_{1} \partial s_{2}, \ldots, \partial s_{n}} C\left(s_{1}, s_{2}, \ldots, s_{n}\right) d s_{1} d s_{2} \ldots d s_{n}
$$

e sua parte singular dada por,

$$
S_{C}\left(u_{1}, u_{2}\right)=C\left(u_{1}, u_{2}\right)-A_{C}\left(u_{1}, u_{2}\right) .
$$

No caso das cópulas, as marginais $u_{1}, u_{2}, \ldots, u_{n}$ são sempre contínuas, o que não ocorre usualmente nas distribuições multivariadas. Se $C \equiv A_{C}$ em todo $\boldsymbol{I}^{n}$, então é dita como absolutamente contínua. Neste caso, a densidade de $C$ é dada por,

$$
c\left(u_{1}, u_{2}, \cdots, u_{n}\right)=\frac{\partial^{n} C\left(u_{1}, u_{2}, \cdots, u_{n}\right)}{\partial u_{1} \partial u_{2} \cdots \partial u_{n}} .
$$

No outro caso, se $C \equiv S_{C}$ em todo $\boldsymbol{I}^{n}$ então $C$ é dita singular e temos que $\partial^{n} C\left(u_{1}, u_{2}, \ldots, u_{n}\right) / \partial u_{1} \partial u_{2}, \ldots, \partial u_{n}=0$ em quase todo o hiperespaço $\boldsymbol{I}^{n}$.

No caso de cópulas absolutamente contínuas, a densidade bivariada $f_{12}$ de $x_{1}$ e $x_{2}$ pode ser expressa em termos da densidade da cópula. Para tal, utilizamos o Teorema 1 e derivamos a cópula bivariada $C$ com relação a $x_{1}$ e $x_{2}$ respectivamente,

$$
\begin{aligned}
f_{12}\left(x_{1}, x_{2}\right) & =\frac{\partial^{2} F_{12}\left(x_{1}, x_{2}\right)}{\partial x_{1} \partial x_{2}}, \\
& =\frac{\partial^{2} C\left(u_{1}, u_{2}\right)}{\partial u_{1} \partial u_{2}} \frac{\partial u_{1}}{\partial x_{1}} \frac{\partial u_{2}}{\partial x_{2}} .
\end{aligned}
$$


A expressão final para a densidade bivariada $f_{12}$ em termos da densidade da cópula fica,

$$
f_{12}\left(x_{1}, x_{2}\right)=c\left(F_{1}\left(x_{1}\right), F_{2}\left(x_{2}\right)\right) f_{1}\left(x_{1}\right) f_{2}\left(x_{2}\right)
$$

Assim, a densidade bivariada pode ser decomposta pela densidade de suas marginais e a função densidade da cópula. Esta função é responsável por introduzir a estrutura entre as variáveis aleatórias $X_{1}$ e $X_{2}$. A formulação (1.18) será utilizada na construção de distribuições multivariadas apresentada no capítulo seguinte.

A equação (1.16) permite o desenvolvimento de um algoritmo mais genérico para geração de vetores aleatórios com uma cópula estabelecida. Por meio deste algoritmo é possível aplicar a técnica de simulação de Monte Carlo, oriunda de distribuições multivariadas.

Para gerar as observações $\left(x_{1}, x_{2}\right)$ de um vetor de variáveis aleatórias $\left(X_{1}, X_{2}\right)$ com distribuição conjunta $F_{\boldsymbol{X}}$ evocaremos o Teorema de Sklar para estabelecer a equivalência expressa na equação (1.10). Utilizando o Corolário 1 podemos trocar o vetor de variáveis aleatórias pelo seguinte vetor $\left(U_{1}, U_{2}\right)$. Porém, agora eles são gerados pela cópula $C$. O procedimento para amostrar um par de observações $\left(u_{1}, u_{2}\right)$ com distribuição uniforme $(0,1)$, contudo não independentes, é através do uso da função de distribuição condicional $P\left[U_{2} \leq u_{2} \mid U_{1}=u_{1}\right]$. Esta função pode ser obtida mediante a seguinte operação,

$$
\begin{aligned}
D_{1} C\left(u_{1}, u_{2}\right) & :=\frac{\partial C\left(u_{1}, u_{2}\right)}{\partial u_{1}} \\
& =\lim _{\Delta \rightarrow 0} \frac{C\left(u_{1}+\Delta, u_{2}\right)-C\left(u_{1}, u_{2}\right)}{\Delta} \\
& =\lim _{\Delta \rightarrow 0} \frac{P\left[U_{1} \leq u_{1}+\Delta, U_{2} \leq u_{2}\right]-P\left[U_{1} \leq u_{1}, U_{2} \leq u_{2}\right]}{\Delta} \\
& =\lim _{\Delta \rightarrow 0} \frac{P\left[u_{1} \leq U_{1} \leq u_{1}+\Delta, U_{2} \leq u_{2}\right]}{P\left[u_{1} \leq U_{1} \leq u_{1}+\Delta\right]} \\
& =P\left[U_{2} \leq u_{2} \mid U_{1}=u_{1}\right] \\
& =C_{2 \mid 1}\left(u_{2} \mid u_{1}\right)
\end{aligned}
$$


O algoritmo para gerar $M$ amostras $\left(U_{1}, U_{2}\right) \sim C$ segue a partir da função de distribuição acumulada condicional (1.19) aplicando os seguintes passos:

\section{Algoritmo 1 (Simulação: Cópula bidimensional)}

Para cada $i=1,2, \ldots, M$ execute

1 Simule duas observações independentes com distribuição uniforme $u_{1} \sim U(0,1)$ e $t \sim U(0,1)$.

2 Compute $u_{2}=C^{-1}\left(u_{2} \mid u_{1}\right)(t)=D_{1} C^{-1}\left(u_{1}, u_{2}\right)(t)$.

3 Registre o i-ésimo par amostrado por $\left(u_{1}, u_{2}\right)_{(i)}$.

Este algoritmo ainda pode ser generalizado para cópulas n-dimensional utilizandose do mesmo princípio. O processo é feito de forma recursiva. Para isso, considere o caso geral com uma cópula $n$-dimensional, absolutamente continua. Com a notação,

$$
C_{k}\left(u_{1}, u_{2}, \ldots, u_{k}\right)=C\left(u_{1}, u_{2}, \ldots, u_{k}, 1, \ldots, 1\right), \operatorname{com} k=2, \ldots, n-1
$$

para, uma marginal $k$-dimensional de $C$, temos que quando $k=3$,

$$
\begin{aligned}
D_{1} C\left(u_{1}, u_{2}, u_{3}\right) & :=\lim _{\Delta \rightarrow 0} \frac{C\left(u_{1}+\Delta, u_{2}, u_{3}\right)-C\left(u_{1}, u_{2}, u_{3}\right)}{\Delta}, \\
& =\lim _{\Delta \rightarrow 0} \frac{P\left(U_{1} \leq u_{1}+\Delta, U_{2} \leq u_{2}, U_{3} \leq u_{3}\right)-P\left(U_{1} \leq u_{1}, U_{2} \leq u_{2}, U_{3} \leq u_{3}\right)}{\Delta}, \\
& =\lim _{\Delta \rightarrow 0} \frac{P\left(u_{1} \leq U_{1} \leq u_{1}+\Delta, U_{2} \leq u_{2}, U_{3} \leq u_{3}\right)}{P\left(u_{1} \leq U_{1} \leq u_{1}+\Delta\right)} \\
& =P\left(U_{2} \leq u_{2}, U_{3} \leq u_{3} \mid U_{1}=u_{1}\right), \\
& =C_{23 \mid 1}\left(u_{2}, u_{3} \mid u_{1}\right) .
\end{aligned}
$$


Se derivarmos a equação (1.20) novamente, agora com relação a $u_{2}$,

$$
\begin{aligned}
D_{1,2} C_{3}\left(u_{1}, u_{2}, u_{3}\right) & :=\frac{\partial^{2}}{\partial u_{1} \partial u_{2}} C_{3}\left(u_{1}, u_{2}, u_{3}\right), \\
& =\frac{\partial}{\partial u_{2}} C_{23 \mid 1}\left(u_{2}, u_{3} \mid u_{1}\right), \quad \text { Pela eq.1.20), } \\
& =\lim _{\Delta \rightarrow 0} \frac{P\left(u_{2} \leq U_{2} \leq U_{2}+\Delta, U_{3} \leq u_{3} \mid U_{1}=u_{1}\right)}{P\left(u_{2} \leq U_{2} \leq u_{2}+\Delta\right)} \\
& =\lim _{\Delta \rightarrow 0} P\left(U_{3} \leq u_{3} \mid U_{1}=u_{1}, u_{2} \leq U_{2} \leq u_{2}+\Delta\right) \\
& \times \frac{P\left(u_{2} \leq U_{2} \leq u_{2}+\Delta \mid U_{1}=u_{1}\right)}{P\left(u_{2} \leq U_{2} \leq u_{2}+\Delta\right)} \\
& =C_{3 \mid 12}\left(u_{3} \mid u_{1}, u_{2}\right) D_{1,2} C\left(u_{1}, u_{2}\right)
\end{aligned}
$$

Logo, a distribuição condicional de $U_{3}$ dado $U_{1}$ e $U_{2}$ pode ser expressa por,

$$
C_{3 \mid 12}\left(u_{3} \mid u_{1}, u_{2}\right)=\frac{D_{1,2} C_{3}\left(u_{1}, u_{2}, u_{3}\right)}{D_{1,2} C_{2}\left(u_{1}, u_{2}\right)}
$$

A mesma construção ocorre para a distribuição condicional de $U_{4}$ dado $U_{1}, U_{2}$ e $U_{3}$. Estendendo para o caso geral temos que para vetor aleatório $\left(U_{1}, U_{2}, \ldots, U_{n}\right)$ com distribuição $C$, a distribuição condicional de $U_{k}$ dado $U_{1}, U_{2}, \ldots, U_{k-1}$ possui a seguinte expressão,

$$
\begin{aligned}
C_{k}\left(u_{k} \mid u_{1}, u_{2}, \cdots, u_{k-1}\right) & =D_{1:(k-1)} C_{k}\left(u_{1}, u_{2}, \cdots, u_{k}\right) / D_{1:(k-1)} C_{k-1}\left(u_{1}, u_{2}, \cdots, u_{k-1}\right) \\
& =P\left(U_{k} \leq u_{k} \mid U_{1}=u_{1}, \cdots, U_{k-1}=u_{k-1}\right) .
\end{aligned}
$$

Sendo o denominador diferente de zero e existindo tanto o numerador quanto o denominador, os seguintes passos do algoritmo para gerar $M$ amostras de $C$ são descritos abaixo,

\section{Algoritmo 2 (Simulação: Cópula $n$-dimensional)}

Para cada $i=1,2, \ldots, M$ execute 
1 Simule uma observação com $u_{1} \sim U(0,1)$.

2 Simule uma observação com $u_{2} \sim C_{2}\left(\cdot \mid u_{1}\right)$.

$\vdots$

n Simule uma observação com $u_{n} \sim C_{n}\left(\cdot \mid u_{1}, u_{2}, \ldots, u_{n-1}\right)$. $\mathbf{n}+\mathbf{1}$ Registre a i-ésima amostra $\left(u_{1}, u_{2}, \ldots, u_{n}\right)_{(i)}$

A simulação de vetores aleatórios associados a cópulas é de grande utilidade nas aplicações em diversas áreas. As amostras geradas pela simulação permite estudar uma série de aspectos como o comportamento de sistemas, na comparação de métodos, em testes de robusteza, em resultados de propriedades assintóticas de pequenas amostras (Nelsen, 2006).

Pelo Teorema 1, a construção de distribuições multivariadas pode ser segregada entre a estrutura de dependência e a definição das distribuições marginais. As funções cópulas são responsáveis por configurar a estrutura de dependência. Em razão disso existem diversas formas de mensurar a dependência entre as componentes de um vetor aleatório. Na próxima seção abordamos algumas delas.

\subsection{Medidas de Dependência}

Tendo em vista a unicidade da equação (1.10), quando $F_{1}\left(x_{1}\right), F_{2}\left(x_{2}\right), \ldots, F_{n}\left(x_{n}\right)$ são contínuas, é possível interpretar uma função cópula como uma função de dependência entre variáveis aleatórias. Desta forma, a inserção de cópula na modelagem da dependência tem uma forte relação com estruturas de associações. Majoritariamente as famílias de cópulas dependem de um ou mais parâmetros $\theta$, os quais tem relação com algumas medidas de correlação entre as variáveis aleatórias, os 
quais abordaremos a diante, bem como algumas de suas propriedades. Nesta seção nos limitaremos aos casos bivariados, embora possam ser estendidos para os casos multivariados.

Tomando-se o vetor de variáveis aleatórias $\left(X_{1}, X_{2}\right)$, é dito que elas são dependentes ou associadas se não são independentes no sentido que $F\left(x_{1}, x_{2}\right) \neq$ $F_{1}\left(x_{1}\right) F_{2}\left(x_{2}\right)$. Sendo $\delta\left(X_{1}, X_{2}\right)$ uma medida de dependência, Embrechts et al. (2001b) destacam-se quatro propriedades desejáveis para $\delta$ :

1. Simetria: $\delta\left(X_{1}, X_{2}\right)=\delta\left(X_{2}, X_{1}\right)$.

2. Normalização: $-1 \leq \delta\left(X_{1}, X_{2}\right) \leq 1$.

3. Comonotonicidade: Se $\delta\left(X_{1}, X_{2}\right)=1 \Leftrightarrow X_{1}, X_{2}$ são comonotônicas.

Contramonoticidade: Se $\delta\left(X_{1}, X_{2}\right)=-1 \Leftrightarrow X_{1}, X_{2}$ são contramonotônicas.

4. Para $T: \mathbb{R} \rightarrow \mathbb{R}$ estritamente monótono no domínio da variável $X_{1}$ :

$$
\delta\left(T\left(X_{1}\right), X_{2}\right)= \begin{cases}\delta\left(X_{1}, X_{2}\right) & \text { se } T \text { é crescente } \\ -\delta\left(X_{1}, X_{2}\right) & \text { se } T \text { é decrescente }\end{cases}
$$

As medidas mais usuais quanto abordagem de cópulas são três. A primeira delas é a correlação de Pearson que é definida a seguir.

Definição 6 (Coeficiente de Correlação Linear de Pearson). Seja $X_{1}, X_{2}$ um par de variáveis aleatórias, ambos com variância finita, a correlação de Pearson é expressa por,

$$
\rho\left(X_{1}, X_{2}\right)=\frac{\operatorname{Cov}\left[X_{1}, X_{2}\right]}{\sqrt{\operatorname{Var}\left[X_{1}\right] \operatorname{Var}\left[X_{2}\right]}},
$$

em que $\operatorname{Cov}\left[X_{1}, X_{2}\right]=\mathbb{E}\left[X_{1} X_{2}\right]-\mathbb{E}\left[X_{1}\right] \mathbb{E}\left[X_{2}\right]$ é a covariância e $\operatorname{Var}\left[X_{1}\right], \mathbb{V}$ ar $\left[X_{2}\right]>$ 0 são as variâncias de $X_{1}$ e $X_{2}$ respectivamente. 
O conceito acima pode ser estendido para o caso multivariado, neste caso a correlação será dada através de uma matriz simétrica e positiva definida. A correlação de Pearson capta uma estrutura de correlação linear, daí o seu outro nome: coeficiente de correlação linear. Neste caso, se $X_{1}=a X_{2}+b$ em que $a \in \mathbb{R} \backslash\{0\}$ e $b \in \mathbb{R}$ então temos que $\left|\rho\left(X_{1}, X_{2}\right)\right|=1$. A prova deste resultado é imediata, bastando para isso substituir $X_{1}=a X_{2}+b$ na definição. O coeficiente de correlação linear de Pearson atende apenas as propriedades 1 e 2 supracitadas. Devido a sua estrutura linear, $\rho$ possuí uma importante propriedade,

$$
\rho\left(a X_{1}+b, c X_{2}+d\right)=\operatorname{sinal}(a c) \rho\left(X_{1}, X_{2}\right)
$$

onde $a, c \in \mathbb{R} /\{0\}, b, d \in \mathbb{R}$. Esta propriedade mostra que o coeficiente de correlação $\rho$ é invariante apenas por transformações lineares.

Apesar da popularidade de coeficiente de correlação $\rho$, ele padece de severas limitações que são destacadas por alguns autores como Trivedi e Zimmer (2005) e Cherubini et al. (2004). No caso de uma distribuição normal bivariada, a correlação é totalmente informativa. Sendo $\rho=0(\mathbb{C o v}=0)$ há a independência das variáveis. Entretanto, não se pode estender este caso para todas as outras distribuições. A independência apenas implica $\rho=0$, porém, o contrário não é necessariamente válido. Uma segunda limitação é que a variância deve ser finita, no entanto, como destaca Cont (2001), os fatos estilizados em finanças sugerem que muitas distribuições provenientes de séries temporais financeiras não possuem segundo momento finito. Um terceiro problema ocorre devido a não invariância com relação as transformações não lineares. Em virtude de algumas dessas limitações outras medidas de dependência baseadas no conceito de concordância são consideradas.

Sejam $\left(x_{1}, x_{2}\right)$ e $\left(x_{1}^{\prime}, x_{2}^{\prime}\right)$ os pares de duas observações de um vetor, então há a concordância se $x_{1}<x_{1}^{\prime}$ e $x_{2}<x_{2}^{\prime}$ ou o contrário $x_{1}>x_{1}^{\prime}$ e $x_{2}>x_{2}^{\prime}$ ocorre, 
isto é, se um aumento de uma das variáveis promove o aumento da outra variável. No mesmo sentido dizemos que os pares são discordantes se observar $x_{1}<x_{1}^{\prime} \mathrm{e}$ $x_{2}>x_{2}^{\prime}$ ou $x_{1}>x_{1}^{\prime}$ e $x_{2}<x_{2}^{\prime}$. Outra formulação possível para a concordância é $\left(x_{1}-x_{1}^{\prime}\right)\left(x_{2}-x_{2}^{\prime}\right)>0$ para os pares concordantes e $\left(x_{1}-x_{1}^{\prime}\right)\left(x_{2}-x_{2}^{\prime}\right)<0$ para os discordantes.

Uma função importante que surge a partir da ideia de concordância é a chamada função $Q$. Ela mede a diferença da probabilidade de pares concordantes e discordantes entre dois vetores de variáveis aleatórias contínuas com distribuições conjuntas distintas, porém com mesmas marginais dependendo apenas das suas funções cópulas. Esta relação pode ser vista através do seguinte teorema.

Teorema 4 Sejam $\left(X_{1}, Y_{1}\right)$ e $\left(X_{2}, Y_{2}\right)$ dois vetores independentes de variáveis aleatórias contínuas com distribuição conjunta dada por $H_{1}$ e $H_{2}$, respectivamente, com marginais $F_{X}$ para $X_{1}$ e $X_{2}$ e $F_{Y}$ para $Y_{1}$ e $Y_{2}$. Sejam também, $C_{1}$ e $C_{2}$ as cópulas correspondentes aos pares $\left(X_{1}, Y_{1}\right)$ e $\left(X_{2}, Y_{2}\right)$, respectivamente, de forma que $H_{1}=C_{1}\left(F_{X}\left(x_{1}\right), F_{Y}\left(y_{1}\right)\right)$ e $H_{2}=C_{2}\left(F_{X}\left(x_{2}\right), F_{Y}\left(y_{2}\right)\right)$. Seja $Q$ a função denotada pela diferença entre a probabilidade da concordância e discordância dos pares $\left(X_{1}, Y_{1}\right)$ e $\left(X_{2}, Y_{2}\right)$, isto é,

$$
Q=P\left[\left(X_{1}-X_{2}\right)\left(Y_{1}-Y_{2}\right)>0\right]-P\left[\left(X_{1}-X_{2}\right)\left(Y_{1}-Y_{2}\right)<0\right],
$$

então,

$$
Q=Q\left(C_{1}, C_{2}\right)=4 \iint_{I^{2}} C_{2}\left(u_{1}, u_{2}\right) d C_{1}\left(u_{1}, u_{2}\right)-1
$$

Prova. Como as variáveis aleatórias são contínuas

$$
P\left[\left(X_{1}-X_{2}\right)\left(Y_{1}-Y_{2}\right)<0\right]=1-P\left[\left(X_{1}-X_{2}\right)\left(Y_{1}-Y_{2}\right)>0\right]
$$


$\log Q Q$ pode ser escrita por,

$$
Q=2 P\left[\left(X_{1}-X_{2}\right)\left(Y_{1}-Y_{2}\right)>0\right]-1
$$

Tomando $P\left[\left(X_{1}-X_{2}\right)\left(Y_{1}-Y_{2}\right)>0\right]=P\left[X_{1}>X_{2}, Y_{1}>Y_{2}\right]+P\left[X_{1}<X_{2}, Y_{1}<Y_{2}\right]$ e reescrevendo essas probabilidades pela integral avaliada sob a distribuição de um dos pares, tem-se para a primeira parcela a seguinte expressão,

$$
\begin{aligned}
P\left[X_{2}<X_{1}, Y_{2}<Y_{1}\right] & =\iint_{\mathbb{R}^{2}} P\left[X_{2} \leq x, Y_{2} \leq y\right] d C_{1}\left(F_{X}(x), F_{Y}(y)\right) \\
& =\iint_{\mathbb{R}^{2}} C_{2}\left(F_{X}(x), F_{Y}(y)\right) d C_{1}\left(F_{X}(x), F_{Y}(y)\right) .
\end{aligned}
$$

Substituindo $u_{1}=F_{X}(x)$ e $u_{2}=F_{Y}(y)$ obtém-se,

$$
P\left[X_{1}>X_{2}, Y_{1}>Y_{2}\right]=\iint_{I^{2}} C_{2}\left(u_{1}, u_{2}\right) d C_{1}\left(u_{1}, u_{2}\right)
$$

Analogamente para a segunda parcela,

$$
\begin{aligned}
P\left[X_{1}<X_{2}, Y_{1}<Y_{2}\right] & =\iint_{\mathbb{R}^{2}} P\left[X_{2} \geq x, Y_{2} \geq x\right] d C_{1}\left(F_{X}(x), F_{Y}(y)\right) \\
& =\iint_{\mathbb{R}^{2}} \bar{C}_{2}\left(F_{X}(x), F_{Y}(y)\right) d C_{1}\left(F_{X}(x), F_{Y}(y)\right)
\end{aligned}
$$

e utilizando a mesma substituição $u_{1}=F_{X}(x)$ e $u_{2}=F_{Y}(y)$,

$$
\begin{aligned}
P\left[X_{1}<X_{2}, Y_{1}<Y_{2}\right] & =\iint_{I^{2}}\left[1-u_{1}-u_{2}+C_{2}\left(u_{1}, u_{2}\right)\right] d C_{1}\left(u_{1}, u_{2}\right), \\
& =1-\frac{1}{2}-\frac{1}{2}+\iint_{I^{2}} C_{2}\left(u_{1}, u_{2}\right) d C_{1}\left(u_{1}, u_{2}\right), \\
& =\iint_{I^{2}} C_{2}\left(u_{1}, u_{2}\right) d C_{1}\left(u_{1}, u_{2}\right) .
\end{aligned}
$$


Desta forma, temos que,

$$
P\left[\left(X_{1}-X_{2}\right)\left(Y_{1}-Y_{2}\right)>0\right]=2 \iint_{I^{2}} C_{2}\left(u_{1}, u_{2}\right) d C_{1}\left(u_{1}, u_{2}\right)
$$

e portanto,

$$
Q=Q\left(C_{1}, C_{2}\right)=4 \iint_{I^{2}} C_{2}\left(u_{1}, u_{2}\right) d C_{1}\left(u_{1}, u_{2}\right)-1
$$

A função $Q$ é importante para escrever outras medidas de correlação definidas com base a medidas de concordância, elas são: $\tau$ de Kendall e $\rho$ de Spearman.

Definição 7 (Correlação $\tau$ de Kendall). Sendo $X_{1}$ e $X_{2}$ variáveis aleatórias contínuas com cópula $C$, então a medida de correlação de $\tau$ de Kendall é dada por,

$$
\tau\left(X_{1}, X_{2}\right)=\tau(C)=Q(C, C)=4 \iint_{I^{2}} C\left(u_{1}, u_{2}\right) d C\left(u_{1}, u_{2}\right)-1
$$

Observe que a integral da definição acima pode ser vista com o valor esperado de $C\left(U_{1}, U_{2}\right)$ de uma distribuição bivariada com marginais uniformes. Isto é,

$$
\tau_{C}=4 \mathbb{E}\left[C\left(U_{1}, U_{2}\right)\right]-1
$$

Definição 8 (Correlação $\rho_{S}$ de Spearman). Sendo $X_{1}$ e $X_{2}$ variáveis aleatórias contínuas com cópula $C$ e $\Pi\left(u_{1}, u_{2}\right)=u_{1} u_{2}$, então a versão da correlação $\rho_{S}$ de Spearman para o vetor de variáveis aleatórias $\left(X_{1}, X_{2}\right)$ é dada por,

$$
\begin{aligned}
\rho_{S}\left(X_{1}, X_{2}\right)=\rho_{S}(C) & =3 Q(C, \Pi) \\
& =12 \iint_{\mathbf{I}^{2}} u_{1} u_{2} d C\left(u_{1}, u_{2}\right)-3 \\
& =12 \iint_{\mathbf{I}^{2}} C\left(u_{1}, u_{2}\right) d u_{1} d u_{2}-3 .
\end{aligned}
$$


Sendo que a última passagem da equação acima se deve a Propriedade 1 da função $Q$ de intercambiabilidade apresentada anteriormente.

A partir destas duas medidas de correlação baseadas em pares concordantes, Durbin e Stuart (1951) descrevem uma relação entre $\tau(C)$ e $\rho_{S}(C)$ através das seguintes inequações,

$$
\begin{gathered}
\frac{3}{2} \tau(C)-\frac{1}{2} \leq \rho_{S}(C) \leq \frac{1}{2}+\tau_{C}-\frac{1}{2} \tau^{2}(C) \text { para } \tau(C) \geq 0, \\
\frac{1}{2} \tau(C)^{2}+\tau(C)-\frac{1}{2} \leq \rho_{S}(C) \leq \frac{3}{2} \tau(C)+\frac{1}{2} \text { para } \tau(C)<0 .
\end{gathered}
$$

Note que (1.24) e (1.25) somente dependem da cópula $C$, no entanto, a correlação de Pearson depende de $C$ e das distribuições marginais $F_{1}$ e $F_{2}$ de $X_{1}$ e $X_{2}$, respectivamente, pois,

$$
\operatorname{Cov}\left(X_{1}, X_{2}\right)=\iint_{I^{2}} F_{1}^{-1}\left(u_{1}\right) F_{2}^{-2}\left(u_{2}\right) d C\left(u_{1}, u_{2}\right)-\iint_{I^{2}} F_{2}^{-2}\left(u_{2}\right) d u_{1} d u_{2} .
$$

Daí pelo Teorema $2 \tau\left(X_{1}, X_{2}\right)$ e $\rho_{S}\left(X_{1}, X_{2}\right)$ são invariantes por transformações estritamente crescentes.

O conceito de dependência nas caudas superior e inferior de uma distribuição bivariada é muito importante para o estudo da dependência entre valores extremos. Por exemplo, em finanças, um agente pode estar interessado na probabilidade da queda de dois ativos que compõe uma carteira de investimento, dado um certo nível de perda. Este comportamento diz respeito, portanto, a eventos que ocorrem nas caudas da distribuição, ou seja, descreve quão grande o valor de uma variável aleatória aparece concomitantemente com o surgimento de mesma magnitude da outra.

Definição 9 (Coeficiente de Dependência Caudal). Sejam $X_{1}$ e $X_{2}$ variáveis aleatórias contínuas com funções de distribuições $F_{1}$ e $F_{2}$, respectivamente. O 
coeficiente de dependência caudal superior é o limite (se ele existir) da seguinte probabilidade condicional de $X_{2}$,

$$
\lambda_{U}=\lim _{t \rightarrow 1^{-}} P\left[X_{2}>F_{2}^{-1}(t) \mid X_{1}>F_{1}^{-1}(t)\right] .
$$

De forma similar, temos o mesmo caso para o coeficiente de dependência caudal inferior,

$$
\lambda_{L}=\lim _{t \rightarrow 0^{+}} P\left[X_{2} \leq F_{2}^{-1}(t) \mid X_{1} \leq F_{1}^{-1}(t)\right] .
$$

A partir desta definição podemos construir uma relação dessa medida com as cópulas por meio do seguinte teorema.

Teorema 5 Sejam o $X_{1}$ e $X_{2}$ variáveis aleatórias contínuas com distribuição $F_{X_{1}}$ e $F_{X_{2}}$ e $C$ a cópula do par das variáveis $X_{1}$ e $X_{2}$. Se o limite existir então,

$$
\lambda_{U}=2-\lim _{t \rightarrow 1^{-}} \frac{1-C(t, t)}{1-t}
$$

$\mathrm{e}$

$$
\lambda_{L}=\lim _{t \rightarrow 0^{+}} \frac{C(t, t)}{t}
$$


Prova. Pela definição de $\lambda_{U}$, temos que,

$$
\begin{aligned}
\lambda_{U} & =\lim _{t \rightarrow 1^{-}} P\left[X_{2}>F_{2}^{-1}(t) \mid X_{1}>F_{1}^{-1}(t)\right], \\
& =\lim _{t \rightarrow 1^{-}} P\left[F_{2}\left(X_{2}\right)>t \mid F_{1}\left(X_{1}\right)>t\right] \\
& =\lim _{t \rightarrow 1^{-}} \frac{P\left[F_{2}\left(X_{2}\right)>t, F_{1}\left(X_{1}\right)>t\right]}{P\left[F_{1}\left(X_{1}\right)>t\right]} \\
& =\lim _{t \rightarrow 1^{-}} \frac{\bar{C}(t, t)}{1-t} \\
& =\lim _{t \rightarrow 1^{-}} \frac{1-2 t+C(t, t)}{1-t} \\
& =2-\lim _{t \rightarrow 1^{-}} \frac{1-C(t, t)}{1-t}
\end{aligned}
$$

A prova para o caso $\lambda_{L}$ é similar ao anterior. Se $\lambda_{U} \in(0,1]$ é dito que a cópula $C$ possui dependência caudal superior e se $\lambda_{U}=0$, então $C$ não tem dependência caudal superior. O mesmo é válido para o coeficiente caudal $\lambda_{L}$. Em algumas situações em que não há forma fechada para a função cópula é interessante representar a dependência caudal de outro modo. Sendo $C$ a função cópula do vetor $\left(U_{1}, U_{2}\right)$, podemos reescrever $\lambda_{U}$ da seguinte maneira,

$$
\begin{aligned}
\lambda_{U} & =\lim _{u \rightarrow 1^{-1}} \frac{\bar{C}(u, u)}{(1-u)} \\
& =-\lim _{u \rightarrow 1^{-}} \frac{d \bar{C}(u, u)}{d u}, \\
& =-\lim _{u \rightarrow 1^{-}}\left(-2+\left.\frac{\partial}{\partial s} C(s, t)\right|_{s=t=u}+\left.\frac{\partial}{\partial t} C(s, t)\right|_{s=t=u}\right), \\
& =\lim _{u \rightarrow 1^{-}} \operatorname{Pr}\left[U_{2}>u \mid U_{1}=u\right]+\operatorname{Pr}\left[U_{1}>u \mid U_{2}=u\right] .
\end{aligned}
$$

Quando a cópula $C$ tem simetria radial, conforme definido na subseção 1.3.1, isto é $C\left(u_{1}, u_{2}\right)=C\left(u_{2}, u_{1}\right)$, é possível simplificar ainda mais a expressão de $\lambda_{U}$ por,

$$
\lambda_{U}=2 \lim _{u \rightarrow 1^{-}} P\left[U_{2}>u \mid U_{1}=u\right]
$$


de forma similar, obtemos para $\lambda_{L}$,

$$
\lambda_{L}=2 \lim _{u \rightarrow 0^{+}} P\left[U_{2}<u \mid U_{1}=u\right]
$$

De posse destas medidas é possível descrever melhor as características das famílias de cópulas. Na próxima seção segue a apresentação de algumas famílias de cópulas.

\subsection{Principais Famílias de Cópulas Bivariadas}

De acordo com o Teorema de Sklar, uma distribuição multivariada pode ser tratada pela sua estrutura de dependência, cópula, e por suas marginais. O tratamento distinto entre a estrutura de dependência das variáveis e as suas distribuições marginais explica a grande flexibilidade da modelagem via funções cópulas. Isto ocorre tanto nas aplicações empíricas quanto do ponto de vista teórico (Cherubini et al., 2004). Em função disso, há uma enorme quatidade de funções cópulas propostas pela literatura, cada uma com estrutura de dependência particular. Nesta seção, abordaremos as principais delas e suas características fundamentais.

\subsubsection{Cópulas Elementares}

A função cópula mais simples a ser definida é a cópula produto ou da independência П. Sua forma foi utilizada na Definição 8 e é definida por,

$$
\Pi\left(u_{1}, u_{2}\right)=u_{1} u_{2}
$$

em que novamente temos $\left(u_{1}, u_{2}\right) \in[0,1]^{2}=\boldsymbol{I}^{2}$. Ela é utilizada por exemplo na medida de dependência $\rho_{S}$ de Spearman como destacado em (1.25). 


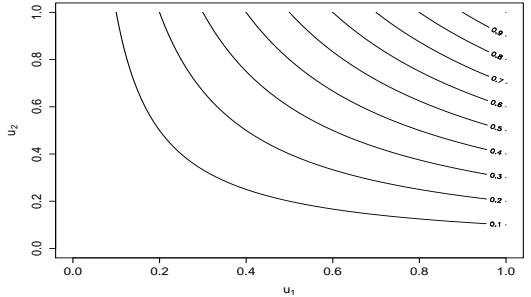

(a) Curvas de Nível

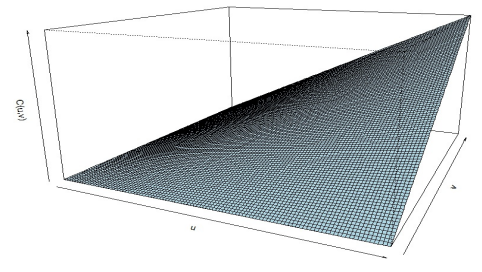

(b) Gráfico 3D

Figura 1.1: Gráfico da cópula П

A Figura 1.1 mostra as curvas de níveis da função cópula (1.31) e seu respectivo gráfico em perspectiva.

A partir da desigualdade de Fréchet-Hoeffding, expressa na inequação (1.6), surgem duas outras cópulas importantes. Realizando as substituições $u_{1}=F\left(x_{1}\right)$ e $u_{2}=F\left(x_{2}\right)$ nas equações (1.5), temos as seguintes cópulas com seus respectivos gráficos,

$$
C_{U}\left(u_{1}, u_{2}\right)=\min \left\{u_{1}, u_{2}\right\}
$$

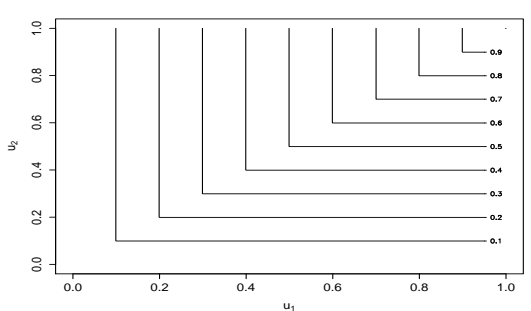

(a) Curvas de Nível

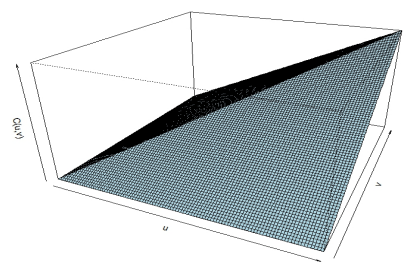

(b) Gráfico 3D

Figura 1.2: Gráfico da cópula $C_{U}$

$$
C_{L}\left(u_{1}, u_{2}\right)=\max \left\{u_{1}+u_{2}-1,0\right\}
$$




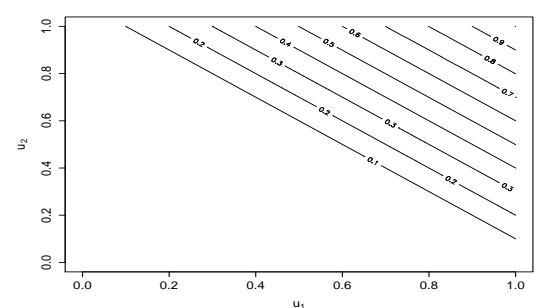

(a) Curvas de Nível

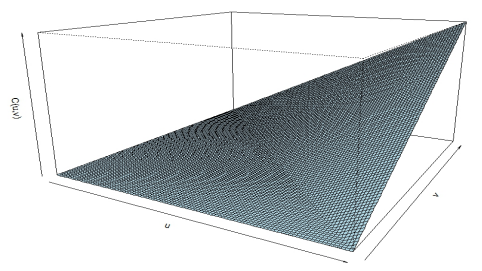

(b) Gráfico 3D

Figura 1.3: Gráfico da cópula $C_{L}$

Por consequência da inequação (1.6), a seguinte desigualdade também ocorre para uma função cópula $C$ qualquer,

$$
C_{L}\left(u_{1}, u_{2}\right) \leq C\left(u_{1}, u_{2}\right) \leq C_{U}\left(u_{1}, u_{2}\right)
$$

$\mathrm{e}$

$$
C_{L}\left(u_{1}, u_{2}\right) \leq \Pi\left(u_{1}, u_{2}\right) \leq C_{U}\left(u_{1}, u_{2}\right)
$$

Sendo o vetor aleatório $\left(X_{1}, X_{2}\right)$ associado à cópula $C$ a seguinte relação é observada,

$$
\begin{aligned}
& C=C_{L} \quad \Rightarrow \quad \tau\left(X_{1}, X_{2}\right)=\rho\left(X_{1}, X_{2}\right)=-1 \\
& C=\Pi \quad \Rightarrow \quad \tau\left(X_{1}, X_{2}\right)=\rho\left(X_{1}, X_{2}\right)=0 \\
& C=C_{U} \quad \Rightarrow \quad \tau\left(X_{1}, X_{2}\right)=\rho\left(X_{1}, X_{2}\right)=1 .
\end{aligned}
$$

Se $X_{1}$ e $X_{2}$ forem variáveis aleatórias contínuas, então a relação inversa também é verificada. A prova dessa relação pode ser vista em Embrechts et al. (2001b).

\subsubsection{Cópulas Elípticas}

A classe de distribuições elípticas oferece uma variedade de distribuições multivariadas e que compartilham de muitas propriedades com a distribuição normal 
multivariada, o que lhe concebe algumas vantagens como a facilidade de simular dados provenientes destas distribuições (Embrechts et al., 2001b). As cópulas elípticas são oriundas de distribuições elípticas multivariadas. A definição de uma distribuição elíptica é descrita abaixo.

Definição 10 (Classe Distribuições Elípticas.) Seja $\boldsymbol{X}$ um vetor de variáveis aleatórias $n$-dimensional, $\mu \in \mathbb{R}$ e $\Sigma$ uma matriz simétrica $n \times n$ não negativa definida. Então, se a função característica $\varphi_{\boldsymbol{X}-\mu}(\boldsymbol{t})$ da variável $\boldsymbol{X}-\mu$ é função de uma estrutura quadrática $\boldsymbol{t}^{\prime} \Sigma \boldsymbol{t}$, isto é, $\varphi_{\boldsymbol{X}-\mu}(\boldsymbol{t})=\phi\left(\boldsymbol{t}^{\prime} \Sigma \boldsymbol{t}\right)$, então $\boldsymbol{X}$ pertence à classe de distribuições elípticas com parâmetros $\mu, \Sigma$, e $\phi$ e é denotado por $\boldsymbol{X} \sim E_{n}(\mu, \Sigma, \phi)$.

Desta forma, os gráficos de contorno destas distribuições têm o formato de uma elipsóide no $\mathbb{R}^{n}$, como destaca Jondeau et al. (2007). A classe das cópulas elípticas inclui algumas distribuições elípticas multivariadas bem conhecidas como a distribuição Gaussiana e a t-Student, por exemplo.

Tomando o caso do primeiro exemplo citado, a densidade da distribuição gaussiana multivariada é descrita por,

$$
f_{\mathbf{x}}\left(x_{1}, \ldots, x_{n}\right)=(2 \pi)^{-\frac{n}{2}}|\boldsymbol{\Sigma}|^{-\frac{1}{2}} e^{-\frac{1}{2}(\mathbf{x}-\boldsymbol{\mu})^{\prime} \boldsymbol{\Sigma}^{-1}(\mathbf{x}-\boldsymbol{\mu})},
$$

onde $\mu \in \mathbb{R}^{n}$ e $\Sigma \in \mathbb{R}^{n \times n}$ é simétrica positiva definida. A variável $\boldsymbol{X}-\mu$ também pertence a mesma família de distribuição. Sua função característica é,

$$
\varphi_{\mathbf{X}-\mu}(\mathbf{t})=\exp \left(-\frac{1}{2} \mathbf{t}^{\prime} \boldsymbol{\Sigma} \mathbf{t}\right)
$$

Levando-se em conta a Definição 10, os respectivos parâmetros são $\mu, \Sigma$ e a função $\phi$ é dada por $\phi\left(\mathbf{t}^{\prime} \Sigma \mathbf{t}\right)=\exp \left\{\mathbf{t}^{\prime} \boldsymbol{\Sigma} \mathbf{t}\right\}$, o que mostra que $\boldsymbol{X}$ pertence a classe de distribuições elípticas. 
A cópula gaussiana bivariada é determinada nos mesmo moldes do Exemplo 1, tomando inicialmente a função de distribuição Normal bivariada e substituindo as variáveis pela sua inversa generalizada. Por esta razão, esta cópula é classificada como uma cópula elíptica. A cópula gaussiana tem a seguinte expressão,

$$
\begin{aligned}
C_{\rho}^{G a}\left(u_{1}, u_{2} ; \rho\right) & =\Phi_{G a}\left(\Phi^{-1}\left(u_{1}\right), \Phi^{-1}\left(u_{2}\right) ; \rho\right) \\
& =\int_{-\infty}^{\Phi^{-1}\left(u_{1}\right)} \int_{-\infty}^{\Phi^{-1}\left(u_{2}\right)} \frac{1}{2 \pi(1-\rho)^{1 / 2}} \times \exp \left\{\frac{-\left(s^{2}-2 \rho s t+t^{2}\right)}{2\left(1-\rho^{2}\right)}\right\} d s d t
\end{aligned}
$$

em que $\Phi$ é a distribuição acumulada da distribuição normal e o coeficiente de correlação linear de Pearson $\rho \in[0,1]$ é o parâmetro de dependência da cópula gaussiana. Esta cópula também é estendida para o caso multivariado $n \geq 3$ tomando como ponto de partida a distribuição normal multivariada $n$-dimensional.

A densidade da cópula gaussiana é dada por,

$$
c_{\rho}^{G a}\left(u_{1}, u_{2} ; \rho\right)=\frac{1}{|\Sigma|^{1 / 2}} \exp \left\{-\frac{1}{2} \psi^{\prime}\left(\Sigma^{-1}-\boldsymbol{I}\right) \psi\right\}
$$

em que $\psi=\left(\Psi^{-1}\left(u_{1}\right), \Psi^{-1}\left(u_{2}\right)\right)^{\prime}$ e $\Sigma$ é a matriz de correlação entre $U_{1}$ e $U_{2}$.

No gráfico abaixo temos as curvas de nível da função cópula e de sua densidade com parâmetro de dependência $\rho=0.6$ e com marginais normais padrão. Por meio da Figura 1.4 é possível examinar melhor a estrutura de dependência e sua forma elíptica. 


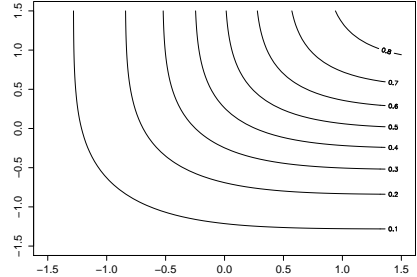

(a) $C_{\rho}^{G a}\left(u_{1}, u_{2} ; \rho=0,6\right)$

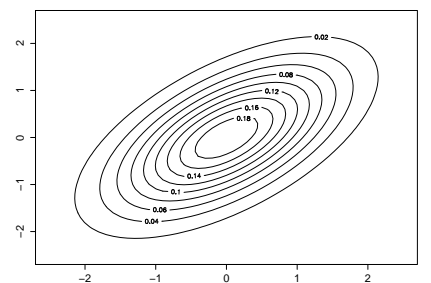

(b) $c_{\rho}^{G a}\left(u_{1}, u_{2} ; \rho=0,6\right)$

Figura 1.4: Curvas de nível - cópula Gaussiana $\rho=0,6$

No Gráfico 1.4b conseguimos observar uma estrutura de dependência linear que é captada pelo coeficiente de Pearson $\operatorname{com} \rho=0,6$. Entretanto, podemos medir a associação entre as variáveis com outras métricas discutidas na seção anterior. No caso da cópula gaussiana, há uma relação entre as medidas de $\tau$ de Kendall e o $\rho_{S}$ de Spearman com a correlação de Pearson $(\rho)$, expressas respectivamente abaixo,

$$
\begin{aligned}
\tau\left(C_{\rho}^{G a}\right) & =\frac{2}{\pi} \arcsin (\rho), \\
\rho_{S}\left(C_{\rho}^{G a}\right) & =\frac{6}{\pi} \arcsin \left(\frac{\rho}{2}\right) .
\end{aligned}
$$

Já o coeficiente de dependência caudal é zero, tanto para a cauda superior e inferior. Este resultado pode ser diretamente obtido utilizando a expressão de $\lambda_{U}$ dado pela equação (1.29),

$$
\begin{aligned}
\lim _{u \rightarrow 1^{-}} P\left[U_{2}>u \mid U_{1}=u\right] & =\lim _{x \rightarrow \infty} P\left[\Phi^{-1}\left(U_{2}\right)>x \mid \Phi^{-1}\left(U_{1}\right)=x\right] \\
& =\lim _{x \rightarrow \infty} P\left[X_{1}>x \mid X_{2}=x\right] \\
& =2 \lim _{x \rightarrow \infty} \bar{\Phi}\left(\frac{(x-\rho x)}{\sqrt{1-\rho^{2}}}\right) \\
& =2 \bar{\Phi}\left(\sqrt{\frac{1-\rho}{1+\rho}} \lim _{x \rightarrow \infty} x\right) .
\end{aligned}
$$


Note que se o vetor aleatório $\left(X_{1}, X_{2}\right)$ possui distribuição normal bivariada, então $X_{2} \mid X_{1}=x \sim N\left(\rho x, 1-\rho^{2}\right)$ e que a função $\Phi$ contínua tem $\lim _{x \rightarrow \infty} \bar{\Phi}(x)=$ $\lim _{x \rightarrow \infty} 1-\Phi(x)=0$. Desta forma, para $\rho<1$ temos que $\lambda_{U}=0$. O mesmo procede para a cauda inferior, portanto, $\lambda_{L}=0$.

Para gerar um vetor de variáveis aleatórias tomaremos como base o seguinte resultado já conhecido na literatura,

$$
\mu+A \boldsymbol{Z} \sim N(\mu, \Sigma)
$$

em que $\mu \in \mathbb{R}^{n}, \Sigma=A A^{\prime}$ é a decomposição de Cholesky, $Z_{1}, Z_{2}, \ldots, Z_{n} \sim N(0,1)$ e são independentes. O algoritmo que realiza a simulação de uma cópula gaussiana $n$-dimensional possui as seguintes etapas.

\section{Algoritmo 3 (Simulação: Cópula Gaussiana)}

1 Encontre a decomposição $\Sigma=A A^{\prime}$.

Para cada $i=1,2, \ldots, M$ execute

1 Simule $n$ variáveis aleatórias independentes $z_{1}, z_{2}, \ldots, z_{n} \sim N(0,1)$.

2 Compute $x=A z$.

3 Para cada $k=1,2, \ldots, n$ execute

$1 u_{k}=\Phi\left(x_{k}\right)$

4 Registre o i-ésimo vetor amostrado por $\left(u_{1}, u_{2}, \ldots, u_{n}\right)_{(i)}^{\prime}$.

$$
\left(u_{1}, u_{2}, \ldots, u_{n}\right)^{\prime} \sim C_{\Sigma}^{G a}
$$

Outra cópula elíptica bem conhecida é a t-Student. Da mesma maneira que a cópula gaussiana é proveniente da distribuição Normal multivariada, a cópula 
t-Student ou cópula-t é derivada da distribuição t-Student multivariada. A distribuição t-Student $\left(t_{\nu}\right)$ está relacionada com a distribuição normal multivariada nos mesmos moldes que o caso univariado. Sejam $Z$ e $S$ independentes tais que

$$
\boldsymbol{X} \stackrel{d}{=} \mu+\sqrt{\frac{\nu}{S}} \boldsymbol{Z}
$$

onde $\mu \in \mathbb{R}^{n}, S \sim \chi_{\nu}^{2}$ e $\boldsymbol{Z} \sim N(\mathbf{0}, \Sigma)$ Nessa situação $\boldsymbol{X}$ tem distribuição tStudent multivariada $n$-dimensional com $\nu$ graus de liberdade e é denotada por $\boldsymbol{X} \sim t(\mu, \nu, \Sigma) . \quad$ No caso em que $\nu>1, \mathbb{E}[\boldsymbol{X}]=\mu$ e para $\nu>2$ tem-se que $\operatorname{Var}[\boldsymbol{X}]=\frac{\nu}{\nu-2} \Sigma$. Tomando como base a distribuição t-Student multivariada, a formulação da cópula-t é escrita por,

$$
\begin{aligned}
C_{\nu, \Sigma}^{t}\left(u_{1}, u_{2} ; \rho, \nu\right) & =t_{\rho, \nu}\left(t_{\nu}^{-1}\left(u_{1}\right), t_{\nu}^{-1}\left(u_{2}\right)\right) \\
& =\int_{-\infty}^{t_{\nu}^{-1}\left(u_{1}\right)} \int_{-\infty}^{t_{\nu}^{-1}\left(u_{2}\right)} \frac{\Gamma\left(\frac{\nu+2}{2}\right)}{\Gamma\left(\frac{\nu}{2}\right)} \pi \nu \sqrt{1-\rho^{2}}\left(1+\frac{\psi^{\prime} \Sigma^{-1} \psi}{\nu}\right)^{-\frac{\nu+2}{2}} d \psi
\end{aligned}
$$

em que $\psi=\left(t_{\nu}^{-1}\left(u_{1}\right), t_{\nu}^{-1}\left(u_{2}\right)\right)^{\prime}$ e $\Gamma(\nu)$ é a função gama, o qual é escrita em termos de integral por,

$$
\Gamma(\nu)=\int_{0}^{\infty} x^{\nu-1} e^{-x} d x
$$

Da mesma forma que a cópula gaussiana, a cópula-t também permite estender para o caso multivariado em que $n \geq 3$, tomando como ponto de partida a distribuição t-Student multivariada $n$-dimensional. A densidade da cópula-t, é dada por,

$$
c_{\rho}^{t}\left(u_{1}, u_{2} ; \rho, \nu\right)=\frac{1}{|\Sigma|^{1 / 2}} \frac{\Gamma\left(\frac{\nu+2}{2}\right) \Gamma\left(\frac{\nu}{2}\right)}{\left[\Gamma\left(\frac{\nu+1}{2}\right)\right]^{2}} \frac{\left(1+\frac{1}{\nu} \psi^{\prime} \Sigma^{1} \psi\right)^{-\frac{\nu+2}{2}}}{\prod_{i=1}^{2}\left(1+\frac{1}{\nu} \psi_{i}^{2}\right)^{-\frac{\nu+1}{2}}} .
$$

Assim como a cópula gaussiana, há uma relação entre o $\tau$ de Kendall e a correlação 
de Pearson $\rho$ para a cópula-t, sendo expressa por,

$$
\tau\left(C_{\rho}\right)=\frac{2}{\pi} \arcsin (\rho)
$$

Já para a relação envolvendo o $\rho_{S}$ de Spearman não há expressão analítica, sendo necessário computá-lo numericamente (Jondeau et al., 2007).

Análogo ao que ocorre na distribuição normal bivariada, se $\left(X_{1}, X_{2}\right)$ tem distribuição t-Student bivariada com $\nu$ graus de liberdade e matriz de correlação $\Sigma$, então $X_{2} \mid X_{1}=x$ também tem distribuição t-Student com $\nu+1$ graus de liberdade e apresenta,

$$
\begin{aligned}
\mathbb{E}\left[X_{2} \mid X_{1}=x\right] & =\Sigma x \\
\operatorname{Var}\left[X_{2} \mid X_{1}=x\right] & =\left(\frac{\nu+x^{2}}{\nu+1}\right)\left(1-\rho^{2}\right) .
\end{aligned}
$$

Posto isso, podemos computar a dependência caudal de maneira mais simples através da equação (1.29), tal como foi feito para a cópula gaussiana, pois também possui simetria. Calculando para a cauda superior,

$$
\begin{aligned}
\lambda_{U} & =2 \lim _{u \rightarrow 1^{-}} P\left[U_{2}>u \mid U_{1}=u\right], \\
& =2 \lim _{x \rightarrow \infty} P\left[t_{\nu}\left(U_{2}\right)^{-1}>x \mid t_{\nu}\left(U_{1}\right)^{-1}=x\right]=2 \lim _{x \rightarrow \infty} P\left[X_{2}>x \mid X_{1}=x\right], \\
& =2 \lim _{x \rightarrow \infty} \bar{t}_{\nu+1}\left(\left(\frac{\nu+1}{\nu+x^{2}}\right)^{1 / 2} \frac{x-\rho x}{\sqrt{1-\rho^{2}}}\right), \\
& =2 \bar{t}_{\nu+1}\left(\sqrt{\frac{1-\rho}{1+\rho}}\left(\lim _{x \rightarrow \infty} \frac{\nu+1}{\nu / x^{2}+1}\right)^{1 / 2}\right), \\
& =2 \bar{t}_{\nu+1}\left(\sqrt{\frac{1-\rho}{1+\rho}} \sqrt{\nu+1}\right) .
\end{aligned}
$$

Diferentemente da cópula gaussiana, o coeficiente de dependência caudal da cópula-t não é igual a zero, salvo quando $\nu \rightarrow \infty$ onde neste caso a cópula-t 
converge em distribuição para a cópula gaussiana quando $\rho<1$. O coeficiente caudal aumenta conforme aumenta a correlação $\rho$ entre as variáveis. No sentido contrário, $\lambda$ é decrescente com relação a $\nu$.

Novamente, no gráfico abaixo, temos as curvas de nível da função cópula-t e de sua densidade com parâmetros de dependência $\rho=0.6$ e $\nu=3$. As marginais de $\left(X_{1}, X_{2}\right)$ possuem distribuições normais padrão. Por meio da Figura 1.5 é possível examinar o comportamento da dependência e sua forma linear.
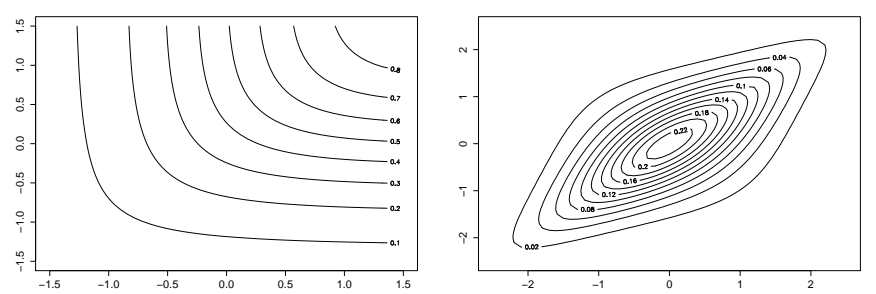

(a) $C_{\rho, \nu}^{t}\left(u_{1}, u_{2} ; \rho=(\mathrm{b}) \quad c_{\rho, \nu}^{t}\left(u_{1}, u_{2} ; \rho=\right.\right.$
$0.6, \nu=3)$
$0.6, \nu=3)$

Figura 1.5: Curvas de nível - Cópula-t $\rho=0,6$ e $\nu=3$

Através do comparativo entre os Gráficos 1.4b e 1.5b fica visível a diferença entre as formas das cópulas.

O algoritmo que realiza a simulação de uma cópula-t $n$-dimensional possui as seguintes etapas.

\section{Algoritmo 4 (Simulação: Cópula-t)}

1 Encontre a decomposição $\Sigma=A A^{\prime}$.

Para cada $i=1,2, \ldots, M$ execute

1 Simule $n$ variáveis aleatórias independentes $z_{1}, z_{2}, \ldots, z_{n} \sim N(0,1)$.

2 Simule a variável aleatória $s \sim \chi_{\nu}^{2}$ independentes de $z_{1}, z_{2}, \ldots, z_{n} \sim$ $N(0,1)$. 
3 Compute $\boldsymbol{y}=A \boldsymbol{z}$.

4 Compute $\boldsymbol{x}=\sqrt{\frac{\nu}{s}} \boldsymbol{y}$.

5 Para cada $k=1,2, \ldots, n$ execute

$1 u_{k}=t_{\nu}^{-1}\left(x_{k}\right)$

6 Registre o i-ésimo vetor amostrado por $\left(u_{1}, u_{2}, \ldots, u_{n}\right)_{(i)}^{\prime}$.

$$
\left(u_{1}, u_{2}, \ldots, u_{n}\right)^{\prime} \sim C_{\nu, \Sigma}^{t}
$$

Nas Figuras 1.6 e 1.7 são exibidos os resultados de uma simulação com 1000 amostras com cópula gaussiana e cópula-t, todos com marginais normais padrão. Para a primeira foram utilizados valores diferentes para o parâmetro $\rho$. Na segunda também foram usados os mesmos valores para o parâmetro $\rho$, porém com $\nu=3$ graus de liberdade para todos os casos.

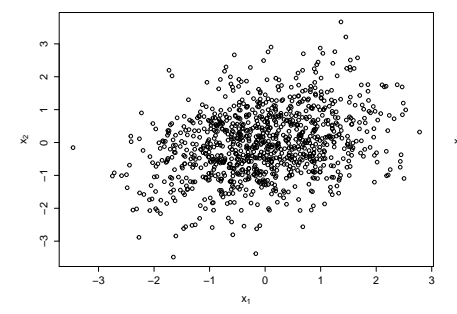

(a) $\rho_{1}=0.3$

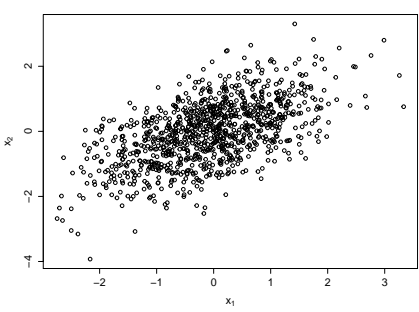

(b) $\rho_{2}=0.6$

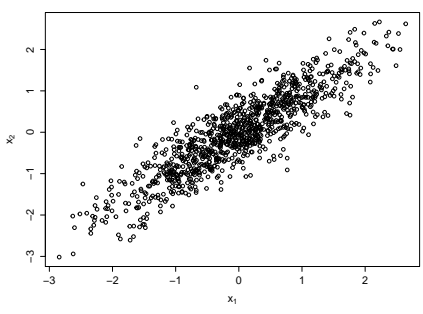

(c) $\rho_{3}=0.9$

Figura 1.6: Dispersão $c_{\rho_{i}}^{G a}\left(F\left(x_{1}\right), F\left(x_{2}\right)\right), i=1,2,3$, marginal normal padrão 


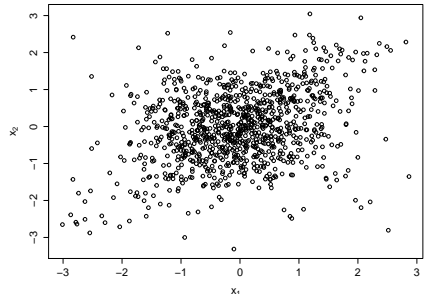

(a) $\rho_{1}=0.3, \nu=3$

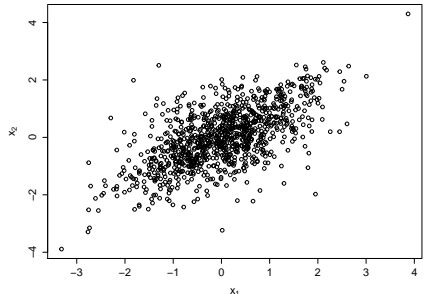

(b) $\rho_{2}=0.6, \nu=3$

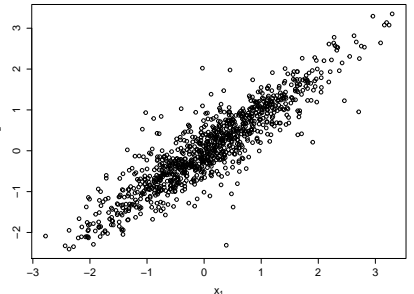

(c) $\rho_{3}=0.9, \nu=3$

Figura 1.7: Dispersão $c_{\rho_{i}, \nu}^{t}\left(F\left(x_{1}\right), F\left(x_{2}\right)\right), i=1,2,3$, marginal normal padrão

As cópulas elípticas possuem a desvantagem de não possuírem uma expressão de forma fechada e estão restrita a simetria radial. Todavia, em diversas aplicações, como em finanças não se observa tal comportamento. No mercado financeiro, os estudos empíricos mostram que há uma maior associação entre perdas do que ganhos entre mercados ou ativos (Embrechts et al., 2001a). Para captar uma estrutura assimétrica de dependência é necessário lançar mão de outra família de cópulas. Uma classe importante de famílias de cópulas que possui uma grande variedade de estrutura de dependência é a arquimediana, que será apresentada na próxima seção.

\subsubsection{Cópulas Arquimedianas}

A classe de cópulas chamadas de Arquimedianas é amplamente utilizada em aplicações empíricas e largamente debatida na literatura. Sua popularidade se deve em razão da gama de estrutura de dependência (Trivedi e Zimmer, 2005). Outras razões para isso é a facilidade da construção de suas expressões, diversidade de famílias pertencentes a essa classe, além de algumas propriedades interessantes (Nelsen, 2006).

Diferentemente das cópulas elípticas, as cópulas arquimedianas não são construídas a partir de distribuições multivariadas seguindo o Teorema de Sklar. Além 
disso, usualmente possuem expressões analíticas fechadas, o que trás algumas vantagens para computar seus valores. Sua construção é feita por meio de uma função geradora que será definida mais adiante.

Definição 11 (Pseudo-Inversa de $\varphi$ ). Seja $\varphi: I \rightarrow[0, \infty]$ uma função contínua e estritamente decrescente tal que $\varphi(1)=0$. A pseudo inversa de $\varphi$ é a função $\varphi^{[-1]} \operatorname{com} \operatorname{Dom} \varphi^{[-1]}=[0, \infty]$ e $\operatorname{Ran} \varphi^{[-1]}=\boldsymbol{I}$ dada por,

$$
\varphi^{[-1]}(t)= \begin{cases}\varphi^{-1}(t), & \text { se } 0 \leq t \leq \varphi(0), \\ 0, & \text { se } \varphi(0) \leq t<\infty\end{cases}
$$

Com isso, perceba que $\varphi^{[-1]}$ é contínua e não crescente em $[0, \infty)$, e estritamente decrescente em $[0, \varphi(0)]$. Adicionalmente, $\varphi^{[-1]}(\varphi(u))=u \in \boldsymbol{I}$ e,

$$
\begin{aligned}
\varphi\left(\varphi^{[-1]}(t)\right) & = \begin{cases}t, & \text { se } 0 \leq t \leq \varphi(0) \\
\varphi(0), & \text { se } \varphi(0) \leq t<\infty\end{cases} \\
& =\min \{t, \varphi(0)\}
\end{aligned}
$$

Por último, se $\varphi(0)=\infty$, então $\varphi^{[-1]}=\varphi^{-1}$.

Teorema 6 Seja $\varphi:[0,1] \rightarrow[0, \infty]$ uma função contínua e estritamente decrescente em que $\varphi(1)=0$ e $\varphi^{[-1]}$ a pseudo-inversa de $\varphi$. Seja também uma função $C$ : $[0,1]^{2} \rightarrow[0,1]$ dada por

$$
C\left(u_{1}, u_{2}\right)=\varphi^{[-1]}\left(\varphi\left(u_{1}\right)+\varphi\left(u_{2}\right)\right)
$$

então $C$ é uma cópula se e somente se $\varphi$ for convexa.

A prova para o teorema pode ser vista em Nelsen (2006) p.111.

As cópulas com a estrutura apresentada na equação (1.39) são denominadas de Arquimedianas. Como já citado anteriormente, a construção das cópulas 
Arquimedianas se dá através da função $\varphi$, denominada de função geradora. Se $\varphi(0)=\infty$, isto é, $\varphi^{[-1]}=\varphi^{-1}$, então $\varphi$ é denominada de função geradora estrita. Assim, esta classe de cópulas é chamada de Arquimediana estrita. A densidade associada a uma cópula Arquimediana bivariada (1.39) pode ser encontrada tomando $\varphi\left(C\left(u_{1}, u_{2}\right)\right)$ e diferenciando $C$ com relação a $u_{1}$ e posteriormente a $u_{2}$, com isto temos que,

$$
\varphi^{\prime}\left(C\left(u_{1}, u_{2}\right)\right) \frac{\partial C\left(u_{1}, u_{2}\right)}{\partial u_{1}}=\varphi^{\prime}\left(u_{1}\right)
$$

e

$$
\varphi^{\prime \prime}\left(C\left(u_{1}, u_{2}\right)\right) \frac{\partial C\left(u_{1}, u_{2}\right)}{\partial u_{1}} \frac{\partial C\left(u_{1}, u_{2}\right)}{\partial u_{2}}+\varphi^{\prime}\left(C\left(u_{1}, u_{2}\right)\right) \frac{\partial^{2} C\left(u_{1}, u_{2}\right)}{\partial u_{1} \partial u_{2}}=0,
$$

$\operatorname{logo}$, isolando $\frac{\partial^{2} C}{\partial u_{1} \partial u_{2}}$,

$$
c\left(u_{1}, u_{2}\right)=-\frac{\varphi^{\prime \prime}\left(C\left(u_{1}, u_{2}\right)\right) \varphi^{\prime}\left(u_{1}\right) \varphi^{\prime}\left(u_{2}\right)}{\left[\varphi^{\prime}\left(C\left(u_{1}, u_{2}\right)\right)\right]^{3}} .
$$

A distribuição condicional das cópulas Arquimedianas é,

$$
D_{1} C\left(u_{1}, u_{2}\right)=\frac{\varphi^{\prime}\left(u_{1}\right)}{\varphi^{\prime}\left(\varphi^{-1}\left(\varphi\left(u_{1}\right)+\varphi\left(u_{2}\right)\right)\right)} .
$$

Em geral, as derivadas não existem nas bordas, isto é, $\varphi\left(u_{1}\right)+\varphi\left(u_{2}\right)=\varphi(0)$. Mais detalhes podem ser visto em Genest e MacKay (1986).

Como dito no início desta seção, esta classe de cópulas possui propriedades interessantes, sendo algumas delas descritas na proposição abaixo.

Proposição 1 (Propriedades Cópulas Arquimedianas). Seja $C$ uma cópula Arquimediana e $\varphi$ sua função geradora, então,

1. $C$ é simétrico, ou seja, $C\left(u_{1}, u_{2}\right)=C\left(u_{2}, u_{1}\right) \forall\left(u_{1}, u_{2}\right) \in \boldsymbol{I}^{2}$.

2. $C$ é associativo, isto é, $C\left(C\left(u_{1}, u_{2}\right), u_{3}\right)=C\left(u_{1}, C\left(u_{2}, u_{3}\right)\right)$.

3. Se $k>0$ é uma constante, então $k \varphi$ também é uma geradora de $C$. 
Prova. Para a 1 é imediata de (1.39). A prova para 2 segue abaixo a partir da definição,

$$
\begin{aligned}
C\left(C\left(u_{1}, u_{2}\right), u_{3}\right) & =\varphi^{[-1]}\left(\varphi\left(\varphi^{[-1]}\left(\varphi\left(u_{1}\right)+\varphi\left(u_{2}\right)\right)\right)+\varphi\left(u_{3}\right)\right) \\
& =\varphi^{[-1]}\left(\varphi\left(u_{1}\right)+\varphi\left(u_{2}\right)+\varphi\left(u_{3}\right)\right) \\
& \varphi\left(\varphi\left(u_{1}\right)+\varphi\left(\varphi^{[-1]}\left(\varphi\left(u_{1}\right)+\varphi\left(u_{2}\right)\right)\right)\right) \\
& =C\left(u_{1}, C\left(u_{2}, u_{3}\right)\right) .
\end{aligned}
$$

Por último, se $\varphi$ é uma função convexa e estritamente decrescente, então com $k>0$ a função $\varphi^{*}=k \varphi$ também é, visto que se $\frac{d \varphi}{d t}<0 \Rightarrow \frac{d k \varphi}{d t}=k \frac{d \varphi}{d t}<0$ e se $\frac{d^{2} \varphi}{d t^{2}}>$ $0 \Rightarrow \frac{d^{2} k \varphi}{d t^{2}}=k \frac{d^{2} \varphi}{d t^{2}}>0$. Note também que se $\varphi(1)=0$, então $\varphi^{*}(1)=k \varphi(1)=0$. Tomando a inversa tem-se que $\varphi^{[-1]}(t / k)=\varphi^{*[-1]}(t) \operatorname{logo}$

$$
\begin{aligned}
C\left(u_{1}, u_{2}\right) & =\varphi^{*[-1]}\left(\varphi^{*}\left(u_{1}\right)+\varphi^{*}\left(u_{2}\right)\right), \\
& =\varphi^{*[-1]}\left(k \varphi\left(u_{1}\right)+k \varphi\left(u_{2}\right)\right), \\
& \varphi^{[-1]}\left(\frac{1}{k}\left(k \varphi\left(u_{1}\right)+k \varphi\left(u_{2}\right)\right)\right), \\
& =\varphi^{[-1]}\left(\left(\varphi\left(u_{1}\right)+\varphi\left(u_{2}\right)\right)\right) .
\end{aligned}
$$

Como visto anteriormente, podemos obter a distribuição acumulada condicional derivando a função cópula em uma das variáveis,

$$
P\left[U_{2}<u_{2} \mid U_{1}=u_{1}\right]=D_{1} C\left(u_{1}, u_{2}\right)=\frac{\varphi^{\prime}\left(u_{1}\right)}{\varphi^{\prime}\left(C\left(u_{1}, u_{2}\right)\right)}
$$

Teorema 7 Seja $C$ uma função cópula Arquimediana com função geradora $\varphi$ e seja

$$
K_{C}(t)=\operatorname{Vol}_{C}\left(\left\{\left(u_{1}, u_{2}\right) \in \boldsymbol{I}^{2} \mid C\left(u_{1}, u_{2}\right) \leq t\right\}\right)
$$


então é possível computar para $t \in[0,1]$ seu valor por,

$$
K_{C}(t)=t-\frac{\varphi(t)}{\varphi^{\prime}\left(t^{+}\right)}
$$

A prova pode ser vista em Nelsen (2006) p.127.

Corolário 2 Se $\left(U_{1}, U_{2}\right)^{\prime}$ possui uma função cópula arquimediana $C$ com função geradora $\varphi$, então a função $K_{C}$ do teorema acima dado por (1.43) é a função de distribuição da variável aleatória $T=C\left(U_{1}, U_{2}\right)$.

A partir dos resultados apresentados acima, o cálculo de medidas de dependência torna-se mais simples de ser avaliado. No caso da medida de $\tau$ de Kendall, ela é computada por uma integral dupla como definida na equação (1.24), o que pode ser complicado para alguns casos. Todavia, para as cópulas Arquimedianas essa medida fica mais fácil de ser avaliada, visto que ela pode ser escrita por uma integral simples. O teorema abaixo mostra como é computado a correlação $\tau$ de Kendall.

Teorema 8 Sejam $X_{1}$ e $X_{2}$ variáveis aleatórias com uma estrutura de dependência dada pela cópula Arquimediana $C$ e sua respectiva função geradora $\varphi$, a medida de $\tau$ de Kendall de $X_{1}$ e $X_{2}$ é dada por,

$$
\tau_{C}=\tau\left(X_{1}, X_{2}\right)=1+4 \int_{0}^{1} \frac{\varphi(t)}{\varphi^{\prime}(t)} d t
$$

onde $\varphi^{\prime}(t)=\frac{d \varphi(t)}{d t}$.

Prova. Sejam as variáveis aleatórias $U_{1}, U_{2} \sim U(0,1)$ e com distribuição conjunta dada pela cópula Arquimediana $C$ e $K_{C}$ a função de distribuição de $T=C\left(U_{1}, U_{2}\right)$, 
então da equação (1.25) obtemos,

$$
\begin{aligned}
\tau_{C}=\tau\left(X_{1}, X_{2}\right) & =4 \mathbb{E}\left[C\left(U_{1}, U_{2}\right)\right]-1=4 \int_{0}^{1} t d K_{C}(t)-1 \\
& =4\left(\left.t K_{C}(t)\right|_{0} ^{1}-\int_{0}^{1} K_{C}(t) d t\right)-1=3-4 \int_{0}^{1} K_{C}(t) d t
\end{aligned}
$$

em que no segundo passo toma-se a integral por partes ${ }^{1}$ e utilizando a equação (1.43) temos finalmente que,

$$
\tau_{C}=1+4 \int_{0}^{1} \frac{\varphi(t)}{\varphi^{\prime}(t)} .
$$

Da mesma forma que ocorre para o $\tau$ de Kendall, o coeficiente de dependência caudal de um cópula Arquimediana também pode ser expresso em termos de sua função geradora. Seguindo a notação de Nelsen (2006), vamos denotar por $\Omega$ o conjunto de funções contínuas estritamente decrescente e convexa tal que $\varphi$ : $[0,1] \rightarrow[0, \infty]$ e $\varphi(1)=0$, isto é, funções geradoras estritas.

Teorema 9 Seja $C$ uma cópula Arquimediana com função geradora $\varphi \in \Omega$, então,

$$
\begin{aligned}
& \lambda_{U}=2-\lim _{t \rightarrow 1^{-}} \frac{1-\varphi^{[-1]}(2 \varphi(t))}{1-t}=2-\lim _{x \rightarrow 0^{+}} \frac{1-\varphi^{[-1]}(2 x)}{1-\varphi^{[-1]}(x)}=2-2 \lim _{t \rightarrow 0^{+}} \frac{\varphi^{-1 \prime}(2 t)}{\varphi^{-1 \prime}(t)} \\
& \lambda_{L}=\lim _{t \rightarrow 0^{+}} \frac{\varphi^{[-1]}(2 \varphi(t))}{t}=\lim _{x \rightarrow \infty} \frac{\varphi^{[-1]}(2 x)}{\varphi^{[-1]}(x)}=2 \lim _{t \rightarrow \infty} \frac{\varphi^{-1 \prime}(2 t)}{\varphi^{-1 \prime}(t)} .
\end{aligned}
$$

Prova. Considere inicialmente $\psi=\varphi^{\prime}$. Para o caso de $\lambda_{U}$, temos a partir da

\footnotetext{
${ }^{1}$ Integral por partes: $\int_{a}^{b} g(t) h^{\prime}(t) d t=\left.g(t) h(t)\right|_{a} ^{b}-\int_{a}^{b} g^{\prime}(t) h(t) d t$ $g(t)=t$ e $h^{\prime}(t) d t=d K_{C}(t) \Rightarrow h(t)=K_{C}(t)$ e $g^{\prime}(t)=1$
} 
equação (1.26),

$$
\begin{aligned}
\lambda_{U} & =\lim _{u \rightarrow 1^{-}} \frac{\bar{C}(u, u)}{1-u}=-\lim _{u \rightarrow 1^{-}} \frac{d \bar{C}(u, u)}{d u} \\
& =-\lim _{u \rightarrow 1^{-}} \frac{d}{d u}\left(1-2 u+\psi\left(2 \psi^{-1}(u)\right)\right)= \\
& =-\lim _{u \rightarrow 1^{-}}\left(-2+\psi^{\prime}\left(2 \psi^{-1}\right) 2 \psi^{-1 \prime}(u)\right) \\
& =2-2 \lim _{u \rightarrow 1^{-}} \frac{\psi^{\prime}\left(2 \psi^{-1}(u)\right)}{\psi^{\prime}\left(\psi^{-1}(u)\right)}=2-2 \lim _{u \rightarrow 1^{-}} \frac{\varphi^{-1 \prime}(2 \varphi(u))}{\varphi^{-1 \prime}(\varphi(u))} \\
& =2-2 \lim _{t \rightarrow 0^{+}} \frac{\varphi^{-1^{\prime}(2 t)}}{\varphi^{-1 \prime}(t)} .
\end{aligned}
$$

Note que na passagem da segunda linha (1.45b) para a terceira (1.45d) utilizamos a regra da diferenciação da inversa ${ }^{2}$ e na primeira linha (1.45a) o resultado da equação (1.28). Por último, foi substituído a variável $t=\varphi(u)$ em (1.45e). A prova para $\lambda_{L}$ segue a mesma construção.

$$
2\left[f^{-1}\right]^{\prime}(a)=\frac{1}{f^{\prime}\left(f^{-1}(a)\right)}
$$




\begin{tabular}{|c|c|c|c|c|c|}
\hline $\mathrm{N}^{\mathrm{O}}$ & Cópula & $C_{\theta}\left(u_{1}, u_{2}\right)$ & $\varphi_{\theta}(t)$ & $\theta \in$ & Limites e Casos Especiais \\
\hline 1 & Clayton & {$\left[\max \left(u_{1}^{-\theta}+u_{2}^{-\theta}-1,0\right)\right]^{-\frac{1}{\theta}}$} & $\frac{1}{\theta}\left(t^{-\theta}-1\right)$ & {$[-1, \infty) \backslash\{0\}$} & $\begin{array}{c}C_{-1}=W, C_{0}=\Pi \\
C_{\infty}=M\end{array}$ \\
\hline 2 & Joe & $1-\left[\left(1-u_{1}\right)^{\theta}+\left(1-u_{2}\right)^{\theta}-\left(1-u_{1}\right)^{\theta}\left(1-u_{2}\right)^{\theta}\right]^{\frac{1}{\theta}}$ & $-\ln \left(1-(1-t)^{t}\right.$ & $\left.{ }^{\theta}\right)[1, \infty)$ & $C_{1}=\Pi, C_{\infty}=M$ \\
\hline 3 & Frank & $-\frac{1}{\theta} \ln \left(1+\frac{\left(e^{-\theta u_{1}}-1\right)\left(e^{-\theta u_{2}}-1\right)}{e^{-\theta}-1}\right)$ & $-\ln \left(\frac{e^{-\theta t}-1}{e^{-\theta}-1}\right)$ & $(-\infty, \infty) \backslash\{0\}$ & $\begin{array}{c}C_{-\infty}=W, C_{0}=\Pi \\
C_{\infty}=M\end{array}$ \\
\hline 4 & Gumbel & $\exp \left\{-\left[\left(-\ln u_{1}\right)^{\theta}+\left(-\ln u_{2}\right)^{\theta}\right]^{\frac{1}{\theta}}\right\}$ & $(-\ln t)^{\theta}$ & {$[1, \infty)$} & $C_{1}=\Pi, C_{\infty}=M$ \\
\hline 5 & Gumbel-Barnett & $u_{1} u_{2} \exp \left\{-\theta \ln u_{1} \ln u_{2}\right\}$ & $\ln (1-\theta \ln t)$ & $(0,1]$ & $C_{0}=\Pi$ \\
\hline 6 & Ali-Mikhail-Haq & $\frac{u_{1} u_{2}}{1-\theta\left(1-u_{1}\right)\left(1-u_{2}\right)}$ & $\ln \left(\frac{1-\theta(1-t)}{t}\right)$ & {$[-1,1]$} & $C_{0}=\Pi$ \\
\hline
\end{tabular}

Tabela 1.1: Algumas famílias de cópulas Arquimedianas 
Para cada uma das cópulas Arquimedianas apresentadas na Tabela 1.1 é plotado o gráfico das curvas de nível da densidade da cópula para três valores distintos de $\theta$. Em todos os casos foram consideradas marginais com distribuição normal padrão.

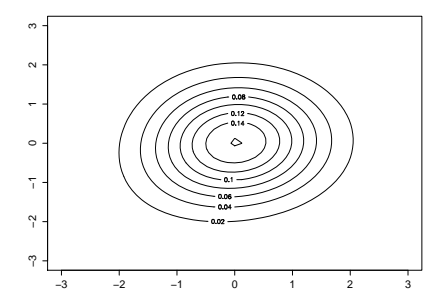

(a) $\theta=0.5$

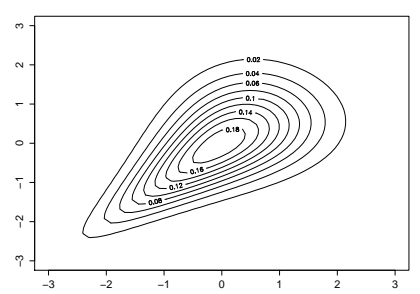

(b) $\theta=1.2$

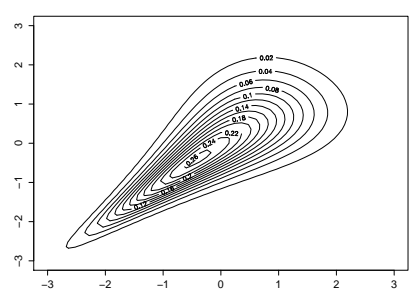

(c) $\theta=2.3$

Figura 1.8: Clayton $c_{\theta_{i}}\left(F\left(x_{1}\right), F\left(x_{2}\right)\right), i=1,2,3$, marginal normal padrão

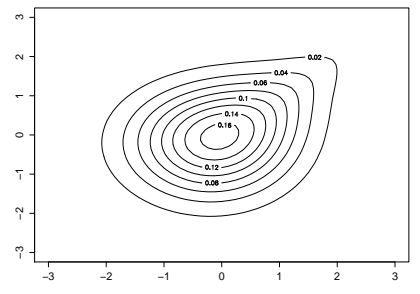

(a) $\theta=1.3$

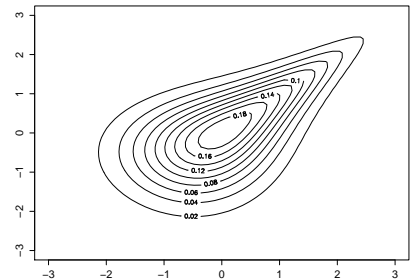

(b) $\theta=2$

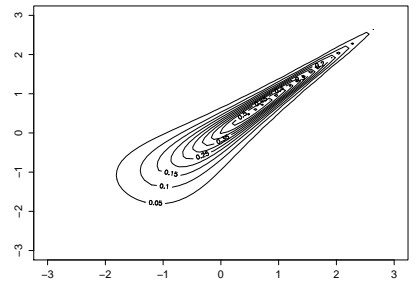

(c) $\theta=6$

Figura 1.9: Joe $c_{\theta_{i}}\left(F\left(x_{1}\right), F\left(x_{2}\right)\right), i=1,2,3$, marginal normal padrão

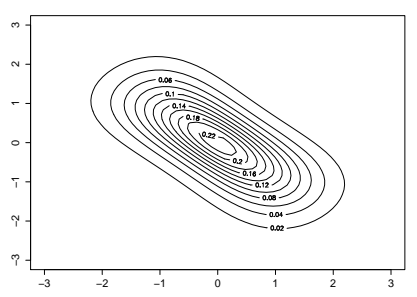

(a) $\theta=-5$

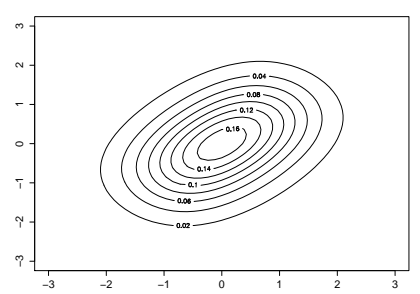

(b) $\theta=2.2$

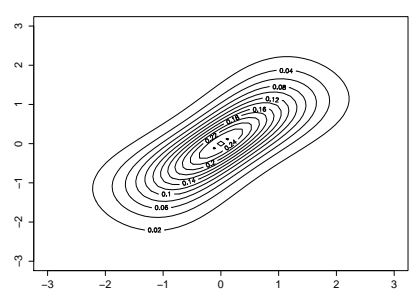

(c) $\theta=6$

Figura 1.10: Frank $c_{\theta_{i}}\left(F\left(x_{1}\right), F\left(x_{2}\right)\right), i=1,2,3$, marginal normal padrão 


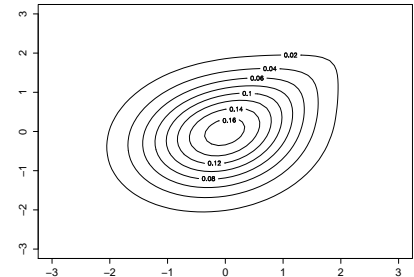

(a) $\theta=1.2$

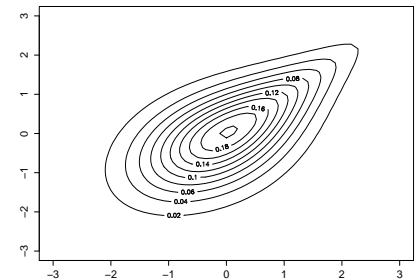

(b) $\theta=1.6$

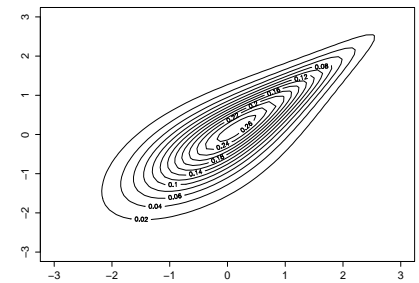

(c) $\theta=2.3$

Figura 1.11: Gumbel $c_{\theta_{i}}\left(F\left(x_{1}\right), F\left(x_{2}\right)\right), i=1,2,3$, marginal normal padrão

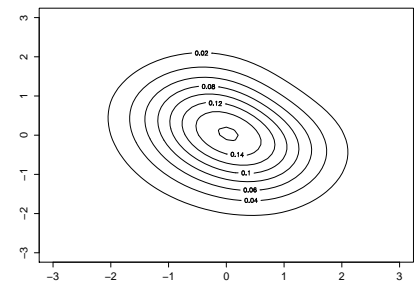

(a) $\theta=-0.8$

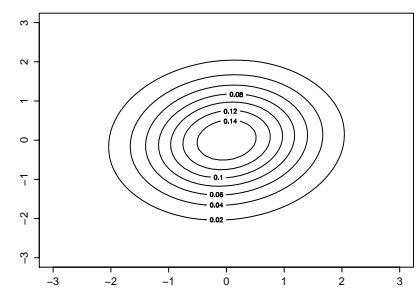

(b) $\theta=0.2$

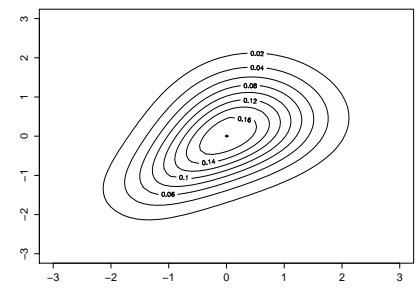

(c) $\theta=0.9$

Figura 1.12: Ali-Mikhail-Haq $c_{\theta_{i}}\left(F\left(x_{1}\right), F\left(x_{2}\right)\right), i=1,2,3$, marginal normal padrão 


\subsubsection{Cópulas de Valores Extremos}

$\mathrm{Na}$ análise de risco, é importante que os agentes analisem não apenas a região modal da distribuição dos dados mas também suas caudas. Nestas situações, estamos interessados em eventos extremos como a observação dos valores de máximo ou mínimo de um conjunto de dados (Jondeau et al., 2007). Esses casos ocorrem por exemplo na área de finanças, hidrologia e geofísica.

Para um conjunto de variáveis aleatórias $X_{1}, X_{2}, \ldots, X_{n}$ vamos denotar,

$$
M_{n} \equiv \max \left\{X_{1}, X_{2}, \ldots, X_{n}\right\}
$$

Devido a relação acima, é suficiente focar no máximo ou na cauda superior. Se $F_{X}$ é a distribuição acumulada de $X_{i}, i=1,2, \ldots, n$ e todas independentes e identicamente distribuídas, então segue que,

$$
\begin{aligned}
P\left[M_{n} \leq x\right] & =P\left[\max \left\{X_{1}, X_{2}, \ldots, X_{n}\right\} \leq x\right] \\
& =P\left[X_{1} \leq x, X_{2} \leq x, \ldots, X_{n} \leq x\right] \\
& =\prod_{i=1}^{n} P\left[X_{i} \leq x\right]=\left[F_{X}(x)\right]^{n}
\end{aligned}
$$

Uma questão que surge é se $M_{n}$ normalizado converge para alguma distribuição. Nesse sentido, o teorema conhecido como Fisher e Tippett (1928) caracteriza a distribuição limite.

Teorema 10 (Fisher-Tippett). Seja $X_{1}, X_{2}, \ldots, X_{n}$ uma sequência de variáveis aleatórias independentes e identicamente distribuídas com mesma distribuição $F$. Se existirem um parâmetro de locação $d_{n} \in \mathbb{R}$ e um de escala $c_{n}>0$ e para uma função não degenerada $H$, tal que o limite da distribuição padronizada do máximo $Y_{n}=\frac{M_{n}-d_{n}}{c_{n}}$ converge para $H$, isto é,

$$
\lim _{n \rightarrow \infty} \operatorname{Pr}\left(\frac{M_{n}-d_{n}}{c_{n}} \leq y\right)=H(y)
$$


então $H$ é um dos três tipos de função de distribuição a seguir:

Weibull $\Psi_{\alpha}(y)$

$$
\Psi_{\alpha>0}(y)= \begin{cases}\exp \left(-(-y)^{\alpha}\right), & \text { para } y \leq 0 \\ 1, & \text { para } y>0\end{cases}
$$

Gumbel $\Lambda_{\alpha}(y)$

$$
\Lambda_{\alpha}(y)=\exp (-\exp (-y)) \text { para } y \in \mathbb{R}
$$

Frechet $\Phi_{\alpha}(y)$

$$
\Phi_{\alpha>0}(y)= \begin{cases}0, & \text { para } y \leq 0 \\ \exp \left(-y^{-\alpha}\right), & \text { para } y>0\end{cases}
$$

No caso em que há a convergência dizemos que $F_{X}$ pertence ao domínio de atração de $H$ ou $F_{X} \in D(H)$. Uma forma de representar as três distribuições foi introduzida por Jenkinson (1955), através de uma única distribuição chamada Valores Extremos Generalizada GEV (Generalized Extreme Value).

Definição 12 (GEV). A distribuição generalizada de valor extremo não normalizada é dada por,

$$
H_{(n)}(x, y)= \begin{cases}\exp \left\{-\left(1+\xi \frac{y-\mu}{\sigma}\right)^{-1 / \xi}\right\}, & \text { se } \xi \neq 0 \text { e } 1+\xi \frac{y-\mu}{\sigma}>0 \\ \exp \left\{-\exp \left(-\frac{y-\mu}{\sigma}\right)\right\}, & \text { se } \xi=0 .\end{cases}
$$

em que $\mu$ é o parâmetro de locação e $\sigma$ o parâmetro de escala.

A relação entre as distribuições e a GEV é feita mediante ao parâmetro $\xi$. Se $\xi>0$, tem-se a Fréchet, se $\xi=0$ tem-se a Gumbel e por fim se $\xi<0$, obtém-se a distribuição Weibull.

Da equação (1.46) podemos realizar a mesma formulação para o caso bidimensional e, em seguida, escrever em termos da função cópula. Sejam os vetores de variáveis aleatórias $\left(X_{1}, Y_{1}\right),\left(X_{2}, Y_{2}\right), \ldots,\left(X_{n}, Y_{n}\right)$ independentes e identicamente 
distribuídos com função conjunta $H$ comum a todas, cópula $C$ e marginais $F_{X}$ e $F_{Y}$. Tomando $X_{(n)}=\max \left\{X_{i}\right\}, Y_{(n)}=\max \left\{Y_{i}\right\} i=1,2, \ldots, n$ e as suas respectivas marginais por $F_{X_{(n)}}=\left[F_{X}\right]^{n}$ e $F_{Y_{(n)}}=\left[F_{Y}\right]^{n}$ então a distribuição conjunta $H_{(n)}$ de $X_{(n)}$ e $Y_{(n)}$ pode ser escrita por,

$$
\begin{aligned}
H_{(n)}(x, y) & =P\left[X_{(n)} \leq x, Y_{(n)} \leq y\right]=P\left[\forall i=1,2, . ., n: X_{i} \leq x, Y_{i} \leq y\right] \\
& =[H(x, y)]^{n}=\left[C\left(F_{X}(x), F_{Y}(y)\right)\right]^{n} \\
& =\left[C\left(\left[F_{X_{(n)}}(x)\right]^{\frac{1}{n}},\left[F_{Y_{(n)}}(y)\right]^{\frac{1}{n}}\right)\right]^{n} .
\end{aligned}
$$

Assim, temos que,

$$
C_{(n)}\left(u_{1}, u_{2}\right)=C^{n}\left(u_{1}^{1 / n}, u_{2}^{1 / n}\right) \text { para }\left(u_{1}, u_{2}\right) \in \boldsymbol{I}^{2} .
$$

A partir dos resultados acima e do caso limite da equação (1.51), podemos introduzir duas definições.

Definição 13 (Max-Stable) Uma cópula $C$ é dita max-stable se $\forall r \in \mathbb{R}$ tem-se que,

$$
C\left(u_{1}, u_{2}\right)=C^{r}\left(u_{1}^{1 / n r}, u_{2}^{1 / n r}\right)
$$

$\operatorname{com}\left(u_{1}, u_{2}\right) \in \boldsymbol{I}^{2}$.

Definição 14 (Cópula de Valor Extremo) Uma função cópula $C_{*}$ é uma cópula de valor extremo se existe um cópula $C$ tal que,

$$
C_{*}\left(u_{1}, u_{2}\right)=\lim _{n \rightarrow \infty} C^{n}\left(u_{1}^{1 / n}, u_{2}^{1 / n}\right),
$$

para todo $\left(u_{1}, u_{2}\right) \in \boldsymbol{I}^{2}$. Neste caso dizemos que $C$ pertence ao domínio de atração de $C_{*}$ no qual será denotado por $C \in D\left(C_{*}\right)$.

Com estas duas formulações podemos perceber naturalmente que toda cópula do tipo max-stable é uma cópula de valor extremo. No sentido contrário, se $C_{*}$ é 
uma cópula de valor extremo, então ela é max-stable. Do limite (1.53) da definição é possível ver este resultado como segue,

$$
\begin{aligned}
C_{*}^{r}\left(u_{1}^{1 / r}, u_{2}^{1 / r}\right) & =\lim _{n \rightarrow \infty} C^{r n}\left(u_{1}^{1 / r}, u_{2}^{1 / r}\right) \\
& =C_{*}\left(u_{1}, u_{2}\right) .
\end{aligned}
$$

Um método para construir cópulas de valores extremos foi desenvolvido por Pickands (1981) por meio de uma formulação geral que pode ser vista através do seguinte teorema.

Teorema 11 Uma cópula bivariada $C$ é de valor extremo se, e somente se, puder ser escrita da seguinte forma,

$$
C\left(u_{1}, u_{2}\right)=\exp \left\{\ln \left(u_{1} u_{2}\right) A\left(\frac{\ln u_{1}}{\ln \left(u_{1} u_{2}\right)}\right)\right\},\left(u_{1}, u_{2}\right) \in(0,1]^{2} \backslash\{(1,1)\}
$$

em que a função $A:[0,1] \rightarrow[1 / 2,1]$ é convexa e satisfaz $\max \{t, 1-t\} \leq A(t) \leq 1$ $\forall t \in[0,1]$.

A função $A$ é denominada de função de dependência de Pickands. Observe que se $A(t)=1$ gera a cópula $\Pi$. No lado oposto, se $A(t)=\max \{t, 1-t\}$, a cópula será $M$.

Exemplo 2 (Nelsen, 2005). Definindo $A(t)=\left(t^{\theta}+(1-t)^{\theta}\right)^{1 / \theta}$, para $\theta>1$ teremos a cópula a de Gumbel-Hougaard descrita na Tabela 1.1 linha de número 4. Logo, a cópula Arquimediana de Gumbel-Hougaard é de valor extremo. Se por outro lado definirmos $A(t)=1-\min \{\beta t, \alpha(1-t)\}$ para $\beta, \alpha \in \boldsymbol{I}$ temos a cópula de Marshall-Olkin expressa por,

$$
C_{\alpha, \beta}\left(u_{1}, u_{2}\right)=\min \left(u_{1}^{1-\alpha} u_{2}, u_{1} u_{2}^{1-\beta}\right)= \begin{cases}u_{1}^{1-\alpha} u_{2}, & \text { se } u_{1}^{\alpha} \geq u_{2}^{\beta}, \\ u_{1} u_{2}^{1-\beta}, & \text { se } u_{1}^{\alpha} \leq u_{2}^{\beta},\end{cases}
$$

em que $\alpha$ e $\beta$ são os parâmetros de dependência. Sendo assim, a cópula de Marshall-Olkin também é de valores extremos. 
Assim como para as cópulas Arquimedianas, as cópulas de valores extremos também possuem a vantagem de permitir calcular algumas medidas de dependência por meio da função que as caracterizam. No caso das cópulas de valores extremo, esta função é $A(t)$ e portanto os coeficientes de dependência para esta classe de cópulas ficam em função dela. Para o coeficiente de $\tau$ de Kendall, seu valor é computado pela fórmula,

$$
\tau_{C}=\int_{0}^{1} \frac{t(1-t)}{A(t)} A^{\prime \prime}(t) d t
$$

No caso do coeficiente de $\rho_{S}$ de Spearman temos a seguinte expressão,

$$
\rho_{S}=12 \int_{0}^{1}[A(t)+1]^{-2} d t-3
$$

É possível também descrever uma relação entre o coeficiente de dependência caudal e a função $A(t)$. Para a dependência caudal superior,

$$
\lambda_{U}=2\left[1-A\left(\frac{1}{2}\right)\right] .
$$

Para ilustrar, seguem-se alguns exemplos de cópulas de valores extremos. As cópulas são apresentadas com suas respectivas expressões da função $A(t)$ e as curvas de níveis da densidade da cópula correspondente para diversos valores de $\theta$.

\section{Cópula Gumbel-Hougaard}

$$
C\left(u_{1}, u_{2}\right)=\exp \left\{-\left[\left(-\ln u_{1}\right)^{\theta}+\left(-\ln u_{2}\right)^{\theta}\right]^{\frac{1}{\theta}}\right\}
$$

onde $\theta \in[1, \infty)$. A função de Pickands é expressa por,

$$
A(t)=\left[t^{\theta}+(1-t)^{\theta}\right]^{1 / \theta}
$$


Sua curva de densidade já foi descrita na seção anterior.

\section{Cópula Tawn}

$$
A(t)=1-\beta+(\beta-\alpha) t+\left[(\alpha t)^{\theta}+(\beta(1-t))^{\theta}\right]^{1 / \theta}
$$

Uma extensão Gumbel-Hougaard é a chamada cópula de Tawn que é assimétrica.

A cópula possui função de Pickands $A(t)$ apresentada acima. Da mesma forma temos que $\theta \geq 1$ e $0 \leq \alpha, \beta \leq 1$.
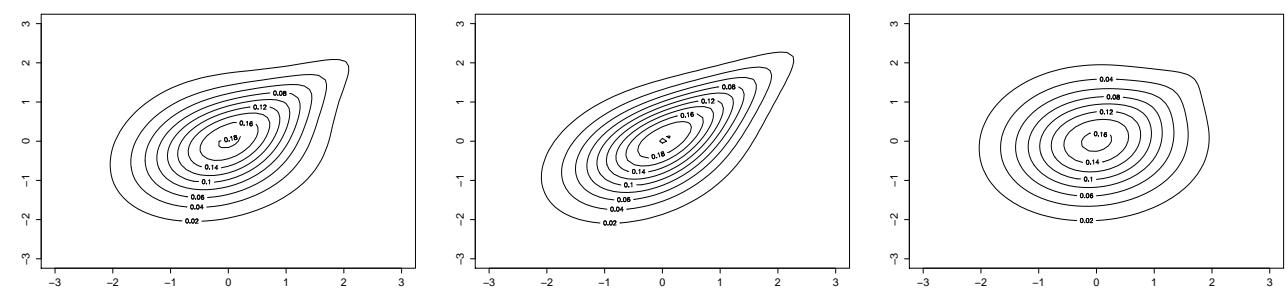

(a) $\theta=7.6, \alpha=0.6, \beta=$ (b) $\theta=7.6, \alpha=0.9, \beta=$ (c) $\theta=10, \alpha=0.2, \beta=$ 0.9

0.6

0.9

Figura 1.13: Tawn $c_{\theta_{i}, \alpha, \beta}\left(F\left(x_{1}\right), F\left(x_{2}\right)\right), i=1,2,3$, marginal normal padrão

\section{Cópula Husler-Reiss}

$$
C\left(u_{1}, u_{2}\right)=\exp \left\{-\tilde{u}_{1} \Phi\left[\frac{1}{\theta}+\frac{1}{2} \theta \log \left(\frac{\tilde{u}_{2}}{\tilde{u}_{1}}\right)\right]-\tilde{u}_{2} \Phi\left[\frac{1}{\theta}+\frac{1}{2} \theta \log \left(\frac{\tilde{u}_{1}}{\tilde{u}_{2}}\right)\right]\right\}
$$

em que $\tilde{u}=-\log (u), \theta \in[0, \infty)$ e $\Phi$ é a função de distribuição acumulada da normal padrão. A função de Pickands desta cópula é dada por,

$$
A(t)=t \Phi\left[\theta^{-1}+\frac{1}{2} \theta \log \left(\frac{t}{1-t}\right)\right]+(1-t) \Phi\left[\theta^{-1}-\frac{1}{2} \theta \log \left(\frac{1-t}{t}\right)\right] .
$$




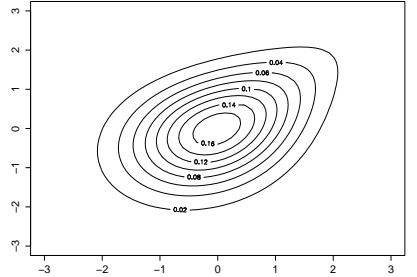

(a) $\theta=1$

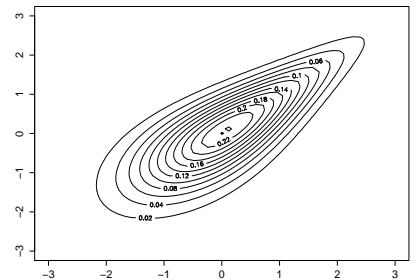

(b) $\theta=2$

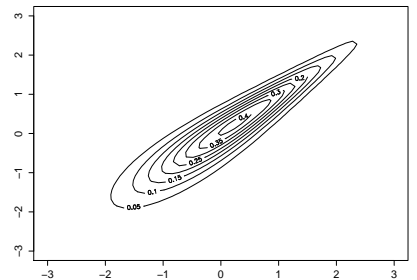

(c) $\theta=4$

Figura 1.14: Husler-Reiss $c_{\theta_{i}}\left(F\left(x_{1}\right), F\left(x_{2}\right)\right), i=1,2,3$, marginal normal padrão

\section{Cópula Galambos}

$$
C\left(u_{1}, u_{2}\right)=u_{1} u_{2} \exp \left\{\left[\tilde{u}_{1}^{-\theta}+{\tilde{u_{2}}}^{-\theta}\right]^{-\frac{1}{\theta}}\right\},
$$

em que $\tilde{u}=-\log (u), \theta \in[0, \infty)$ e possui a seguinte função Pickands,

$$
A(t)=1-\left(t^{-\theta}+(1-t)^{-\theta}\right)^{-1 / \theta} .
$$

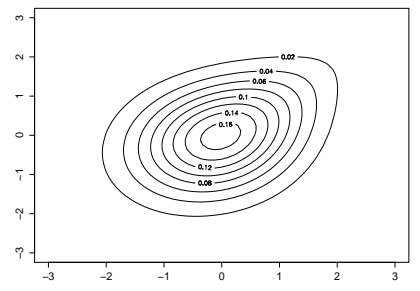

(a) $\theta=0.5$

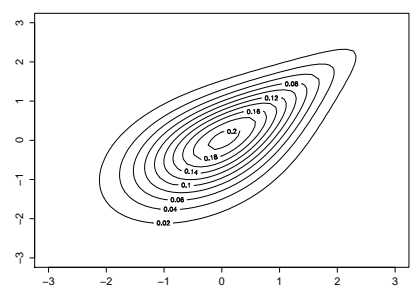

(b) $\theta=1$

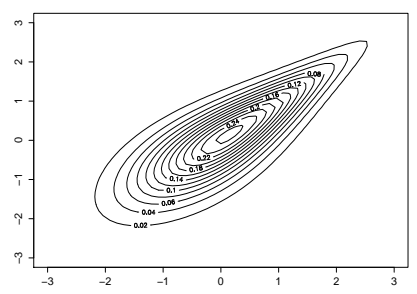

(c) $\theta=1.6$

Figura 1.15: Galambos $c_{\theta_{i}}\left(F\left(x_{1}\right), F\left(x_{2}\right)\right), i=1,2,3$, marginal normal padrão

Na figura abaixo são mostradas as curvas da função de Pickands para cada uma das cópulas de valores extremos apresentadas. 


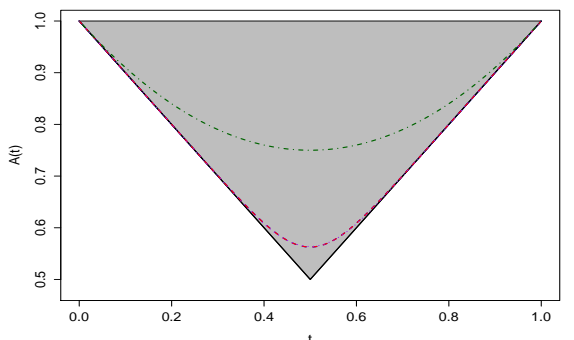

(a) $\tau_{C}=0.83$

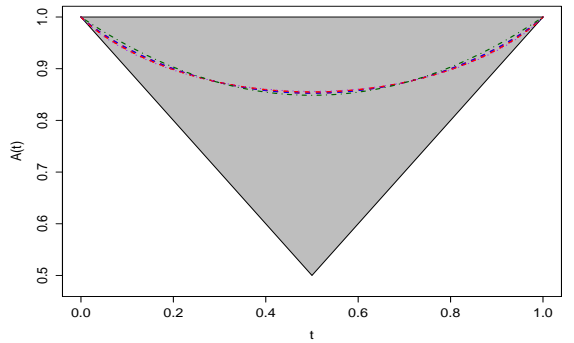

(b) $\tau_{C}=0.23$

Figura 1.16: Galambos (vermelho), Husler-Reiss (lilás), Tawn (verde) e Gumbel (azul) 


\subsection{Misturas de Cópulas}

Uma maneira de criar novas famílias de cópulas é através da realização de misturas finitas. Apesar da gama de classes e famílias de cópulas e suas variedades em termos de estrutura de dependência, a utilização de misturas permite herdar características distintas de várias cópulas em uma mesma cópula. O objetivo da mistura de cópulas é produzir estruturas de dependência mais flexíveis. As combinações, em algumas situações, são vantajosas em especial na área de finanças. Para ilustrar, Arakelian e Karlis (2014) citam o uso de mistura de cópulas no caso em que um par ou mais de ativos possuem estruturas de correlações distintas quando ocorrem más e boas notícias nos mercados.

Nesta seção tratamos brevemente das medidas de dependência da mistura de cópulas. Ilustramos também a estrutura da dependência resultante da combinação de cópulas distintas com medidas de dependência também distintas.

Uma família de cópula gerada a partir de uma mistura finita pode ser expressa da seguinte forma,

$$
C\left(u_{1}, u_{2}\right)=\sum_{i=1}^{k} w_{i} C_{i}\left(u_{1}, u_{2} ; \theta_{i}\right),
$$

em que $\sum_{i=1}^{k} w_{i}=1,0 \leq w_{i} \leq 1$ e $C_{i}$ é uma função cópula, sendo $\theta_{i}$ o vetor de parâmetros de dependência correspondente a $i$-ésima cópula. As $k$ componentes da mistura podem pertencer a famílias diferentes de cópulas. Da mesma forma que foi feita nas seções anteriores, vamos focar nos casos de cópulas bivariadas e com $k=2$. No entanto, os resultados podem ainda ser estendidos para $k>2$. Para tal, trataremos de mistura de cópulas com a seguinte configuração,

$$
C\left(u_{1}, u_{2}\right)=w C_{1}\left(u_{1}, u_{2} ; \theta_{1}\right)+(1-w) C_{2}\left(u_{1}, u_{2} ; \theta_{2}\right)
$$

Podemos perceber que a mistura de cópulas também é uma função cópula, bas- 
tando para isso verificar os quatro pontos da Definição 4.

Alguns pontos que surgem é quanto as medidas de dependência resultante da mistura. No caso do $\tau(C)$ temos que,

$$
\begin{aligned}
\tau(C)=Q(C, C) & =4 \iint_{\boldsymbol{I}^{2}} C\left(u_{1}, u_{2}\right) d C\left(u_{1}, u_{2}\right)-1 \\
& =4 \iint_{\boldsymbol{I}^{2}}\left(w C_{\theta_{1}}+(1-w) C_{\theta_{2}}\right)\left(w d C_{\theta_{1}}+(1-w) d C_{\theta_{2}}\right)-1 \\
& =4 w^{2} \iint_{\boldsymbol{I}^{2}} C_{\theta_{1}} d C_{\theta_{1}}+4(1-w)^{2} \iint_{\boldsymbol{I}^{2}} C_{\theta_{2}} d C_{\theta_{2}} \\
& +4 w(1-w) \iint_{\boldsymbol{I}^{2}} C_{\theta_{1}} d C_{\theta_{2}}+4 w(1-w) \iint_{\boldsymbol{I}^{2}} C_{\theta_{2}} d C_{\theta_{1}}-1
\end{aligned}
$$

Logo temos que,

$$
\tau(C)=w^{2} \tau\left(C_{\theta_{1}}\right)+(1-w)^{2} \tau\left(C_{\theta_{2}}\right)+2 w(1-w) Q\left(C_{\theta_{1}}, C_{\theta_{1}}\right) .
$$

Note que na última passagem em (1.63) usamos o fato de que a função $Q$ é simétrica em seus argumentos. No caso do $\rho_{S}$ temos o seguinte,

$$
\begin{aligned}
\rho_{S}(C)=3 Q(C, \Pi) & =12 \iint_{\boldsymbol{I}^{2}} u_{1} u_{2} d C\left(u_{1}, u_{2}\right)-3 \\
& =12 \iint_{\boldsymbol{I}^{2}} C\left(u_{1}, u_{2}\right) d u_{1} d u_{2}-3 \\
& =12 \iint_{\boldsymbol{I}^{2}}\left[w C_{\theta_{1}}\left(u_{1}, u_{2}\right)+(1-w) C_{\theta_{2}}\left(u_{1}, u_{2}\right)\right] d u_{1} d u_{2}-3 \\
& =12 w \iint_{\boldsymbol{I}^{2}} C_{\theta_{1}}\left(u_{1}, u_{2}\right) d u_{1} d u_{2}+12(1-w) \iint_{\boldsymbol{I}^{2}} C_{\theta_{2}}\left(u_{1}, u_{2}\right) d u_{1} d u_{2}-3 .
\end{aligned}
$$

Assim, diferentemente do $\tau$ de Kendall, expresso por (1.64), o $\rho_{S}$ de Spearman da combinação convexa de cópulas é a soma dos $\rho_{S}$ individuais ponderado por $w$.

$$
\rho_{S}(C)=w \rho_{S}\left(C_{\theta_{1}}\right)+(1-w) \rho_{S}\left(C_{\theta_{2}}\right) .
$$

A mesma propriedade observada para o $\rho_{S}$ também ocorre para a dependência 
caudal. Se a mistura de cópula possui dependência caudal, então temos que,

$$
\begin{aligned}
\lambda_{L}^{\theta} & =\lim _{u \rightarrow 0^{+}} \frac{C(u, u ; \theta)}{u} \\
& =\lim _{u \rightarrow 0^{+}} \frac{w C_{\theta_{1}}(u, u)+(1-w) C_{\theta_{2}}(u, u)}{u} \\
& =w \lambda_{L}^{\theta_{1}}+(1-w) \lambda_{L}^{\theta_{2}} .
\end{aligned}
$$

De forma análoga, ocorre para a cauda superior.

$$
\begin{aligned}
\lambda_{U}^{\theta} & =\lim _{u \rightarrow 1^{-}} \frac{1-2 u+C(u, u ; \theta)}{1-u} \\
& =\lim _{u \rightarrow 1^{-}} \frac{1-2 u+w C_{\theta_{1}}(u, u)+(1-w) C_{\theta_{2}}(u, u)}{1-u} \\
& =\lim _{u \rightarrow 1^{-}} \frac{1-2 u+(1-2 u) w-(1-2 u) w+w C_{\theta_{1}}(u, u)+(1-w) C_{\theta_{2}}(u, u)}{1-u} \\
& =\lim _{u \rightarrow 1^{-}} \frac{(1-2 u) w+(1-2 u)(1-w)+w C_{\theta_{1}}(u, u)+(1-w) C_{\theta_{2}}(u, u)}{1-u} \\
& =w \lambda_{U}^{\theta_{1}}+(1-w) \lambda_{U}^{\theta_{2}} .
\end{aligned}
$$

Para visualizar a variedade de estrutura de dependência gerada a partir de misturas de funções cópulas, seguem os gráficos das curvas de nível de algumas misturas de cópulas. A Figura 1.17 mostra a mistura de cópulas,

$$
C\left(u_{1}, u_{2}\right)=w C_{\text {Gumbel }}\left(u_{1}, u_{2} ; \theta=2\right)+(1-w) C_{\text {Frank }}\left(u_{1}, u_{2} ; \theta=-4.5\right),
$$

para vários valores de $w$. A mistura foi feita com cópulas com sinais distintos de correlações, sendo $\tau\left(C_{\text {Gumbel }}\right)=0.5$ e $\tau\left(C_{\text {Frank }}\right)=-0.42$. Para as marginais foram utilizadas distribuições normais padrão. 


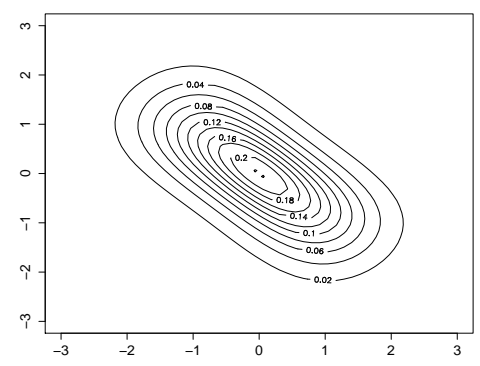

(a) $w=0$

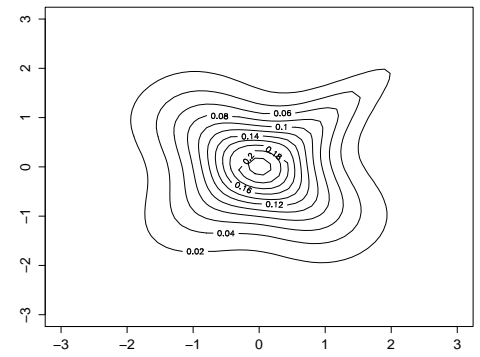

(d) $w=0,4$

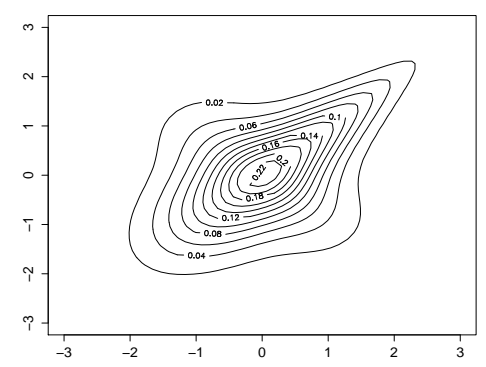

(g) $w=0,75$

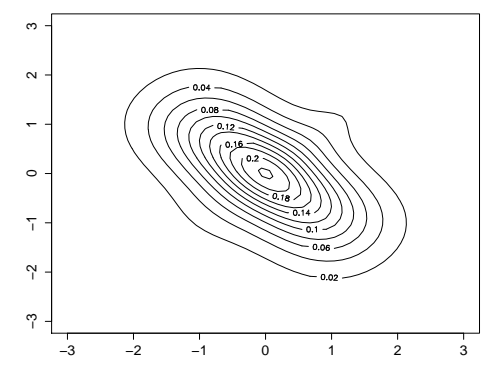

(b) $w=0,1$

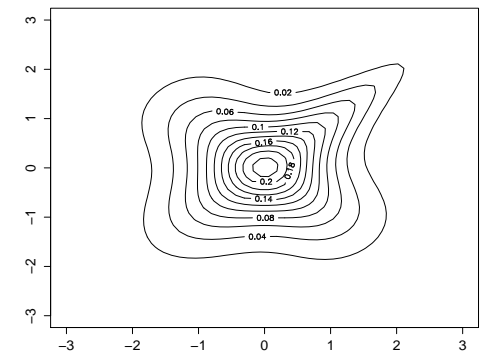

(e) $w=0,5$

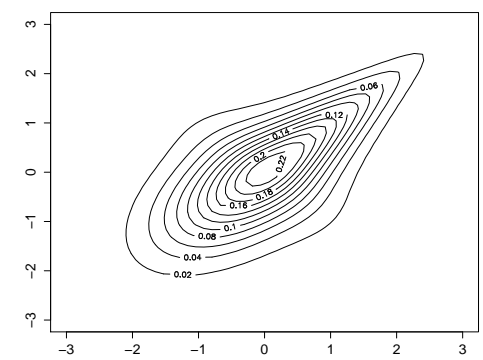

(h) $w=0,9$

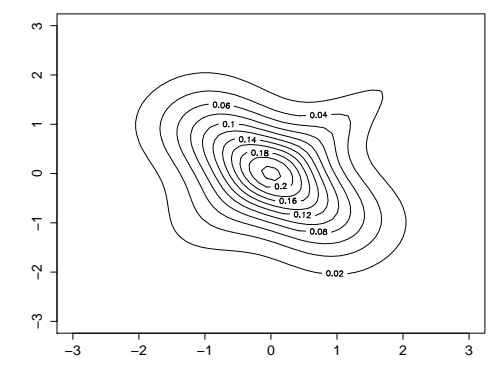

(c) $w=0,25$

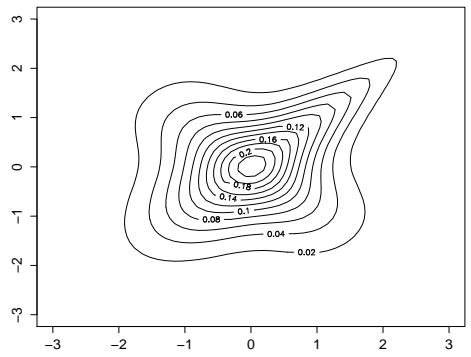

(f) $w=0,6$

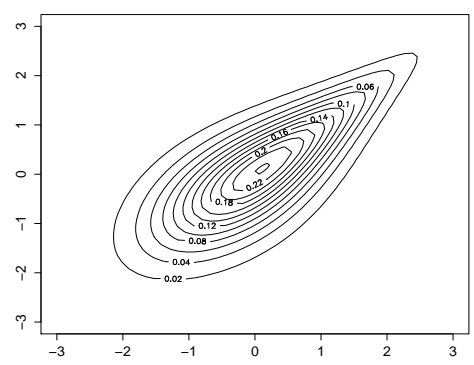

(i) $w=1$

Figura 1.17: Mistura: $w \times \operatorname{Gumbel}(\theta=2)+(1-w) \times \operatorname{Frank}(\theta=-4,5)$

Adicionalmente, podemos ilustrar outro caso de mistura entre uma cópula Joe e Clayton. A mistura é dada por,

$$
C\left(u_{1}, u_{2}\right)=w C_{J o e}\left(u_{1}, u_{2}\right)+(1-w) C_{\text {Clayton }}\left(u_{1}, u_{2}\right) .
$$

O gráfico dessa mistura é apresentado abaixo. 


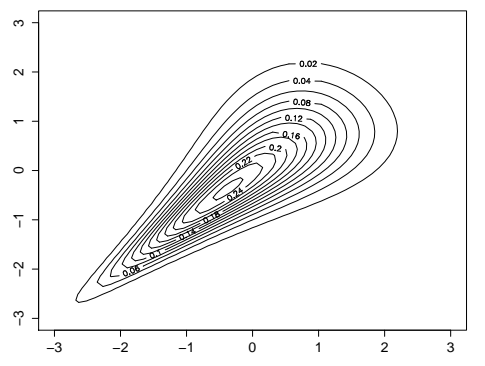

(a) $w=0$

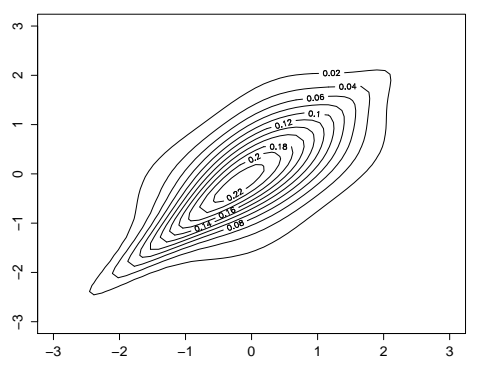

(d) $w=0,4$

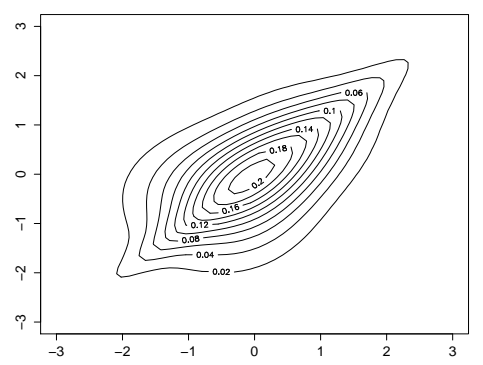

(g) $w=0,75$

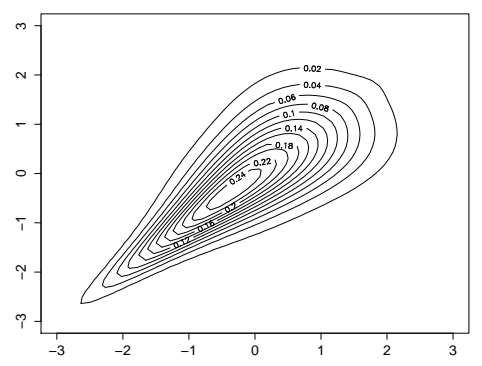

(b) $w=0,1$

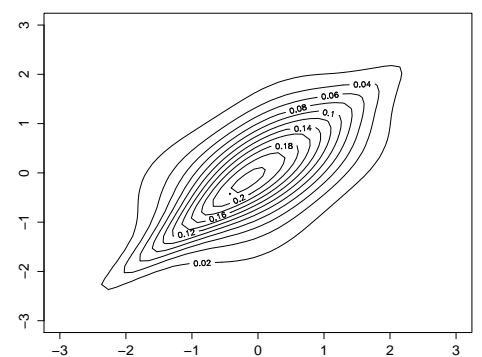

(e) $w=0,5$

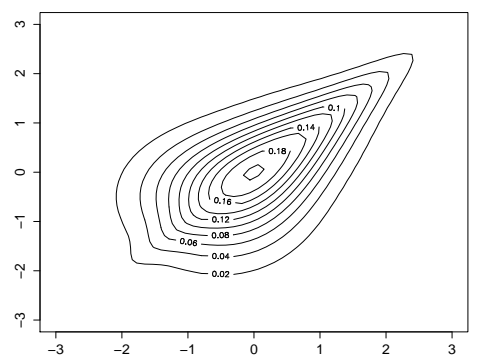

(h) $w=0,9$

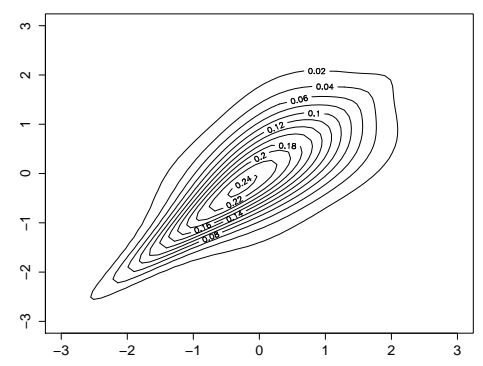

(c) $w=0,25$

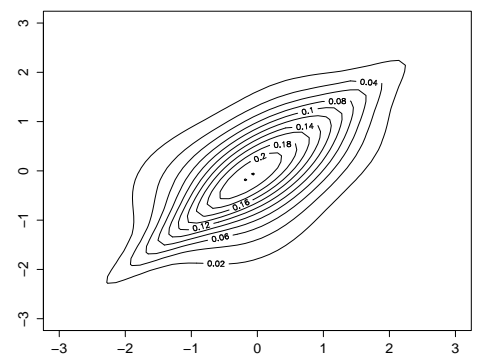

(f) $w=0,6$

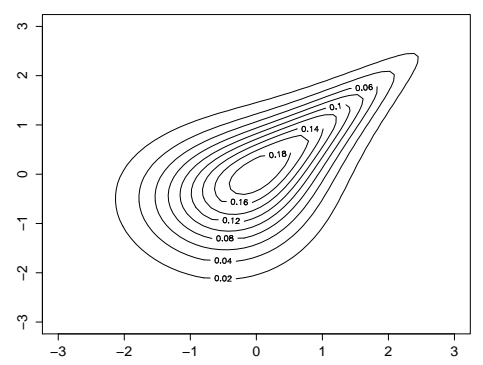

(i) $w=1$

Figura 1.18: Mistura: $w \times \operatorname{Joe}(\theta=2)+(1-w) \times \operatorname{Clayton}(\theta=2,3)$ 


\section{Capítulo 2}

\section{R-Vines Cópulas}

O emprego de cópulas ocorre em vários ramos de estudos, como citado anteriormente. Entretanto, muitas famílias de cópulas se restringem a uma estrutura bidimensional. Na literatura, há algumas cópulas com $n>2$ dimensões. As classes de cópulas $n$-variadas, porém, são restritas, sendo boa parte com estruturas elípticas ou com cópulas arquimedianas. Em geral, as cópulas multivariadas padrão são descritas por um único parâmetro. Esta característica faz com que as estruturas de dependência em altas dimensões sejam inflexíveis.

Neste capítulo, vamos abordar uma alternativa para a construção de cópulas multivariadas para $n \geq 3$. Este tipo de construção teve origem no trabalho de Joe (1996), em que a distribuição multivariada é dada a partir das marginais e de $n(n-1) / 2$ parâmetros de dependência um para cada par de marginais. A elaboração destas cópulas é feita apenas por pares de cópulas, usualmente bivariadas, daí ser conhecido na literatura como construção de cópulas por pares, também denominado $\mathrm{PCC}^{1}$. A decomposição entre pares de variáveis não é única e sua forma de construção traz a desvantagem quanto ao aumento no número de parâmetros da distribuição $n$-variada.

Para organizar a decomposição entre os pares de variáveis, Bedford e Cooke (2002) introduziram uma estrutura gráfica chamada de Regular (R-) Vines. Em

\footnotetext{
${ }^{1}$ Pair Copulas Constructions
} 
especial, há duas classes de R-Vines cópulas, a saber: Canonical (C-) Vines e a Drawable (D-) Vines. Elas se diferenciam, em suma, na configuração da estrutura de dependência entre os pares de variáveis. Estas duas classes são importantes devido a suas aplicações em várias áreas de pesquisa.

Iniciamos este capítulo com uma breve descrição das cópulas formadas por pares, conforme Joe (1996). Em seguida, apresentamos a construção das R-Vines Cópulas, exibindo os esquemas de construção particulares para as D-Vines e CVines cópulas.

\subsection{Distribuição Multivariada por Pares de Cópu- las}

Dado um vetor aleatório $\left(X_{1}, \ldots, X_{n}\right)$ com distribuição acumulada $F_{1: n}$ e distribuições marginais acumuladas $F_{i}, i=1, \ldots, n$ e $C$ a função cópula associada a $\left(F_{1}\left(X_{1}\right), \ldots, F_{n}\left(X_{n}\right)\right)$, o ponto de partida para escrever a distribuição $n$-variada a partir de cópulas bivariadas, mais uma vez, é o Teorema de Sklar. Desta forma, a distribuição $F_{1: n}$ pode ser expressa através de uma cópula $C$ por,

$$
\begin{aligned}
F_{1: m}\left(x_{1}, x_{2}, \ldots, x_{n}\right) & =C\left(F_{1}\left(x_{1}\right), F_{2}\left(x_{2}\right), \ldots, F_{n}\left(x_{n}\right) ; \boldsymbol{\theta}\right) \\
& =C\left(u_{1}, u_{2}, \ldots, u_{n} ; \boldsymbol{\theta}\right) .
\end{aligned}
$$

em que novamente $u_{i}=F_{i}\left(x_{i}\right)$ são os valores das distribuições unidimensionais acumuladas da $i$-ésima componente. Aqui, denotaremos a função acumulada condicional e a densidade condicional por $F(\cdot \mid \cdot)$ e $f(\cdot \mid \cdot)$, respectivamente. Utilizaremos também o subscrito $1: n$ para representar os índices $\{1,2,3, \ldots, n-1, n\}$ na distribuição $F_{1: n}=F_{123 \ldots n}$ com suas respectivas marginais $F_{1}, F_{2}, \cdots, F_{n}$.

O parâmetro de dependência $\theta_{j k}$ indica a relação da variável $j$ com a variável $k$ e $\boldsymbol{\theta}$ é o vetor dos parâmetros utilizados na construção da cópula. Primeiramente mostramos como escrever $F_{1: 3}$ em termos de cópulas bivariadas, dada pela 
expressão,

$$
F_{123}\left(x_{1}, x_{2}, x_{3} ; \boldsymbol{\theta}\right)=\int_{-\infty}^{x_{1}} C_{23 \mid 1}\left(F_{2 \mid 1}\left(x_{2} \mid z_{1} ; \theta_{21}\right), F_{3 \mid 1}\left(x_{3} \mid z_{1} ; \theta_{31}\right) ; \theta_{23}\right) d F_{1}\left(z_{1}\right) .
$$

A partir de cópulas bidimensionais, iniciamos a montagem da densidade $f_{1: 3}\left(x_{1}, x_{2}, x_{3}\right)$, a partir da seguinte decomposição,

$$
f_{1: 3}\left(x_{1}, x_{2}, x_{3}\right)=f_{1}\left(x_{1}\right) f_{2 \mid 1}\left(x_{2} \mid x_{1}\right) f_{3 \mid 21}\left(x_{3} \mid x_{2}, x_{1}\right) .
$$

A estratégia é re-escrever as densidades condicionais em termos das densidades cópulas. O segundo termo de (2.3) é expresso por,

$$
\begin{aligned}
f_{2 \mid 1}\left(x_{2} \mid x_{1}\right) & =\frac{f_{12}\left(x_{1}, x_{2}\right)}{f_{1}\left(x_{1}\right)} \\
& =c_{12}\left(F_{1}\left(x_{1}\right), F_{2}\left(x_{2}\right)\right) f_{2}\left(x_{2}\right),
\end{aligned}
$$

onde o último passo é obtido substituindo a função $f_{12}$ pela equação (1.17), dada no capítulo anterior. O terceiro e último termo de (2.3) é escrito em termos da cópula densidade $c_{23 \mid 1}$, que está associada a $F_{23 \mid 1}\left(x_{2}, x_{3} \mid x_{1}\right)$, e também em termos de $c_{31}$. Temos portanto que,

$$
c_{23 \mid 1}\left(F_{2 \mid 1}\left(x_{2} \mid x_{1}\right), F_{3 \mid 1}\left(x_{3} \mid x_{1}\right) ; x_{1}\right)=\frac{f_{23 \mid 1}\left(x_{2}, x_{3} \mid x_{1}\right)}{f_{2 \mid 1}\left(x_{2} \mid x_{1}\right) f_{3 \mid 1}\left(x_{3} \mid x_{1}\right)} .
$$

Desta forma, escrevemos $f_{3 \mid 21}$ utilizando a equação (2.5) da seguinte forma,

$$
\begin{aligned}
f_{3 \mid 12}\left(x_{3} \mid x_{2}, x_{1}\right) & =\frac{f_{32 \mid 1}\left(x_{3}, x_{2} \mid x_{1}\right)}{f_{2 \mid 1}\left(x_{2} \mid x_{1}\right)} \\
& =\frac{c_{23 \mid 1}\left(F\left(x_{3} \mid x_{1}\right), F_{2 \mid 1}\left(x_{2} \mid x_{1}\right) ; x_{1}\right) f_{3 \mid 1}\left(x_{3} \mid x_{1}\right) f_{2 \mid 1}\left(x_{2} \mid x_{1}\right)}{f_{2 \mid 1}\left(x_{2} \mid x_{1}\right)} \\
& =c_{32 \mid 1}\left(F_{3 \mid 1}\left(x_{3} \mid x_{1}\right), F_{2 \mid 1}\left(x_{2} \mid x_{1}\right) ; x_{1}\right) f_{3 \mid 1}\left(x_{3} \mid x_{1}\right) \\
& =c_{32 \mid 1}\left(F_{3 \mid 1}\left(x_{3} \mid x_{1}\right), F_{2 \mid 1}\left(x_{2} \mid x_{1}\right) ; x_{1}\right) c_{31}\left(F_{3}\left(x_{3}\right), F_{1}\left(x_{1}\right)\right) f_{3}\left(x_{3}\right) .
\end{aligned}
$$


Finalmente, substituindo (2.6) e (2.4) em (2.3), temos a função densidade multivariada,

$$
\begin{aligned}
f_{1: 3}\left(x_{1}, x_{2}, x_{3}\right) & =f_{1}\left(x_{1}\right) \underbrace{c_{12}\left(F_{1}\left(x_{1}\right), F_{2}\left(x_{2}\right)\right) f_{2}\left(x_{2}\right)}_{f_{2 \mid 1}\left(x_{2} \mid x_{1}\right)} \\
& \times \underbrace{c_{32 \mid 1}\left(F_{3 \mid 1}\left(x_{3} \mid x_{1}\right), F_{2 \mid 1}\left(x_{2} \mid x_{1}\right) ; x_{1}\right) c_{31}\left(F_{3}\left(x_{3}\right), F_{1}\left(x_{1}\right)\right) f_{3}\left(x_{3}\right)}_{f_{3 \mid 21}\left(x_{3} \mid x_{2}, x_{1}\right)} \\
& =\underbrace{f_{1}\left(x_{1}\right) f_{2}\left(x_{2}\right) f_{3}\left(x_{3}\right)}_{\text {Cópulas Não Condicionais }} \underbrace{c_{12}\left(F_{1}\left(x_{1}\right), F_{2}\left(x_{2}\right)\right) c_{31}\left(F_{3}\left(x_{3}\right), F_{1}\left(x_{1}\right)\right)}_{\text {Marginais }} \\
& \times \underbrace{c_{32 \mid 1}\left(F_{3 \mid 1}\left(x_{3} \mid x_{1}\right), F_{2 \mid 1}\left(x_{2} \mid x_{1}\right) ; x_{1}\right)}_{\text {Cópulas Condicionais }} .
\end{aligned}
$$

Para obter a distribuição acumulada $F_{1: 3}$ integramos (2.7) com relação a $x_{1}, x_{2}$ e $x_{3}$.

$$
\begin{aligned}
& F_{1: 3}\left(x_{1}, x_{2}, x_{3}\right) \\
& =\int_{-\infty}^{x_{1}} \int_{-\infty}^{x_{2}} \int_{-\infty}^{x_{3}} f_{1: 3}\left(z_{1}, z_{2}, z_{3}\right) d F_{1}\left(z_{1}\right) d F_{2}\left(z_{2}\right) d F_{3}\left(z_{3}\right) \\
& =\int_{-\infty}^{x_{1}} \int_{-\infty}^{x_{2}} \int_{-\infty}^{x_{3}} f_{1}\left(z_{1}\right) f_{2}\left(z_{2}\right) f_{3}\left(z_{3}\right) c_{12}\left(F_{1}\left(z_{1}\right), F_{2}\left(z_{2}\right)\right) c_{31}\left(F_{3}\left(z_{3}\right), F_{1}\left(z_{1}\right)\right) \\
& \times c_{32 \mid 1}\left(F_{3 \mid 1}\left(z_{3} \mid z_{1}\right), F_{2 \mid 1}\left(z_{2} \mid z_{1}\right) ; z_{1}\right) d F_{3}\left(z_{3}\right) d z_{3} d z_{2} d z_{1} \\
& =\int_{-\infty}^{x_{1}} \int_{-\infty}^{x_{2}} \int_{-\infty}^{x_{3}} f_{1}\left(z_{1}\right) f_{2 \mid 1}\left(z_{2} \mid z_{1}\right) f_{3 \mid 1}\left(z_{3} \mid z_{1}\right) c_{23 \mid 1}\left(F_{2 \mid 1}\left(z_{2} \mid z_{1}\right), F_{3 \mid 1}\left(z_{3} \mid z_{1}\right) ; z_{1}\right) d z_{3} d z_{2} d z_{1} \\
& =\int_{-\infty}^{x_{1}} \int_{-\infty}^{x_{2}} \int_{-\infty}^{x_{3}} \frac{\partial^{2} C_{23 \mid 1}\left(F_{2 \mid 1}\left(z_{2} \mid z_{1}\right), F_{3 \mid 1}\left(z_{3} \mid z_{1}\right) ; z_{1}\right)}{\partial z_{3} \partial z_{2}} d z_{3} d z_{2} d F_{1}\left(z_{1}\right) \\
& =\int_{-\infty}^{x_{1}} C_{23 \mid 1}\left(F_{2 \mid 1}\left(z_{2} \mid z_{1}\right), F_{3 \mid 1}\left(z_{3} \mid z_{1}\right) ; z_{1}\right) d F_{1}\left(z_{1}\right) .
\end{aligned}
$$

Na passagem (2.8a) utilizamos a equação (2.4) e na (2.8b) a equação (1.17). Deste modo, obtemos a função dada por (2.2).

Repare que tanto a função de distribuição acumulada (2.8) quanto a densidade (2.7) depende da variável $x_{1}$. Isto ocorre não apenas de forma indireta, por meio dos argumentos $F_{2 \mid 1}\left(x_{2} \mid x_{1}\right)$ e $F_{3 \mid 1}\left(x_{3} \mid x_{1}\right)$, mas também de forma direta por 
$x_{1}$. A expressão (2.7) é denominada de PCC Completa ${ }^{2}$. Basicamente existem duas razão para não utilizar a PCC completa. A primeira é do ponto de vista prático o segundo é do ponto de vista inferencial. A estimação com PCC usual assume independência da cópula condicional com a variável condicionada a não ser pelas distribuições condicionais. Assumindo tal suposição a construção da distribuição multivariada é denotada por PCC Simplificada ${ }^{3}$ (Haff et al., 2010). No caso tridimensional a PCC Simplificada fica expressa da seguinte forma,

$$
\begin{aligned}
f_{1: 3}\left(x_{1}, x_{2}, x_{3}\right) & =f_{1}\left(x_{1}\right) f_{2}\left(x_{2}\right) f_{3}\left(x_{3}\right) c_{12}\left(F_{1}\left(x_{1}\right), F_{2}\left(x_{2}\right)\right) c_{31}\left(F_{3}\left(x_{3}\right), F_{1}\left(x_{1}\right)\right) \\
& \times c_{32 \mid 1}\left(F_{3 \mid 1}\left(x_{3} \mid x_{1}\right), F_{2 \mid 1}\left(x_{2} \mid x_{1}\right)\right) .
\end{aligned}
$$

Haff et al. (2010) destaca que a suposição PCC Simplificada não é severa para fins de estimação dos parâmetros. Vamos considerar aqui apenas a decomposição de pares de cópulas em que a cópula densidade condicional que não dependa diretamente da variável condicionada.

O caso $n$-dimensional de (2.9), é obtido seguindo a mesma construção empregada no caso $n=3$. Para isso considere a notação $\boldsymbol{x}_{i: j}=\left(x_{i}, x_{i+1}, \ldots, x_{j}\right)$. A decomposição $n$-dimensional, utilizada em (2.3), é expressa por,

$$
f_{1: n}\left(x_{1}, x_{2}, \ldots, x_{n}\right)=\prod_{i=2}^{n} f_{i \mid 1:(i-1)}\left(x_{i} \mid \boldsymbol{x}_{1:(i-1)}\right) f_{1:(i-1)}\left(\boldsymbol{x}_{1:(i-1)}\right)
$$

Cada termo do produtório (2.10) pode ser escrito por,

$$
\begin{aligned}
f_{i \mid 1:(i-1)}\left(x_{i} \mid \boldsymbol{x}_{1:(i-1)}\right) & =c_{1 i \mid 2:(i-1)}\left(F_{1 \mid 2:(i-1)}\left(x_{1} \mid \boldsymbol{x}_{2:(i-1)}\right), F_{i \mid 2:(i-1)}\left(x_{i} \mid \boldsymbol{x}_{2:(i-1)}\right)\right) \\
& \times f_{i \mid 2:(i-1)}\left(x_{i} \mid \boldsymbol{x}_{2:(i-1)}\right) .
\end{aligned}
$$

Novamente, considerando a distribuição de $F_{1 \mid 2:(i-1)}$ e $F_{i \mid 2:(i-1)}$ não depen-

\footnotetext{
${ }^{2}$ Full PCC

${ }^{3}$ Simplified PCC
} 
dente de $\boldsymbol{x}_{2:(i-1)}$ e aplicando recursivamente (2.11) temos,

$$
\begin{aligned}
& f_{i \mid 1:(i-1)}\left(x_{i} \mid x_{1}, \ldots, x_{j-1}\right) \\
& =\prod_{k=1}^{i-2} c_{k i \mid(k+1):(i-1)}\left(F_{k \mid(k+1):(i-1)}\left(x_{k} \mid \boldsymbol{x}_{(k+1):(i-1)}\right), F_{i \mid(j+1):(i-1)}\left(x_{i} \mid \boldsymbol{x}_{(j+1):(i-1)}\right)\right) \\
& \times c_{i-1, i}\left(F_{i-1}\left(x_{i-1}\right), F_{i}\left(x_{i}\right)\right) f_{i}\left(x_{i}\right) .
\end{aligned}
$$

Substituindo a equação (2.12) em (2.10) obtemos a generalização de (2.7).

$$
\begin{aligned}
& f\left(x_{1}, x_{2}, \ldots, x_{n}\right) \\
& =\prod_{j=1}^{n} f_{j}\left(x_{j}\right) \prod_{i=1}^{n} \prod_{k=1}^{i-1} c_{k i \mid(k+1):(i-1)}\left(F_{k \mid(k+1):(i-1)}\left(x_{k} \mid \boldsymbol{x}_{(k+1):(i-1)}\right), F_{i \mid(k+1):(i-1)}\left(x_{i} \mid \boldsymbol{x}_{(k+1):(i-1)}\right)\right) .
\end{aligned}
$$

A sequência dos pares de cópula apresentada em (2.13) não é única, logo outras configurações para a expressão acima também são possíveis.

A generalização da equação (2.2) para o caso $n$-dimensional é feita integrando (2.13) com relação a $x_{1}, \ldots, x_{n}$.

$$
\begin{aligned}
& F_{1: n}\left(x_{1}, \cdots, x_{n}\right) \\
& =\int_{-\infty}^{x_{2}} \cdots \int_{-\infty}^{x_{n-1}} C_{1 n}\left(F_{1 \mid 2 \cdots n-1}\left(x_{1} \mid z_{2}, \cdots, z_{n-1} ; \theta_{12}, \cdots, \theta_{n-2, n-1}\right)\right. \\
& F_{n \mid 2 \cdots n-1}\left(x_{n} \mid z_{2}, \cdots, z_{n-1} ; \theta_{23}, \cdots, \theta_{n-1, n}\right) d F_{2 \cdots n-1}\left(z_{2}, \cdots, z_{n-1} ; \theta_{23}, \cdots, \theta_{n-2, n-1}\right) .
\end{aligned}
$$

Para descrever o caso geral, vamos introduzir a seguinte notação $\boldsymbol{\nu}=\left\{k, j_{1}, \ldots, j_{n-2}\right\}$ em que $k<j$ onde o subscrito $-k$ indica $\boldsymbol{\nu}_{-k}=\left\{j_{1}, \ldots, j_{n-2}\right\}$, isto é, todos os elementos de $\boldsymbol{\nu}$ exceto o de índice $k$. A distribuição acumulada condicional para qualquer $n$ é dada por,

$$
F_{j \mid \boldsymbol{\nu}}\left(x_{j} \mid \boldsymbol{x}_{\boldsymbol{\nu}}\right)=D_{1} C_{k j \mid \boldsymbol{\nu}_{-k}}\left(F_{k \mid \boldsymbol{\nu}_{-k}}\left(x_{k} \mid \boldsymbol{x}_{\boldsymbol{\nu}_{-k}}\right), F_{j \mid \boldsymbol{\nu}_{-k}}\left(x_{j} \mid \boldsymbol{x}_{\boldsymbol{\nu}_{-k}}\right)\right) .
$$

Perceba que a distribuição acumulada condicional (2.15) tem a vantagem de ser 
obtida recursivamente. Mais detalhes podem ser vistos em Joe (1996).

\subsection{Vine Cópulas}

A combinação dos pares de cópulas para a construção da distribuição $n$ variada descrita na seção anterior não é única. Em função disto, Bedford e Cooke (2002) desenvolveram um diagrama com uma sequência de árvores para organizar o conjunto pares, no qual chamaram de Regular Vines ou abreviadamente R-Vines. As árvores são compostas por nós (nodes) e por laços (edges). A montagem do diagrama de uma R-Vine $n$-dimensional é composta por uma sequência de $n-1$ árvores, sendo,

\section{Árvore $1 A_{1}$}

$n$ nós: $X_{1}, X_{2}, \ldots, X_{n}$.

$n-1$ laços: cada laço conecta um par das variáveis aleatórias $X_{1}, X_{2}, \ldots, X_{n}$ representado pela densidade cópula.

Árvore $\mathbf{j} A_{j}$ para $j=2,3, \ldots, n-1$

$1 n+1-j$ nós: cada nó é representado pelos laços da árvore anterior.

$2 n-j$ laços: cada laço conecta um nó representado pela densidade cópula condicional.

A construção do diagrama segue a condição de proximidade, no qual se dois nós são ligados por um laço na árvore $j$, então eles irão compor um nó na árvore $j+1$. Os laços definem os pares das densidades das cópulas que serão inseridas na fórmula (2.13). Na construção do diagrama das R-Vines, duas variantes foram identificadas por Bedford e Cooke (2002), as C-Vines e as D-Vines. Suas diferenças residem nos seguintes pontos:

D-Vine: Cada nó tem no máximo 2 laços para todas árvores $A_{j}$. 
C-Vine: Cada árvore $A_{j}$ tem um único nó com $n-j$ laços. O primeiro nó é chamado de raiz.

Um exemplo de diagrama $\mathrm{D}$ e $\mathrm{C}$ Vines para $n=5$ encontram-se abaixo.

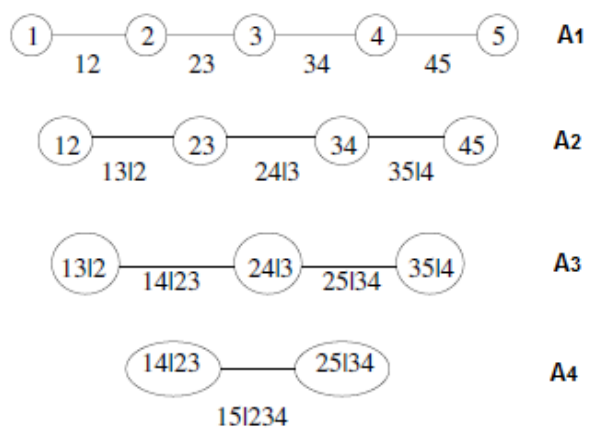

(a) D-Vine:

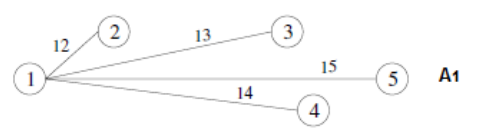

(12) $\frac{2311}{2411} \quad 2511$ (1) 15 A2

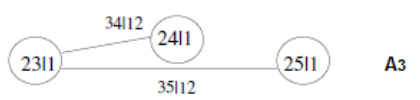

$34112 \underset{451123}{3} \quad \mathrm{~A} 4$

(b) C-Vine:

Figura 2.1: Diagrama R-Vine para Drawable (D) e Canonical (C) Vines cópulas com $n=5$

A modelagem de uma distribuição multivariada se torna completa quando se determina a distribuição das marginais com seus parâmetros e a família de funções cópulas com seus respectivos valores de $\boldsymbol{\theta}$. De forma geral, a modelagem de uma distribuição multivariada pode ser descrita por três componentes.

\section{Modelo Multivariado = Estrutura R-Vine + Família Cópula + Marginais.}

A estrutura indica a forma de construção entre as variáveis; a família descreve a estrutura de dependência entre as variáveis definidas pela arquitetura do diagrama R-Vine e, por último, a distribuição das marginais e seus parâmetros. Para melhor ilustrar considere o exemplo seguinte.

Exemplo 3 Considere o diagrama da D-Vine com $n=3$ abaixo com as variáveis aleatórias $X_{1}, X_{2}$ e $X_{3}$ e suas respectivas marginais $F_{1}, F_{2}, F_{3}$. 


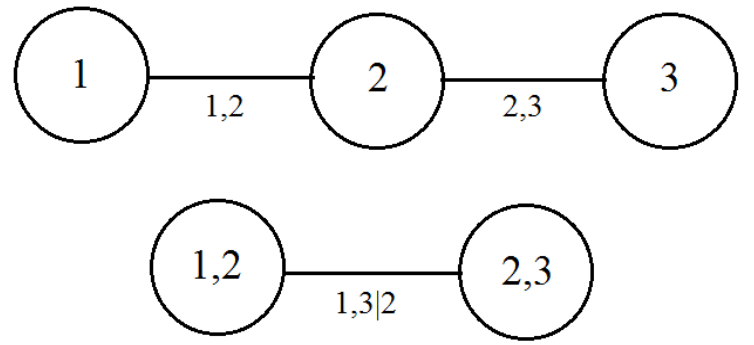

Figura 2.2: Diagrama D-Vine $m=3$

Suponha que as associações entre as variáveis sejam descritas pelas cópulas Gaussiana $\left(C_{12}^{G}\right)$, Frank $\left(C_{23}^{F}\right)$ e Clayton $\left(C_{13 \mid 2}^{C}\right)$, como mostra o diagrama na Figura 2.3. Considere ainda que as todas as marginais são normais padrão, isto é, $X_{i} \sim$ $N(0,1)$, com função distribuição acumulada $F_{i}\left(x_{i}\right)=\Phi_{i}\left(x_{i}\right)$ para $i=1,2,3$.

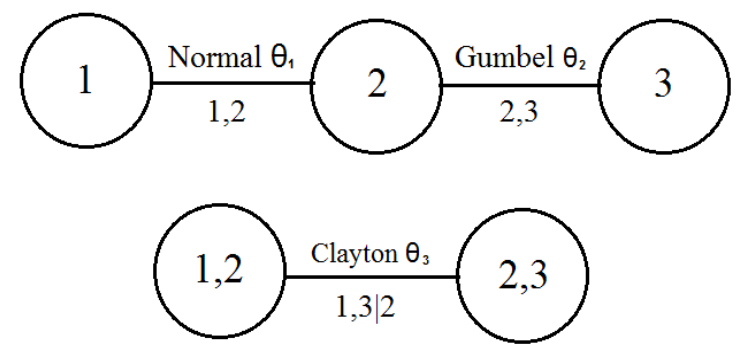

Figura 2.3: Exemplo diagrama D-Vine $m=3$

Através do Diagrama (2.3) e seguindo a equação (2.13), a modelagem da distribuição multivariada de $\left(X_{1}, X_{2}, X_{3}\right)$ com o conjunto de parâmetros $\boldsymbol{\theta}=\left(\theta_{1}, \theta_{2}, \theta_{3}\right)$ fica,

$$
\begin{aligned}
f\left(x_{1}, x_{2}, x_{3}\right)= & f_{1}\left(x_{1}\right) f_{2}\left(x_{2}\right) f 3\left(x_{3}\right) c_{12}^{G}\left(\Phi_{1}\left(x_{1}\right), \Phi_{2}\left(x_{2}\right) ; \theta_{1}\right) \\
& \times c_{23}^{F}\left(\Phi_{2}\left(x_{2}\right), \Phi_{3}\left(x_{3}\right) ; \theta_{2}\right) c_{13 \mid 2}^{C}\left(\Phi_{1 \mid 2}\left(x_{1} \mid x_{2}\right), \Phi_{3 \mid 2}\left(x_{3} \mid x_{2}\right) ; \theta_{3}\right) .
\end{aligned}
$$

Uma vantagem da utilização das R-Vines é a possibilidade de realizar amostragens oriundas de distribuições multivariadas empregando as técnicas de simulação descritas nas seções anteriores com uso de cópulas. Outro ponto importante 
é quando as variáveis aleatórias $\boldsymbol{X}$ são ordenadas no tempo i.e. $X_{t}$ é observado antes de $X_{t+1}$, então as Vines cópulas, em especial a D-Vine, permitem uma forma natural de avaliar uma dependência serial. No Capítulo 4 será apresentado brevemente como as R-Vines cópulas podem ser utilizadas para construir um GARCH multivariado. 


\section{Capítulo 3}

\section{Distribuição de Funções de Variáveis}

\section{Aleatórias via Funções Cópulas}

Dadas as variáveis aleatórias $X_{1}, \ldots, X_{n}$, definidas sob o mesmo espaço de probabilidade $(\Omega, \mathcal{F}, \boldsymbol{P})$, diversas funções dessas variáveis, $g_{n}\left(X_{1}, \ldots, X_{n}\right)$ são de grande interesse na teoria estatística e em outras áreas da estatística aplicada. Na inferência estatística, por exemplo, variáveis aleatórias com distribuição QuiQuadrado, Gama, Estáveis, vem da soma $X_{1}+\cdots+X_{n}$ das $X_{i}$ 's independentes. Já as variáveis aleatórias com distribuição t-Student, F de Snedecor, Slash são provenientes de quocientes de variáveis aleatórias independentes.

Em diversas aplicações nas áreas de finanças, atuária, hidrologia, análise de sobrevivência e entre outras, as funções do tipo $\sum_{i=1}^{n} X_{i}, \sum_{i=1}^{n} \pi_{i} X_{i}, \prod_{i=1}^{n} X_{i}$, $X_{1} /\left(\sum_{i=1}^{n} X_{1}\right)$ são de fundamental importância quando as $X_{i}$ 's não são necessariamente independentes. Nesse contexto, o presente capítulo trata da distribuição dessas funções considerando uma estrutura de dependência é induzida por funções cópulas.

Na seção 3.1 exploramos a distribuição da soma e da combinação convexa. Na seção 3.2 apresentamos a distribuição do produto e do quociente. 


\subsection{Convolução via Cópulas}

Preliminarmente vamos introduzir nesta seção a distribuição da soma de duas variáveis aleatórias contínuas $W_{2}=X_{1}+X_{2}$, onde a estrutura de dependência entre $X_{1}$ e $X_{2}$ é dada por uma cópula $C$. Os resultados para este caso são tratados em Cherubini et al. (2011).

Antes de enunciar a formulação da Proposição 2, sobre a distribuição da soma de duas variáveis aleatórias com dependência dada por uma função cópula, enunciamos os dois Lemas seguintes, úteis na demonstração dessa Proposição.

Lema 2 Seja $C\left(u_{1}, u_{2}\right)$ uma cópula, para qualquer $u_{2} \in \boldsymbol{I}$, a derivada parcial $\partial C\left(u_{1}, u_{2}\right) / \partial u_{1}$ existe para quase todo $u_{1} \mathrm{e}$

$$
0 \leq \frac{\partial}{\partial u_{1}} C\left(u_{1}, u_{2}\right) \leq 1
$$

O mesmo ocorre para $u_{2}$, i.e.

$$
0 \leq \frac{\partial}{\partial u_{2}} C\left(u_{1}, u_{2}\right) \leq 1
$$

Adicionalmente, as funções

$$
u_{1} \mapsto \partial C\left(u_{1}, u_{2}\right) / \partial u_{2}, u_{2} \mapsto \partial C\left(u_{1}, u_{2}\right) / \partial u_{1}
$$

são definidas e não decrescentes para quase todo $\boldsymbol{I}$.

O segundo Lema importante consta-se abaixo.

Lema 3 Seja $C\left(u_{1}, u_{2}\right)$ uma função cópula e $F_{X}$ e $F_{Y}$ duas distribuições acumuladas contínuas, então $\forall t \in \mathbb{R}, D_{1} C\left(u_{1}, F_{Y}\left(t-F_{X}^{-1}\left(u_{1}\right)\right)\right)$ está bem definida quase certamente para $u_{1} \in \boldsymbol{I}$.

Com base neste dois Lemas estamos aptos a provar a proposição seguinte.

Proposição 2 (Cherubini et al, 2011) Sejam $X_{1}$ e $X_{2}$ duas variáveis aleatórias 
com mesmo espaço de probabilidade $(\Omega, \mathcal{F}, \boldsymbol{P})$. Sendo $C\left(u_{1}, u_{2}\right)$ a cópula associada as variáveis $X_{1}$ e $X_{2}$ e marginais contínuas $F_{X_{1}}$ e $F_{X_{2}}$, então $\forall t \in \mathbb{R}$ quase certamente temos que a distribuição da soma $X_{1}+X_{2}$ é dada por,

$$
F_{X_{1}+X_{2}}(t)=\int_{0}^{1} D_{1} C\left(u_{1}, F_{X_{2}}\left(t-F_{X_{1}}^{-1}\left(u_{1}\right)\right)\right) d u_{1}
$$

ou de forma equivalente,

$$
F_{X_{1}+X_{2}}(t)=\int_{0}^{1} D_{2} C\left(F_{X_{1}}\left(t-F_{X_{2}}^{-1}\left(u_{2}\right)\right), u_{2}\right) d u_{2}
$$

Prova. Para verificar a proposição acima considere a seguinte distribuição conjunta.

$$
\begin{aligned}
F_{X_{1}, X_{1}+X_{2}}(s, t) & =P\left[X_{1} \leq s, X_{1}+X_{2} \leq t\right] \\
& =\int_{-\infty}^{s} \int_{-\infty}^{t} f_{X_{1}, X_{1}+X_{2}}(x, y) d x d y \\
& =\int_{-\infty}^{s} \int_{-\infty}^{t} f_{X_{1}+X_{2} \mid X_{1}}(y \mid x) f_{X_{1}}(x) d x d y \\
& =\int_{-\infty}^{s} F_{X_{1}+X_{2} \mid X_{1}}(t \mid x) f_{X_{1}}(x) d x \\
& =\int_{-\infty}^{s} P\left[X_{2} \leq t-x \mid X_{1}=x\right] d F_{X_{1}}(x)
\end{aligned}
$$

A partir do Lema 3 e utilizando a equação (1.19) com notação descrita por ela e substituindo $F_{X_{1}}(x)=u_{1}$ temos que,

$$
\begin{aligned}
F_{X_{1}, X_{1}+X_{2}}(s, t) & =\int_{-\infty}^{s} D_{1} C\left(F_{X_{1}}(x), F_{X_{2}}(t-x)\right) d F_{X_{1}}(x) \\
& =\int_{0}^{F_{X_{1}}(s)} D_{1} C\left(u_{1}, F_{X_{2}}\left(t-F_{X_{1}}^{-1}\left(u_{1}\right)\right)\right) d u_{1} .
\end{aligned}
$$

Assim, podemos obter a marginal $F_{X_{1}+X_{2}}$ realizando o passo descrito em (1.3), 
isto é, tomando o seguinte limite,

$$
\begin{aligned}
F_{X_{1}+X_{2}}(t) & =\lim _{s \rightarrow \infty} F_{X_{1}, X_{1}+X_{2}}(s, t) \\
& =\lim _{s \rightarrow \infty} \int_{0}^{F_{X_{1}}(s)} D_{1} C\left(u_{1}, F_{X_{2}}\left(t-F_{X_{1}}^{-1}\left(u_{1}\right)\right)\right) d u_{1} \\
& =\int_{0}^{1} D_{1} C\left(u_{1}, F_{X_{2}}\left(t-F_{X_{1}}^{-1}\left(u_{1}\right)\right)\right) d u_{1} .
\end{aligned}
$$

O mesmo raciocínio é válido para verificar (3.5), condicionando a $X_{2}$.

Uma consequência desta proposição é o Corolário 3 com formulação da função sobrevivência da soma de variáveis aleatória $X_{1}+X_{2}$.

Corolário 3 Sejam $X_{1}$ e $X_{2}$ duas variáveis aleatórias com mesmo espaço de probabilidade $(\Omega, \mathcal{F}, \boldsymbol{P})$ com estrutura de dependência dada pela cópula $C\left(u_{1}, u_{2}\right)$ e com marginais contínuas $F_{X_{1}}$ e $F_{X_{2}}$, então $\forall t \in \mathbb{R}$ tem-se quase certamente que,

$$
\bar{F}_{X_{1}+X_{2}}(t)=\int_{0}^{1} D_{1} C\left(u_{1}, \bar{F}_{X_{2}}\left(t-\bar{F}_{X_{1}}^{-1}\left(u_{1}\right)\right)\right) d u_{1}
$$

Prova. Por meio da equação (1.11) segue que,

$$
D_{1} \bar{C}\left(u_{1}, u_{2}\right)=1-D_{1} C\left(1-u_{1}, 1-u_{2}\right)
$$

Em virtude da Proposição 2 podemos reescrever (3.4) da seguinte forma,

$$
\begin{aligned}
\bar{F}_{X_{1}+X_{2}}(t) & =1-\int_{0}^{1} D_{1} C\left(u_{1}, F_{X_{2}}\left(t-F_{X_{1}}^{-1}\left(u_{1}\right)\right)\right) d u_{1} \\
& =1-\int_{0}^{1} 1-D_{1} \bar{C}\left(1-u_{1}, \bar{F}_{X_{2}}\left(t-F_{X_{1}}^{-1}\left(u_{1}\right)\right)\right) d u_{1} \\
& =1-\int_{0}^{1} 1-D_{1} \bar{C}\left(1-u_{1}, \bar{F}_{X_{2}}\left(t-\bar{F}_{X_{1}}^{-1}\left(1-u_{1}\right)\right)\right) d u_{1}
\end{aligned}
$$


substituindo $v_{1}=1-u_{1}$,

$$
\begin{aligned}
\bar{F}_{X_{1}+X_{2}}(t) & =-\int_{1}^{0} D_{1} \bar{C}\left(v_{1}, \bar{F}_{X_{2}}\left(t-\bar{F}_{X_{1}}^{-1}\left(v_{1}\right)\right)\right) d v_{1} \\
& =\int_{0}^{1} D_{1} \bar{C}\left(v_{1}, \bar{F}_{X_{2}}\left(t-\bar{F}_{X_{1}}^{-1}\left(v_{1}\right)\right)\right) d v_{1}
\end{aligned}
$$

Assim como $u_{1}, 0 \leq v_{1} \leq 1$, logo, troca-se $v_{1}$ por $u_{1}$, obtendo a expressão final.

Outro corolário interessante surge a partir da simetria radial definida na seção 1.3 .

Corolário 4 Se $\bar{C}\left(u_{1}, u_{2}\right)=C\left(u_{1}, u_{2}\right)$ e $\bar{F}_{X_{1}}(t)=F_{X_{1}}(-t)$ assim como $\bar{F}_{X_{2}}(t)=$ $F_{X_{2}}(-t)$, então a distribuição da soma também é simétrica em relação a 0 , isto é, $\bar{F}_{X_{1}+X_{2}}(t)=F_{X_{1}+X_{2}}(-t)$.

A prova é imediata a partir da definição de simetria radial.

Outra notação para (3.4) surge ao considerar $X_{1} \sim F, X_{2} \sim G$ e $C$ cópula associada a $\left(X_{1}, X_{2}\right)$, da seguinte forma,

$$
F_{*}^{C} G(t)=\int_{0}^{1} D_{1} C\left(u_{1}, G\left(t-F^{-1}\left(u_{1}\right)\right)\right) d u_{1},
$$

chamada de $C$-convolução. Se $F$ e $G$ são independentes, então a convolução é escrita por $F *{ }_{*}^{\Pi} G(t)$. Para ilustrar melhor, segue-se alguns exemplos de $C$ convoluções.

Exemplo 4 (П-Convolução). Se a função cópula $C=\Pi$, então a П-convolução de $X_{1}$ e $X_{2}$ coincide com a convolução clássica de variáveis aleatórias independentes.

$$
\begin{aligned}
F * G(t) & =\int_{0}^{1} D_{1} \Pi\left(u_{1}, G\left(t-F^{-1}\left(u_{1}\right)\right)\right) d u_{1}\left(D_{1} \Pi\left(u_{1}, u_{2}\right)=u_{2}\right) \\
& =\int_{0}^{1} G\left(t-F^{-1}\left(u_{1}\right)\right) d u_{1} \\
& =\int_{-\infty}^{+\infty} G\left(t-x_{1}\right) f\left(x_{1}\right) d x_{1}=\int_{-\infty}^{t} \int_{-\infty}^{+\infty} g\left(z-x_{1}\right) f\left(x_{1}\right) d x_{1} d z \\
& =F_{X_{1}+X_{2}}(t)
\end{aligned}
$$


ao considerar $D_{1} \Pi\left(u_{1}, u_{2}\right)=u_{2}$ e $x_{1}=F^{-1}\left(u_{1}\right)$.

Exemplo 5 ( $C_{U}$-Convolução). Se a função cópula $C=C_{U}$, então temos que,

$$
D_{1} C_{U}\left(u_{1}, u_{2}\right)= \begin{cases}1 & \text { se } u_{1} \leq u_{2} \\ 0 & \text { se } u_{1}>u_{2}\end{cases}
$$

A $C_{U}$-Convolução da distribuição $G$ e $F$ é,

$$
\begin{aligned}
F_{*}^{C_{U}} G(t) & =\int_{0}^{1} D_{1} C_{U}\left(u_{1}, G\left(t-F^{-1}\left(u_{1}\right)\right)\right) d u_{1} \\
& =\int_{0}^{1} \boldsymbol{I}_{\left[0, G\left(t-F^{-1}\left(u_{1}\right)\right)\right]}\left(u_{1}\right) d u_{1} \\
& =\int_{0}^{1} \boldsymbol{I}_{\left\{u_{1} \mid G^{-1}\left(u_{1}\right)+F^{-1}\left(u_{1}\right) \leq t\right\}}\left(u_{1}\right) d u_{1} \\
& =\sup \left\{u_{1} \in(0,1) \mid G^{-1}\left(u_{1}\right)+F^{-1}\left(u_{1}\right) \leq t\right\} .
\end{aligned}
$$

onde $\boldsymbol{I}$ é uma variável indicadora.

Para as cópulas Arquimedianas e utilizando a equação (1.41), a $C$-convolução de $F$ e $G$ fica expressa por,

$$
F \stackrel{C}{*} G(t)=\int_{0}^{1} \frac{\varphi^{\prime}\left(u_{1}\right)}{\varphi^{\prime}\left(\varphi^{-1}\left(\varphi\left(u_{1}\right)+\varphi\left(G\left(t-F^{-1}\left(u_{1}\right)\right)\right)\right)\right)} d u_{1}
$$

Para cada família de cópulas, a expressão da $C$-convolução é distinta. O Exemplo a seguir é um caso particular de (3.10).

Exemplo 6 (Clayton Convolução). Sejam $X_{1}$ e $X_{2}$ variáveis aleatórias com distribuição acumulada de uma exponencial $F$ e $G$ com parâmetro $\lambda_{F}$ e $\lambda_{G}$, respectivamente. Além disso, considere a cópula Clayton. Pela Tabela 1.1 a função geradora da Clayton é,

$$
\varphi_{\theta}(t)=\frac{1}{\theta}\left(t^{-\theta}-1\right)
$$

Em consequência,

$$
\varphi_{\theta}^{-1}(t)=-t^{-(\theta+1)}
$$


e pela equação (3.10),

$$
\begin{aligned}
F^{C} G(t) & =\int_{0}^{1}\left[u_{1}\left(u_{1}^{-\theta}+\left(G\left(t-F^{-1}\left(u_{1}\right)\right)\right)^{-\theta}-1\right)^{1 / \theta}\right]^{-(\theta+1)} d u_{1} \\
& =\int_{0}^{1}\left[u_{1}\left(u_{1}^{-\theta}+\left(1-\exp \left\{-\frac{\lambda_{G}}{\lambda_{F}}\left(\lambda_{F} t+\ln \left(1-u_{1}\right)\right)\right\}\right)^{-\theta}-1\right)^{1 / \theta}\right]^{-(\theta+1)} d u_{1}
\end{aligned}
$$

Calculando numericamente $F \stackrel{C}{*} G(t)$ e sua respectiva densidade $f \stackrel{C}{*} g(t)$, obtemos as curvas 3.1a e 3.1b no gráfico 3.1.

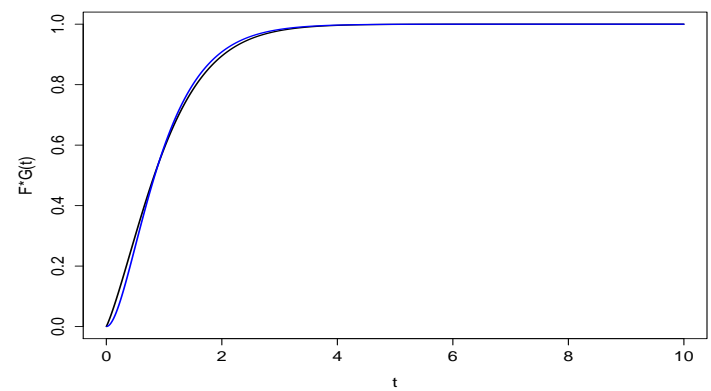

(a) $F \stackrel{C}{*} G(t)$

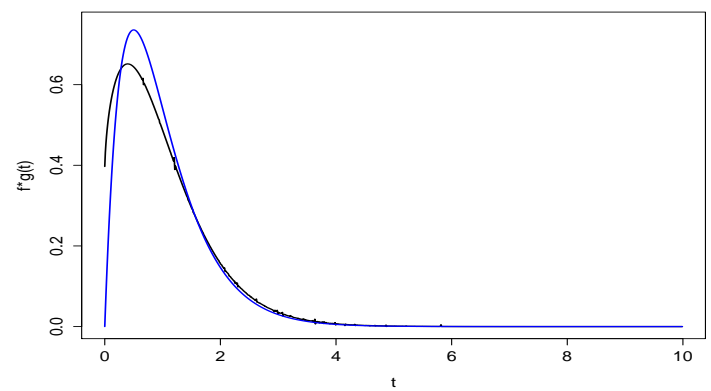

(b) $f \stackrel{C}{*} g(t)$

Figura 3.1: $C$-Convolução de uma Clayton, $\operatorname{com} \theta=0.5, \lambda_{F}=\lambda_{G}=2$ (preto) e distribuição Gama $W \sim \Gamma(2,2)$ (azul).

Quando o parâmetro da cópula de Clayton $\theta=0,5(\tau(C)=0,2)$, as variáveis ficam mais próximas da independência, sendo totalmente independente quando $\theta=0$. Desta forma, $F \stackrel{C}{*} G(t)$ aproxima-se de uma distribuição Gama, i.e. $\left(X_{1}+\right.$ $\left.X_{2}\right) \sim \Gamma(2,2)$ como consta na Figura 3.1. Aumentando o grau de dependência entre as variáveis, $\operatorname{com} \theta=2,3(\tau(C)=0,53)$, a distância com relação a distribuição Gama amplia-se. Este comportamento é explicitado pelas curvas na Figura 3.2. 


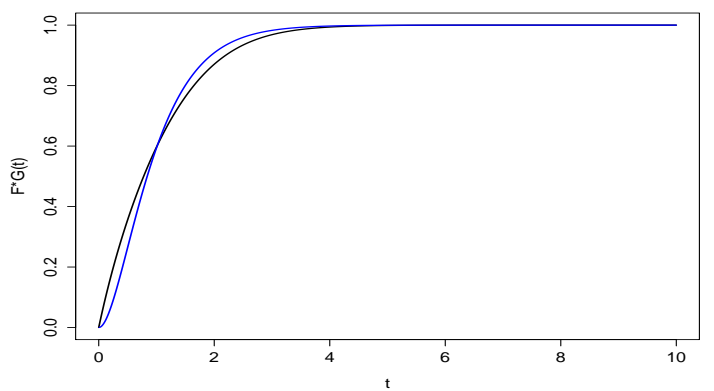

(a) $F \stackrel{C}{*} G(t)$

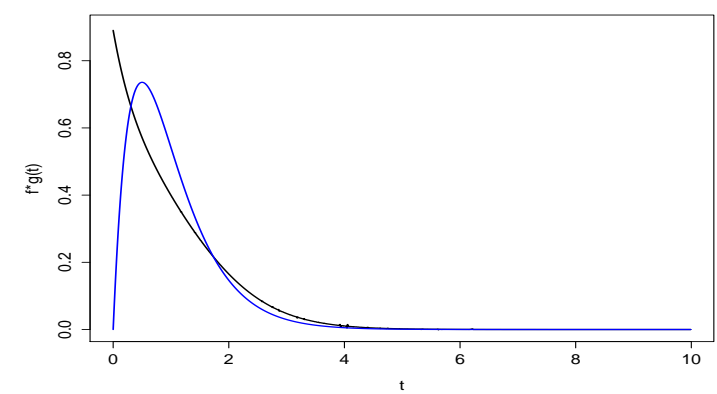

(b) $f \stackrel{C}{*} g(t)$

Figura 3.2: Convolução- $C$ de uma Clayton, $\operatorname{com} \theta=2, \lambda_{F}=2$ e $\lambda_{G}=2$ (preto) e distribuição Gama $W \sim \Gamma(2,2)$ (azul).

As curvas de níveis da cópula Clayton com parâmetros $\theta=0,5$ e $\theta=2,3$ podem ser vistas na Figura 1.8.

Como discutido na seção 1.6 no Capítulo 1, a construção de cópulas por meio de misturas permite gerar estruturas de dependências mais flexíveis. O resultado da distribuição da soma de $X_{1}+X_{2}$ quando a cópula associada a $\left(X_{1}, X_{1}\right)$ é uma mistura de cópula escrevemos o seguinte corolário.

Corolário 5 Sejam $X_{1}$ e $X_{2}$ duas variáveis aleatórias contínuas com mesmo espaço de probabilidade $(\Omega, \mathcal{F}, \boldsymbol{P})$. Sendo $C\left(u_{1}, u_{2}\right)=w C_{1}\left(u_{1}, u_{2}\right)+(1-w) C_{2}\left(u_{1}, u_{2}\right)$ a cópula associada as variáveis $X_{1}$ e $X_{2}$ gerada a partir da mistura das cópulas $C_{1}$ e $C_{2}$ onde $0 \leq w \leq 1$ e com marginais contínuas $F_{X_{1}}$ e $F_{X_{2}}$, então $\forall t \in \mathbb{R}$ quase certamente temos que a distribuição da soma $X_{1}+X_{2}$ com estrutura de dependência dada por $C$ é dada por,

$$
F_{X_{1}+X_{2}}(t)=w F_{X_{1}} \stackrel{C_{1}}{*} F_{X_{2}}(t)+(1-w) F_{X_{1}} \stackrel{C_{2}}{*} F_{X_{2}}(t) .
$$

A demonstração é simples e direta. 


\section{Prova.}

$$
\begin{aligned}
& F_{X_{1}+X_{2}}(t) \\
& =\int_{0}^{1} D_{1} C_{m}\left(u_{1}, F_{X_{2}}\left(t-F_{X_{1}}^{-1}\left(u_{1}\right)\right)\right) d u_{1}, \\
& =\int_{0}^{1} D_{1}\left[w C_{1}\left(u_{1}, F_{X_{2}}\left(t-F_{X_{1}}^{-1}\left(u_{1}\right)\right)\right)+(1-w) C_{2}\left(u_{1}, F_{X_{2}}\left(t-F_{X_{1}}^{-1}\left(u_{1}\right)\right)\right)\right] d u_{1}, \\
& =w \int_{0}^{1} D_{1} C_{1}\left(u_{1}, F_{X_{2}}\left(t-F_{X_{1}}^{-1}\left(u_{1}\right)\right)\right) d u_{1}+(1-w) \int_{0}^{1} D_{1} C_{2}\left(u_{1}, F_{X_{2}}\left(t-F_{X_{1}}^{-1}\left(u_{1}\right)\right)\right) d u_{1}, \\
& =w F^{C_{1}}{ }^{C_{1}} G(t)+(1-w) F_{*}^{C_{2}} G(t) .
\end{aligned}
$$

Em algumas situações estamos interessados não somente na distribuição da soma de duas variáveis, $X_{1}+X_{2}$, mas sim na distribuição de $X_{1}+\cdots+X_{n}$. Neste caso a formulação é análoga a $X_{1}+X_{2}$. Para obter a prova da distribuição dessa soma, seguindo os passos análogos aos da Proposição 2, primeiro escrevemos o resultado para $n=3$.

Proposição 3 Sejam $X_{1}, X_{2}$ e $X_{3}$ três variáveis aleatórias com mesmo espaço de probabilidade $(\Omega, \mathcal{F}, \boldsymbol{P})$. Sendo $C\left(u_{1}, u_{2}, u_{3}\right)$ a cópula associada as variáveis $X_{1}, X_{2}$ e $X_{3}$ com marginais contínuas $F_{X_{i}} i=1,2,3$, então $\forall t \in \mathbb{R}$ quase certamente temos que a distribuição da soma $W_{3}=X_{1}+X_{2}+X_{3}$ é dada por,

$$
F_{W_{3}}(t)=\int_{0}^{1} \int_{0}^{1} D_{1,2} C\left(u_{1}, u_{2}, F_{X_{3}}\left(t-F_{X_{1}}^{-1}\left(u_{1}\right)-F_{X_{2}}^{-1}\left(u_{2}\right)\right)\right) d u_{1} d u_{2},
$$

também de forma equivalente,

$$
F_{W_{3}}(t)=\int_{0}^{1} \int_{0}^{1} D_{2,3} C\left(F_{X_{1}}\left(t-F_{X_{2}}^{-1}\left(u_{2}\right)-F_{X_{3}}^{-1}\left(u_{3}\right)\right), u_{2}, u_{3}\right) d u_{2} d u_{3}
$$


De fato:

$$
\begin{aligned}
F_{X_{1}, X_{2}, W_{3}}(r, s, t) & =P\left(X_{1} \leq r, X_{2} \leq s, W_{3} \leq t\right), \\
& =\int_{-\infty}^{r} \int_{-\infty}^{s} \int_{-\infty}^{t} f_{X_{1}, X_{2}, W_{3}}\left(x_{1}, x_{2}, x_{3}\right) d x_{1} d x_{2} d x_{3} .
\end{aligned}
$$

Condicionando a $x_{1}$ e $x_{2}$ (Lei da Probabilidade Total) temos,

$$
\begin{aligned}
& F_{X_{1}, X_{2}, W_{3}}(r, s, t) \\
& =\int_{-\infty}^{r} \int_{-\infty}^{s} \int_{-\infty}^{t} f_{W_{3} \mid X_{1}, X_{2}}\left(x_{3} \mid x_{1}, x_{2}\right) f_{X_{1}, X_{2}}\left(x_{1}, x_{2}\right) d x_{1} d x_{2} d x_{3} \\
& =\int_{-\infty}^{r} \int_{-\infty}^{s} F_{W_{3} \mid X_{1}, X_{2}}\left(t \mid x_{1}, x_{2}\right) f_{X_{1}, X_{2}}\left(x_{1}, x_{2}\right) d x_{1} d x_{2} \\
& =\int_{-\infty}^{r} \int_{-\infty}^{s} P\left(X_{3} \leq t-x_{1}-x_{2} \mid X_{1}=x_{1}, X_{2}=x_{2}\right) f_{X_{1}, X_{2}}\left(x_{1}, x_{2}\right) d x_{1} d x_{2}
\end{aligned}
$$

Tomando o limite $r \uparrow \infty$ e $s \uparrow \infty$, temos a distribuição de $W_{3}$. Porém, podemos reescrever esta última passagem da equação acima utilizando a eq.(1.18) exposta na seção 1.3.2 do Capítulo 1. Desta forma, fazendo a substituição $u_{i}=F_{X_{i}}\left(x_{i}\right)$, $i=1,2,3$ temos que,

$$
\begin{aligned}
F_{W_{3}}(t) & =\int_{0}^{1} \int_{0}^{1} P\left(U_{3} \leq F_{X_{3}}\left(t-F_{X_{1}}^{-1}\left(u_{1}\right)-F_{X_{2}}^{-1}\left(u_{2}\right)\right) \mid U_{1}=u_{1}, U_{2}=u_{2}\right) \\
& \times c_{12}\left(u_{1}, u_{2}\right) d u_{1} d u_{2} .
\end{aligned}
$$

A partir da relação descrita por (1.22) tem-se,

$$
F_{W_{3}}(t)=\int_{0}^{1} \int_{0}^{1} \frac{D_{1,2} C\left(u_{1}, u_{2}, u_{3}\right)}{D_{1,2} C\left(u_{1}, u_{2}, 1\right)} c_{12}\left(u_{1}, u_{2}\right) d u_{1} d u_{2}
$$

e substituindo $u_{3}=t-F_{X_{1}}^{-1}\left(u_{1}\right)-F_{X_{2}}^{-1}\left(u_{2}\right)$ temos o resultado final,

$$
F_{W_{3}}(t)=\int_{0}^{1} \int_{0}^{1} D_{1,2} C\left(u_{1}, u_{2}, F_{X_{3}}\left(t-F_{X_{1}}^{-1}\left(u_{1}\right)-F_{X_{2}}^{-1}\left(u_{2}\right)\right)\right) d u_{1} d u_{2} .
$$


A generalização para a soma $\sum_{i=1}^{n} X_{i}$, em que os $X_{i}$ são todas contínuas, segue na proposição abaixo.

Proposição 4 Sejam $X_{1}, X_{2}, \ldots, X_{n}$ variáveis aleatórias com mesmo espaço de probabilidade $(\Omega, \mathcal{F}, \boldsymbol{P})$. Sendo $C\left(u_{1}, u_{2}, \cdots, u_{3}\right)$ a cópula associada ao vetor de variáveis aleatórias $\left(X_{1}, X_{2}, \cdots, X_{n}\right)$ com marginais contínuas $F_{X_{i}} i=1,2, \ldots, n$, então $\forall t \in \mathbb{R}$ quase certamente temos que a distribuição da soma $W_{n}=\sum_{i=1}^{n} X_{i}$ é dada por,

$$
F_{W_{n}}(t)=\int_{0}^{1} \cdots \int_{0}^{1} D_{1: n-1} C\left(u_{1}, \ldots, u_{n-1}, F_{X_{n}}\left(t-\sum_{i=1}^{n-1} F_{X_{i}}^{-1}\left(u_{i}\right)\right)\right) d u_{1} \cdots d u_{n-1}
$$

ou de forma equivalente,

$$
F_{W_{n}}(t)=\int_{0}^{1} \cdots \int_{0}^{1} D_{2: n} C\left(F_{X_{1}}\left(t-\sum_{i=2}^{n} F_{X_{i}}^{-1}\left(u_{i}\right)\right), u_{2}, u_{3}, \ldots, u_{n}\right) d u_{2} d u_{3} \cdots d u_{n}
$$

Prova. Seguiremos a prova por indução ${ }^{1}$. Seja $W_{n}=\sum_{i=1}^{n} X_{i}$ em que $X_{i}, i=$ $1,2, \cdots, n$ são variáveis aleatórias no mesmo espaço de probabilidade $(\Omega, \mathcal{F}, \boldsymbol{P})$. Pela Proposição 2 verificamos que para $n=2$,

$$
F_{W_{2}}(t)=\int_{0}^{1} D_{2} C\left(F_{X_{1}}\left(t-F_{X_{2}}^{-1}\left(u_{2}\right)\right), u_{2}\right) d u_{2}
$$

é verdadeira. Suponhamos agora que para algum $n \in \mathbb{N}$ tem-se que $F_{W_{n}}(t)$, formulado por (3.16), também seja válido. Compondo $W_{n+1}=W_{n}+X_{n+1}$ a distribuição

\footnotetext{
${ }^{1} \mathrm{O}$ teorema sobre indução matemática se encontra no apêndice.
} 
para $n+1$ variáveis fica,

$$
\begin{aligned}
F_{X_{n+1}, W_{n+1}}(s, t) & =P\left(X_{n+1} \leq s, W_{n+1} \leq t\right) \\
& =\int_{-\infty}^{s} \int_{-\infty}^{t} f_{X_{n+1}, W_{n+1}}\left(x_{n+1}, w\right) d x_{n+1} d w \\
& =\int_{-\infty}^{s} \int_{-\infty}^{t} f_{W_{n+1} \mid X_{n+1}}\left(w \mid x_{n+1}\right) f_{X_{n+1}}\left(x_{n+1}\right) d w d x_{n+1} \\
& =\int_{-\infty}^{s} F_{W_{n+1} \mid X_{n+1}}\left(t \mid x_{n+1}\right) f_{X_{n+1}}\left(x_{n+1}\right) d x_{n+1} \\
& =\int_{-\infty}^{s} P\left(W_{n} \leq t-x_{n+1} \mid X_{n+1}=x_{n+1}\right) f_{X_{n+1}}\left(x_{n+1}\right) d x_{n+1} .
\end{aligned}
$$

Usando a lei da probabilidade total segue que,

$$
\begin{aligned}
F_{X_{n+1}, W_{n+1}}(s, t) & =\int_{-\infty}^{s} \int_{-\infty}^{\infty} \cdots \int_{-\infty}^{\infty} P\left(X_{1} \leq t-x_{2} \cdots-x_{n+1} \mid X_{2}=x_{2}, \ldots, X_{n+1}=x_{n+1}\right) \\
& \times f_{X_{2} \cdots X_{n+1}}\left(x_{2}, \ldots, x_{n}, x_{n+1}\right) d x_{2} \cdots d x_{n+1},
\end{aligned}
$$

com a substituição, $u_{i}=F_{X_{i}}\left(x_{i}\right)$ para $i=1, \ldots, n+1$ temos,

$$
\begin{aligned}
& F_{X_{n+1}, W_{n+1}}(s, t) \\
& =\int_{0}^{F_{X_{n+1}}(s)} \int_{0}^{1} \cdots \int_{0}^{1} P\left(U_{1} \leq F_{X_{1}}\left(t-F_{X_{2}}^{-1}\left(u_{2}\right) \cdots-F_{X_{n+1}}^{-1}\left(u_{n+1}\right)\right) \mid U_{2}=u_{2}, \cdots, U_{n+1}=u_{n+1}\right) \\
& \times c\left(u_{2}, \cdots, u_{n+1}\right) d u_{2} \cdots d u_{n+1} .
\end{aligned}
$$

Para obter a expressão da distribuição de $U_{1}$ dado $U_{2}, \cdots, U_{n+1}$ utilizamos a relação descrita por (1.23).

$$
\begin{aligned}
F_{X_{n+1}, W_{n+1}}(s, t) & =\int_{0}^{F_{X_{n+1}}(s)} \int_{0}^{1} \cdots \int_{0}^{1} \frac{D_{2: n+1} C\left(u_{1}, \cdots, u_{n+1}\right)}{D_{2: n+1} C\left(u_{2}, \cdots, u_{n+1}\right)} c\left(u_{2}, \cdots, u_{n+1}\right) d u_{2} \cdots d u_{n+1} \\
& =\int_{0}^{F_{X_{n+1}}(s)} \int_{0}^{1} \cdots \int_{0}^{1} D_{2: n+1} C\left(F_{X_{1}}\left(t-\sum_{i=2}^{n+1} F_{X_{i}}^{-1}\left(u_{i}\right)\right)\right) d u_{2} \cdots d u_{n+1} .
\end{aligned}
$$

Agora, aplicando o limite $s \uparrow \infty$,

$$
F_{W_{n+1}}(t)=\int_{0}^{1} \int_{0}^{1} \cdots \int_{0}^{1} D_{2: n+1} C\left(F_{X_{1}}\left(t-\sum_{i=2}^{n+1} F_{X_{i}}^{-1}\left(u_{i}\right)\right), u_{2}, \cdots, u_{n+1}\right) d u_{2} \cdots d u_{n+1} .
$$


Exemplo 7 Se $X_{1}, X_{2}, X_{3}$ são v.a's com cópula associada $C=\Pi$ então,

$$
C\left(u_{1}, u_{2}, u_{3}\right)=u_{1} u_{2} u_{3}
$$

e

$$
D_{12} C\left(u_{1}, u_{2}, u_{3}\right)=u_{3}
$$

Utilizando (3.12),

$$
\begin{aligned}
F_{X_{1}+X_{2}+X_{3}}(t) & =\int_{0}^{1} \int_{0}^{1} D_{12} C\left(u_{1}, u_{2}, F_{X_{3}}\left(t-F_{X_{1}}^{-1}\left(u_{1}\right)-F_{X_{2}}^{-1}\left(u_{2}\right)\right)\right) d u_{1} d u_{2} \\
& =\int_{0}^{1} \int_{0}^{1} F_{X_{3}}\left(t-F_{X_{1}}^{-1}\left(u_{1}\right)-F_{X_{2}}^{-1}\left(u_{2}\right)\right) d u_{1} d u_{2} \\
& =\int_{-\infty}^{\infty} \int_{-\infty}^{\infty} F_{X_{3}}\left(t-x_{1}-x_{2}\right) d F_{X_{1}}\left(x_{1}\right) d F_{X_{2}}\left(x_{2}\right) \\
& =\left(F_{X_{3}} * F_{X_{2}} * F_{X_{1}}\right)(t) .
\end{aligned}
$$

No âmbito de finanças e na gestão de riscos a variável aleatória $X_{i}$ representa o retorno de um determinado ativo $i$. Assim sendo, a combinação convexa $W_{2}=$ $\pi X_{1}+(1-\pi) X_{2}$ retrata o retorno total de uma carteira de investimento onde $0 \leq \pi \leq 1$ e designa o peso do ativo $i$ na carteira. Daí o interesse em determinar a distribuição de $W_{2}$, porém introduzido um peso $w$.

Corolário 6 Sejam $X_{1}$ e $X_{2}$ duas variáveis aleatórias contínuas com mesmo espaço de probabilidade $(\Omega, \mathcal{F}, \boldsymbol{P})$. Sendo $C\left(u_{1}, u_{2}\right)$ a cópula associada as variáveis $X_{1}$ e $X_{2}$ com marginais contínuas $F_{X_{1}}$ e $F_{X_{2}}$ e $0 \leq \pi \leq 1$, então $\forall t \in \mathbb{R}$ quase certamente temos que a distribuição da soma $\pi X_{1}+(1-\pi) X_{2}$ é dada por,

$$
F_{\pi X_{1}+(1-\pi) X_{2}}(t)=\int_{0}^{1} D_{1} C\left(u_{1}, F_{X_{2}}\left(\frac{t}{1-\pi}-\frac{\pi}{1-\pi} F_{X_{1}}^{-1}\left(u_{1}\right)\right)\right) d u_{1}
$$




\section{Prova.}

$$
\begin{aligned}
F_{\pi X_{1}, \pi X_{1}+(1-\pi) X_{2}}(s, t) & =P\left(\pi X_{1} \leq s, \pi X_{1}+(1-\pi) X_{2} \leq t\right) \\
& =\int_{-\infty}^{s} \int_{-\infty}^{t} f_{\pi X_{1}, \pi X_{1}+(1-\pi) X_{2}}(y, w) d y d w \\
& =\int_{-\infty}^{s} \int_{-\infty}^{t} f_{\pi X_{1}+(1-\pi) X_{2} \mid \pi X_{1}}(y, w) f_{\pi X_{1}}(y) d y d w
\end{aligned}
$$

Fazendo $y=\pi x$ temos,

$$
\begin{aligned}
F_{\pi X_{1}, \pi X_{1}+(1-\pi) X_{2}}(s, t) & =\int_{-\infty}^{s} F_{\pi X_{1}+(1-\pi) X_{2} \mid \pi X_{1}}(t \mid y) f_{\pi X_{1}}(y) d y \\
& =\int_{-\infty}^{s / \pi} P\left(X_{2} \leq \frac{t-\pi X_{1}}{1-\pi} \mid X_{1}=x\right) d F_{X_{1}}(x) .
\end{aligned}
$$

Pela equação (1.19) e pela substituição $F_{X_{1}}(x)=u_{1}$ tem-se,

$$
\begin{aligned}
F_{\pi X_{1}, \pi X_{1}+(1-\pi) X_{2}}(s, t) & =\int_{0}^{F_{X_{1}}(s / \pi)} D_{1} C\left(F_{X_{1}}(x), F_{X_{2}}\left(\frac{t-\pi x}{1-\pi}\right)\right) d F_{X_{1}}(x) \\
& =\int_{0}^{F_{X_{1}}(s / \pi)} D_{1} C\left(u_{1}, F_{X_{2}}\left(\frac{t}{1-\pi}-\frac{\pi}{1-\pi} F_{X_{1}}^{-1}\left(u_{1}\right)\right)\right) d u_{1}
\end{aligned}
$$

O resultado (3.17) segue da última equação ao aplicar o limite $s \uparrow \infty$.

Para um portfólio com $n$ ativos, o retorno total é dado pela combinação convexa desses ativos e sua distribuição é formulação geral de (3.17), dada no seguinte corolário. Generalizando o caso de um portfólio $W_{n}$ com $n \in \mathbb{N}$, temos que o retorno total dado pela combinação convexa dos $n$ ativos pode ser formulado nos mesmos moldes que o caso $n=2$, como segue o Corolário abaixo.

Corolário 7 Sejam $X_{i}, i=1, \ldots, n$ variáveis aleatórias contínuas com mesmo espaço de probabilidade $(\Omega, \mathcal{F}, \boldsymbol{P})$. Sendo $C\left(u_{1}, \ldots, u_{n}\right)$ a cópula associada a essas variáveis aleatórias com marginais contínuas $F_{X_{i}}$ então $\forall t \in \mathbb{R}$ quase certamente temos que a distribuição da soma $W_{n}=\sum_{i=1}^{n} \pi_{i} X_{i} \operatorname{com} \sum_{i=1}^{n} \pi_{i}=1$ onde $\pi_{i} \geq 0$ é dada por,

$$
F_{W_{n}}(t)=\int_{0}^{1} \cdots \int_{0}^{1} D_{1: n-1} C\left(u_{1}, \ldots, u_{n-1}, F_{X_{n}}\left(\pi_{n}^{-1}\left(t-\sum_{i=1}^{n-1} \pi_{i} F_{X_{i}}^{-1}\left(u_{i}\right)\right)\right)\right) d u_{1} \cdots d u_{n-1} .
$$


A prova do Corolário 7 segue a mesma estrutura da demonstração da Proposição 4 .

\subsection{Distribuição do produto e do quociente}

$\mathrm{Na}$ seção anterior foram introduzidas as formulações das distribuições nos casos da soma de variáveis aleatórias $W_{n}=\sum_{i=1}^{n} X_{i}$ para $n \in \mathbb{N}$ e da combinação convexa entre os $X_{i}$ 's, quando temos uma estrutura de dependência. Estas distribuições possuem interesse nas áreas como finanças e atuária. Funções de distribuição para o produto $X_{1} X_{2}$ e para os quocientes $X_{2} / X_{1}$ e $X_{1} /\left(X_{1}+X_{2}\right)$ também são estudadas em várias áreas. Alguns exemplos dessas formulações com estruturas de dependência específicas podem ser vista em Nadarajah (2005a), Nadarajah (2005b), Nadarajah e Ali (2006) e Nagar et al. (2009). Expressões para o produto e razão considerando uma estrutura de dependência geral, dada por um função cópula $C$, é introduzida nesta seção. A seguinte proposição enuncia a expressão geral para o caso de $X_{2} / X_{1}$ e $X_{1} X_{2}$.

Proposição 5 Seja $\left(X_{1}, X_{2}\right)$ um vetor de variáveis aleatórias definido sob o mesmo espaço de probabilidade $(\mathbb{R}, \mathcal{F}, \boldsymbol{P})$. Sendo $C\left(u_{1}, u_{2}\right)$ a cópula associada ao vetor $\left(X_{1}, X_{2}\right)$ com marginais contínuas $F_{X_{1}}$ e $F_{X_{2}}$, então $\forall t \in \mathbb{R}$ quase certamente temos que as distribuições de $X_{2} / X_{1}$ e de $X_{1} X_{2}$ são dadas respectivamente por,

$$
\begin{aligned}
F_{X_{2} / X_{1}}(t) & =F_{X_{1}}(0)-\int_{0}^{F_{X_{1}}(0)} D_{1} C\left(u_{1}, F_{X_{2}}\left(t F_{X_{1}}^{-1}\left(u_{1}\right)\right)\right) d u_{1} \\
& +\int_{F_{X_{1}}(0)}^{1} D_{1} C\left(u_{1}, F_{X_{2}}\left(t F_{X_{1}}^{-1}\left(u_{1}\right)\right)\right) d u_{1}, \\
F_{X_{2} X_{1}}(t) & =F_{X_{1}}(0)-\int_{0}^{F_{X_{1}}(0)} D_{1} C\left(u_{1}, F_{X_{2}}\left(t / F_{X_{1}}^{-1}\left(u_{1}\right)\right)\right) d u_{1} \\
& +\int_{F_{X_{1}}(0)}^{1} D_{1} C\left(u_{1}, F_{X_{2}}\left(t / F_{X_{1}}^{-1}\left(u_{1}\right)\right)\right) d u_{1} .
\end{aligned}
$$


A demonstração da distribuição do produto é análogo ao do quociente. Desta forma, será suficiente apresentar a prova para o quociente.

\section{Prova.}

$$
\begin{aligned}
F_{X_{1}, X_{2} / X_{1}}(s, t) & =P\left(X_{1} \leq s, X_{2} / X_{1} \leq t\right) \\
& =\int_{-\infty}^{s} \int_{-\infty}^{t} f_{X_{1}, X_{2} / X_{1}}(z, w) d z d w \\
& =\int_{-\infty}^{s} \int_{-\infty}^{t} f_{X_{2} / X_{1} \mid X_{1}}(w \mid z) f_{X_{1}}(z) d w d z \\
& =\int_{-\infty}^{s} F_{X_{2} / X_{1} \mid X_{1}}(t \mid z) f_{X_{1}}(z) d z \\
& =\int_{-\infty}^{s} P\left(X_{2} / z \leq t \mid X_{1}=z\right) d F_{X_{1}} .
\end{aligned}
$$

Em (3.21), para isolar $X_{1}$, dividimos o suporte de $z$ em duas partes. Assim, para $X_{1}=z<0$ temos,

$$
F_{X_{1}, X_{2} / X_{1}}(s, t)=\int_{-\infty}^{s} P\left(X_{2}>t z \mid X_{1}=z\right) d F_{X_{1}}
$$

e para $X_{1}=z \geq 0$

$$
F_{X_{1}, X_{2} / X_{1}}(s, t)=\int_{0}^{s} P\left(X_{2} \leq t z \mid X_{1}=z\right) d F_{X_{1}}
$$

Substituindo (3.23) e (3.22) em (3.21) a função $F_{X_{1}, X_{2} / X_{1}}(s, t)$ é reescrita da seguinte forma,

$$
F_{X_{1}, X_{2} / X_{1}}(s, t)= \begin{cases}\int_{-\infty}^{s} P\left(X_{2}>t z \mid X_{1}=z\right) d F_{X_{1}}, & \text { se } s \leq 0 \\ \int_{-\infty}^{0} P\left(X_{2}>t z \mid X_{1}=z\right) d F_{X_{1}}+\int_{0}^{s} P\left(X_{2} \leq t z \mid X_{1}=z\right) d F_{X_{1}}, & \text { se } s>0 .\end{cases}
$$


Aplicando o limite quando $s \uparrow \infty$ em (3.24) obtemos,

$$
\begin{aligned}
F_{X_{2} / X_{1}}(t) & =\lim _{s \rightarrow \infty} F_{X_{1}, X_{2} / X_{1}}(s, t) \\
& =\int_{-\infty}^{0} P\left(X_{2}>t z \mid X_{1}=z\right) d F_{X_{1}}+\int_{0}^{\infty} P\left(X_{2} \leq t z \mid X_{1}=z\right) d F_{X_{1}} \\
& =\int_{-\infty}^{0}\left[1-P\left(X_{2} \leq t z \mid X_{1}=z\right)\right] d F_{X_{1}}+\int_{0}^{\infty} P\left(X_{2} \leq t z \mid X_{1}=z\right) d F_{X_{1}} \\
& =\int_{-\infty}^{0} d F_{X_{1}}-\int_{-\infty}^{0} P\left(X_{2} \leq t z \mid X_{1}=z\right) d F_{X_{1}}+\int_{0}^{\infty} P\left(X_{2} \leq t z \mid X_{1}=z\right) d F_{X_{1}} .
\end{aligned}
$$

Agora, substituindo $u_{1}=F_{X_{1}}(z)$ e aplicando (1.19),

$$
\begin{aligned}
F_{X_{2} / X_{1}}(t)= & F_{X_{1}}(0)-\int_{0}^{F_{X_{1}}(0)} D_{1} C\left(u_{1}, F_{X_{2}}\left(t F_{X_{1}}^{-1}\left(u_{1}\right)\right)\right) d u_{1} \\
& +\int_{F_{X_{1}}(0)}^{1} D_{1} C\left(u_{1}, F_{X_{2}}\left(t F_{X_{1}}^{-1}\left(u_{1}\right)\right)\right) d u_{1}
\end{aligned}
$$

A partir das mesmas condições da Proposição 5 podemos estabelecer algumas consequências imediatas das expressões (3.19) e (3.20). Observe que se as variáveis aleatórias $X_{1}$ e $X_{2}$ são não negativas, então a distribuição do quociente tipo $1 \mathrm{e}$ do produto é dada respectivamente por,

$$
\begin{aligned}
F_{X_{2} / X_{1}}(t) & =\int_{0}^{1} D_{1} C\left(u_{1}, F_{X_{2}}\left(t F_{X_{1}}^{-1}\left(u_{1}\right)\right)\right) d u_{1} \\
F_{X_{2} X_{1}}(t) & =\int_{0}^{1} D_{1} C\left(u_{1}, F_{X_{2}}\left(t / F_{X_{1}}^{-1}\left(u_{1}\right)\right)\right) d u_{1}
\end{aligned}
$$

A função densidade de $X_{2} / X_{1}$ e $X_{2} X_{1}$ para estes mesmos casos são,

$$
\begin{aligned}
f_{X_{2} / X_{1}}(t) & =\int_{0}^{1} c\left(u_{1}, F_{X_{2}}\left(t F_{X_{1}}^{-1}\left(u_{1}\right)\right)\right) f_{X_{2}}\left(t F_{X_{1}}^{-1}\left(u_{1}\right)\right) F_{X_{1}}^{-1}\left(u_{1}\right) d u_{1}, \\
f_{X_{2} X_{1}}(t) & =\int_{0}^{1} c\left(u_{1}, F_{X_{2}}\left(t / F_{X_{1}}^{-1}\left(u_{1}\right)\right)\right) f_{X_{2}}\left(t / F_{X_{1}}^{-1}\left(u_{1}\right)\right)\left(F_{X_{1}}^{-1}\left(u_{1}\right)\right)^{-1} d u_{1} .
\end{aligned}
$$

Sendo a função cópula associada as variáveis aleatórias, $X_{1}$ e $X_{2}$ da família Arquimediana, então a distribuição de $X_{1} / X_{2}$ e $X_{1} X_{2}$ ficam expressas respectivamente 
94 Capítulo 3. Distribuição de Funções de Variáveis Aleatórias via Funções Cópulas

por,

$$
\begin{aligned}
F_{X_{2} / X_{1}}(t) & =\int_{0}^{1} \frac{\varphi^{\prime}\left(u_{1}\right)}{\varphi^{\prime}\left(\varphi^{-1}\left(\varphi\left(u_{1}\right)+\varphi\left(F_{X_{2}}\left(t F_{X_{1}}^{-1}\left(u_{1}\right)\right)\right)\right)\right)} d u_{1}, \\
F_{X_{2} X_{1}}(t) & =\int_{0}^{1} \frac{\varphi^{\prime}\left(u_{1}\right)}{\varphi^{\prime}\left(\varphi^{-1}\left(\varphi\left(u_{1}\right)+\varphi\left(F_{X_{2}}\left(t / F_{X_{1}}^{-1}\left(u_{1}\right)\right)\right)\right)\right)} d u_{1} .
\end{aligned}
$$

Uma função de interesse na área de hidrologia e que aparece recorrentemente na literatura é o quociente $\frac{X_{1}}{X_{1}+X_{2}}$. Na seguinte proposição exibimos a expressão para a distribuição do quociente tipo 2 .

Proposição 6 Sejam $X_{1}$ e $X_{2}$ duas variáveis aleatórias contínuas não negativas com mesmo espaço de probabilidade $\left(\mathbb{R}^{+}, \mathcal{F}, \boldsymbol{P}\right)$. Sendo $C\left(u_{1}, u_{2}\right)$ a cópula associada as variáveis $X_{1}$ e $X_{2}$ com marginais contínuas $F_{X_{1}}$ e $F_{X_{2}}$, respectivamente, tem-se que a função de distribuição de $\frac{X_{1}}{X_{1}+X_{2}}$ é dada por,

$$
F_{\frac{X_{1}}{X_{1}+X_{2}}}(t)=1-\int_{0}^{1} D_{1} C\left(u_{1}, F_{X_{2}}\left(\frac{1-t}{t} F_{X_{1}}^{-1}\left(u_{1}\right)\right)\right) d u_{1} .
$$

\section{Prova.}

$$
\begin{aligned}
F_{X_{1}, X_{1} / X_{1}+X_{2}}(s, t) & =P\left(X_{1} \leq s, \frac{X_{1}}{X_{1}+X_{2}} \leq t\right) \\
& =\int_{0}^{s} \int_{-\infty}^{t} f_{X_{1}, X_{1} / X_{1}+X_{2}}(z, w) d z d w \\
& =\int_{0}^{s} \int_{-\infty}^{t} f_{X_{1} / X_{1}+X_{2} \mid X_{1}}(w \mid z) f_{X_{1}}(z) d w d z \\
& =\int_{0}^{s} F_{X_{1} / X_{1}+X_{2} \mid X_{1}}(t \mid z) f_{X_{1}}(z) d z \\
& =\int_{0}^{s} P\left(\frac{z}{z+X_{2}} \leq t \mid X_{1}=z\right) d F_{X_{1}} \\
& =\int_{0}^{s} P\left(X_{2} \geq z \frac{1-t}{t} \mid X_{1}=z\right) d F_{X_{1}}
\end{aligned}
$$


Aplicando o limite quando $s \uparrow \infty$ em (3.30),

$$
\begin{aligned}
F_{X_{1} / X_{1}+X_{2}}(t) & =\lim _{s \rightarrow \infty} F_{X_{1}, X_{1} / X_{1}+X_{2}}(s, t) \\
& =\int_{0}^{\infty} P\left(X_{2} \geq z \frac{1-t}{t} \mid X_{1}=z\right) d F_{X_{1}} \\
& =\int_{0}^{\infty} 1-P\left(X_{2}<z \frac{1-t}{t} \mid X_{1}=z\right) d F_{X_{1}} .
\end{aligned}
$$

Substituindo $u_{1}=F_{X_{1}}(z)$ e utilizando a relação (1.19) obtemos a expressão final,

$$
F_{\frac{X_{1}}{X_{1}+X_{2}}}(t)=1-\int_{0}^{1} D_{1} C\left(u_{1}, F_{X_{2}}\left(\frac{1-t}{t} F_{X_{1}}^{-1}\left(u_{1}\right)\right)\right) d u_{1}
$$

A função densidade de $\frac{X_{1}}{X_{1}+X_{2}}$ é dada por,

$$
f_{\frac{X_{1}}{X_{1}+X_{2}}}(t)=\int_{0}^{1} c\left(u_{1}, F_{X_{2}}\left(\frac{1-t}{t} F_{X_{1}}^{-1}\left(u_{1}\right)\right)\right) f_{X_{1}}\left(\frac{1-t}{t} F_{X_{1}}^{-1}\left(u_{1}\right)\right) F_{X_{1}}^{-1}\left(u_{1}\right) \frac{1}{t^{2}} d u_{1} .
$$

Se ainda a função cópula associada as variáveis aleatórias $X_{1}$ e $X_{2}$ é da família Arquimediana, então a distribuição de $\frac{X_{1}}{X_{1}+X_{2}}$ fica expressa por,

$$
F_{\frac{X_{1}}{X_{1}+X_{2}}}(t)=1-\int_{0}^{1} \frac{\varphi^{\prime}\left(u_{1}\right)}{\varphi^{\prime}\left(\varphi^{-1}\left(\varphi\left(u_{1}\right)+\varphi\left(F_{X_{2}}\left(\frac{1-t}{t} F_{X_{1}}^{-1}\left(u_{1}\right)\right)\right)\right)\right)} d u_{1} .
$$





\section{Capítulo 4}

\section{Distribuição da Soma com Modelos}

\section{AR-GARCH}

No contexto de finanças, considera-se os retornos de $n$ ativos de uma carteira como variáveis aleatórias. Devido a exposição ao risco em que os ativos se encontram, existe uma dependência entre eles. A distribuição da soma, em particular a combinação convexa, representa o retorno de um portfólio. Uma vez definida a distribuição de $W_{n}=\sum_{i=1}^{n} \pi_{i} X_{i}$, estamos aptos a mensurar as medidas de risco do portfólio como o Value-at-Risk (VaR) e Expected Shortfalls $\left(\mathrm{ES}^{1}\right)$. Podemos ainda otimizar uma carteira definindo os valores $\pi_{i}$ tais que minimizem alguma medida de risco.

No entanto, como destaca Cont (2001), os fatos estilizados em finanças apontam que as séries temporais financeiras apresentam clusters de volatilidade. Assim, além da dependência dos retornos entre os ativos, a volatilidade dos retornos também possuem dependência serial. Para incorporar esta característica, vamos inserir uma estrutura GARCH em cada variável.

Neste capítulo apresentamos a distribuição da soma de variáveis aleatórias, governadas por um processo GARCH, em que as inovações possuem interdependência. Introduzimos inicialmente a construção da distribuição multivariada por

\footnotetext{
${ }^{1}$ A definição do ES encontra-se no Apêndice A
} 
meio das D-Vines Cópulas, porém com estrutura $\operatorname{GARCH}(\mathrm{p}, \mathrm{q})$ para cada distribuição marginal de $X_{i t}$. Mediante esta distribuição multivariada formulamos a distribuição da soma para $X_{1 t}+X_{2 t}$. Na sequência, apresentamos o caso geral da distribuição $X_{1 t}+X_{2 t}+\cdots+X_{n t}$. Por fim, ampliamos o caso da distribuição da soma para um processo AR-GARCH e também para sua combinação convexa. Com estas formulações, o cálculo do VaR torna-se dinâmico, isto é, para cada tempo temos uma valor para o Value-at-Risk.

Seja $\boldsymbol{X}_{t}=\left(X_{1 t}, X_{2 t}, \ldots, X_{n t}\right)$ um vetor aleatório $n$-dimensional para cada $i=1,2, \ldots, n$ e tempo $t=1,2, \ldots T$, tem-se que,

$$
\begin{aligned}
X_{i t} & =h_{i t}^{1 / 2} Y_{i t} \\
h_{i t} & =\omega_{i}+\sum_{k=1}^{p} \alpha_{i k} X_{i, t-k}^{2}+\sum_{k=1}^{q} \beta_{i k} h_{i, t-k},
\end{aligned}
$$

onde os parâmetros $\beta_{i k}, \alpha_{i k}, \omega_{i} \geq 0$ e $X_{i 0}=0, h_{i 0}=0$, e ainda $\sum_{k=1}^{s}\left(\alpha_{i k}+\beta_{i k}\right)<1$ em que $s=\max (p, q)$. Para cada $i$ fixo, as variáveis aleatórias $Y_{i t}$ são i.i.d. com $\mathbb{E}\left[Y_{i t}\right]=0$ e $\operatorname{Var}\left[Y_{i t}\right]=1$, cuja distribuição denotamos por $F_{Y_{i t}}$ e são chamadas de inovações do modelo. O valor $h_{i t} \equiv \operatorname{Var}\left[X_{i t} \mid \mathcal{F}_{t-1}\right]$ onde $\mathcal{F}_{t-1}$ denota o conjunto de informação até o tempo $t$ gerado por $\left\{\boldsymbol{X}_{t-1}, \boldsymbol{X}_{t-2}, \ldots, \boldsymbol{X}_{0}\right\}$. O vetor das inovações $\boldsymbol{Y}_{\boldsymbol{t}} \equiv\left(Y_{1 t}, Y_{2 t}, \ldots, Y_{n t}\right)$ é independente de $\mathcal{F}_{t-1}$.

Quando a cópula associada à distribuição conjunta $F_{\boldsymbol{Y}_{\boldsymbol{t}}}$ é uma D-Vine cópula, a função densidade multivariada de $\boldsymbol{Y}_{\boldsymbol{t}}$ é expressa por,

$$
\begin{aligned}
f_{\mathbf{Y}_{\boldsymbol{t}}}\left(y_{1 t}, y_{2 t}, \ldots, y_{n t}\right)= & \prod_{j=1}^{n-1} \prod_{i=1}^{n-j} c_{i, i+j}\left(F_{i}\left(y_{i t} \mid y_{i+1, t}, \ldots, y_{i+j-1, t}\right), F_{i+j}\left(y_{i+j, t} \mid y_{i+1, t}, \ldots, y_{i+j-1, t}\right)\right) . \\
& \times \prod_{k=1}^{n} f_{k}\left(y_{k, t}\right)
\end{aligned}
$$

Retomamos aqui a mesma formulação (2.13) apresentada na seção 2.1 do Capítulo 2. A função densidade de $\boldsymbol{X}_{\boldsymbol{t}}$ com variância condicional $\boldsymbol{h}_{\boldsymbol{t}}=\left(h_{1 t, \ldots, n t}\right)$, é obtida pela transformação de variável via método do Jacobiano. Sendo a função $\lambda: \mathbb{R}^{n} \rightarrow$ 
$\mathbb{R}^{n}$ com,

$$
\mathbf{X}_{t}=\lambda\left(Y_{1 t}, \ldots, Y_{n t}\right)=\left(Y_{1 t} \sqrt{h_{1 t}}, \ldots, Y_{n t} \sqrt{h_{n t}}\right)
$$

e sua inversa escrita por,

$$
\lambda^{-1}\left(X_{1 t}, \ldots, X_{n t}\right)=\left(\frac{X_{1 t}}{\sqrt{h_{1 t}}}, \ldots, \frac{X_{n t}}{\sqrt{h_{n t}}}\right)
$$

A transformação possuí o jacobiano $J=\prod_{i=1}^{n} \frac{1}{\sqrt{h_{i t}}}$. Logo, a densidade pode ser reescrita da seguinte forma,

$$
\begin{aligned}
& f_{\boldsymbol{X}_{\boldsymbol{t}} \mid \boldsymbol{h}_{\boldsymbol{t}}}\left(\boldsymbol{x}_{\boldsymbol{t}} \mid \boldsymbol{h}_{\boldsymbol{t}}\right) \\
& =\prod_{j=1}^{n-1} \prod_{i=1}^{n-j} c_{i, i+j}\left(F_{i}\left(\frac{x_{i, t}}{\sqrt{h_{i, t}}} \mid \frac{x_{i+1, t}}{\sqrt{h_{i+1, t}}}, . ., \frac{x_{i+j-1, t}}{\sqrt{h_{i+j-1, t}}}\right), F_{i+j}\left(\frac{x_{i+j, t}}{\sqrt{h_{i+j, t}}} \mid \frac{x_{i+1, t}}{\sqrt{h_{i+1, t}}}, . ., \frac{x_{i+j-1, t}}{\sqrt{h_{i+j-1, t}}}\right)\right) \\
& \times \prod_{k=1}^{n}\left|\frac{1}{\sqrt{h_{k, t}}}\right| f_{k}\left(\frac{x_{k, t}}{\sqrt{h_{k, t}}}\right) .
\end{aligned}
$$

Esta formulação é similar ao apresentado por Hofmann e Czado (2011). Com base na estrutura GARCH apresentada e a função densidade multivariada (4.2) seguem as Proposições abaixo.

Proposição 7 Seja $\left(X_{1 t}, X_{2 t}\right)$ o vetor de variáveis aleatórias definido sob o mesmo espaço de probabilidade $(\Omega, \mathcal{F}, \boldsymbol{P})$ com cada variável $X_{i t} i=1,2$ com estrutura $\operatorname{GARCH}(p, q)$, isto é,

$$
\begin{aligned}
X_{i t} & =h_{i t}^{1 / 2} Y_{i t} \\
h_{i t} & =\omega_{i}+\sum_{k=1}^{p} \alpha_{i k} X_{i, t-k}^{2}+\sum_{k=1}^{q} \beta_{i k} h_{i, t-k},
\end{aligned}
$$

onde $X_{1,0}=X_{2,0}=0, h_{1,0}=h_{2,0}=0, \omega_{i k}, \beta_{i k}, \alpha_{i k} \geq 0 \mathrm{com} \sum_{k=1}^{s}\left(\alpha_{i k}+\beta_{i k}\right)<1$ em que $s=\max (p, q)$. Considera-se ainda $\mathbb{E}\left[Y_{1 t}\right]=\mathbb{E}\left[Y_{2 t}\right]=0$ e $\operatorname{Var}\left[Y_{1 t}\right]=$ $\operatorname{Var}\left[Y_{2 t}\right]=1$. Seja também $C\left(u_{1}, u_{2}\right)$ a cópula associada ao vetor de inovações $\left(Y_{1 t}, Y_{2 t}\right)$ com marginais contínuas $F_{Y_{1 t}}$ e $F_{Y_{2 t}}$, então $\forall r \in \mathbb{R}$ quase certamente 
tem-se que a distribuição da soma $X_{1 t}+X_{2 t}$ é dada por,

$$
F_{X_{1 t}+X_{2 t}}\left(r \mid \mathcal{F}_{t-1}\right)=\int_{0}^{1} D_{1} C\left(u_{1}, F_{Y_{2 t}}\left(\frac{r}{\sqrt{h_{1 t}}}-\sqrt{\frac{h_{1 t}}{h_{2 t}}} F_{Y_{1 t}}^{-1}\left(u_{1}\right)\right)\right) d u_{1} .
$$

Prova. Para verificar a proposição acima considere a seguinte distribuição conjunta.

$$
\begin{aligned}
F_{X_{1 t}, X_{1 t}+X_{2 t}}(s, r) & =P\left[X_{1 t} \leq s, X_{1 t}+X_{2 t} \leq r\right] \\
& =\int_{-\infty}^{s} \int_{-\infty}^{r} f_{X_{1 t}, X_{1 t}+X_{2 t}}\left(x_{1 t}, w_{2 t}\right) d x_{1 t} d w_{2 t} \\
& =\int_{-\infty}^{s} \int_{-\infty}^{r} f_{X_{1 t}+X_{2 t} \mid X_{1 t}}\left(w_{2 t} \mid x_{1 t}\right) f_{X_{1 t}}\left(x_{1 t}\right) d x_{1 t} d w_{2 t} \\
& =\int_{-\infty}^{s} F_{X_{1 t}+X_{2 t} \mid X_{1 t}}\left(r \mid x_{1 t}\right) f_{X_{1 t}}\left(x_{1 t}\right) d x_{1 t} \\
& =\int_{-\infty}^{s} P\left[X_{1 t}+X_{2 t} \leq r \mid X_{1 t}=x_{1 t}\right] d F_{X_{1 t}}\left(x_{1 t}\right) .
\end{aligned}
$$

Realizando as substituições $X_{1 t}=h_{1 t}^{1 / 2} Y_{1 t}$ e $X_{2 t}=h_{2 t}^{1 / 2} Y_{2 t}$ tem-se.

$$
\begin{aligned}
F_{X_{1 t}, X_{1 t}+X_{2 t}}(s, r) & =\int_{-\infty}^{s / \sqrt{h_{1 t}}} P\left[\sqrt{h_{1 t}} Y_{1 t}+\sqrt{h_{2 t}} Y_{2 t} \leq r \mid Y_{1 t}=y_{1 t}\right] \frac{1}{\sqrt{h_{1 t}}} f_{Y_{1 t}}\left(y_{1 t}\right) \sqrt{h_{1 t}} d y_{1 t} \\
& =\int_{-\infty}^{s / \sqrt{h_{1 t}}} P\left[Y_{2 t} \leq \frac{r}{\sqrt{h_{2 t}}}-\sqrt{\frac{h_{1 t}}{h_{2 t}}} y_{1 t} \mid Y_{1 t}=y_{1 t}\right] f_{Y_{1 t}}\left(y_{1 t}\right) d y_{1 t} .
\end{aligned}
$$

Pelo Lema 2 é possível expressar a probabilidade condicional em termos da função cópula.

$$
\begin{aligned}
F_{X_{1 t}, X_{1 t}+X_{2 t}}(s, r) & =\int_{-\infty}^{s / \sqrt{h_{1 t}}} D_{1} C\left(F_{Y_{1 t}}\left(y_{1 t}\right), F_{Y_{2 t}}\left(\frac{r}{\sqrt{h_{1 t}}}-\sqrt{\frac{h_{1 t}}{h_{2 t}}} y_{1 t}\right)\right) d F_{Y_{1 t}}\left(y_{1 t}\right) \\
& =\int_{0}^{F_{Y_{1 t}}\left(s / \sqrt{h_{1 t}}\right)} D_{1} C\left(u_{1}, F_{Y_{2 t}}\left(\frac{r}{\sqrt{h_{1 t}}}-\sqrt{\frac{h_{1 t}}{h_{2 t}}} F_{Y_{1 t}}^{-1}\left(u_{1}\right)\right)\right) d u_{1} .
\end{aligned}
$$

No último passo, tomamos a substituição $u_{1}=F_{Y_{1 t}}\left(y_{1 t}\right)$. A distribuição marginal 
de $X_{1 t}+X_{2 t}$ é obtida agora por,

$$
\begin{aligned}
F_{X_{1 t}+X_{2 t}}(r) & =\lim _{s \rightarrow \infty} F_{X_{1 t}, X_{1 t}+X_{2 t}}(s, r) \\
& =\lim _{s \rightarrow \infty} \int_{0}^{F_{Y_{1 t}}\left(s / \sqrt{h_{1 t}}\right)} D_{1} C\left(u_{1}, F_{Y_{2 t}}\left(\frac{r}{\sqrt{h_{1 t}}}-\sqrt{\frac{h_{1 t}}{h_{2 t}}} F_{Y_{1 t}}^{-1}\left(u_{1}\right)\right)\right) d u_{1} .
\end{aligned}
$$

Por fim temos que,

$$
F_{X_{1 t}+X_{2 t}}(r)=\int_{0}^{1} D_{1} C\left(u_{1}, F_{Y_{2 t}}\left(\frac{r}{\sqrt{h_{1 t}}}-\sqrt{\frac{h_{1 t}}{h_{2 t}}} F_{Y_{1 t}}^{-1}\left(u_{1}\right)\right)\right) d u_{1}
$$

Em algumas situações estamos interessados não apenas na distribuição da soma de duas variáveis aleatórias governadas por um processo $\operatorname{GARCH}(p, q)$, mas na distribuição de $X_{1 t}+X_{2 t}+X_{3 t}$. Nesta circunstância, o desenvolvimento é análogo a soma bivariada e é exibida na Proposição abaixo.

Proposição 8 Seja $\left(X_{1 t}, X_{2 t}, X_{3 t}\right)$ um vetor de variáveis aleatórias definidas sob o mesmo espaço de probabilidade $(\Omega, \mathcal{F}, \boldsymbol{P})$ com cada variável $X_{i t} t=0,1, \ldots, T$ e $i=1,2,3$ com estrutura $\operatorname{GARCH}(\mathrm{p}, \mathrm{q})$, isto é,

$$
\begin{aligned}
X_{i t} & =h_{i t}^{1 / 2} Y_{i t}, \\
h_{i t} & =\omega_{i}+\sum_{k=1}^{p} \alpha_{i k} X_{i, t-k}^{2}+\sum_{k=1}^{q} \beta_{i k} h_{i, t-k},
\end{aligned}
$$

onde $X_{i, 0}=0, h_{i, 0}=0, \omega_{i k}, \beta_{i k}, \alpha_{i k} \geq 0 \operatorname{com} \sum_{k=1}^{s}\left(\alpha_{i k}+\beta_{i k}\right)<1$ em que $s=\max (p, q)$. Considera-se ainda $\mathbb{E}\left[Y_{i t}\right]=0$ e $\operatorname{Var}\left[Y_{i t}\right]=1$. Seja também $C\left(u_{1}, u_{2}, u_{3}\right)$ a cópula associada ao vetor de inovações $\left(Y_{1 t}, Y_{2 t}, Y_{3 t}\right)$, com marginais contínuas $F_{Y_{i t}}$, então $\forall r \in \mathbb{R}$ quase certamente tem-se que a distribuição da soma 
$W_{3 t}=X_{1 t}+X_{2 t}+X_{3 t}$ é dada por,

$F_{W_{3 t}}\left(r \mid \mathcal{F}_{t-1}\right)$

$$
=\int_{0}^{1} \int_{0}^{1} D_{12} C\left(u_{1}, u_{2}, F_{Y_{3 t}}\left(\frac{r}{\sqrt{h_{3 t}}}-\sqrt{\frac{h_{1 t}}{h_{3 t}}} F_{Y_{1 t}}^{-1}\left(u_{1}\right)-\sqrt{\frac{h_{2 t}}{h_{3 t}}} F_{Y_{2 t}}^{-1}\left(u_{2}\right)\right)\right) d u_{1} d u_{2} .
$$

Prova. Para verificar a Proposição acima, considere a seguinte distribuição multivariada.

$$
\begin{aligned}
F_{X_{1 t}, X_{2 t}, W_{3 t}}(s, z, r) & =P\left[X_{1 t} \leq s, X_{2 t} \leq z, W_{3 t} \leq r\right] \\
& =\int_{-\infty}^{s} \int_{-\infty}^{z} \int_{-\infty}^{r} f_{X_{1 t}, X_{2 t}, W_{3 t}}\left(x_{1 t}, x_{2 t}, w_{3 t}\right) d x_{1 t} d x_{2 t} d w_{3 t} \\
& =\int_{-\infty}^{s} \int_{-\infty}^{z} \int_{-\infty}^{r} f_{W_{3 t} \mid X_{1 t}, X_{2 t}}\left(w_{3 t} \mid x_{1 t}, x_{2 t}\right) f_{X_{1 t}, X_{2 t}}\left(x_{1 t}, x_{2 t}\right) d x_{1 t} d x_{2 t} d w_{3 t} \\
& =\int_{-\infty}^{s} \int_{-\infty}^{z} P\left[X_{3 t} \leq r-X_{1 t}-X_{2 t} \mid X_{1 t}=x_{1 t}, X_{2 t}=x_{2 t}\right] d F_{X_{1 t}, X_{2 t}}\left(x_{1 t}, x_{2 t}\right) .
\end{aligned}
$$

Realizando as substituições $X_{i t}=h_{i t}^{1 / 2} Y_{i t}$ e pela equação (4.2) temos que,

$$
\begin{aligned}
F_{X_{1 t}, X_{2 t}, W_{3 t}}(s, z, r)= & \int_{-\infty}^{s / \sqrt{h_{1 t}}} \int_{-\infty}^{z / \sqrt{h_{2 t}}} P\left[Y_{3 t} \leq \frac{r-h_{1 t}^{1 / 2} y_{1 t}-h_{2 t}^{1 / 2} y_{2 t}}{h_{3 t}^{1 / 2}} \mid Y_{1 t}=y_{1 t}, Y_{2 t}=y_{2 t}\right] \\
& \times c\left(F_{Y_{1 t}}\left(y_{1 t}\right), F_{Y_{2 t}}\left(y_{2 t}\right)\right) f_{Y_{1 t}}\left(y_{1 t}\right) f_{Y_{2 t}}\left(y_{2 t}\right) \frac{1}{\sqrt{h_{1 t} h_{2 t}}} \sqrt{h_{1 t}} d y_{1 t} \sqrt{h_{2 t}} d y_{2 t} .
\end{aligned}
$$

Pelo Lema 2 é possível escrever a probabilidade condicional em termos da função cópula como expresso em (1.22).

$$
\begin{aligned}
F_{X_{1 t}, X_{2 t}, W_{3 t}}(s, z, r)= & \int_{0}^{F_{Y_{1 t}}\left(s / \sqrt{h_{1 t}}\right)} \int_{0}^{F_{Y_{2 t}}\left(z / \sqrt{h_{2 t}}\right)} \frac{D_{12} C\left(F_{Y_{1 t}}\left(y_{1 t}\right), F_{Y_{2 t}}\left(y_{2 t}\right), F_{Y_{3 t}}\left(\frac{r-h_{1 t}^{1 / 2} y_{1 t}-h_{2 t}^{1 / 2} y_{2 t}}{h_{3 t}^{1 / 2}}\right)\right)}{D_{12} C\left(F_{Y_{1 t}}\left(y_{1 t}\right), F_{Y_{2 t}}\left(y_{2 t}\right)\right)} \\
& \times c\left(F_{Y_{1 t}}\left(y_{1 t}\right), F_{Y_{2 t}}\left(y_{2 t}\right)\right) d F_{Y_{1 t}}\left(y_{1 t}\right) d F_{Y_{2 t}}\left(y_{2 t}\right) .
\end{aligned}
$$

Por fim, realizando as substituições $u_{1}=F_{Y_{1 t}}\left(y_{1 t}\right)$ e $u_{2}=F_{Y_{2 t}}\left(y_{2 t}\right)$ e tomando os 
limites $s \uparrow \infty$ e $z \uparrow \infty$ obtemos a expressão da distribuição marginal de $W_{3 t}$.

$$
F_{W_{3 t}}(r)=\int_{0}^{1} \int_{0}^{1} D_{12} C\left(u_{1}, u_{2}, F_{Y_{3 t}}\left(\frac{r}{\sqrt{h_{3 t}}}-\sqrt{\frac{h_{1 t}}{h_{3 t}}} F_{Y_{1 t}}^{-1}\left(u_{1}\right)-\sqrt{\frac{h_{2 t}}{h_{3 t}}} F_{Y_{2 t}}^{-1}\left(u_{2}\right)\right)\right) d u_{1} d u_{2}
$$

As Proposições 7 e 8 podem ser ainda generalizadas para o caso da soma de $n$ variáveis aleatórias $X_{1 t}+X_{2 t}+\cdots+X_{n t}$ sendo cada uma governada por um processo $\operatorname{GARCH}(\mathrm{p}, \mathrm{q})$.

Proposição 9 Seja $\left(X_{1 t}, \ldots, X_{n t}\right)$ o vetor variáveis aleatórias definidas sob o mesmo espaço de probabilidade $(\Omega, \mathcal{F}, \boldsymbol{P})$ com cada variável $X_{i t} i=1,2, \ldots, n$ e $t=1,2, \ldots, T$, possuindo uma estrutura $\operatorname{GARCH}(\mathrm{p}, \mathrm{q})$,

$$
\begin{aligned}
X_{i t} & =h_{i t}^{1 / 2} Y_{i t} \\
h_{i t} & =\omega_{i}+\sum_{k=1}^{p} \alpha_{i k} X_{i, t-k}^{2}+\sum_{k=1}^{q} \beta_{i k} h_{i, t-k},
\end{aligned}
$$

onde $X_{i, 0}=0, h_{i, 0}=0, \omega_{i k}, \beta_{i k}, \alpha_{i k} \geq 0$ com $\sum_{k=1}^{s}\left(\alpha_{i k}+\beta_{i k}\right)<1$ em que $s=\max (p, q)$. Considera-se ainda $\mathbb{E}\left[Y_{i t}\right]=0$ e $\mathbb{V a r}\left[Y_{i t}\right]=1$. Seja também $C\left(u_{1}, u_{2}, \ldots, u_{n}\right)$ a cópula associada ao vetor de inovações $\left(Y_{1 t}, \ldots, Y_{n t}\right)$ com marginal contínua $F_{Y_{i t}}$, então $\forall r \in \mathbb{R}$ quase certamente tem-se que a distribuição da soma $W_{n t}=X_{1 t}+\cdots+X_{n t}$ é dada por,

$F_{W_{n t}}\left(r \mid \mathcal{F}_{t-1}\right)=\int_{0}^{1} \cdots \int_{0}^{1} D_{1: n-1} C\left(u_{1}, \ldots, F_{Y_{n t}}\left(\frac{r}{h_{n t}^{1 / 2}}-\sum_{i=1}^{n-1} \frac{h_{i t}^{1 / 2}}{h_{n t}^{1 / 2}} F_{Y_{i t}}^{-1}\left(u_{i}\right)\right)\right) d u_{1} \cdots d u_{n-1}$

A prova segue nos mesmos moldes da Proposição 4 do capítulo anterior.

A distribuição da soma de variáveis aleatórias regida por um processo $\mathrm{GARCH}(\mathrm{p}, \mathrm{q})$ pode ser estendida ainda para o caso de uma $A R(b)-\operatorname{GARCH}(p, q)$. A inserção desta estrutura pode ser vantajosa visto que em muitas aplicações de finanças modela-se o retorno de ativos por um processo $\mathrm{AR}(1)-\mathrm{GARCH}(1,1)$. Para tal 
considere a seguinte Proposição abaixo.

Proposição 10 Seja $\left(X_{1 t}, \ldots, X_{n t}\right)$ o vetor de variáveis aleatórias definidas sob o mesmo espaço de probabilidade $(\Omega, \mathcal{F}, \boldsymbol{P})$ com cada variável $X_{i t} i=1,2, \ldots, n$ com estrutura $\operatorname{AR}(1)-\operatorname{GARCH}(1,1)$,

$$
\begin{gathered}
X_{i t}=\mu_{i}+\phi_{i} X_{i, t-1}+h_{i t}^{1 / 2} Y_{i t}, \\
h_{i t}=\omega_{i}+\alpha_{i} X_{i, t-1}^{2}+\beta_{i} h_{i, t-1},
\end{gathered}
$$

onde $X_{i, 0}=0, h_{i, 0}=0, \omega_{i k}, \beta_{i k}, \alpha_{i k} \geq 0 \operatorname{com} \alpha_{i}+\beta_{i}<1$ e $\phi_{i}<1 \forall i=1,2, \ldots, n$. Considera-se ainda $\mathbb{E}\left[Y_{i t}\right]=0$ e $\operatorname{Var}\left[Y_{1 t}\right]=1$. Seja também $C\left(u_{1}, u_{2}, \ldots, u_{n}\right)$ a cópula associada ao vetor de inovações $\left(Y_{1 t}, \ldots, Y_{n t}\right)$ e marginais com função de distribuição contínuas $F_{Y_{i t}}$, então $\forall r \in \mathbb{R}$ quase certamente tem-se que a distribuição da soma $W_{n t}=X_{1 t}+\cdots+X_{n t}$ é dada por,

$$
\begin{aligned}
& F_{W_{n t}}\left(r \mid \mathcal{F}_{t-1}\right) \\
& =\int_{0}^{1} \cdots \int_{0}^{1} D_{1: n-1} C\left(u_{1}, \ldots, F_{Y_{n t}}\left(\frac{r-\sum_{i=1}^{n} \mu_{i}+\phi_{i} x_{i, t-1}}{h_{n t}^{1 / 2}}-\sum_{i=1}^{n-1} \frac{h_{i t}^{1 / 2}}{h_{n t}^{1 / 2}} F_{Y_{i t}}^{-1}\left(u_{i}\right)\right)\right) \\
& \cdot d u_{1} \cdots d u_{n-1} .
\end{aligned}
$$

A prova é imediata a partir da Proposição 9

Por fim, segue a Corolário 8 para o caso da combinação convexa que é análoga ao Corolário 7 apresentado na seção 3.1 do capítulo anterior.

Corolário 8 Seja $\left(X_{1 t}, \ldots, X_{n t}\right)$ o vetor de variáveis aleatórias definidas sob o mesmo espaço de probabilidade $(\Omega, \mathcal{F}, \boldsymbol{P})$ com cada variável $X_{i t} i=1,2, \ldots, n$ com estrutura $\operatorname{AR}(1)-\operatorname{GARCH}(1,1)$,

$$
\begin{aligned}
X_{i t} & =\mu_{i}+\phi_{i} X_{i, t-1}+h_{i t}^{1 / 2} Y_{i t}, \\
h_{i t} & =\omega_{i}+\alpha_{i} X_{i, t-1}^{2}+\beta_{i} h_{i, t-1},
\end{aligned}
$$


onde $X_{i, 0}=0, h_{i, 0}=0, \omega_{i k}, \beta_{i k}, \alpha_{i k} \geq 0 \operatorname{com} \alpha_{i}+\beta_{i}<1$ e $\phi_{i}<1 \forall i=1,2, \ldots, n$. Considera-se ainda $\mathbb{E}\left[Y_{i t}\right]=0$ e $\operatorname{Var}\left[Y_{1 t}\right]=1$. Seja também $C\left(u_{1}, u_{2}, \ldots, u_{n}\right)$ a cópula associada ao vetor de inovações $\left(Y_{1 t}, \ldots, Y_{n t}\right)$ e marginais contínuas $F_{Y_{i t}}$, então $\forall r \in \mathbb{R}$ quase certamente tem-se que a distribuição da combinação convexa $W_{n t}=\sum_{i=1}^{n} \pi_{i} X_{i t}$ é dada por,

$$
\begin{aligned}
& F_{W_{n t}}\left(r \mid \mathcal{F}_{t-1}\right) \\
& =\int_{I^{n-1}} D_{1: n-1} C\left(u_{1}, \ldots, F_{Y_{n t}}\left(\pi_{n}^{-1}\left(\frac{r-\sum_{i=1}^{n} \mu_{i}+\phi_{i} x_{i, t-1}}{h_{n t}^{1 / 2}}-\sum_{i=1}^{n-1} \frac{\pi_{i} h_{i t}^{1 / 2}}{h_{n t}^{1 / 2}} F_{Y_{i t}}^{-1}\left(u_{i}\right)\right)\right)\right) \\
& \cdot d u_{1} \cdots d u_{n-1} .
\end{aligned}
$$





\section{Capítulo 5}

\section{Estimação de Cópulas}

Uma das vantagens da utilização de cópulas é a possibilidade de separação da estrutura de dependência da distribuição marginal. Assim sendo, o método de construção de distribuição multivariada via função cópula desperta o interesse em várias áreas de pesquisa. Em algumas situações as distribuições marginais possuem uma estrutura de regressão, isto é, são condicionadas a covariáveis. Nestes casos, o interesse se concentra na estimação dos parâmetros de regressão. Em outras circunstâncias, o interesse está voltado para a estrutura de dependência, mais especificamente para o valor de $\theta$ da função cópula. Na literatura em finanças, a dependência é empregada em séries temporais como Breymann et al. (2003) e Patton (2001) ou em análise de risco como Embrechts e Juri (2003).

Existem atualmente vários métodos de estimação. Uma técnica usual e direta para estimação de todos os parâmetros é via método de máxima verossimilhança, também conhecida por Full Maximum Likelihood (FML). Uma abordagem alternativa ao FML é o método de estimação em duas etapas conhecida por Two-Step Maximum Likelihood (TSML). Diferentemente do FML em que todos os parâmetros são estimados simultaneamente o TSML estima os parâmetros das marginais e o da função cópula em momentos distintos em razão da segregação da estrutura de dependência e das marginais. A primeira etapa consiste na estimação dos parâmetros das marginais. A segunda etapa utiliza o resultado da primeira para obter 
os parâmetros de dependência da função cópula. O TSML possui ainda algumas variantes dependendo da forma como é feita a primeira etapa (Trivedi e Zimmer, 2005).

Um modelo híbrido sugerido por Song et al. (2005) é a maximização da verossimilhança por partes conhecido como Maximization by Parts (MBP). Outra abordagem possível é por meio dos estimadores dos momentos generalizados (GMM), os quais não serão tratados neste trabalho.

\subsection{Máxima Verossimilhança Exato}

No caso bivariado, ao modelar o par de variáveis aleatórias contínuas $\left(X_{1}, X_{2}\right)$ com uma função cópula qualquer e sendo $F_{1}$ e $F_{2}$ as distribuições marginais acumuladas de $X_{1}$ e $X_{2}$ respectivamente, então a densidade da distribuição conjunta é escrita por,

$$
\begin{aligned}
f\left(x_{1}, x_{2} ; \theta, \Omega\right) & =\frac{\partial^{2}}{\partial x_{1} \partial x_{2}} C\left(F_{1}\left(x_{1}\right), F_{2}\left(x_{2}\right) ; \theta\right) \\
& =\frac{\partial^{2}}{\partial F_{1} \partial F_{2}} C\left(F_{1}\left(x_{1} ; \Omega\right), F_{2}\left(x_{2} ; \Omega\right) ; \theta\right) f_{1}\left(x_{1} ; \Omega\right) f_{2}\left(x_{2} ; \Omega\right) .
\end{aligned}
$$

A função da log-verossimilhança no caso bivariado portanto fica,

$\mathcal{L}_{T}\left(\theta, \Omega \mid \boldsymbol{x}_{1 .}, \boldsymbol{x}_{2 .}\right)=\sum_{t=1}^{T} \ln f_{1}\left(x_{1 t} ; \Omega\right)+\ln f_{2}\left(x_{2 t} ; \Omega\right)+\sum_{t=1}^{T} \ln c\left(F_{1}\left(x_{1 t} ; \Omega\right), F_{2}\left(x_{2 t} ; \Omega\right) ; \theta\right)$

em que $\boldsymbol{x}_{i}$. $=\left(x_{i 1}, x_{i 2}, \ldots, x_{i T}\right)$, é a amostra da série de dados para cada variável $i=1,2$ e $\boldsymbol{x}_{. .}=\left(\boldsymbol{x}_{1 .}, \boldsymbol{x}_{2}\right.$. $)$. $\Omega$ é o conjunto de parâmetros das marginais. Novamente segue a mesma notação $c$ para a densidade cópula.

No caso multivariado onde temos o vetor de variáveis aleatórias contínuas $\left(X_{1}, X_{2}, \ldots, X_{n}\right)$ a densidade conjunta é expressa por,

$$
f\left(x_{1}, x_{2}, \cdots, x_{n} ; \Omega, \theta\right)=c\left(F_{1}\left(x_{1}\right), F_{2}\left(x_{2}\right), \cdots, F_{n}\left(x_{n}\right) ; \theta\right) \prod_{j=1}^{n} f_{i}\left(x_{i} ; \Omega\right) .
$$


Seguindo, obtemos a log-verossimilhança do caso multivariado,

$$
\begin{aligned}
\mathcal{L}_{T}\left(\theta, \Omega \mid \boldsymbol{x}_{1,}, \boldsymbol{x}_{2 .}, \cdots, \boldsymbol{x}_{n} .\right) & =\sum_{t=1}^{T} \ln c\left(F_{1}\left(x_{1 t} ; \Omega\right), F_{2}\left(x_{2 t} ; \Omega\right), \ldots, F_{n}\left(x_{n t} ; \Omega\right) ; \theta\right) \\
& +\sum_{t=1}^{T} \sum_{i=1}^{n} \ln f_{i}\left(x_{i t} ; \Omega\right) .
\end{aligned}
$$

Ainda no caso multivariado $n$-dimensional, porém, com funções cópulas construídas via PCC (Pair Copulas Construction), temos a densidade conjunta expressa por (2.13), a qual é reescrita abaixo,

$$
\begin{aligned}
& f\left(x_{1}, x_{2}, \ldots, x_{n} ; \boldsymbol{\theta}, \Omega\right) \\
& =\prod_{i=1}^{n-1} \prod_{k=1}^{n-j} c_{k, k+i}\left(F\left(x_{k} \mid x_{k+1}, x_{k+2}, \ldots, x_{k+i-1} ; \Omega\right), F\left(x_{k+i} \mid x_{k+1}, x_{k+2}, \ldots, x_{k+i-1} ; \Omega\right)\right) \\
& \times \prod_{i=1}^{n} f_{i}\left(x_{i} ; \Omega\right) .
\end{aligned}
$$

Em virtude da estrutura de dependência ser modelada por um grupo de cópulas, então neste caso temos $d=n(n-1) / 2$ parâmetros de dependência que é dado pelo conjunto $\boldsymbol{\theta}=\left(\theta_{1}, \theta_{2}, \ldots, \theta_{d}\right)$ e o conjunto total de parâmetros é representado por $\boldsymbol{\psi}=(\boldsymbol{\theta}, \Omega)$.

A log-verossimilhança das cópulas construídas via PCC é escrita por,

$$
\begin{aligned}
& \mathcal{L}_{T}\left(\boldsymbol{\theta}, \Omega \mid \boldsymbol{x}_{1 .}, \boldsymbol{x}_{2 .}, \ldots, \boldsymbol{x}_{n .}\right) \\
& =\sum_{t=1}^{T} \sum_{i=1}^{n-1} \sum_{k=1}^{n-i} \ln c_{k, k+i}\left(F\left(x_{k t} \mid x_{k+1, t}, x_{k+2, t}, \ldots, x_{k+i-1, t} ; \Omega\right), F\left(x_{k+i, t} \mid x_{k+1, t}, x_{k+2, t}, \ldots, x_{k+i-1, t} ; \Omega\right) ; \theta_{k, k+i}\right) \\
& +\sum_{t=1}^{T} \sum_{i=1}^{n} \ln f_{i}\left(x_{i t} ; \Omega\right) .
\end{aligned}
$$

Observe que para qualquer um dos casos apresentados (5.2), (5.4) ou (5.5) a 
log-verossimilhança pode ser decomposta em duas parcelas,

$$
\mathcal{L}_{T}(\boldsymbol{\theta}, \Omega)=\mathcal{L}_{1, T}(\Omega)+\mathcal{L}_{2, T}(\boldsymbol{\theta}, \Omega)
$$

A equação (5.6), como função de $\boldsymbol{\theta}$, se resume a segunda parcela, o que pode vir a facilitar o processo de estimação em termos computacionais.

A estimação dos parâmetros no espaço amostral via máxima verossimilhança é obtida por,

$$
\hat{\boldsymbol{\psi}}_{M L}=\underset{\boldsymbol{\psi}}{\arg \max } \mathcal{L}_{T}\left(\boldsymbol{\psi} \mid \boldsymbol{x}_{1}, \boldsymbol{x}_{2}, \ldots, \boldsymbol{x}_{m}\right)
$$

Em termos práticos, $\hat{\boldsymbol{\psi}}_{M L}$ é obtido resolvendo a equação score, $\partial \mathcal{L}_{T} / \partial \boldsymbol{\psi}=\mathbf{0}$. Sob algumas condições de regularidade os estimadores de máxima verossimilhança são consistentes e é assintoticamente normalmente distribuído, i.e.

$$
\sqrt{T}\left(\hat{\boldsymbol{\psi}}_{M L}-\boldsymbol{\psi}\right) \stackrel{d}{\rightarrow} N\left(\mathbf{0}, \mathcal{I}^{-1}\right)
$$

onde $\mathcal{I}$ é a matriz de informação de Fisher. As entradas da matriz são computadas por $(\mathcal{I})_{i, j}=-\mathbb{E}\left[\frac{\partial^{2}}{\partial \theta_{i} \partial \theta_{j}} \log f(X ; \theta)\right]$. Na prática, a matriz de informação de Fisher pode ser estimada pela matriz de informação observada $\mathcal{J}\left(\theta^{*}\right)=$ $-\left.\nabla \nabla^{\top} \mathcal{L}_{T}(\theta)\right|_{\theta=\theta^{*}}$. No caso de má especificação, os erros padrões podem ser obtidos pelo estimador robusto de Huber, também conhecido por estimador Sandwich (Trivedi e Zimmer, 2005).

A estimação via maximização da função log-verossimilhança como (5.4) ou (5.5) pode apresentar algumas dificuldades do ponto de vista computacional, em razão de sua complexidade. Um artifício computacional para superar alguns problemas de otimização de $\mathcal{L}_{T}$ é através da maximização em dois estágios, chamado de Two-Step Maximum Likelihood que será descrito adiante. 


\subsection{Máxima Verossimilhança em Dois Passos}

O método conhecido na literatura por TSML (Two-Step Maximum Likelihood) estima os parâmetros das marginais $\Omega$ em um primeiro momento, já os parâmetros de dependência $\boldsymbol{\theta}$ são obtidos no segundo momento. Dependendo dos parâmetros de interesse e da natureza dos dados há duas estratégicas usuais para tratar a distribuição marginal.

A primeira abordagem não especifica parametricamente as marginais. Nesta circunstância, a distribuição da marginal é obtida pelo estimador de densidade kernel $\hat{f}_{i}\left(x_{i}\right)$ através dos valores de $x_{i t}$ em que $i=1,2, \ldots, m$ e $t=1,2, \ldots, T$. O estimador de densidade kernel possui um parâmetro bandwidth para ajustar a curva da distribuição marginal. Com os valores da amostra obtém-se $\hat{u}_{i t} \equiv \hat{F}_{i}\left(x_{i t}\right)$. Sob um ajuste adequado, os valores $\hat{u}_{i}$. devem apresentar distribuição uniforme. Este resultado já era esperado em virtude do Teorema 13 que se encontra no apêndice. Assim, o conjunto de parâmetros $\boldsymbol{\theta}$ é estimado por,

$$
\hat{\boldsymbol{\theta}}_{T S M L}=\underset{\boldsymbol{\theta}}{\arg \max } \sum_{i=1}^{T} \ln c\left(\hat{u}_{1 t}, \hat{u}_{2 t}, \ldots, \hat{u}_{m t} ; \boldsymbol{\theta}\right) .
$$

Em razão dos valores estimados de $\boldsymbol{\theta}$ serem baseados em dados não diretamente observáveis, os erros padrões de $\hat{\boldsymbol{\theta}}_{T S M L}$ requerem maiores cuidados. A abordagem não paramétrica considera ainda que os valores de $x_{i}$. sejam iid (Trivedi e Zimmer, 2005). No entanto, como sugere Cont (2001), em aplicações com séries temporais financeira não se observa este comportamento. Uma abordagem alternativa é a paramétrica.

Diferentemente da primeira, a segunda abordagem elucida parametricamente as distribuições das marginais. Neste caso, a estimação de $\boldsymbol{\theta}, \Omega$ é feita separadamente a partir da equação (5.6). Primeiramente obtém-se a estimação de $\Omega$ como se segue,

$$
\hat{\Omega}_{T S M L}=\underset{\Omega}{\arg \max } \mathcal{L}_{1, T}(\Omega) .
$$


A estimação de $\boldsymbol{\theta}$ é dada por,

$$
\hat{\boldsymbol{\theta}}_{T S M L}=\underset{\Omega}{\arg \max } \mathcal{L}_{2, T}\left(\boldsymbol{\theta}, \hat{\Omega}_{T S M L}\right) .
$$

Esta abordagem possui a vantagem de ser computacionalmente atrativa quando a dimensão de $\boldsymbol{\theta}$ é grande. Além disso, assim como o FML, o TSML também produz estimativas consistentes de $\boldsymbol{\theta}$ (Trivedi e Zimmer, 2005). Uma forma consistente para se obter os erros padrões é via métodos de bootstrap. O algoritmo pode ser descrito da seguinte maneira:

\section{Algoritmo 5 (Boostrap: Estimação Erro Padrão)}

Passo 1: Obtenha via TSML $\hat{\boldsymbol{\psi}}=(\hat{\boldsymbol{\theta}}, \hat{\Omega})$.

Para cada iteração $k=1,2, \ldots, B$ execute os seguintes passos:

Passo 2 (Amostra Bootstrap): Gere $\boldsymbol{x}_{. .}^{* k}$ através da reamostragem dos dados originais, com reposição.

Passo 3 (Reestimação): Obtenha $\hat{\boldsymbol{\theta}}^{* k}$ e $\hat{\Omega}^{* k}$ com base na amostra $\boldsymbol{x}_{. .}^{* k}$ gerada no passo 2 .

Passo 4 (Erro Padrão): Calcule a matriz de covariância por

$$
B^{-1} \sum_{k=1}^{B}\left(\hat{\boldsymbol{\psi}}^{* k}-\hat{\boldsymbol{\psi}}\right)\left(\hat{\boldsymbol{\psi}}^{* k}-\hat{\boldsymbol{\psi}}\right)^{\prime}
$$

e obtenha a raiz quadrada dos elementos da diagonal principal. 


\section{Capítulo 6}

\section{Ilustrações Numéricas}

Neste capítulo vamos tratar de ilustrações numéricas a partir dos resultados obtidos nos capítulos anteriores. Preliminarmente, investigaremos o caso em que os dados são gerados por simulação. Na sequência, trataremos da análise das medidas de risco por meio de dados reais no mercado financeiro, através da distribuição da soma. Os resultados são obtidos a partir do software R Core Team (2014).

Na seção 6.1, por meio de simulação, investigaremos algumas distribuições de funções de variáveis aleatórias a luz dos resultados das proposições e corolários apresentados nos Capítulos 3 e 4. Previamente determinamos de forma arbitrária alguns conjuntos de parâmetros que definirão o processo gerador de dados. O conjunto contempla os parâmetros das famílias de cópulas e o das funções de distribuição das marginais. Nos dados governados por um processo GARCH serão inclusos ainda os parâmetros da variância condicional para cada distribuição marginal. A partir das realizações das amostras serão estimados os parâmetros via TSML. Posteriormente, obtemos a distribuição de funções de variáveis aleatórias como a soma, combinação convexa e razão. Além disso, são calculadas as medidas de risco $\mathrm{VaR}$ e $\mathrm{ES}^{1}$.

Na seção 6.2 por meio da análise com dados reais, investigamos os principais índices das bolsas de valores do Brasil, Argentina e Chile. A partir das séries

\footnotetext{
${ }^{1}$ A definição do ES encontra-se no Apêndice A
} 
históricas modelamos primeiramente a estrutura de dependência entre estes mercados. Posteriormente, definida a distribuição multivariada dos retornos dos índices, traçamos a distribuição da soma destes retornos. Na sequência, modelamos os retornos considerando a heterocedasticidade dos retornos e calculamos a série do $\operatorname{VaR}_{\alpha, t}\left(W_{n t}\right)$ para uma carteira igualmente ponderada entre os índices.

\subsection{Exemplos por Simulação de Monte Carlo}

O estudo das distribuições de funções de variáveis aleatórias para dados simulados é apresentado em uma sequência crescente de complexidade. Inicialmente, vamos tratar o caso bidimensional para a soma simples, combinação convexa e o caso da soma em que as marginais são governadas por processo GARCH. Em seguida, examinaremos os casos tridimensionais com cópulas construídas via R-Vine cópulas. Outro caso estudado é da distribuição da razão $X_{1} /\left(X_{1}+X_{2}\right)$.

Para determinar a estrutura de dependência entre as duas variáveis aleatórias vamos empregar algumas famílias de cópulas entre elas: arquimedianas Clayton, Frank Joe; de valor extremo Gumbel e as elípticas Normal e t-Student. Um resumo das características dessas cópulas e suas respectivas curvas de níveis pode ser visualizado na Tabela 1.1 e nas Figuras 1.8, 1.10, 1.11, 1.4 e 1.5 nas páginas 52 e 42.

No caso em que as marginais são regidas por uma estrutura GARCH, vamos modelar as inovações $Y_{i t}$ da $i$-ésima marginal no tempo $t$ pela distribuição padrão tStudent com $\nu_{i}$ graus de liberdade. Para obter uma distribuição em que $\mathbb{E}\left[Y_{i t}\right]=0$ e $\operatorname{Var}\left[Y_{i t}\right]=1$ aplicamos uma transformação de escala $Y_{i t}=\left(\frac{\nu_{i}-2}{\nu_{i}}\right)^{1 / 2} Y_{i t}^{\prime}$ em que $Y_{i t}^{\prime} \sim t\left(\nu_{i}\right)$.

As simulação são geradas com o auxílio do pacote CDVine de Brechmann e Schepsmeier (2013), bem como o processo de estimação dos parâmetros dos modelos que será feito via TSML. O método e as características do TSML foram tratados no Capítulo 5. 


\subsubsection{Caso Bivariado}

Em nosso primeiro exemplo vamos tratar do caso bidimensional para descrever a distribuição da soma de $X_{1}+X_{2}$ e algumas de suas características como as medidas de risco. A estrutura de dependência entre $X_{1}$ e $X_{2}$ adotada será a cópula de Clayton. Vamos considerar também que as marginais possuem distribuição t-Student. A função densidade da t-Student é expressa por,

$$
f(y \mid \nu,)=\frac{\Gamma\left(\frac{\nu+1}{2}\right)}{\Gamma\left(\frac{\nu}{2}\right) \sqrt{\pi \nu} \sigma}\left(1+\frac{1}{\nu}\left(\frac{y-\mu}{\sigma}\right)^{2}\right)^{-\frac{\nu+1}{2}},
$$

onde $\nu>0$ representa os graus de liberdade. A função de distribuição acumulada da t-Student e descrita por,

$$
F(y ; \nu, \mu, \sigma)=\frac{1}{2}+\frac{y \Gamma\left(\frac{\nu+1}{2}\right)}{\sqrt{\pi \nu} \Gamma\left(\frac{\nu}{2}\right)}{ }_{2} F_{1}\left(\frac{1}{2}, \frac{\nu+1}{2} ; \frac{3}{2} ;-\frac{y^{2}}{\nu}\right),
$$

em que ${ }_{2} F_{1}$ é a função hipergeométrica ${ }^{2}$. A distribuição t-Student pertence a família de locação-escala, logo pode ser ainda reparametrizada para comportar um parâmetro de locação $\mu$ e um de escala $\sigma$ a partir da transformação $X=\mu+\sigma Y$.

Os parâmetros da distribuição de $X_{1}$ e $X_{2}$ utilizados em nosso exemplo são apresentados na tabela abaixo.

\begin{tabular}{cccc}
\hline \hline Marginal & $\mu$ & $\sigma$ & $\nu$ \\
\hline$X_{1}$ & 2 & 3 & 5 \\
$X_{2}$ & 4 & 5 & 5 \\
\hline \hline
\end{tabular}

Tabela 6.1: Parâmetros distribuição marginal $X_{1}$ e $X_{2}$.

Para cada caso, foram realizadas $K=1.000$ simulações, cada uma sendo gerada com tamanho $N=1.000$ a partir dos valores estabelecidos previamente na Tabela 6.1 e do parâmetro da cópula. A partir de cada simulação são obtidas a estimativa do vetor de parâmetros $\Theta=(\theta, \mu, \sigma, \nu)$. Os valores utilizados para plotar

\footnotetext{
${ }^{2} \mathrm{O}$ desenvolvimento da expressão da acumulada pode ser visto no Apêndice. A
} 
e mensurar as medidas de risco de $X_{1}+X_{2}$ correspondem a média das estimativas $\overline{\hat{\Theta}}=\sum_{i=1}^{K} \hat{\Theta}_{i}$.

Na situação com dependência dada por uma cópula de Clayton serão adotados os parâmetros apresentados na tabela abaixo.

\begin{tabular}{ccccc}
\hline \hline Cópula & $\theta$ & $\tau$ & $\lambda_{L}$ & $\lambda_{U}$ \\
\hline Clayton & 2,3 & 0,53 & 0,73 & 0,0 \\
\hline \hline
\end{tabular}

Tabela 6.2: Parâmetros cópula Clayton

A distribuição conjunta de $Y_{1}=\frac{X_{1}-\mu_{1}}{\sigma_{1}}$ e $Y_{2}=\frac{X_{2}-\mu_{2}}{\sigma_{2}}$ gerada pela cópula de Clayton é exibida no gráfico abaixo.

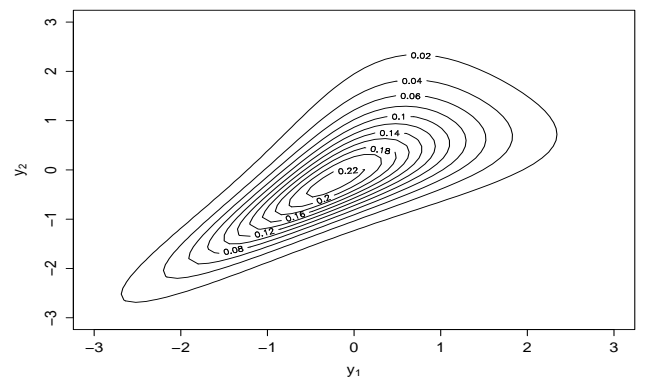

(a) $f_{Y_{1}, Y_{2}}\left(y_{1}, y_{2}\right)$

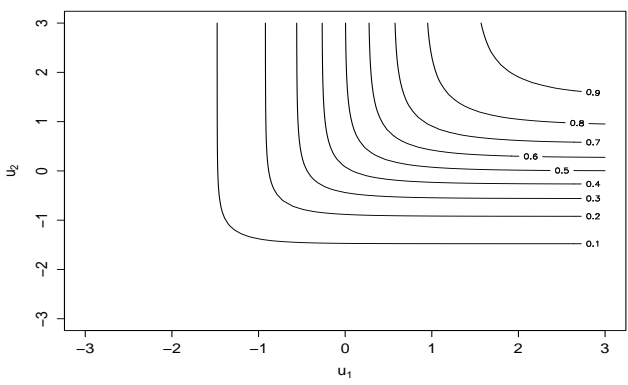

(b) $F_{Y_{1}, Y_{2}}\left(y_{1}, y_{2}\right)$

Figura 6.1: Clayton: curvas de nível com marginais t-Student

Os resultados da estimação média do vetor de parâmetros $\Theta=(\theta, \mu, \sigma, \nu)$, bem como o EQM (Erro Quadráticom Médio) são apresentados na Tabela 6.3. 


\begin{tabular}{|c|c|c|c|c|}
\hline \multicolumn{5}{|c|}{ Marginais } \\
\hline & & $\hat{\mu}$ & $\hat{\sigma}$ & $\hat{\nu}$ \\
\hline \multirow{2}{*}{$X_{1}$} & $\overline{\hat{\Theta}}$ & 2,02 & 3,02 & 5,32 \\
\hline & EQM & $(0,012)$ & $(0,013)$ & $(0,85)$ \\
\hline \multirow{2}{*}{$X_{2}$} & $\overline{\hat{\Theta}}$ & 4,01 & 5,02 & 5,20 \\
\hline & EQM & $(0,033)$ & $(0,033)$ & $(0,805)$ \\
\hline \multicolumn{5}{|c|}{ Cópula } \\
\hline & & $\hat{\theta}$ & $\hat{\tau}-$ Kendall & $\hat{\lambda}_{L}$ \\
\hline \multirow{2}{*}{ Cópula } & $\overline{\hat{\Theta}}$ & 2,29 & 0,53 & 0,73 \\
\hline & EQM & $(0,015)$ & & \\
\hline
\end{tabular}

Tabela 6.3: Estimação parâmetros: cópula Clayton e marginais t-Student.

Através da equação (3.4) foram plotados o gráfico da função densidade $f_{X_{1}+X_{2}}(t)$ e da função de distribuição acumulada $F_{X_{1}+X_{2}}(t)$. As curvas foram obtidas a partir de métodos numéricos.

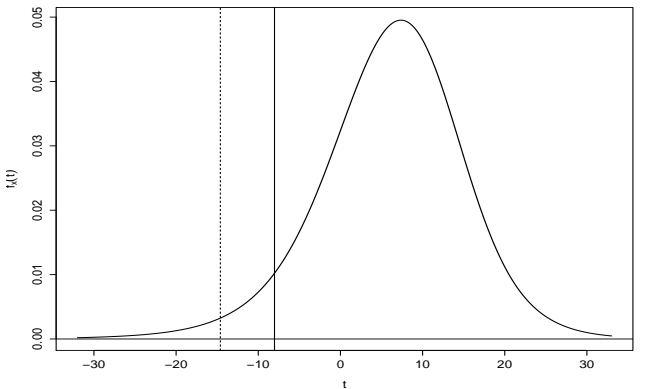

(a) $f_{X_{1}+X_{2}}(t)$

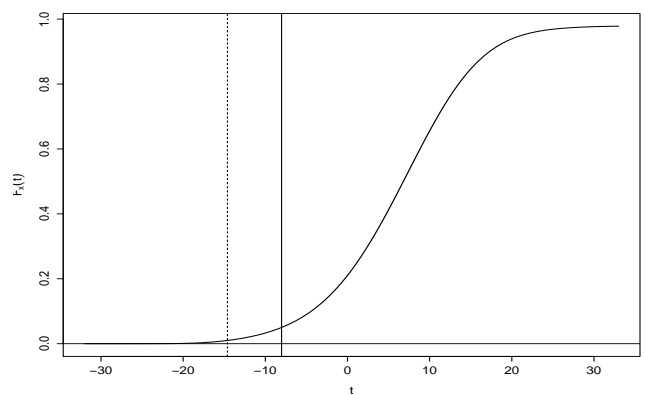

(b) $F_{X_{1}+X_{2}}(t)$

Figura 6.2: Clayton: Value-at-Risk 1\% (tracejada) e 5\% (contínua).

As medidas de risco VaR e ES da distribuição de $X_{1}+X_{2}$ encontram-se na Tabela 6.4 e a variância $\operatorname{Var}\left[X_{1}+X_{2}\right]=66,38$. 


\begin{tabular}{lcccc}
\hline \hline Nível $\alpha$ & $V_{a} R_{\alpha}\left(X_{1}+X_{2}\right)$ & $V a R_{\alpha}\left(X_{1}\right)$ & $V a R_{\alpha}\left(X_{2}\right)$ & $E S_{\alpha}\left(X_{1}+X_{2}\right)$ \\
\hline $5 \%$ & $-8,2$ & $-4,04$ & $-6,82$ & $-16,5$ \\
$1 \%$ & $-14,61$ & $-8,09$ & $-12,82$ & $-39,56$ \\
\hline \hline
\end{tabular}

Tabela 6.4: Medidas de risco de $X_{1}+X_{2}$.

Consideramos agora o caso da distribuição de uma combinação convexa das marginais $w X_{1}+(1-w) X_{2}$. A título ilustrativo vamos tomar $w=0,6$. A função de distribuição para a combinação convexa foi dada pela equação (3.17).

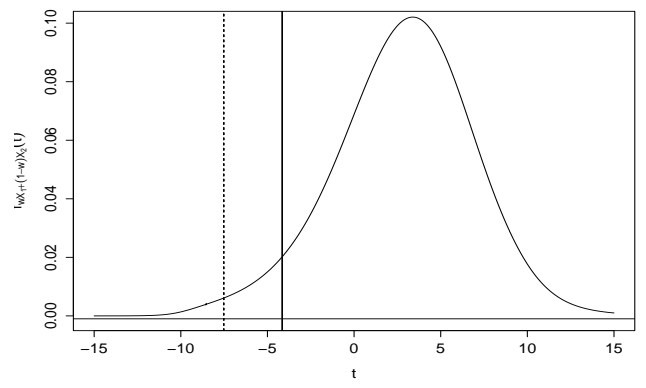

(a) $f_{w X_{1}+(1-w) X_{2}}(t)$

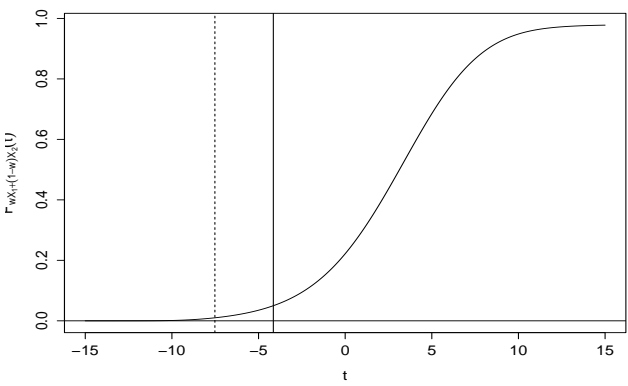

(b) $F_{w X_{1}+(1-w) X_{2}}(t)$

Figura 6.3: Clayton: Value-at-Risk 1\% (tracejada) e 5\% (contínua).

As medidas de risco para a combinação convexa seguem abaixo. Como já era esperado, percebe-se que estas medidas ficaram mais próximas da variável $X_{1}$ visto que ela tem maior peso na combinação.

\begin{tabular}{lcccc}
\hline \hline Nível $\alpha$ & $\operatorname{VaR}_{\alpha}\left(w X_{1}+(1-w) X_{2}\right)$ & $V a R_{\alpha}\left(X_{1}\right)$ & $V a R_{\alpha}\left(X_{2}\right)$ & $E S_{\alpha}\left(w X_{1}+(1-w) X_{2}\right)$ \\
\hline $5 \%$ & $-4,14$ & $-4,04$ & $-6,82$ & $-6,15$ \\
$1 \%$ & $-7,51$ & $-8,09$ & $-12,82$ & $-8,76$ \\
\hline \hline
\end{tabular}

Tabela 6.5: Medidas de risco de $w X_{1}+(1-w) X_{2}$.

No caso em que se deseja otimizar o índice de desempenho Sharpe Ratio ${ }^{3}$ (SR) o valor do peso que maximiza SR é $w^{*}=0,85 \mathrm{com} S R_{w^{*}}=0,2004$. Com o

\footnotetext{
${ }^{3}$ A definição do Índice de Sharpe encontra-se no Apêndice A
} 
peso de $w=0,8$ o índice de Sharpe Ratio é de $S R_{0,8}=0,2005$ próximo ao valor ótimo. A curva abaixo mostra o peso ótimo $w^{*}$.

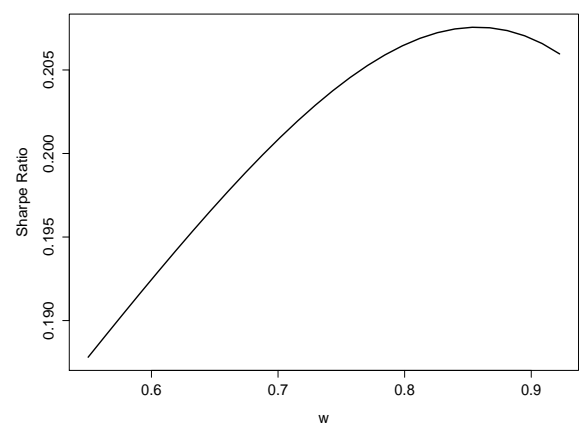

Figura 6.4: Curva Sharpe Ratio.

Estendendo o grau de complexidade, vamos considerar o caso em que as marginais são regidas por um processo $\operatorname{GARCH}(1,1)$. Como mencionado no Capítulo 4, as inovações da $i$-ésima marginal no tempo $t, Y_{i t}$ possuem $\mathbb{E}\left[Y_{i t}\right]=0$ e $\operatorname{Var}\left[Y_{i t}\right]=1$. A tabela abaixo mostra os parâmetros das marginais e da variância condicional que serão utilizadas.

\begin{tabular}{ccccc}
\hline \hline Marginal & $\omega$ & $\alpha$ & $\beta$ & $\nu$ \\
\hline$X_{1}$ & 0,3 & 0,5 & 0,15 & 5,0 \\
$X_{2}$ & 0,3 & 0,5 & 0,15 & 5,0 \\
\hline \hline
\end{tabular}

Tabela 6.6: Parâmetros: distribuição marginal $X_{1}$ e $X_{2}$.

Segue abaixo uma série temporal obtida a partir de uma simulação. 

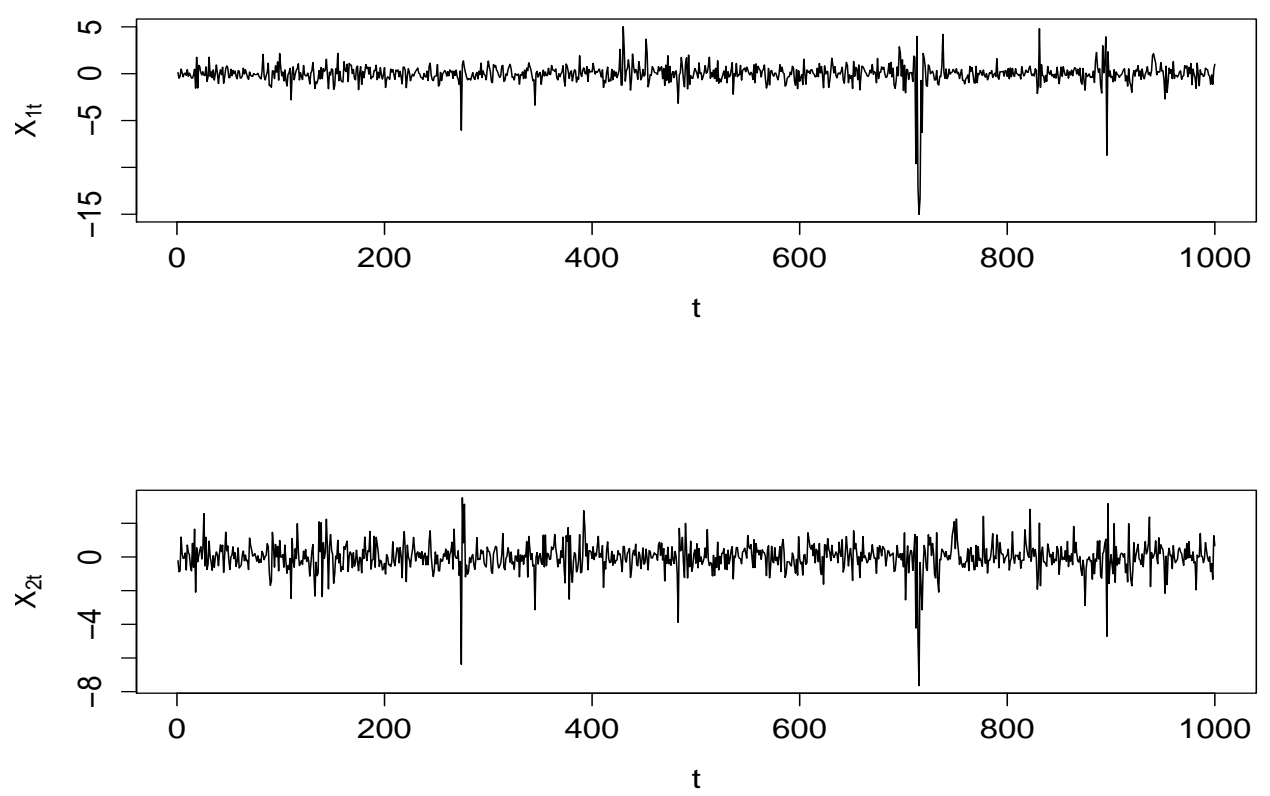

Figura 6.5: Realização do processo $\operatorname{GARCH}(1,1)$ para $X_{1 t}$ e $X_{2 t}$.

Em razão da dependência entre as inovações, perceba que os valores extremos negativos ocorrem praticamente de forma simultânea nos dois gráficos. Esse comportamento é usual nos mercados financeiros, especialmente em períodos de crise, onde há o efeito contágio capitado pelo coeficiente caudal negativo. A Tabela 6.7 mostra as estimativas dos parâmetros das marginais e da cópula. Da mesma maneira, como nos casos anteriores, a estimação foi realizada via máxima verossimilhança em dois estágios (TSML). 


\begin{tabular}{|c|c|c|c|c|c|}
\hline \multicolumn{6}{|c|}{ Marginais } \\
\hline & & $\hat{\omega}$ & $\hat{\alpha}$ & $\hat{\beta}$ & $\hat{\nu}$ \\
\hline \multirow{2}{*}{$X_{1}$} & $\hat{\Theta}$ & 0,302 & 0,494 & 0,144 & 5,38 \\
\hline & EQM & $(0,002)$ & $(0,01)$ & $(0,007)$ & $(0,966)$ \\
\hline \multirow{2}{*}{$X_{2}$} & $\hat{\Theta}$ & 0,308 & 0,501 & 0,142 & 5,25 \\
\hline & EQM & $(0,002)$ & $(0,009)$ & $(0,005)$ & $(0,89)$ \\
\hline \multicolumn{6}{|c|}{ Cópula } \\
\hline & & $\hat{\theta}$ & $\hat{\tau}$ & $\hat{\lambda}_{L}$ & \\
\hline \multirow{2}{*}{ Cópula } & $\hat{\Theta}$ & 2,28 & 0,53 & 0,737 & \\
\hline & EQM & $(0,013)$ & & & \\
\hline
\end{tabular}

Tabela 6.7: Estimação parâmetros: cópula Clayton e GARCH com inovações tStudent.

No caso em que as marginais são governadas por um processo GARCH, o Value-at-Risk $\operatorname{VaR}_{\alpha, t}\left(X_{1 t}+X_{2 t}\right)$ altera em cada tempo para se ajustar a variância condicional. Em função dessas mudanças o $\operatorname{VaR}_{\alpha, t}\left(X_{1 t}+X_{2 t}\right)$ possui um comportamento dinâmico. Os gráficos abaixo exibem a evolução do VaR ao longo do tempo com $\alpha=0,05$ e $\alpha=0,01$, respectivamente. 


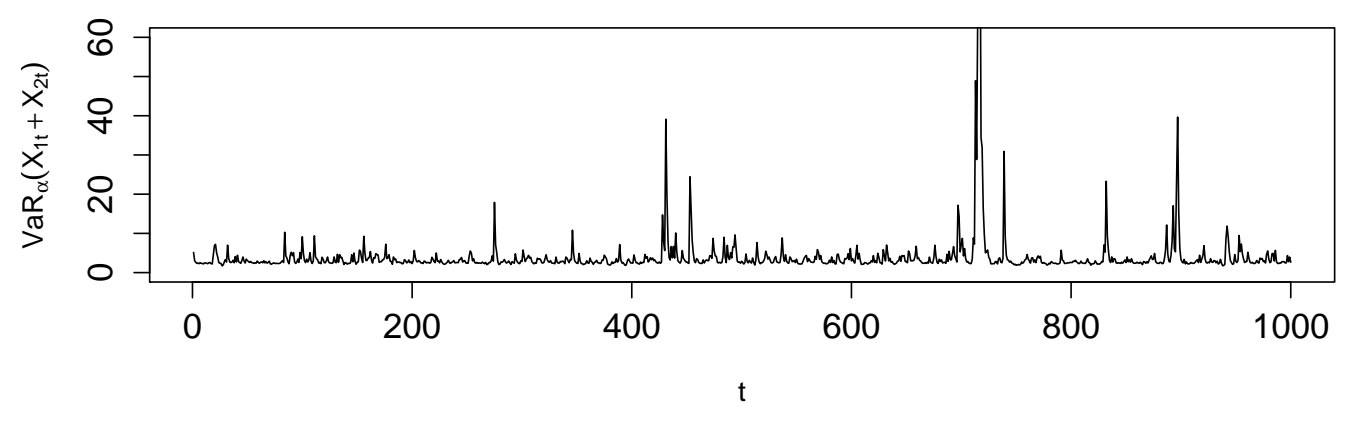

(a) $\alpha=0,05$

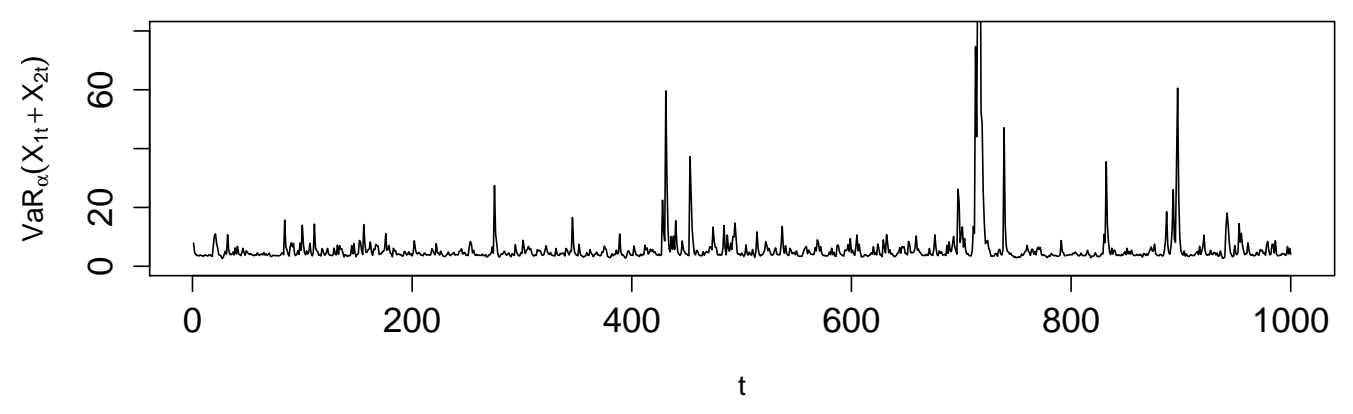

(b) $\alpha=0,01$

Figura 6.6: Value-at-Risk $\operatorname{VaR}_{\alpha}\left(X_{1 t}+X_{2 t}\right)$.

\subsubsection{Caso Multivariado}

No caso multivariado, como discutido em capítulos anteriores, há uma escassez de cópulas $n$-dimensionais na literatura em que o número de dimensões são maiores que 2. Além disso, as cópulas existentes não possuem flexibilidade para acomodar as várias estruturas de dependência entre as variáveis dentro de uma função cópula apenas. Uma alternativa para esse inconveniente é o uso das R-Vines Cópulas para a construção de distribuições com $n \geq 3$. Por conveniência computacional vamos ilustrar o caso em que $n=3$. Os casos em que $n>3$ seguem o mesmo desenvolvimento apresentado a seguir. 
A formulação da distribuição multivariada para o vetor de variáveis aleatórias $\left(X_{1}, X_{2}, X_{3}\right)$ segue da função (2.2). Em nosso exemplo, vamos considerar uma D-Vine e uma C-Vine cópula com configuração de dependência distintas. Suas estruturas são descritas pelos seguintes diagramas,

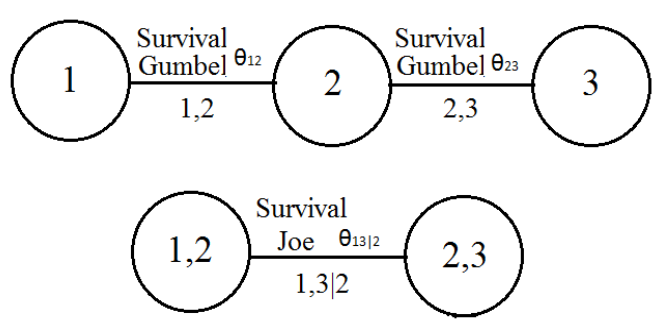

(a) D-Vine

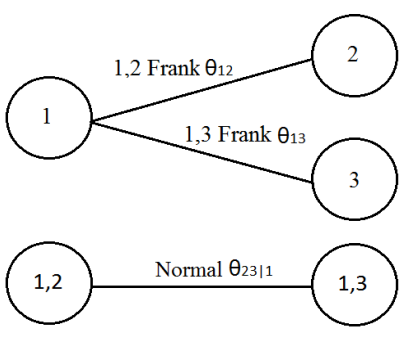

(b) C-Vine

Figura 6.7: Diagramas D-Vine e C-Vine $n=3$.

As cópulas bivariadas selecionadas para a configuração da D-Vine possuem dependência caudal negativa. Este interesse decorre em função do comportamento de dados de séries financeiras. No caso da C-Vine os coeficientes caudais das cópulas bivariadas são todos nulos. A tabela abaixo mostra os valores dos parâmetros para cada cópula que compõe o Diagrama 6.7.

\begin{tabular}{lccccc}
\hline \hline Cópula & Parâmetro & Valor & $\tau$ & $\lambda_{L}$ & $\lambda_{U}$ \\
\hline \multicolumn{5}{c}{ D-Vine } \\
\hline Survival Gumbel & $\theta_{12}$ & 1,2 & 0,16 & 0,22 & 0,0 \\
Survival Gumbel & $\theta_{23}$ & 1,6 & 0,37 & 0,46 & 0,0 \\
Survival Joe & $\theta_{13 \mid 2}$ & 2,0 & 0,35 & 0,59 & 0,0 \\
\hline \multirow{2}{*}{ C-Vine } \\
Frank & $\theta_{12}$ & 2,2 & 0,23 & 0,0 & 0,0 \\
Frank & $\theta_{23}$ & 5,0 & 0,45 & 0,0 & 0,0 \\
Normal & $\theta_{13 \mid 2}$ & 0,4 & 0,40 & 0,0 & 0,0 \\
\hline \hline
\end{tabular}

Tabela 6.8: Parâmetros: D-Vine e C-Vine cópula $n=3$.

As marginais para ambas R-Vines seguem uma distribuição t-Student com 
parâmetros de escala $\sigma$, locação $\mu$ e com $\nu$ graus de liberdade.

\begin{tabular}{cccc}
\hline \hline Marginal & $\mu$ & $\sigma$ & $\nu$ \\
\hline$X_{1}$ & 2,0 & 3,0 & 5,0 \\
$X_{2}$ & 4,0 & 5,0 & 5,0 \\
$X_{3}$ & 2,0 & 5,0 & 6,0 \\
\hline \hline
\end{tabular}

Tabela 6.9: Parâmetros distribuição marginal.

Foram realizadas $K=1.000$ simulações, sendo cada uma delas gerada com tamanho de $N=1.000$ com parâmetros dados nas Tabelas 6.9 e 6.8. As estimativas dos parâmetros, $\Theta=(\theta, \mu, \sigma, \nu)$ correspondem a estimativa média das simulações, isto é, $\bar{\Theta}=\sum_{i=1}^{K} \hat{\Theta}_{i} / K$. Os resultados da estimação, assim como seus erros quadráticos médios (EQM) para a D-Vines e C-Vines, são apresentados nas Tabelas 6.10 e 6.11 , respectivamente.

\begin{tabular}{|c|c|c|c|c|c|c|c|c|c|}
\hline \multicolumn{6}{|c|}{ Marginais } & \multicolumn{4}{|c|}{ D-Vine Cópula } \\
\hline & & $\overline{\hat{\mu}}$ & $\overline{\hat{\sigma}}$ & $\overline{\hat{\nu}}$ & & $\overline{\hat{\theta}}$ & $\overline{\hat{\tau}}$ & $\overline{\hat{\lambda}}_{L}$ & $\overline{\hat{\lambda}}_{U}$ \\
\hline \multirow{2}{*}{$X_{1}$} & $\hat{\Theta}$ & 2,00 & 3,00 & 5,20 & \multirow{2}{*}{$\theta_{12}$} & 1,19 & \multirow{2}{*}{0,36} & \multirow{2}{*}{0,55} & \multirow{2}{*}{0,0} \\
\hline & EQM & $(0,0115)$ & $(0,0136)$ & $(0,8256)$ & & $(0,0013)$ & & & \\
\hline \multirow{2}{*}{$X_{2}$} & $\hat{\Theta}$ & 4,00 & 5,00 & 5,15 & \multirow{2}{*}{$\theta_{23}$} & 1,59 & \multirow{2}{*}{0,54} & \multirow{2}{*}{0,74} & \multirow{2}{*}{0,0} \\
\hline & EQM & $(0,0321)$ & $(0,0373)$ & $(0,811)$ & & $(0,0025)$ & & & \\
\hline \multirow{2}{*}{$X_{3}$} & $\hat{\Theta}$ & 2,00 & 5,00 & 6,32 & \multirow{2}{*}{$\theta_{13 \mid 2}$} & 1,99 & \multirow{2}{*}{0,09} & \multirow{2}{*}{0,13} & \multirow{2}{*}{0,0} \\
\hline & EQM & $(0,0322)$ & $(0,0363)$ & $(1,8801)$ & & $(0,0028)$ & & & \\
\hline
\end{tabular}

Tabela 6.10: Estimação parâmetros: D-Vine cópula e marginais t-Student. 


\begin{tabular}{|c|c|c|c|c|c|c|c|c|c|}
\hline \multicolumn{6}{|c|}{ Marginais } & \multicolumn{4}{|c|}{ C-Vine Cópula } \\
\hline & & $\hat{\mu}$ & $\hat{\sigma}$ & $\hat{\nu}$ & & $\hat{\theta}$ & $\hat{\tau}$ & $\hat{\lambda}_{L}$ & $\hat{\lambda}_{U}$ \\
\hline \multirow{2}{*}{$X_{1}$} & $\hat{\Theta}$ & 1,99 & 3,00 & 5,18 & \multirow{2}{*}{$\theta_{12}$} & 2,17 & \multirow{2}{*}{0,23} & \multirow{2}{*}{0,0} & \multirow{2}{*}{0,0} \\
\hline & EQM & $(0.0109)$ & $(0.0140)$ & $(0.8952)$ & & $(0.0407)$ & & & \\
\hline \multirow{2}{*}{$X_{2}$} & $\hat{\Theta}$ & 4,00 & 5,01 & 5,17 & \multirow{2}{*}{$\theta_{23}$} & 4,96 & \multirow{2}{*}{0,45} & \multirow{2}{*}{0,0} & \multirow{2}{*}{0,0} \\
\hline & EQM & $(0.0316)$ & $(0.0381)$ & $(0.8601)$ & & $(0.0535)$ & & & \\
\hline \multirow{2}{*}{$X_{3}$} & $\hat{\Theta}$ & 1,99 & 4,99 & 6,28 & \multirow{2}{*}{$\theta_{13 \mid 2}$} & 0,39 & \multirow{2}{*}{0,25} & \multirow{2}{*}{0,0} & \multirow{2}{*}{0,0} \\
\hline & EQM & $(0.0295)$ & $(0.0377)$ & $(1.8059)$ & & $(0.0007)$ & & & \\
\hline
\end{tabular}

Tabela 6.11: Estimação parâmetros: C-Vine cópula e marginais t-Student.

De posse destes valores podemos estabelecer a caracterização da cópula DVine trivariada, que possui a seguinte forma,

$$
C\left(u_{1}, u_{2}, u_{3}\right)=\int_{0}^{u_{2}} C_{13 \mid 2}\left(D_{2} C_{12}\left(u_{1}, v_{2} ; \theta_{12}\right), D_{1} C_{23}\left(v_{2}, u_{3} ; \theta_{23}\right) ; \theta_{13 \mid 2}\right) d v_{2}
$$

Pela equação (3.13), a distribuição da soma $W_{3}=X_{1}+X_{2}+X_{3}$, com estrutura de dependência dada pela D-Vine cópula é expressa por,

$$
F_{W_{3}}(t)=\int_{0}^{1} \int_{0}^{1} D_{2,3} \int_{0}^{u_{2}} C_{13 \mid 2}\left(D_{2} C_{12}\left(F_{X_{1}}\left(t-F_{X_{2}}^{-1}\left(v_{2}\right)-F_{X_{3}}^{-1}\left(u_{3}\right)\right), v_{2} ; \theta_{12}\right), D_{1} C_{23}\left(v_{2}, u_{3} ; \theta_{23}\right) ; \theta_{13 \mid 2}\right) d v_{2} d u_{2} d u_{3} \text {. }
$$

No caso da C-Vine cópula trivariada a estrutura é análoga a (6.1), bastando apenas substituir os pares de cópulas e seus parâmetros de acordo com ao Diagrama 6.7b. Os gráficos da distribuição de $W_{3}$ para a D-Vine e C-Vine foram plotados abaixo a partir das equações acima. 


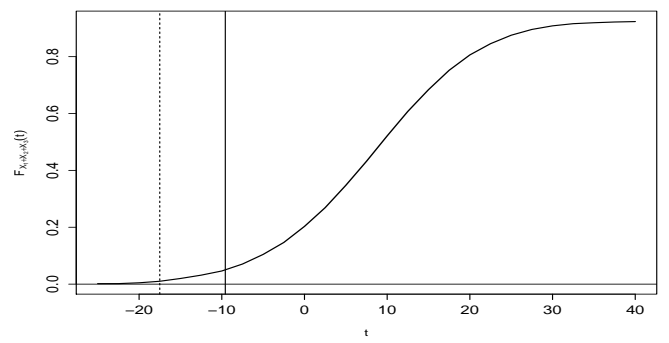

(a) D-Vine

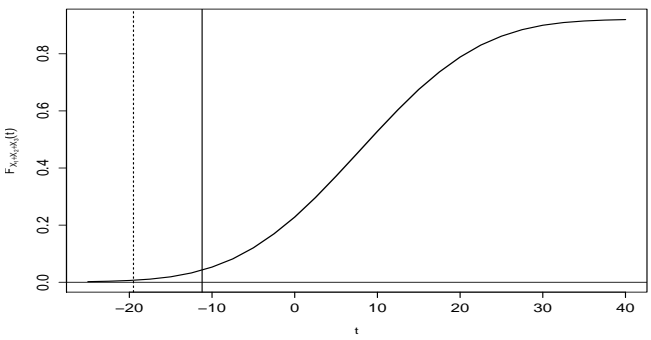

(b) C-Vine

Figura 6.8: D-Vine: $F_{W_{3}}(t)$ Value-at-Risk $1 \%$ (tracejada) e 5\% (contínua).

As medidas de risco VaR e ES da distribuição $W_{3}$ estão dispostas abaixo.

\begin{tabular}{cccc}
\hline \hline \multicolumn{4}{c}{ D-Vine } \\
\hline Nível $\alpha$ & $\operatorname{VaR}_{\alpha}\left(W_{3}\right)$ & $E S_{\alpha}\left(W_{3}\right)$ & $\sum_{i=1}^{3} \operatorname{VaR}_{\alpha}\left(X_{i}\right)$ \\
\hline $5 \%$ & $-9,575$ & $-12,97$ & $-18,19$ \\
$1 \%$ & $-17,5$ & $-25,85$ & $-34,63$ \\
\hline \multicolumn{4}{c}{ C-Vine } \\
\hline $5 \%$ & $-11,2$ & $-15,06$ & $-18,19$ \\
$1 \%$ & $-19,5$ & $-16,50$ & $-34,63$ \\
\hline \hline
\end{tabular}

Tabela 6.12: Medidas de risco de $W_{3}$.

Estendendo o nível de complexidade, vamos inserir uma estrutura GARCH para cada uma das marginais e obter a distribuição da soma. A tabela abaixo mostra os valores dos parâmetros utilizados para modelar a variância condicional.

\begin{tabular}{lcccc}
\hline \hline Marginal & $\omega$ & $\alpha$ & $\beta$ & $\nu$ \\
\hline$X_{1}$ & 0,3 & 0,5 & 0,15 & 5,0 \\
$X_{2}$ & 0,3 & 0,5 & 0,15 & 5,0 \\
$X_{3}$ & 0,3 & 0,5 & 0,15 & 5,0 \\
\hline \hline
\end{tabular}

Tabela 6.13: Parâmetros: distribuição marginal $X_{1}, X_{2}$ e $X_{3}$.

Inicialmente vamos considerar o caso da cópula com estrutura D-Vine dada 
pela Figura 6.7. Um exemplo de uma série gerada a partir da simulação encontra-se abaixo.
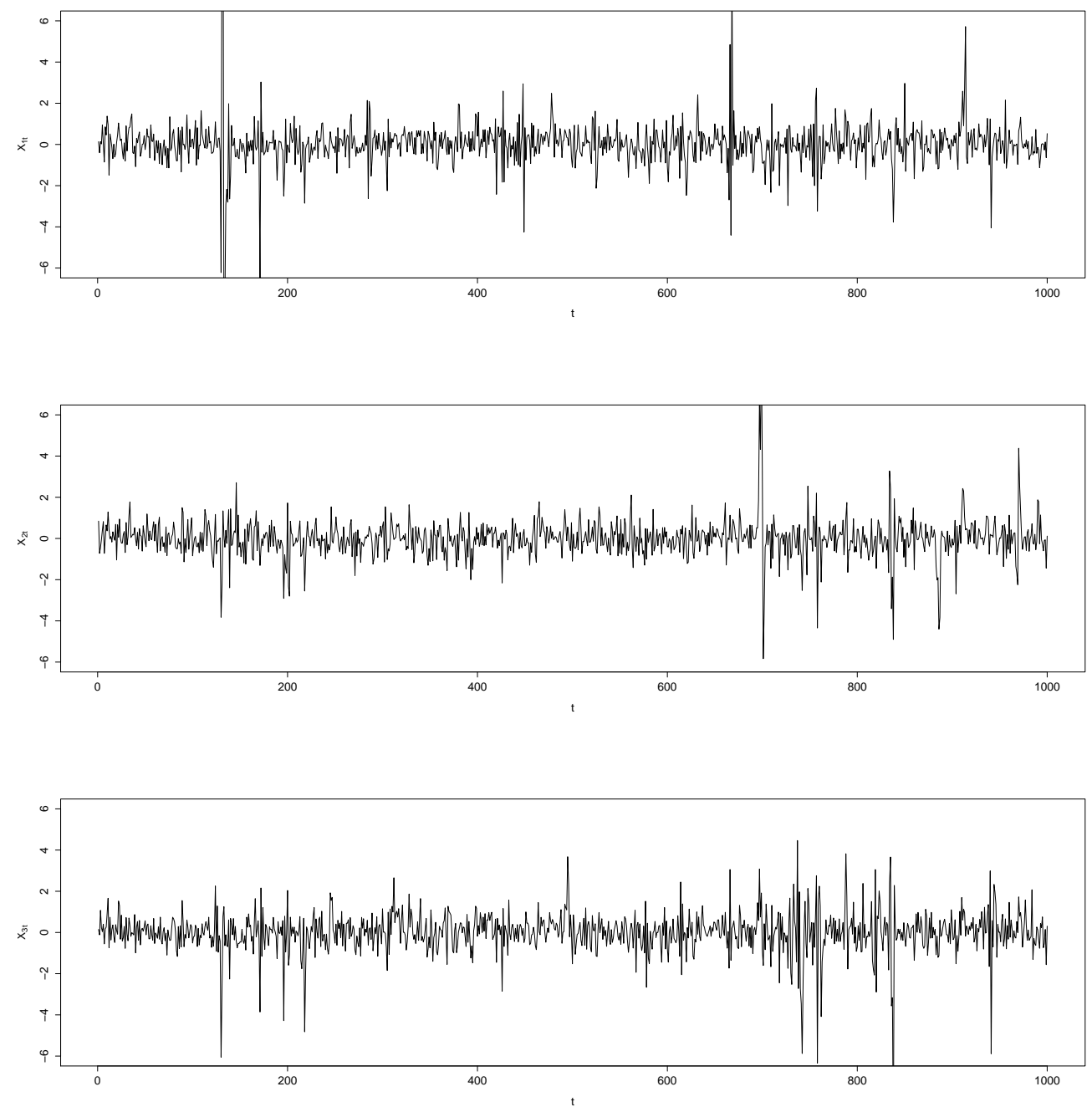

Figura 6.9: Realização do processo $\operatorname{GARCH}(1,1)$ para $X_{1 t}, X_{2 t}$ e $X_{3 t}$.

Novamente, como era já esperado, as quedas acentuadas ocorrem praticamente simultaneamente. Já o comportamento no sentido contrário não é verificado com mesma frequência. Isso se deve ao fato de que as cópulas utilizadas na configuração da D-Vine possuem apenas dependência caudal negativa. 


\begin{tabular}{|c|c|c|c|c|c|}
\hline \multicolumn{6}{|c|}{ Marginais } \\
\hline & & $\hat{\omega}$ & $\hat{\alpha}$ & $\hat{\beta}$ & $\hat{\nu}$ \\
\hline \multirow{2}{*}{$X_{1}$} & $\hat{\Theta}$ & 0,28 & 0,491 & 0,17 & 5,44 \\
\hline & EQM & $(0,002)$ & $(0,008)$ & $(0,005)$ & $(1,32)$ \\
\hline \multirow{2}{*}{$X_{2}$} & $\hat{\Theta}$ & 0,293 & 0,489 & 0,159 & 5,42 \\
\hline & EQM & $(0,002)$ & $(0,008)$ & $(0,004)$ & $(1,15)$ \\
\hline \multirow{2}{*}{$X_{3}$} & $\hat{\Theta}$ & 0,306 & 0,519 & 0,141 & 5,07 \\
\hline & EQM & $(0,002)$ & $(0,009)$ & $(0,004)$ & $(0,76)$ \\
\hline \multicolumn{6}{|c|}{ Cópula } \\
\hline & & $\hat{\theta}$ & $\hat{\tau}$ & $\hat{\lambda}_{L}$ & \\
\hline & $\theta_{12}$ & 1,2 & 0,16 & 0,21 & \\
\hline & EQM & $(0,0009)$ & & & \\
\hline \multirow{4}{*}{ D-Vine Cópula } & $\theta_{23}$ & 1,64 & 0,39 & 0,47 & \\
\hline & EQM & $(0,003)$ & & & \\
\hline & $\theta_{13 \mid 2}$ & 1,81 & 0,31 & 0,53 & \\
\hline & EQM & $(0,04)$ & & & \\
\hline
\end{tabular}

Tabela 6.14: Estimação parâmetros: cópula D-Vine e GARCH com inovações tStudent.

Em detrimento da variância condicional o $\operatorname{VaR}_{\alpha, t}\left(X_{1 t}+X_{2 t}+X_{3 t}\right)$ possui um caráter dinâmico. O gráfico abaixo mostra a realização de uma simulação do $\operatorname{VaR}_{\alpha, t}\left(X_{1 t}+X_{2 t}+X_{3 t}\right)$ com um $\alpha=5 \%$ e $\alpha=1 \%$ para um total de 100 observações. 


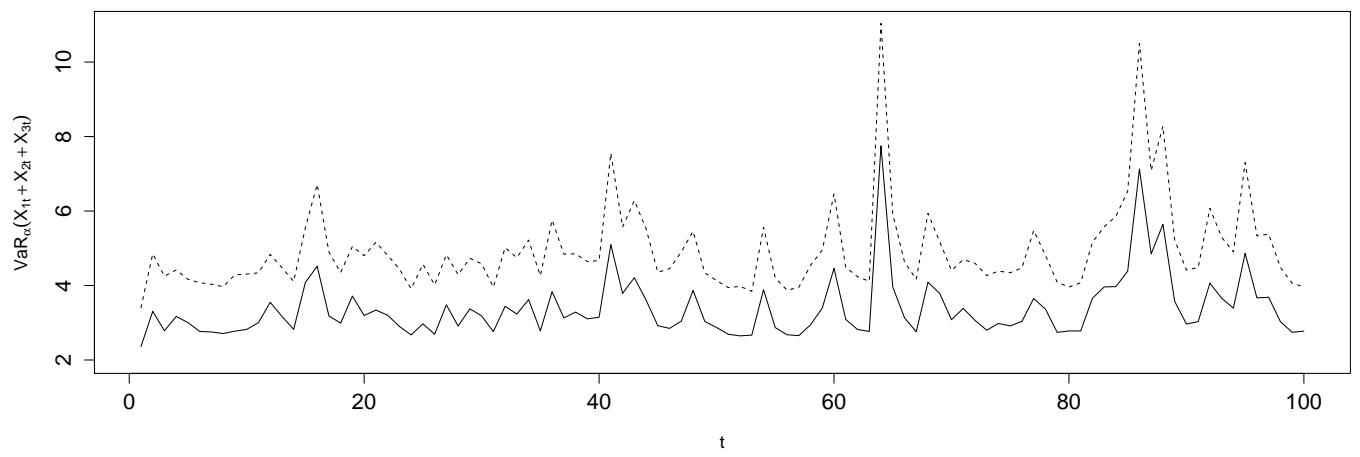

Figura 6.10: Value-at-Risk: $\operatorname{VaR}_{\alpha}\left(X_{1 t}+X_{2 t}+X_{3 t}\right) \alpha=5 \%$ (contínua) $\alpha=1 \%$ (tracejada) 


\subsubsection{Distribuição da Razão}

Uma das distribuições formuladas neste trabalho diz respeito a distribuição da razão $\frac{X_{1}}{X_{1}+X_{2}}$, na qual é expressa pela equação (3.29) apresentada na proposição 6 da seção 3.2. O interesse na distribuição da razão surge em virtude de algumas aplicações, como é o caso da área de hidrologia ou como uma maneira de derivar outras distribuições. Vamos ilustrar o exemplo da razão de variáveis aleatórias com distribuição Gama. A função densidade de uma Gama é definida por,

$$
f(x ; \alpha, \lambda)=\frac{1}{\Gamma(\alpha) \lambda^{\alpha}} x^{\alpha-1} e^{-\frac{x}{\lambda}}
$$

em que $\alpha>0$ é um parâmetro de forma, $\lambda>0$ de escala e $x \in \mathbb{R}^{+}$. A função $\Gamma(\alpha)$ é chamada de gama. Uma variável aleatória $X$ com distribuição gama será denotada por $X \sim \Gamma(\alpha, \lambda)$. Já é bem conhecido que a razão $Y=\frac{X_{1}}{X_{1}+X_{2}}$ em que $X_{1} \sim \Gamma(\alpha, \lambda)$ e $X_{1} \sim \Gamma(\beta, \lambda)$ e sob suposição de independência possui uma distribuição Beta ${ }^{4}$, isto é, $Y \sim B(\alpha, \beta)$. A função densidade Beta é expressa por,

$$
f(x ; \alpha, \beta)=\frac{x^{\alpha-1}(1-x)^{\beta-1}}{B(\alpha, \beta)},
$$

onde a função beta $B(\alpha, \beta)$, também chamada de integral de Euler, é definida por,

$$
B(\alpha, \beta)=\int_{0}^{1} t^{\alpha-1}(1-t)^{\beta-1} \mathrm{~d} t=\frac{\Gamma(\alpha) \Gamma(\beta)}{\Gamma(\alpha+\beta)}
$$

Relaxando a condição de independência entre $X_{1}$ e $X_{2}$ e inserindo a estrutura de dependência via funções cópulas, podemos escrever a função de distribuição acumulada de $Y$. Como as variáveis aleatórias $X_{1}$ e $X_{2}$ estão definidas nos reais positivos, então podemos escrever a expressão da distribuição de $Y$ por,

$$
F_{Y}(t)=1-\int_{0}^{1} D_{1} C\left(u_{1}, F_{X_{2}}\left(\frac{1-t}{t} F_{X_{1}}^{-1}\left(u_{1}\right)\right)\right) d u_{1} .
$$

\footnotetext{
${ }^{4} \mathrm{~A}$ demonstração deste resultado se encontra no Apêndice A
} 
Esta expressão foi apresentada na seção 3.2 como consequência da proposição 6 . Em nosso exemplo vamos considerar arbitrariamente os seguintes parâmetros de $X_{1}$ e $X_{2}$.

\begin{tabular}{ccc}
\hline \hline Marginal & $\alpha$ & $\lambda$ \\
\hline$X_{1}$ & 2,0 & 2,0 \\
$X_{2}$ & 3,0 & 2,0 \\
\hline \hline
\end{tabular}

Tabela 6.15: Parâmetros distribuição Gama.

Para a estrutura de dependência vamos supor a cópula elíptica t-Student. A expressão desta cópula, bem como os gráficos das curvas de nível podem ser vistas em 1.36 e 1.5 nas páginas 40 e 42, respectivamente.

\begin{tabular}{cccccc}
\hline \hline Cópulas & $\rho$ & $\nu$ & $\tau$ & $\lambda_{L}$ & $\lambda_{U}$ \\
\hline Cópula-t I & 0,8 & 3,0 & 0,59 & 0,54 & 0,54 \\
Cópula-t II & $-0,8$ & 3,0 & $-0,59$ & 0,004 & 0,004 \\
Cópula-t III & 0,3 & 5,0 & 0,19 & 0,12 & 0,12 \\
\hline \hline
\end{tabular}

Tabela 6.16: Parâmetros Cópula-t.

Os gráficos abaixo mostram as curvas de nível das distribuições das densidades conjuntas de $X_{1}$ e $X_{2}$ com marginais Gama para cada cópula. 


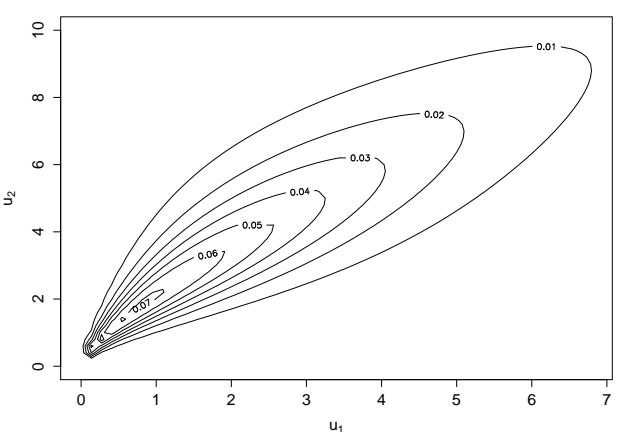

(a) Cópula-t I

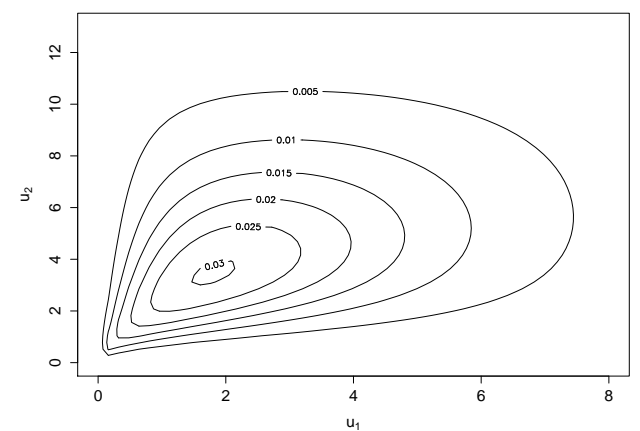

(c) Cópula-t III

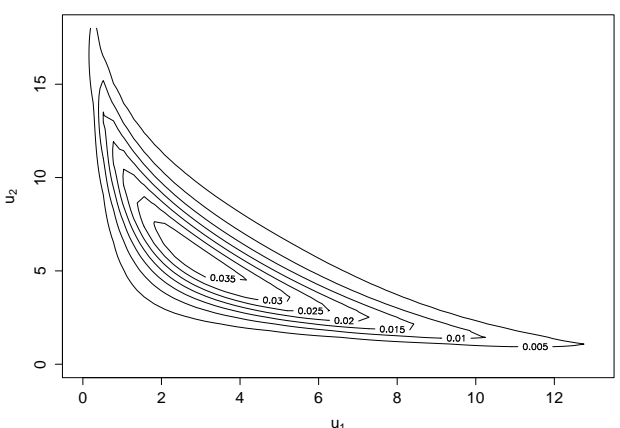

(b) Cópula-t II

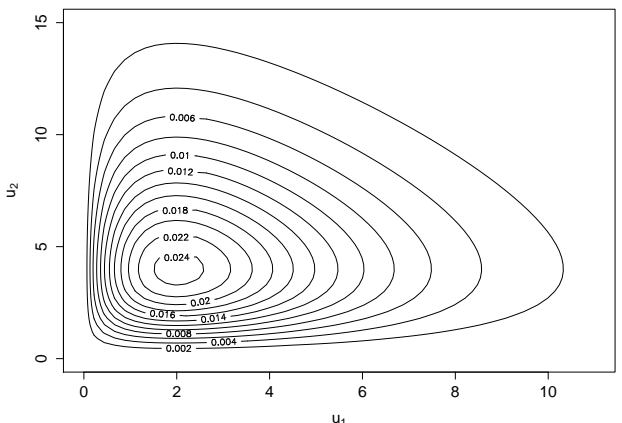

(d) Independente

Figura 6.11: Curvas de níveis das conjuntas $X_{1}$ e $X_{2}$ com marginais Gama

Seguindo os mesmos moldes dos exemplos anteriores, realizamos $K=1.000$ simulações, cada uma contendo $N=1.000$ pares de observação $\left(X_{1}, X_{2}\right)$. Em seguida são calculados os valores médios das $K$ estimativas dos parâmetros. A partir dos valores obtidos na estimação, foram plotados nas Figuras 6.12 os gráficos das funções de distribuição das razões para cada cópula. 


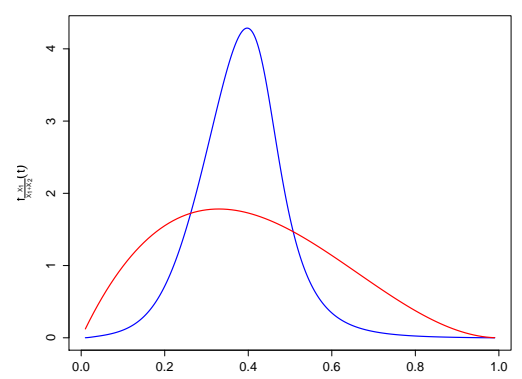

(a) Cópula I

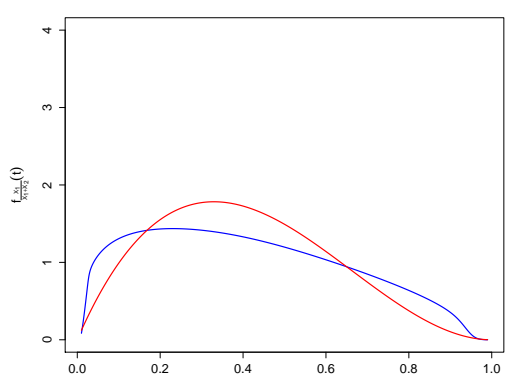

(b) Cópula II

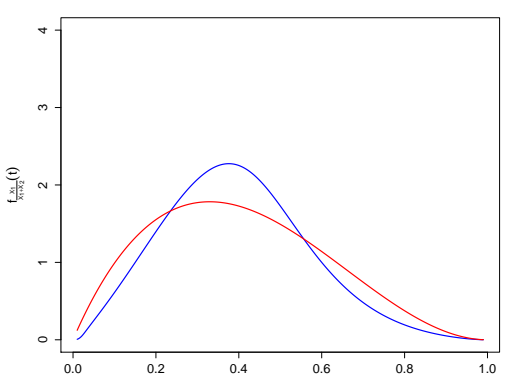

(c) Cópula III

Figura 6.12: Distribuição da razão $Y=\frac{X_{1}}{X_{1}+X_{2}}$ - cópula (azul) e independente (vermelho)

Nos gráficos acima, a curva vermelha mostra a distribuição no caso de independência entre as variáveis $X_{1}$ e $X_{2}$. Como mencionado anteriormente, no caso de independência a razão tem distribuição Beta $Y \sim B(2,3)$. As tabelas abaixo exibem os valores dos resultados das estimativas médias, juntamente com seus respectivos EQM.

\begin{tabular}{|c|c|c|c|c|c|c|c|c|}
\hline \multicolumn{3}{|c|}{ Marginais } & \multicolumn{3}{|c|}{ Marginais } & \multicolumn{3}{|c|}{ Marginais } \\
\hline & $\overline{\hat{\alpha}}$ & $\overline{\hat{\lambda}}$ & & $\overline{\hat{\alpha}}$ & $\overline{\hat{\lambda}}$ & & $\overline{\hat{\alpha}}$ & $\overline{\hat{\lambda}}$ \\
\hline$X_{1}$ & 2,01 & 1,99 & $X_{1}$ & 2,03 & 1,99 & $X_{1}$ & 1,99 & 2,01 \\
\hline EQM & 0,007 & 0,008 & EQM & 0,006 & 0,007 & EQM & 0,006 & 0,008 \\
\hline$X_{2}$ & 3,01 & 1,98 & $X_{2}$ & 2,99 & 2,01 & $X_{2}$ & 3,01 & 1,99 \\
\hline EQM & 0,018 & 0,009 & EQM & 0,018 & 0,009 & EQM & 0,015 & 0,006 \\
\hline
\end{tabular}

\begin{tabular}{|c|c|c|c|c|c|c|c|c|}
\hline \multicolumn{3}{|c|}{ Cópula-t } & \multicolumn{3}{|c|}{ Cópula-t } & \multicolumn{3}{|c|}{ Cópula-t } \\
\hline & $\hat{\rho}$ & $\hat{\nu}$ & & $\hat{\rho}$ & $\hat{\nu}$ & & $\hat{\rho}$ & $\hat{\nu}$ \\
\hline Cópula I & $-0,79$ & 3,07 & Cópula II & 0,79 & 3,08 & Cópula III & 0,29 & 5,25 \\
\hline EQM & 0,0002 & 0,27 & EQM & 0,0002 & 0,2 & EQM & 0,0008 & 1,25 \\
\hline
\end{tabular}

(a) Cópula I

(b) Cópula II

(c) Cópula III

Tabela 6.17: Estimação: Cópula-t e marginais Gama. 


\subsection{Aplicação com Dados Reais}

Nesta seção apresentamos uma aplicação para a distribuição da soma a partir de dados reais em finanças. Os dados são referentes as séries históricas dos principais índices das bolsas de valores das três maiores economias da América Latina: Brasil, Argentina e Chile. No Brasil foi selecionado o índice Ibovespa, na Argentina o Merval e no Chile o índice IPSA ${ }^{5}$. A série é composta pelos valores de fechamento diário dos índices. O período registrado das séries data de 12/05/2003 a 09/10/2015 com um total de $T=2.825$ observações. O gráfico abaixo mostra a evolução histórica dos índices.

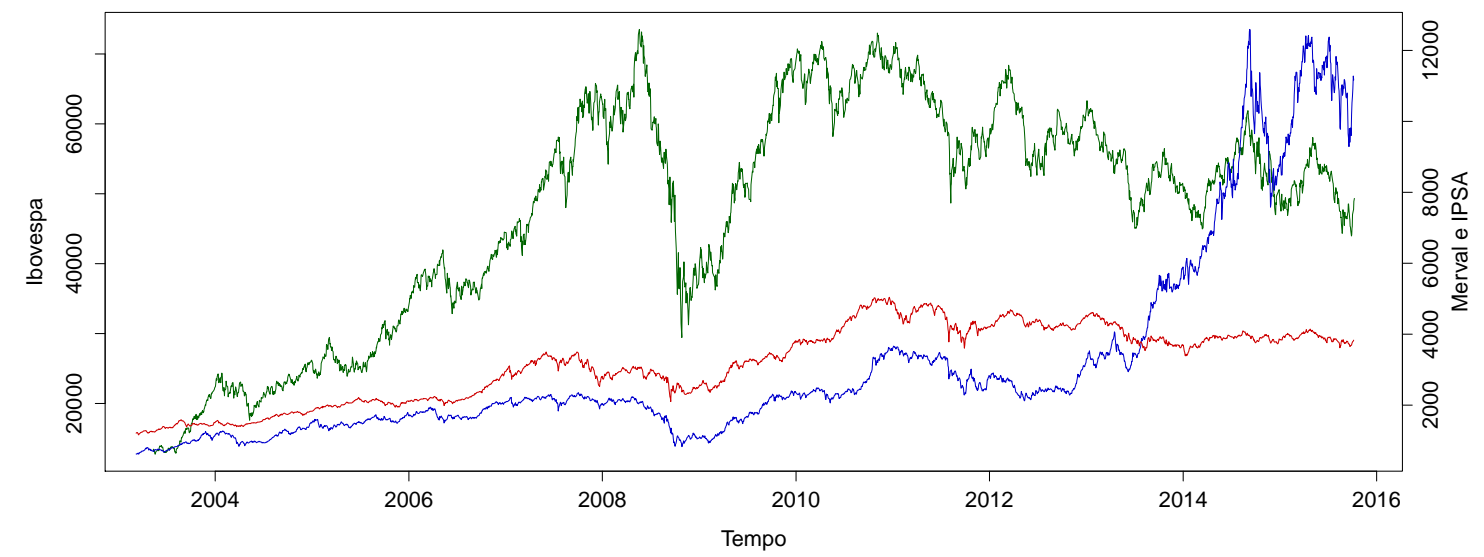

Figura 6.13: Série histórica índices Merval (azul), Ibovespa (verde) e IPSA (vermelho)

O eixo esquerdo do Gráfico 6.13 refere-se a pontuação do Ibovespa e o eixo direito ao IPSA e Merval.

Na sequência computamos os valores dos log retornos os quais são calculados por,

$$
\log _{R_{i}}=\log \left(\frac{p_{i}}{p_{i-1}}\right), i=1,2, \ldots, T
$$

onde $p_{i}$ refere-se ao valor de fechamento diário do índice. A série dos log retornos dos três índices encontra-se na Figura 6.14.

\footnotetext{
${ }^{5}$ Índice de Precio Selectivo de Acciones
} 


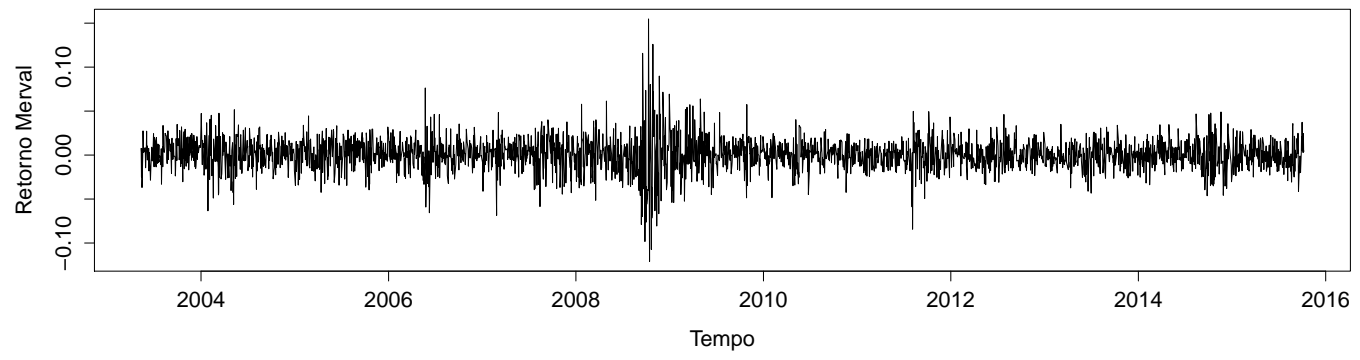

(a) $X_{1}$ :Merval

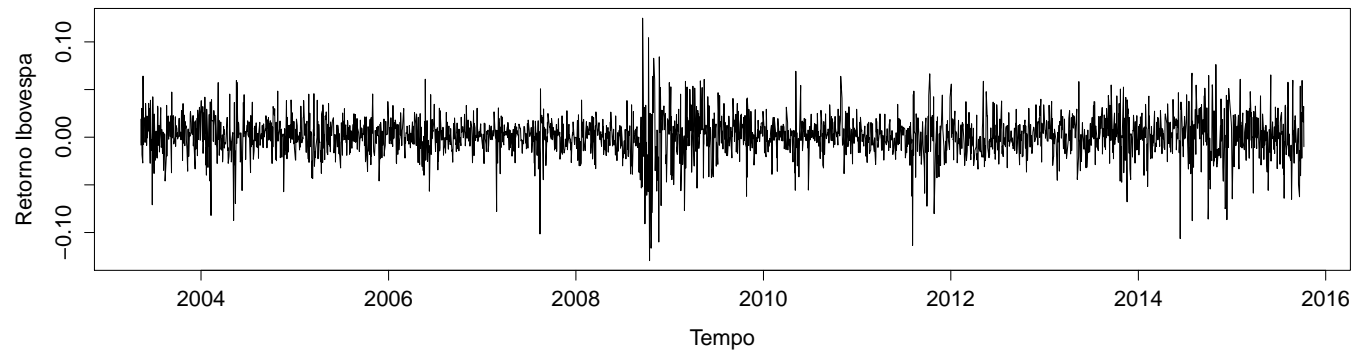

(b) $X_{2}$ :Ibovespa

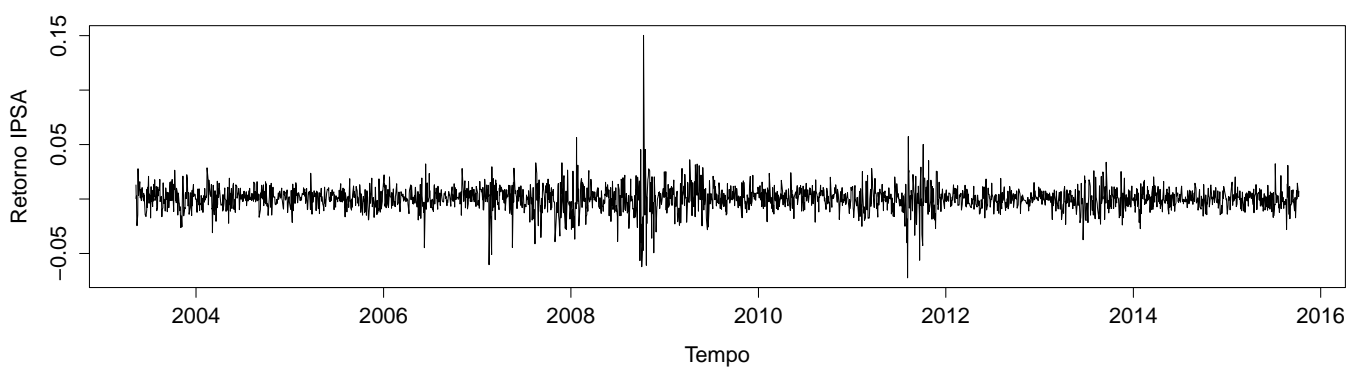

(c) $X_{3}:$ IPSA

Figura 6.14: Série retornos dos índices.

O gráfico apresenta uma simetria nos períodos de maiores volatilidades. Visualmente é possível observar esse comportamento no período de 2009 e no final de 2011. Essa característica é análoga ao que ocorreu na seção anterior, onde estamos interessados na distribuição da soma de variáveis aleatórias em que as marginais eram regidas por um processo $\operatorname{GARCH}(1,1)$, sendo as inovações interdependentes. Denotaremos por $X_{1}, X_{2}$ e $X_{3}$ os log's retornos diários dos índices Merval, 
Ibovespa e IPSA, respectivamente.

Os testes de Dickey-Fuller Aumentado apontaram para a estacionaridade da série dos retornos dos índices. No que tange a autocorrelação dos retornos, segundo o teste de Ljung-Box, apenas índice Merval não apresentou autocorrelação. Os teste de estacionaridade ADF, os testes de Ljung-Box e os gráficos das funções autocorrelação encontram-se no Apêndice B.

No primeiro momento vamos analisar o caso em que os retornos dos três índices possuem distribuição conjunta com estrutura de dependência dada por uma D-Vine Cópula. A série temporal dos retornos é inicialmente considerada homocedástica. Em seguida calculamos as medidas de risco $V a R$ e ES de uma carteira composta por esses índices. As medidas de risco são calculadas a partir da função de distribuição da variável aleatória $W_{3}=X_{1}+X_{2}+X_{3}$ descrita na Proposição 3. O diagrama da configuração da D-Vine cópula empregada no estudo é apresentado na Figura 6.15.

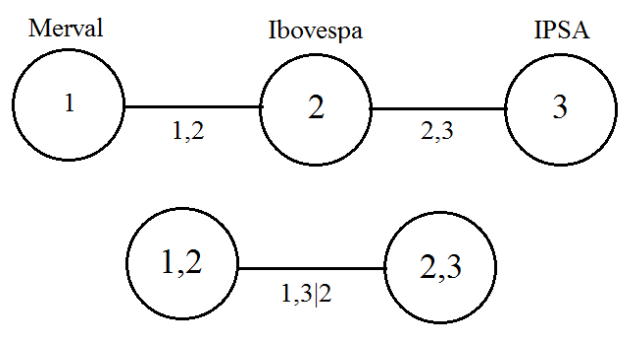

Figura 6.15: Diagrama D-Vine cópula

No segundo momento, vamos considerar a mesma estrutura de dependência, porém assumindo a heterocedasticidade dos dados de retorno. As marginais da distribuição conjunta dos retornos são modeladas um processo $\operatorname{GARCH}(1,1)$ nos mesmos moldes descritos em (4.1). Na sequência, computamos a série histórica das medidas de risco $V a R$ e $E S$ a partir da distribuição da soma das marginais obtidas no Capítulo 4.

A estimação dos parâmetros da distribuição conjunta de $X_{i} i=1,2,3$ é feita novamente via TSML. Para cada uma das marginais estimamos duas distribuições, 
normal e t-Student. O Gráfico 6.17 exibe os ajustes das estimativas das curvas juntamente com o estimador kernel da densidade dos retornos.

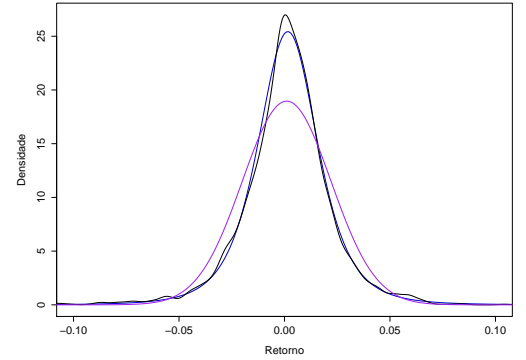

(a) Merval

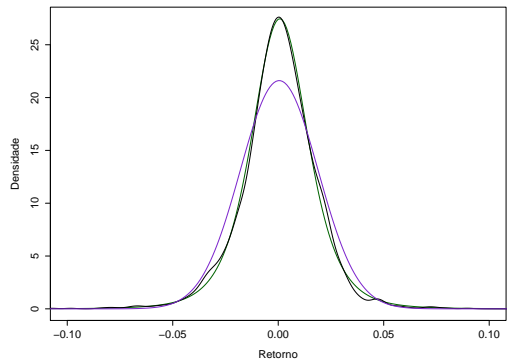

(b) Ibovespa

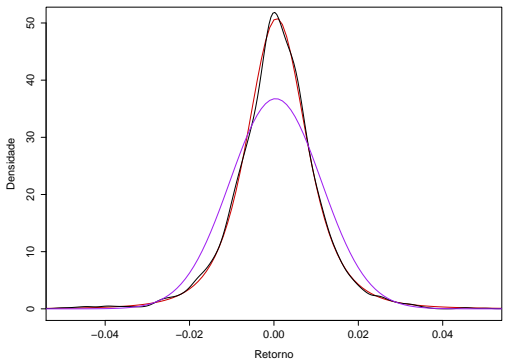

(c) IPSA

Figura 6.16: Densidade t-Student, Normal (roxo) e estimador Kernel (preto).

Visualmente é possível notar que a distribuição t-Student se ajustou melhor aos dados. Essa noção é corroborada pelas medidas de qualidade de ajuste AIC e BIC.

\begin{tabular}{ccccc}
\hline \hline & \multicolumn{2}{c}{ Normal } & \multicolumn{2}{c}{ t-Student } \\
Marginal & AIC & BIC & AIC & BIC \\
\hline$X_{1}$ & -13791.05 & -13779.15 & -14214.28 & -14196.44 \\
$X_{2}$ & -14529.9 & -14518.01 & -14892.89 & -14875.05 \\
$X_{3}$ & -17531.55 & -17519.65 & -18149.14 & -18131.3 \\
\hline \hline
\end{tabular}

Tabela 6.18: AIC e BIC marginais

\begin{tabular}{|c|c|c|c|c|c|c|}
\hline Marginal & $\hat{\mu}$ & $\hat{\sigma}$ & $\hat{\nu}$ & e.p $(\hat{\mu})$ & $e . p(\hat{\sigma})$ & e.p $(\hat{\nu})$ \\
\hline$X_{1}$ & 0.00158 & 0.01469 & 3.8016 & 0.0003 & 0.0004 & 0.92 \\
\hline$X_{2}$ & 0.00074 & 0.0137 & 4.5129 & 0.0003 & 0.0006 & 0.99 \\
\hline$X_{3}$ & 0.00065 & 0.00733 & 3.60694 & 0.0001 & 0.0002 & 0.78 \\
\hline
\end{tabular}

Tabela 6.19: Parâmetros estimado distribuição t-Student

Os valores dos erros padrões e.p $(\hat{\mu})$, e.p $(\hat{\sigma})$, e.p $(\hat{\nu})$ foram obtidos via bootstraps com $B=1000$ amostra. Em virtude dos dados apresentarem autocorrelação 
e heterocedasticidade empregamos neste caso o bootstrap em blocos não sobrepostos. Para dimensionar os blocos adotamos o critério sugerido por Hall et al. (1995). A partir da definição dos blocos o processo de reamostragem realizado de acordo com o Algoritmo 5 na seção 5.2.

Por intermédio dos parâmetros estimados obtém-se as transformações dos dados $\hat{u}_{i j}=F_{X_{i}}\left(x_{i j} \mid \hat{\mu}, \hat{\sigma}, \hat{\nu}\right)$, em que $i=1,2,3$ e $j=1,2, \ldots, T$. Se os dados estiverem bem ajustados, pelo Teorema 13 os valores de $U_{i} \sim U(0,1)$. Pelo histograma abaixo os valores se ajustam bem próximos a uma distribuição uniforme.

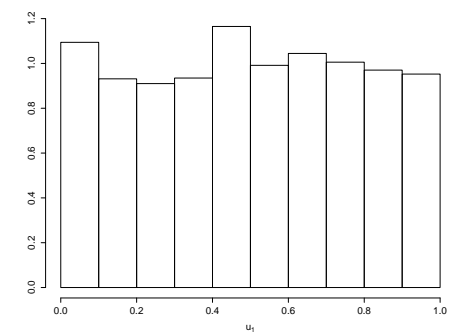

(a) Merval

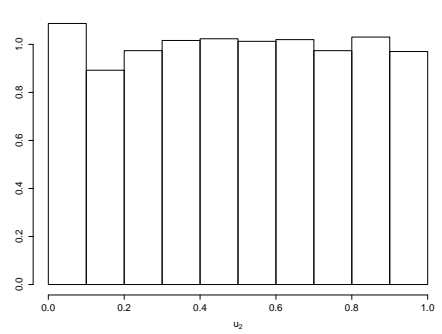

(b) Ibovespa

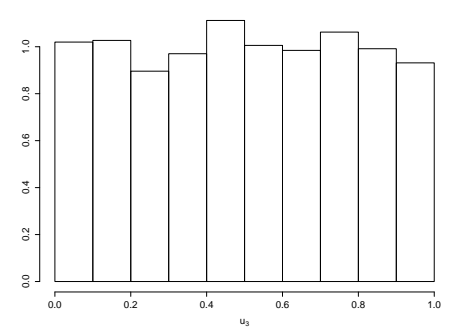

(c) IPSA

Figura 6.17: Histograma $\hat{u}_{i}=F_{X_{i}}\left(x_{i}: \hat{\mu}, \hat{\sigma}, \hat{\nu}\right)$

A partir dos valores $\hat{u}_{i j}$ foi plotado o gráfico de dispersão e a cópula empírica.

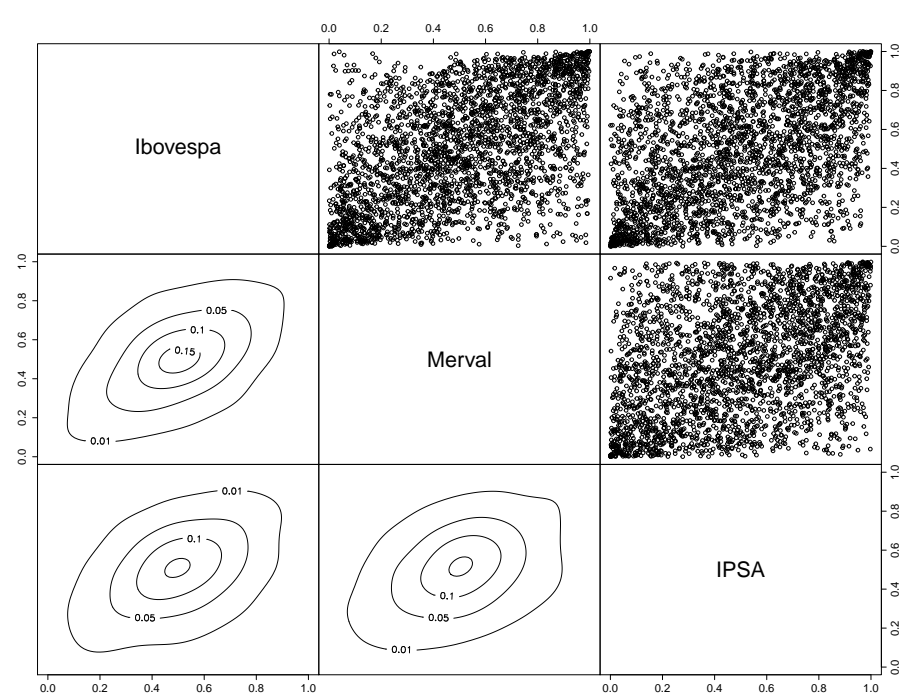

Figura 6.18: Matriz de dispersão e cópula empírica dos retornos dos índices 
Para testar a independência entre os retornos, empregamos o teste proposto por Genest e Favre (2007), baseado no coeficiente de correlação $\tau$ de Kendall. O teste é assintoticamente normalmente distribuído e é calculado por,

$$
K=\sqrt{\frac{9 T(T-1)}{2(2 T+5)}}|\hat{\tau}|
$$

sendo $\hat{\tau}$ o valor empírico do $\tau$-Kendall. Os testes para todos os pares de variáveis não apontaram para independência. Para selecionar as cópulas $C_{12}\left(u_{1}, u_{2}\right)$, $C_{23}\left(u_{2}, u_{3}\right)$ e $C_{13 \mid 2}\left(u_{1 \mid 2}, u_{3 \mid 2}\right)$ descritas no Diagrama 6.15, vamos utilizar o método de seleção proposto por Belgorodski (2010). O método compara para cada cópula $C_{a}$ com as demais cópulas propostas por um teste. Cada vez que a cópula $C_{a}$ é preferida em detrimento de outra, soma-se +1 ponto, caso contrário subtrai-se -1. O teste de comparação aplicado par a par é o de Vuong (1989). Neste teste compara-se duas cópulas $C_{1}$ e $C_{2}$ através da densidade da cópula e dos parâmetros estimados. A partir dos valores $\kappa_{i}=\log \left(\frac{c_{1}\left(u_{i 1}, u_{i 2} ; \hat{\theta}_{1}\right)}{c_{2}\left(u_{i 1}, u_{i 2} ; \hat{\theta}_{2}\right)}\right)$ para $i=1,2, \ldots, T$ computase a estatística,

$$
\eta=T^{-1} \frac{\sum_{i=1}^{T} \kappa_{i}}{\left(\sum_{i=1}^{T}\left(\kappa_{i}-\bar{\kappa}\right)^{2} / T\right)^{\frac{1}{2}}} .
$$

A estatística $\eta$ é assintoticamente normalmente distribuída. A cópula $C_{1}$ é preferida em relação a $C_{2}$, a um nível de $\alpha$, se $\eta>\Phi^{-1}\left(1-\frac{\alpha}{2}\right)$. Maiores detalhes deste e outros métodos podem ser vistos em Brechmann e Schepsmeier (2013). A tabela abaixo mostra os resultados do método de seleção.

\begin{tabular}{ccccccc}
\hline \hline Cópula & Gaussiana & t-Student & Clayton & Gumbel & Frank & Joe \\
\hline$C_{12}\left(u_{1}, u_{2}\right)$ & 2 & 5 & -1 & 1 & -2 & -5 \\
$C_{23}\left(u_{2}, u_{3}\right)$ & 3 & 5 & -1 & 0 & -2 & -5 \\
$C_{13 \mid 2}\left(u_{1 \mid 2}, u_{3 \mid 2}\right)$ & 0 & 1 & -5 & 5 & -1 & 0 \\
\hline \hline
\end{tabular}

Tabela 6.20: Seleção cópulas baseada no teste de Vuong

Baseado no método de seleção, as cópulas que apresentaram melhores ajustes para as cópulas $C_{12}\left(u_{1}, u_{2}\right), C_{23}\left(u_{2}, u_{3}\right)$ e $C_{13 \mid 2}\left(u_{1 \mid 2}, u_{3 \mid 2}\right)$ foram t-Student, t-Student 
e Gumbel, respectivamente. Uma análise visual do ajuste pode ser vista a partir da função $\lambda$ proposto por Genest et al. (1993). A função é expressa por,

$$
\lambda(v, \theta)=v-K(v, \theta)
$$

em que $K(v, \theta)=P\left(C\left(U_{1}, U_{2} \mid \hat{\theta}\right) \leq v\right), \hat{\theta}$ e $v \in[0,1]$. Para as cópulas arquimedianas a função $K$ possui expressão analítica, já para as cópulas elípticas Gaussiana e t-Student não têm, e é estimado por simulação. Os limites inferior $\tau=0$ e superior $\tau=1(\lambda=0)$ correspondem as linhas inferiores e superiores no gráfico. O gráfico e a função $\lambda$ estão implementados no pacote Brechmann e Schepsmeier (2013).

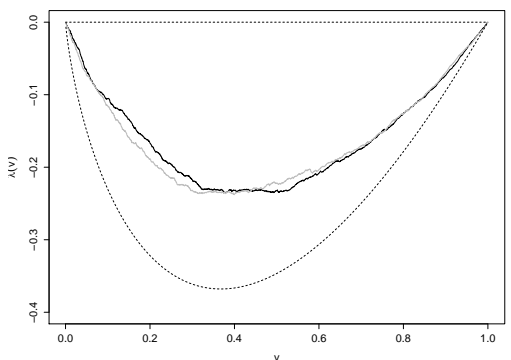

(a) Merval x Ibovespa

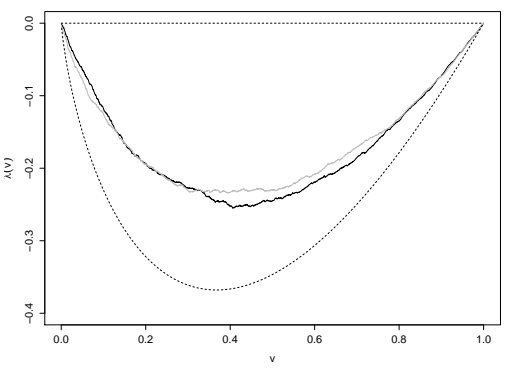

(b) Ibovespa x IPSA

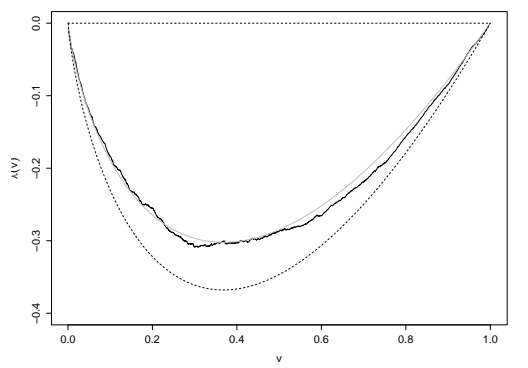

(c) Merval x IPSA | Ibovespa

Figura 6.19: Função $\lambda$ : teórico (cinza) empírico (preto)

As estimações conjuntas dos parâmetros por máxima verossimilhança das cópulas com melhores ajustes são apresentadas na tabela abaixo.

\begin{tabular}{cccccccc}
\hline \hline Cópula & $\hat{\theta}$ & $\hat{\nu}$ & $\mathrm{e} . \mathrm{p}(\hat{\theta})$ & $\mathrm{e} . \mathrm{p}(\hat{\nu})$ & $\hat{\tau}$ & $\lambda_{U}$ & $\lambda_{L}$ \\
\hline$C_{12}\left(u_{1}, u_{2}\right)$ & 0.56 & 4.75 & 0.015 & 0.81 & 0.37 & 0.25 & 0.25 \\
$C_{23}\left(u_{2}, u_{3}\right)$ & 0.51 & 6.88 & 0.015 & 1.88 & 0.34 & 0.15 & 0.15 \\
$C_{13 \mid 2}\left(u_{1 \mid 2}, u_{3 \mid 2}\right)$ & 1.11 & - & 0.019 & - & 0.10 & 0.13 & 0.0 \\
\hline \hline
\end{tabular}

Tabela 6.21: Estimação parâmetros cópulas

Através dos valores estimados dos parâmetros, estamos aptos a determinar a função de distribuição $F_{X_{1}+X_{2}+X_{3}}$ como definido na Proposição 3 . O cálculo 
do valor do $\operatorname{VaR} R_{\alpha}\left(X_{1}+X_{2}+X_{3}\right)$ é obtido imediatamente a partir da função de distribuição da soma $F_{X_{1}+X_{2}+X_{3}}^{-1}(\alpha)=\operatorname{VaR}_{\alpha}\left(X_{1}+X_{2}+X_{3}\right)$. O gráfico da distribuição de $X_{1}+X_{2}+X_{3}$ é disposto a seguir.

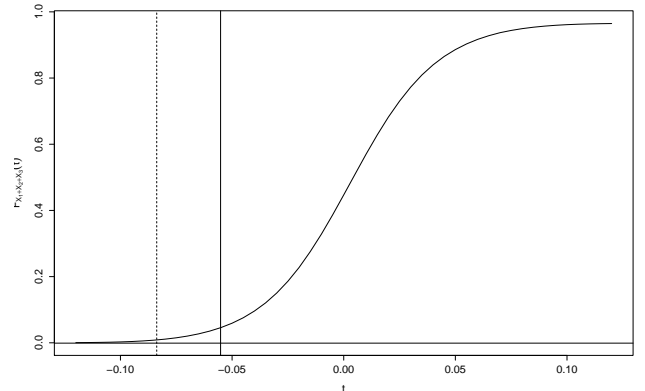

(a) $F_{X_{1}+X_{2}+X_{3}}(t)$

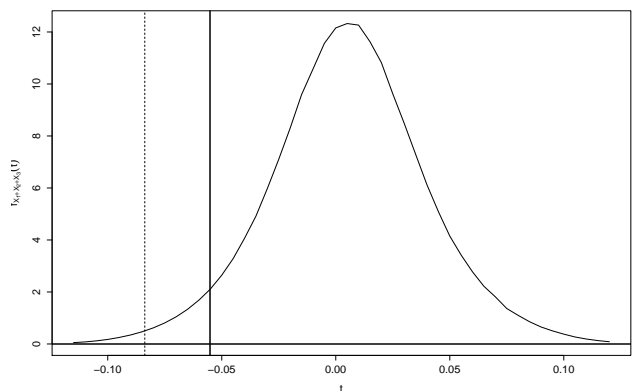

(b) $f_{X_{1}+X_{2}+X_{3}}(t)$

Figura 6.20: Value-at-Risk: $\operatorname{VaR}_{\alpha}\left(X_{1}+X_{2}+X_{3}\right) 1 \%$ (tracejada) $5 \%$ (contínua).

As medidas de risco VaR e ES da distribuição acima encontram-se na Tabela 6.22. Os valores foram arredondados até a quarta casa decimal.

\begin{tabular}{cccc}
\hline \hline Nível $\alpha$ & $V a R_{\alpha}\left(W_{3}\right)$ & $E S_{\alpha}\left(W_{3}\right)$ & $\sum_{i=1}^{3} V a R_{\alpha}\left(X_{i}\right)$ \\
\hline $5 \%$ & -0.0555 & -0.0649 & -0.0733 \\
$1 \%$ & -0.08375 & -0.0864 & -0.1313 \\
\hline \hline
\end{tabular}

Tabela 6.22: Medidas de risco de $W_{3}$.

Vamos considerar agora a característica heterocedástica dos retornos. A estrutura da distribuição conjunta dos retornos segue o mesmo Diagrama D-Vine 6.15. A modelagem da heterocedasticidade das marginais é feita por um processo AR(1)-GARCH(1,1), em que as inovações possuem distribuição t-Student. a Tabela 6.23 mostra as estimativas dos parâmetros das variâncias condicionais das marginais. 


\begin{tabular}{ccccccc}
\hline & \multicolumn{2}{c}{$X_{1}$} & \multicolumn{2}{c}{$X_{2}$} & \multicolumn{2}{c}{$X_{3}$} \\
\hline & $\hat{\Theta}$ & e.p. & $\hat{\Theta}$ & e.p. & $\hat{\Theta}$ & e.p. \\
\hline$\mu$ & $7.81 \cdot 10^{-4}$ & $3.13 \cdot 10^{-4}$ & $1.58 \cdot 10^{-3}$ & $3.23 \cdot 10^{-4}$ & $6.89 \cdot 10^{-4}$ & $1.8 \cdot 10^{-4}$ \\
$\phi$ & $-6.37 \cdot 10^{-3}$ & $1.76 \cdot 10^{-2}$ & $2.56 \cdot 10^{-3}$ & $3.02 \cdot 10^{-2}$ & $1.57 \cdot 10^{-1}$ & $1.57 \cdot 10^{-1}$ \\
$\omega$ & $6.69 \cdot 10^{-6}$ & $1.6 \cdot 10^{-4}$ & $1.32 \cdot 10^{-5}$ & $1.55 \cdot 10^{-4}$ & $3.84 \cdot 10^{-6}$ & $6.01 \cdot 10^{-5}$ \\
$\alpha$ & $6.6 \cdot 10^{-2}$ & $6.1 \cdot 10^{-2}$ & $9.51 \cdot 10^{-2}$ & $8.97 \cdot 10^{-2}$ & $1.3 \cdot 10^{-1}$ & $1.25 \cdot 10^{-1}$ \\
$\beta$ & $9.1 \cdot 10^{-1}$ & $4.35 \cdot 10^{-1}$ & $8.77 \cdot 10^{-1}$ & $3.15 \cdot 10^{-1}$ & $8.3 \cdot 10^{-1}$ & $3.94 \cdot 10^{-1}$ \\
\hline
\end{tabular}

Tabela 6.23: Estimação parâmetros marginais $X_{1}, X_{2}$ e $X_{3}$.

Em todos os casos observamos $\alpha+\beta<1$, isto é, os processos são estacionários. Já a Tabela 6.24 apresenta a estimativa dos parâmetros da D-Vine Cópula. Os valores foram arredondados até a segunda casa decimal.

\begin{tabular}{cccccccc}
\hline \hline Cópula & $\hat{\theta}$ & $\hat{\nu}$ & e.p $(\hat{\theta})$ & e.p $(\hat{\nu})$ & $\hat{\tau}$ & $\lambda_{U}$ & $\lambda_{L}$ \\
\hline$C_{12}\left(u_{1}, u_{2}\right)$ & 0.56 & 6.43 & 0.015 & 1.82 & 0.37 & 0.18 & 0.18 \\
$C_{23}\left(u_{2}, u_{3}\right)$ & 0.42 & 8.88 & 0.019 & 2 & 0.28 & 0.07 & 0.07 \\
$C_{13 \mid 2}\left(u_{1 \mid 2}, u_{3 \mid 2}\right)$ & 1.26 & - & 0.03 & - & 0.21 & 0.26 & 0.00 \\
\hline \hline
\end{tabular}

Tabela 6.24: Estimação parâmetros cópulas

A partir desses resultados estamos aptos a construir a curva cdf $F_{X_{1 t}+X_{2 t}+X_{3 t}}(r)$. Para cada tempo $t$ tem-se valores distintos para os parâmetros da cdf. Como explicitado anteriormente, o valor do $\operatorname{VaR}_{\alpha}\left(X_{1 t}+X_{2 t}+X_{3 t}\right)$ adquiri, como consequência, um comportamento dinâmico. A representação gráfica da evolução do VaR ao longo do tempo com $\alpha=0,05$, pode ser observada abaixo para o ano de 2015 , entre as datas $02 / 02 / 2015$ e $09 / 10 / 2015$. 

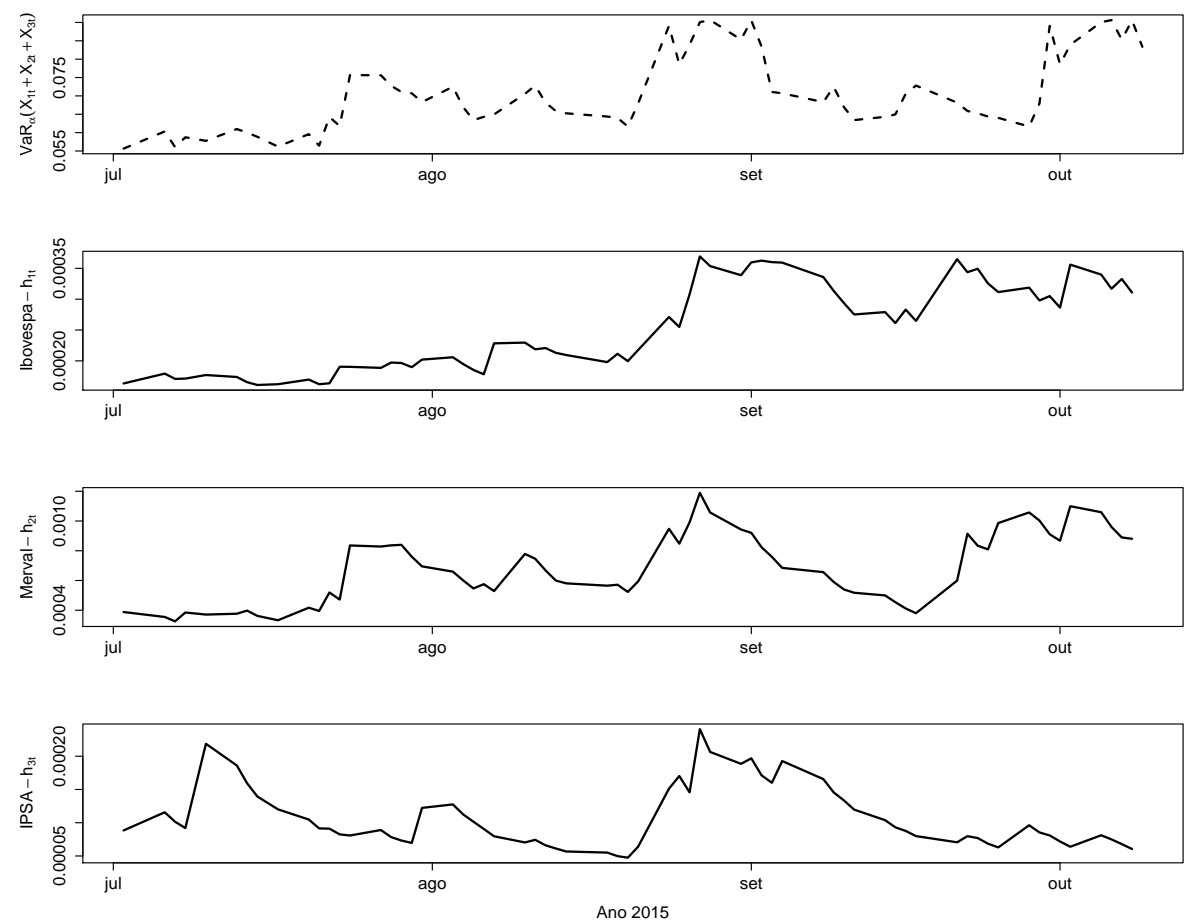

Figura 6.21: $\operatorname{VaR}_{\alpha}\left(X_{1 t}+X_{2 t}+X_{3 t}\right) \alpha=5 \%$ e variância condicionais dos índices

Observe que conforme a variância condicional dos retornos dos índices aumentam concomitante o valor do Value-at-Risk capta o acréscimo de risco e também aumenta, o que não ocorre no VaR estático. 



\section{Considerações Finais}

Neste trabalho tratamos da formulação de distribuição de funções de variáveis aleatórias dependentes do tipo soma $\sum_{i=1}^{n} X_{i}$, combinação convexa $\sum_{i=1}^{n} \pi_{i} X_{i}$, produto $X_{1} X_{2}$ e quocientes $X_{1} / X_{2}$ e $X_{1} /\left(X_{1}+X_{2}\right)$. A dependência entre as variáveis aleatórias foram induzidas via funções cópulas ao qual são incorporadas nas expressões destas distribuições. Os resultados do Capítulo 3 apresentam uma expressão alternativa ao método do Jacobiano para o desenvolvimento de distribuição destas funções. Do ponto de vista computacional, os resultados apontados oferecem um mecanismo geral para computar a distribuição da soma, combinação convexa, produto e quocientes de variáveis aleatórias.

O Capítulo 4 analisou o caso da distribuição da soma e combinação convexa em que as variáveis aleatórias são governadas por um processo $\mathrm{AR}(\mathrm{b})-\mathrm{GARCH}(\mathrm{p}, \mathrm{q})$ e as inovações possuem dependência. Uma contribuição em termos mais aplicados dos resultados exibidos neste capítulo é o cálculo do Value-at-Risk de forma dinâmica. Esta característica é de interesse para gestores de fundos e bancos para atualizar $\operatorname{VaR}_{\alpha, t}\left(W_{n t}\right)$ em cada tempo $t$.

Ao longo do desenvolvimento deste trabalho empregamos as R-Vines cópulas para construir uma distribuição multivariada. Entretanto, nenhuma expressão da distribuição das funções de variáveis aleatórias impôs sua utilização. Assim, as R-Vines foram aplicadas apenas como uma alternativa para a obtenção de cópulas multivariadas. Em virtude da construção de D-Vines ser por pares de cópulas e as distribuições condicionais dadas de forma recursiva, a obtenção da distribuição da soma e do valor $\operatorname{VaR}_{\alpha, t}\left(W_{n t}\right)$ tornam-se computacional intensiva. Esta dificuldade 
foi encontrada no Capítulo 6 na seção 6.2 onde empregamos cópulas trivariada por meio de D-Vines para computar o $\operatorname{VaR}_{\alpha, t}\left(W_{n t}\right)$ dos retornos dos principais índices das bolsas latino americanas, sendo este entrave proporcional ao valor de $n$. No que se refere à mistura de cópulas, nos limitamos apenas a apresentar a expressão da distribuição de funções de variáveis aleatórias. A restrição se deve ao método especial de estimação para misturas, além disso exige uma forma de seleção de cópulas para se ajustar aos dados também específica.

Os resultados apresentados neste trabalho restringiram-se a variáveis aleatórias contínuas. Em pesquisas futuras é possível estender os casos aqui apresentadas para as variáveis aleatórias discretas. Outra possibilidade é ampliar o estudo para diferentes funções de variáveis aleatórias comumente utilizadas na inferência estatística, como por exemplo, a razão de combinações lineares de variáveis, $\left(a_{1} X_{1}+a_{2} X_{2}\right) /\left(a_{3} X_{3}+a_{4} X_{4}\right)$ e $\sum_{i=1}^{Y} X_{i}$ para o caso discreto. No que tange a estudos empíricos, uma aplicação do $V_{a} R_{\alpha, t}\left(W_{n t}\right)$ dinâmico em alguns mercados e sua comparação com outros métodos também seria relevante para a literatura. O computo do $\operatorname{VaR}_{\alpha, t}\left(W_{n t}\right)$ dinâmico pode ainda incluir o caso onde o próprio parâmetro de dependência da cópula muda ao longo do tempo. Outra possibilidade é considerar o caso onde há mistura de funções cópulas na modelagem da dependência entre os dados do mercado financeiro. Do ponto de vista computacional, é possível avançar com o desenvolvimento de algoritmo e com a implementação de um pacote em softwares estatísticos para avaliar a distribuição de funções de variáveis aleatórias, incluindo as estudadas neste trabalho e demais casos. 


\section{Referências Bibliográficas}

Ali, M. M., Pal, M., e Woo, J. (2007). On the ratio of inverted gamma variates. Austrian Journal of Statistic, 36(2):153-159.

Arakelian, V. e Karlis, D. (2014). Clustering dependencies via mixture of copulas. Comunications in Statistics - Simulation and Computation, 43:1644-1661.

Artzner, P., Delbaen, F., Eber, J. M., e Heath, D. (1999). Coherent measure of risk. Mathematical Finance, 9:203-228.

Bedford, T. e Cooke, R. M. (2002). Vines - a new graphical model for dependent random variables. Annals of Statistics, 30:1031-1068.

Belgorodski, N. (2010). Selecting pair-copula families for regular vines with applications to multivariate analysis of european stock market indices. Dissertacao Mestrado - Universidade de München.

Brechmann, E. C. e Schepsmeier, U. (2013). Modeling dependence with c- and dvine copulas: The r package cdvine. Journal of Statistical Software, 52(3):1-27.

Breymann, W., Dias, A., e Embrechts, P. (2003). Dependence structure for multivariate high-frequency data in finance. Quantitative Finance, 3:1-14.

Cherubini, U., Luciano, E., e Vecchiato, W. (2004). Copula methods in finance. John Wiley \& Sons Ltd.

Cherubini, U., Mulinacci, S., e Romagnoli, S. (2011). On the distribution of the (un)bounded sum of random variables. Insurance: Mathematics and Economics, 48:56-63. 
Choudhry, M. (2006). An Introduction to Value at Risk. John Wiley.

Cont, R. (2001). Empirical properties of asset returns: stylized facts and statistical issues. Quantitative Finance, 1:223-236.

Durbin, J. e Stuart, A. S. (1951). Inversions and rank correlations. Journal of Royal Statistical Society Series, 2:303-309.

Embrechts, P. Hoing, A. e Juri, A. (2003). Using copulae to bound the value-at-risk for functions of dependent risks. Finance and Stochastics, 7:145-167.

Embrechts, P., Lindskog, F., e McNeil, A. (2001a). Modelling Dependence with Copulas and Applications to Risk Management. Zurich, Swiss.

Embrechts, P., McNeil, A., e Straumann, D. (2001b). Correlation and dependency in risk management:properties and pitfalls. In: Dempster, M. et al. (Eds.), Risk Management: Value at Risk and Beyond. Cambridge University Press, Cambridge.

Fisher, R. A. e Tippett, L. H. C. (1928). Limiting forms of frequency distributions of the largest or smallest member of a sample. Philosophical Society, 24:180-190.

Genest, C., , e Rivest, L. P. (1993). Statistical inference procedures for bivariate archimedean copulas. Journal of the American Statistical Association, 88(423):1034-1043.

Genest, C. e Favre, A. C. (2007). Everything you always wanted to know about copula modeling but were afraid to ask. Journal of Hydrologic Engineering, $12: 347-368$.

Genest, C. e MacKay, J. (1986). The joy of copulas: Bivariate distributions uniform marginals. The American Statistician, 40:280-283.

Haff, I. H., Aas, K., e Frigessi, A. (2010). On the simplified pair-copula constrution - simply useful or simplistic? Journal of Multivariate Analysis, 101(5):1296 1310. 
Hall, P., Horowitz, J. L., e Jing, B. (1995). On blocking rules for bootstrap with dependent data. Biometrika, 82:561-574.

Hofmann, M. e Czado, C. (2011). Assessing the var of portfolio using d-vine copula based multivariate garch models. Working Paper -

Idrizi, L. (2014). On the product and ratio of pareto and kumaraswamy random variable. Mahtematical Theory and Modeling, 4(3):136-146.

Irene, G. e Klaus, H. (2014). On the distribution of sums of random variables with copula-induced dependence. Insurance: Mathematics and Economics, 59:27 44.

James, B. R. (2011). Probabilidade: um curso em nivel intermediário. IMPA.

Jenkinson, A. F. (1955). The frequency distribution of annual maximum (or minimum) values of meteorological elements. Quartely Journal of the Royal Meteorology Society, 87:145-158.

Joe, H. (1996). Families of m-variate distributions with given margins and m(m1)/2 bivariate dependence parameters. Distributions with Fixed Marginals and Related Topics, 28:120-141.

Jondeau, E., Poon, S., e Rockinger, M. (2007). Financial Modeling Under NonGaussian Distribution. Springer.

Libby, D. L. e Novick, M. R. (1982). Multivariate generalized beta distributions with applications to utility assessment. Journal of Educational and Behavioral statistic, 4:271-294.

Nadarajah, S. (2005a). Sums, product and ratios for the bivariate gumbel distribution. Mathematical and Computer Modelling, 42:499-518.

Nadarajah, S. (2005b). Sums, product and ratios for the bivariate lomax distribution. Computational Statistics \& Data Analysis, 49:109-129. 
Nadarajah, S. e Ali, M. M. (2006). The distribution of sums, product and ratios for lawrance and lewis's bivariate exponential random variables. Computational Statistics \& Data Analysis, 50:3449-3463.

Nadarajah, S. e Dey, D. K. (2006). On the product and ratio of t random variables. Applied Mathematics Letters, 19:45-55.

Nagar, D. A., Orozco-Castaneda, J. M., e Gupta, A. K. (2009). Product and quotient of correlated beta variables. Applied Mathematics Letters, 22:105-109.

Nelsen, R. B. (2006). An Introduction to Copulas. Springer.

Patton, A. (2001). Modelling time-varying exchange rate dependence using the conditional copula. Working Paper - UC San Diego Department of Economics.

Pham-Gia, T. (2000). Distributions of the ratios of independent beta variables and aplications. Communications in Statistics - Theory and Methods, 29(12):26932715 .

Pickands, J. (1981). Multivariate extreme value distributions. Bull. Int. Statist. Inst, pages 859-878.

Press, S. J. (1969). The t-ratio distribution. Journal of the American Statistical Association, 64(325):242-252.

R Core Team (2014). R: A Language and Environment for Statistical Computing. R Foundation for Statistical Computing, Vienna, Austria.

Shakil, M. abd Golam Kibria, B. M. (2006). Exact distribution of the ratio of gamma and rayleigh random variables. Pakistan Journal of Statistics and Operation Research, 2(2):87-98.

Sklar, A. (1973). Random variables, joint distribution functions, and copulas. Kybernetica, 9:449-460. 
Song, P. X., Fan, K. Y., e Kalbfleisch, J. D. (2005). Maximization by parts in likelihood inference. Journal of the American Statistical Association, 100:11451158.

Trivedi, P. K. e Zimmer, D. M. (2005). Copulas Modeling: An Introduction for Practitioners. Foundations and Trends in Econometrics.

Vuong, Q. H. (1989). Likelihood ratio tests for model selection and non-nested hypotheses. Econometrica, 57(2):307-333. 


\section{Apêndice A}

Teorema 12 (Prova por Indução Matemática) Seja $P(n)$ uma sentença aberta sobre $\mathbb{N}$. Suponha que,

i) $\mathrm{P}(1)$ é verdadeira; e,

ii) qualquer que seja $n \in \mathbb{N}$, sempre que $P(n)$ é verdadeira, segue que $P(n+1)$ é verdadeira

Então, $P(n)$ é verdadeira para todo $n \in \mathbb{N}$.

Teorema 13 (Distribuição da Função Acumulada) Seja $X$ uma variável aleatória contínua com distribuição acumulada $F_{X}(x)$ e seja também a variável a aleatória $Y=F_{X}(X)$, então $Y$ tem distribuição uniforme, i.e. $Y \sim U(0,1)$. Portanto, $F_{Y}(y)=P(Y \leq y)=y$ e $f_{Y}(y)=1$, para $0 \leq y \leq 1$.

Prova. A transformação $Y=F_{X}(X)$ está definida para $0 \leq y \leq 1$. Além disso, tem-se que,

$$
\begin{aligned}
F_{Y}(y) & =P(Y \leq y)=P\left(F_{X}(X) \leq y\right) \\
& =P\left(F_{X}^{-1}\left(F_{X}(X)\right) \leq F_{X}^{-1}(y)\right) \\
& =P\left(X \leq F_{X}^{-1}(y)\right) \\
& =F_{X}\left(F_{X}^{-1}(y)\right) \\
& =y
\end{aligned}
$$


Derivando $F_{Y}(y)$ com relação a $y$

$$
\frac{d}{d y} F_{Y}(y)=\frac{d}{d y} y=1
$$

Logo, como temos que $0 \leq y \leq 1$, então a distribuição de $Y \sim U(0,1)$.

Proposição 11 Seja $X$ uma variável aleatória com distribuição t-Student padronizada, $X \sim t(\nu)$ em que $\nu>0$ são os graus de liberdade, então a distribuição acumulada da distribuição t-Student pode ser escrita por,

$$
F(x ; \nu)=\frac{1}{2}+\frac{x \Gamma\left(\frac{\nu+1}{2}\right)}{\sqrt{\pi \nu} \Gamma\left(\frac{\nu}{2}\right)}{ }_{2} F_{1}\left(\frac{1}{2}, \frac{\nu+1}{2} ; \frac{3}{2} ;-\frac{x^{2}}{\nu}\right) .
$$

Prova. Tomando ${ }_{2} F_{1}$ a função hipergeométrica (Série Hipergeométrica de Gauss) que pode ser expressa pela série de potências,

$$
{ }_{2} F_{1}(a, b ; c ; x)=\sum_{n=0}^{\infty} \frac{(a)_{n}(b)_{n}}{(c)_{n}} \frac{x^{n}}{n !}
$$

onde converge para $|x|<1$ e $(\xi)_{n}$ representa o símbolo de Pochhammer, o qual é definido por,

$$
\begin{aligned}
(\xi)_{n} & \equiv \prod_{k=0}^{n-1}(\xi+k)=(\xi)(\xi+1)(\xi+2) \cdots(\xi+n-1), \\
& =\frac{\Gamma(\xi+n)}{\Gamma(\xi)}=\left(\begin{array}{c}
-\xi \\
n
\end{array}\right) n !(-1)^{n} .
\end{aligned}
$$

Utilizando esta função e o fato da densidade ser uma função par, então tomamos a seguinte integral,

$$
\begin{aligned}
F(x ; \nu) & =\int_{-\infty}^{x} f(t ; \nu) d t \\
& =\frac{1}{2}+\int_{0}^{x} \frac{\Gamma\left(\frac{\nu+1}{2}\right)}{\Gamma\left(\frac{\nu}{2}\right) \sqrt{\pi \nu}}\left(1+\frac{t^{2}}{\nu}\right)^{-\frac{\nu+1}{2}} d t
\end{aligned}
$$


Aplicando a expansão binomial.

$$
\begin{aligned}
& F(x ; \nu)=\frac{1}{2}+\frac{\Gamma\left(\frac{\nu+1}{2}\right)}{\Gamma\left(\frac{\nu}{2}\right) \sqrt{\pi \nu}} \int_{0}^{x} \sum_{j=0}^{\infty}\left(\begin{array}{c}
-\frac{\nu+1}{2} \\
j
\end{array}\right)\left[\frac{t^{2}}{\nu}\right]^{j} d t \\
& =\frac{1}{2}+\frac{\Gamma\left(\frac{\nu+1}{2}\right)}{\Gamma\left(\frac{\nu}{2}\right) \sqrt{\pi \nu}} \sum_{j=0}^{\infty}\left(\begin{array}{c}
-\frac{\nu+1}{2} \\
j
\end{array}\right) \frac{1}{\nu^{j}} \int_{0}^{x} t^{2 j} d t \\
& =\frac{1}{2}+\left.\frac{\Gamma\left(\frac{\nu+1}{2}\right)}{\Gamma\left(\frac{\nu}{2}\right) \sqrt{\pi \nu}} \sum_{j=0}^{\infty}\left(\begin{array}{c}
-\frac{\nu+1}{2} \\
j
\end{array}\right) \frac{1}{\nu^{j}} \frac{t^{2 j+1}}{2 j+1}\right|_{0} ^{x} \\
& =\frac{1}{2}+\frac{\Gamma\left(\frac{\nu+1}{2}\right)}{\Gamma\left(\frac{\nu}{2}\right) \sqrt{\pi \nu}} \sum_{j=0}^{\infty}\left(\begin{array}{c}
-\frac{\nu+1}{2} \\
j
\end{array}\right) \frac{1}{\nu^{j}} \frac{x^{2 j+1}}{2 j+1}
\end{aligned}
$$

Utilizando o símbolo de Pochhammer temos que,

$$
\begin{aligned}
F(x ; \nu) & =\frac{1}{2}+\frac{x \Gamma\left(\frac{\nu+1}{2}\right)}{\Gamma\left(\frac{\nu}{2}\right) \sqrt{\pi \nu}} \sum_{j=0}^{\infty} \frac{1}{j !}(-1)^{j}\left(\frac{\nu+1}{2}\right)_{j} \frac{1}{2 j+1}\left(x^{2} / \nu\right)^{j} x \\
& =\frac{1}{2}+\frac{x \Gamma\left(\frac{\nu+1}{2}\right)}{\Gamma\left(\frac{\nu}{2}\right) \sqrt{\pi \nu}} \sum_{j=0}^{\infty} \frac{1}{j !}(-1)^{j}\left(\frac{\nu+1}{2}\right)_{j} \frac{(1 / 2)_{j}}{(3 / 2)_{j}}\left(x^{2} / \nu\right)^{j} \\
& =\frac{1}{2}+\frac{x \Gamma\left(\frac{\nu+1}{2}\right)}{\Gamma\left(\frac{\nu}{2}\right) \sqrt{\pi \nu}} \sum_{j=0}^{\infty}\left(\frac{\nu+1}{2}\right)_{j} \frac{(1 / 2)_{j}}{(3 / 2)_{j}} \frac{\left(-x^{2} / \nu\right)^{j}}{j !} \\
& =\frac{1}{2}+\frac{x \Gamma\left(\frac{\nu+1}{2}\right)}{\Gamma\left(\frac{\nu}{2}\right) \sqrt{\pi \nu}}{ }_{2} F_{1}\left(\frac{1}{2}, \frac{\nu+1}{2} ; \frac{3}{2} ;-\frac{x^{2}}{\nu}\right),
\end{aligned}
$$

onde converge para $x^{2}<\nu$.

Proposição 12 Sejam as variáveis aleatórias $X_{1} \sim \Gamma(\alpha, \lambda) X_{2} \sim \Gamma(\beta, \lambda)$ e sendo $\alpha$ e $\beta$ parâmetros de forma e $\lambda$ de escala, então a distribuição da razão $Y=\frac{X_{1}}{X_{1}+X_{2}}$ tem distribuição beta. Equivalentemente escrevemos $Y \sim B(\alpha, \beta)$, onde possui a seguinte função densidade,

$$
f_{Y}(y)=\frac{1}{B(\alpha, \beta)} y^{\alpha-1}(1-y)^{\beta-1} \text { em que } 0 \leq y \leq 1
$$

Onde $B(\alpha, \beta)=\frac{\Gamma(\alpha) \Gamma(\beta)}{\Gamma(\alpha+\beta)}$ é a constante normalizadora.

Prova. A caracterização da distribuição resultante da razão é direta. Sendo $X_{1}$ e 
$X_{2}$ independentes, então a distribuição conjunta pode ser escrita por,

$$
f_{X_{1}, X_{2}}\left(x_{1}, x_{2}\right)=\frac{1}{\Gamma(\alpha) \lambda^{\alpha}} x_{1}^{\alpha-1} e^{-x_{1} / \lambda} \frac{1}{\Gamma(\beta) \lambda^{\beta}} x_{2}^{\beta-1} e^{-x_{2} / \lambda}
$$

Aplicando as transformações,

$$
Y=\frac{X_{1}}{X_{1}+X_{2}}, \quad W=X_{1}
$$

O jacobinano $J$ desta transformação fica,

$$
J=\left|\begin{array}{ll}
\frac{\partial x_{1}}{\partial y} & \frac{\partial x_{1}}{\partial w} \\
\frac{\partial x_{2}}{\partial y} & \frac{\partial x_{2}}{\partial w}
\end{array}\right|=\frac{w}{y^{2}}
$$

Assim, a função densidade de $Y$ e $W$ fica,

$$
\begin{aligned}
f_{W, Y}(w, y) & =f_{X_{1}, X_{2}}\left(w, \frac{1-y}{y} w\right)|J| \\
& =\frac{1}{\Gamma(\alpha) \lambda^{\alpha}} w_{1}^{\alpha-1} e^{-w_{1} / \lambda} \frac{1}{\Gamma(\beta) \lambda^{\beta}}\left(\frac{1-y}{y} w\right)^{\beta-1} e^{-\frac{1-y}{y \lambda} w} \frac{w}{y^{2}} .
\end{aligned}
$$

A distribuição marginal de $Y$ é dada por,

$$
\begin{aligned}
f_{Y}(y) & =\int_{0}^{\infty} \frac{1}{\Gamma(\alpha) \lambda^{\alpha} \Gamma(\beta) \lambda^{\beta}}\left(\frac{1-y}{y}\right)^{\beta-1} \frac{1}{y^{2}} w^{\alpha+\beta-1} e^{-w / \lambda y} d w, \\
& =\frac{1}{\Gamma(\alpha) \lambda^{\alpha} \Gamma(\beta) \lambda^{\beta}}\left(\frac{1-y}{y}\right)^{\beta-1} \frac{1}{y^{2}} \int_{0}^{\infty} w^{\alpha+\beta-1} e^{-w / \lambda y} d w, \\
& =\frac{1}{\Gamma(\alpha) \Gamma(\beta) \lambda^{\alpha+\beta}}(1-y)^{\beta-1} y^{-(\beta-1)-2} \Gamma(\alpha+\beta) y^{\alpha+\beta} \lambda^{\alpha+\beta}, \\
& =\frac{\Gamma(\alpha+\beta)}{\Gamma(\alpha) \Gamma(\beta)}(1-y)^{\beta-1} y^{\alpha-1}, \text { em que } 0 \leq y \leq 1,
\end{aligned}
$$

o qual é igual a (2).

Definição 15 (Value-at-Risk) Dado $\alpha \in(0,1)$ o Value-at-Risk $\operatorname{VaR}_{\alpha}(X)$ ao nível $\alpha$ de um portfólio com valor final ou retorno $X$ com probabilidade $P$ é o 
quantil $q_{\alpha}$ de $X$, tal que,

$$
\operatorname{VaR}_{\alpha}(X)=-\sup \{x \in \mathbb{R} \mid P(X \leq x) \leq \alpha\}
$$

Definição 16 (Expected Shortfalls) Seja $X$ a variável aleatória retorno do portfólio e I uma variável indicadora, o Expected Shortfalls - ES é expresso por,

$$
E S_{\alpha}(X)=-\frac{1}{\alpha}\left(\mathbb{E}\left[X \boldsymbol{I}_{\left\{X \leq x_{\alpha}\right\}}\right]+x_{\alpha}\left(\alpha-P\left[X \leq x_{\alpha}\right]\right)\right)
$$

em que, $x_{\alpha}=\sup \{x \in \mathbb{R}: P(X \leq x) \leq \alpha\}$. No caso em que a distribuição de $X$ é contínua, então o ES é equivalente a,

$$
E S_{\alpha}(X)=-\mathbb{E}\left[X \mid X \leq-\operatorname{VaR}_{\alpha}(X)\right]
$$

Definição 17 (Índice de Sharpe) O Índice de Sharpe é calculado por,

$$
S R_{i}=\frac{\mathbb{E}\left[X_{i}-R_{f}\right]}{\sigma_{i}}
$$

em que $X_{i}$ é a taxa de retorno do ativo $i, R_{f}$ a taxa de retorno livre de risco.

Artzner et al. (1999) definem quatro condições para que uma medida de risco seja dita coerente.

Definição 18 Seja $\mathcal{G}$ um conjunto de variáveis aleatórias. A função $\rho: \mathcal{G} \rightarrow \mathbb{R}$ é uma medida de risco coerente se satisfaz:

1. Invariância por Translação Para $X \in \mathcal{G}$ e $\gamma \in \mathbb{R}$ temos $\rho(X+\gamma)=$ $\rho(X)-\gamma$.

2. Subaditividade: Se $X_{1}$ e $X_{2} \in \mathcal{G}$ então $\rho\left(X_{1}+X_{2}\right) \leq \rho\left(X_{1}\right)+\rho\left(X_{2}\right)$.

3. Homogeneidade Positiva: Se $\lambda \geq 0$ e $X \in \mathcal{G}$ então $\rho(\lambda X)=\lambda \rho(X)$, 
4. Monotonicidade: Para $X_{1}$ e $X_{2} \in \mathcal{G}$ onde $X_{1} \leq X_{2}$ então $\rho(Y) \leq \rho(X)$. 


\section{Apêndice B}

\begin{tabular}{cccc}
\hline \hline Índice & DF Teste & Lag Ordem & p-Valor \\
\hline Ibovespa & -13.6459 & 14 & $0.01^{*}$ \\
Merval & -13.8645 & 14 & $0.01^{*}$ \\
IPSA & -12.9821 & 14 & $0.01^{*}$ \\
\hline \hline
\end{tabular}

Tabela 25: Teste estacionaridade ADF dos retornos

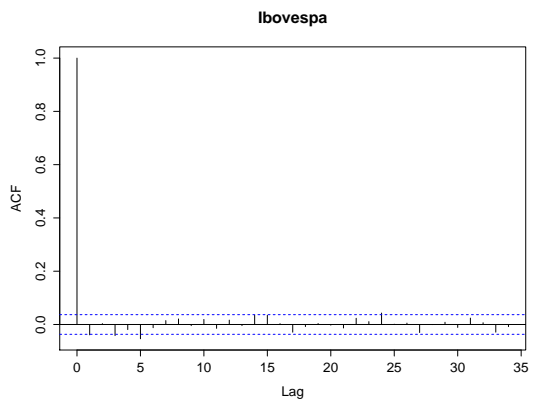

(a) Ibovespa

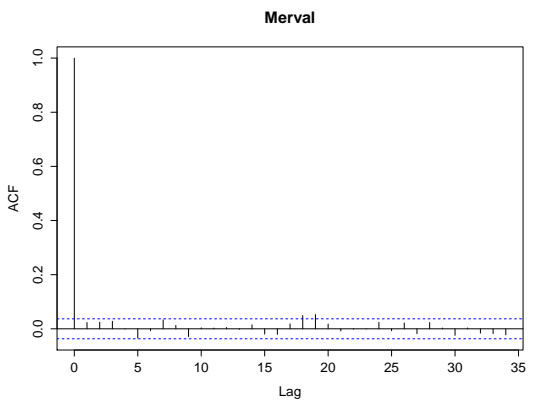

(b) Merval

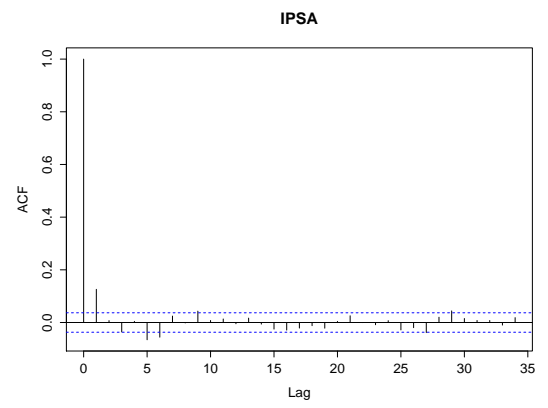

(c) IPSA

Figura 22: Função autocorrelação dos retornos

\begin{tabular}{cccc}
\hline \hline Índice & $\chi^{2}$ & g.l & p-Valor \\
\hline Ibovespa & 4.2986 & 1 & 0.03814 \\
Merval & 1.5256 & 1 & 0.2168 \\
IPSA & 44.8769 & 1 & $0.01^{*}$ \\
\hline \hline
\end{tabular}

Tabela 26: Teste de autocorrelação Ljung-Box retornos 


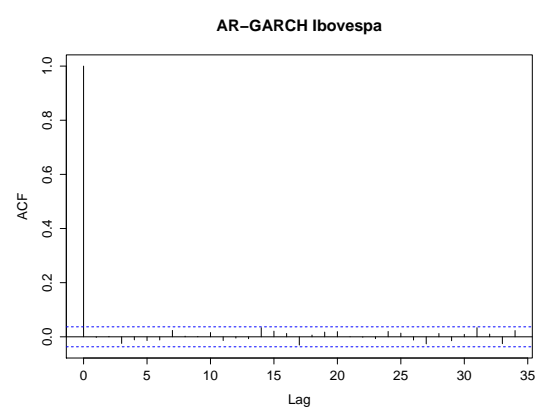

(a) Ibovespa

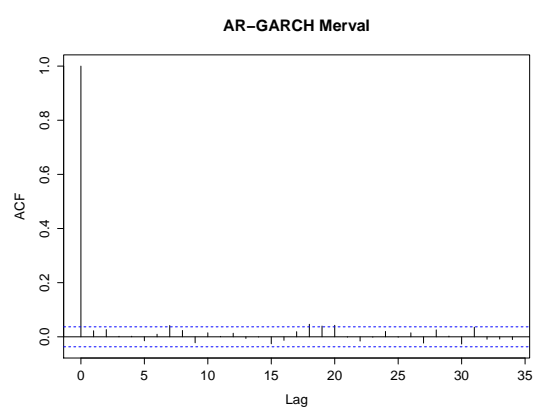

(b) Merval

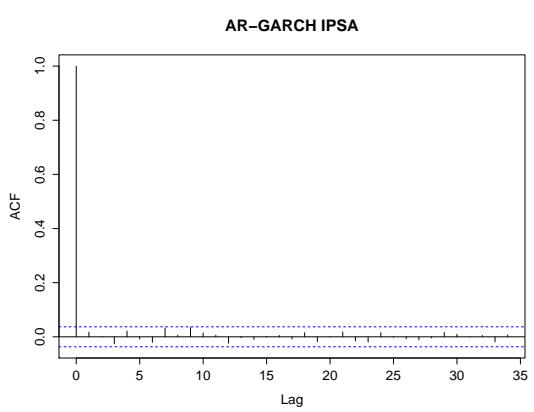

(c) IPSA

Figura 23: Função autocorrelação dos resíduos AR(1)-GARCH(1,1)

\begin{tabular}{cccc}
\hline \hline Índice & $\chi^{2}$ & g.l & p-Valor \\
\hline Ibovespa & 0.0162 & 1 & 0.8987 \\
Merval & 1.4148 & 1 & 0.2343 \\
IPSA & 0.8657 & 1 & 0.3521 \\
\hline \hline
\end{tabular}

Tabela 27: Teste de autocorrelação Ljung-Box resíduos $\mathrm{AR}(1)-\mathrm{GARCH}(1,1)$ 